Universidade de São Paulo

Tatiana Ferreira de Almeida

Diagnóstico molecular do transtorno do espectro autista através do sequenciamento completo de exoma

\title{
Molecular diagnosis of autism spectrum disorder through whole exome sequencing
}


Universidade de São Paulo

Tatiana Ferreira de Almeida

\section{Diagnóstico molecular do transtorno do espectro autista através do sequenciamento completo de exoma}

\section{Molecular diagnosis of autism spectrum disorder through whole exome sequencing}

Tese apresentada ao Instituto de Biociências da Universidade de São Paulo, para a obtenção de Título de Doutor em Ciências, na Área de Biologia/Genética. Versão corrigida.

Orientador(a): Maria Rita dos Santos Passos-Bueno 
Dedico esta tese à ciência, e a todos os seus amantes.

$O$, reason not the need! Our basest beggars Are in the poorest thing superfluous. Allow not nature more than nature needs, Man's life is cheap as beast's. Thou art a lady: If only to go warm were gorgeous, Why, nature needs not what thou gorgeous wear'st Which scarcely keeps thee warm. But, for true needYou heavens, give me that patience, patience I need! You see me here, you gods, a poor old man, As full of grief as age; wretched in both." 


\section{Agradecimentos}

Agradeço em primeiro lugar ao meu grande amor Danilo, sem ele, há 11 anos, essa jornada não teria começado, a vida não teria me surpreendido e eu estaria de volta em casa, com todos os peixinhos do mar, e infeliz pelo resto da minha vida.

Agradeço minha família, principalmente minha mãe Flávia e meu pai Airton por sacrificarem com mínimos protestos, as melhores horas que passaríamos juntos para que eu cumprisse meu trabalho. Aos compadres e amigos Bruna e Wagniel e Cíntia e Fernando que não somente me apoiaram neste caminho, mas colocaram na minha vida os maiores presentes que eu poderia sonhar, Alice e Juliana, que mesmo em tão pouco tempo de vida e com tantas ausências ainda sorriem ao me ver.

À minha orientadora Maria Rita Santos Passos-Bueno que com um raciocínio feroz e implacável não mediu esforços para me ajudar a percorrer o caminho mais desafiador que eu já enfrentei. Ao professor Paulo Otto que solidificou meu amor pela matemática e me ajudou a pensar em probabilidades.

Agradeço ao Renato Puga que pegou na minha mão e me ensinou a programar (em awk!!), obrigada por ser o ursinho toda vez que eu errava uma vírgula. Jovem, "Até logo, e obrigada pelos pães!"

Aos amigos de laboratório, o Lab200, que me ensinaram a força da cooperação, a pensar em todos. Eu nunca tinha visto pessoas trabalharem com tamanha vontade de fazer o melhor por todos e espero ter aprendido com vocês e levar para a vida essa tão valiosa lição. Gerson, Lucas e Luciano obrigada pela constante inspiração. Obrigada meninas do GeneEvol, Carol, Ágatha, Camila e Clarice, e mais tarde Duda, por me deixarem entrar na vida de vocês e sempre lembrarem de me incluir nos encontros mesmo eu quase nunca podendo ir.

Aos amigos do DLE, principalmente Dayse, Karina, Cíntia e Mi, que sempre me viam correndo de um lado pro outro com as "coisas do doutorado" e foram compreensivos e pacientes com a vida dupla que eu levava. Ao Gustavo Campana que me mostrou como deve ser um exame para o diagnóstico laboratorial e me encantou com esse mundo da patologia clínica.

Às amadas bailarinas, obrigada por fazerem da minha vida melhor e mais divertida, nada disso teria sido possível se não fosse minha terapia em roupas de ballet. Lili, obrigada por me ensinar tanto na vida e no palco. Paty, a bola é sua agora!!

E finalmente à CAPES pelo apoio financeiro durante o percurso deste trabalho. 


\section{Table of Contents}

1 Introduction

1.1 Clinical and epidemiological aspects of autism spectrum disorder ................1

1.2 Molecular aspects of autism spectrum disorder .................................................. 3

1.3 Next Generation Sequencing and the Diagnosis of Rare Diseases .................. 6

1.4 Next Generation Sequencing and the ASD Diagnosis ....................................... 8

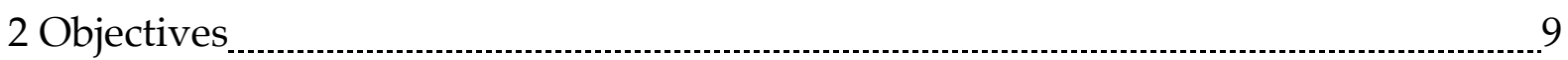

3 Material and Methods

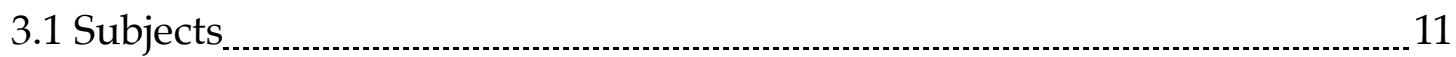

3.1.1 Austism Spectrum Disorder Subjects _............................................11

3.1.2 Control group

3.1.3 Kinship and ancestry analysis _..................................................... 15

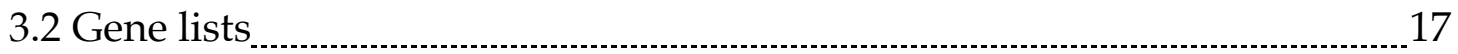

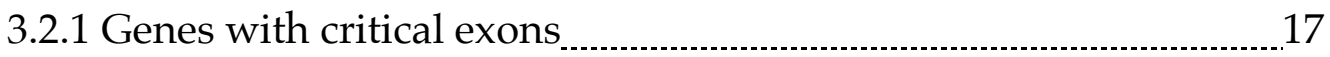

3.2.2 Chromatin modifier genes ………..................................................... 18

3.2.3 1527 genes related to ASD ............................................................ 18

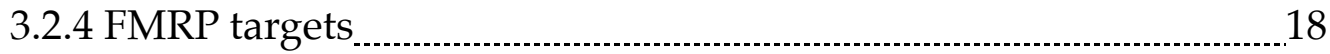

3.2.5 208 genes related to ASD ....................................................................... 19

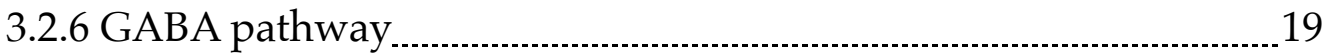

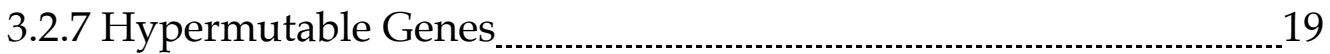

3.2.8 mTOR and RAS/MAPK Pathways _............................................... 20

3.2.9 IGF1 Pathway _..._. 20

3.2.10 Genes intolerant to loss-of-function mutations _............................ 20

3.2.11 Differentially expressed genes _.................................................21 


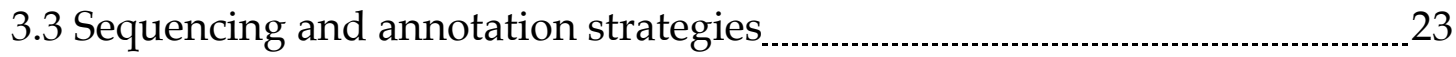

3.4 Comparisons pipeline

3.4.1 Rare Loss-of-function and missense

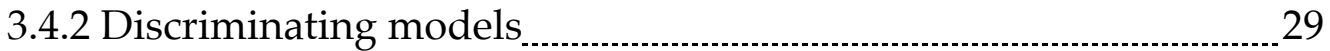

3.4.2.1 Classification Performance ……........................................... 29

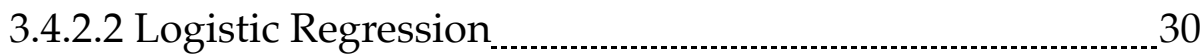

3.4.2.3 Decision Tree

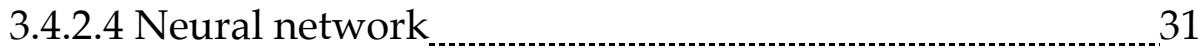

3.4.2.5 Support Vector Machine

3.4.3 Principal Component Analysis ........................................................ 32

4 Results

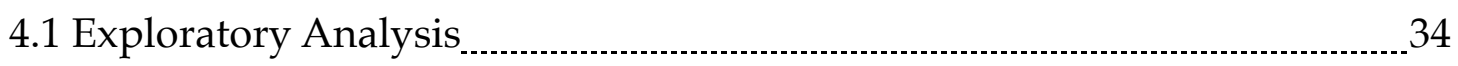

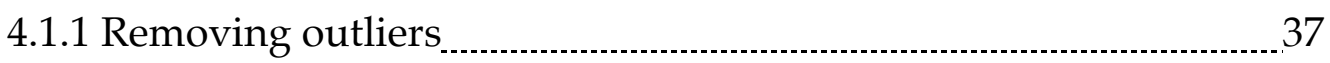

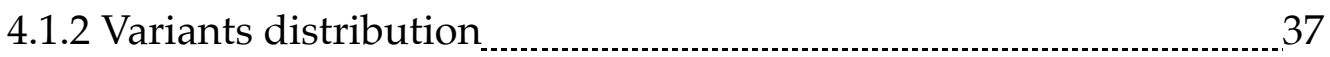

4.1.3 Kmeans

4.1.4 Correlation Matrices

4.1.5 Variant distribution between affected and control .......................... 42

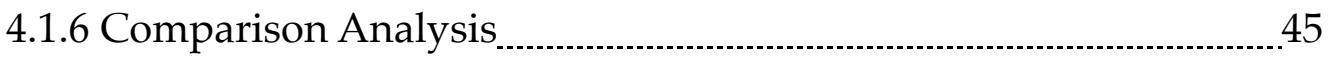

4.1.6.1 Loss-of-Function grouping ................................................ 45

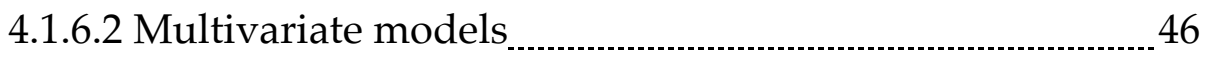

4.1.6.3 Principal Components Analysis ........................................... 47

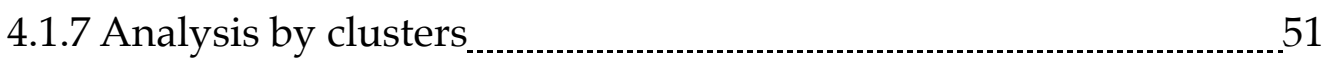

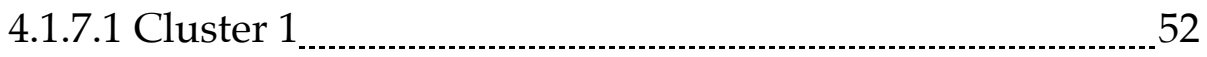

4.1.7.2 Cluster 2

4.1.8 Separate regions 
4.2.1 Genes with critical exons 55

4.2.2 Chromatin modifier genes 56

4.2.3 1,527 genes related to ASD 57

4.2.4 FMRP targets 57

4.2.5 208 genes related to ASD 59

4.2.6 GABA pathway. 59

4.2.7 Hipermutable Genes 60

4.2.8 mTOR Pathway 60

4.2.9 IGF1 Pathway. 61

4.2.10 Genes intolerant to loss-of-function mutations 61

4.2.11 Differentially expressed genes M1 62

4.2.12 Differentially expressed genes M2 62

4.2.13 Differentially expressed genes M8 63

4.2.14 Differentially expressed genes M16 63

4.2.15 Differentially expressed genes M18 65

4.2.16 RAS/MAPK Pathway 66

4.2.17 SFARI High Confidence 68

4.2.18 SFARI Strong Candidate 68

4.2.19 SFARI Suggestive Evidence 68

4.2.20 SFARI Syndromic..... 68

4.2.21 SFARI High Confidence, Strong Evidence, Suggestive Evidence and Syndromic... 69

5 Discussion and Conclusions 69

6 Resumo 77

7 Abstract 79

8 Reference 81 
?? Attachments

?? Attachment 1

?? Attachment 2

?? Attachment 3

?? Attachment 4

?? Attachment 5

?? Attachment 6

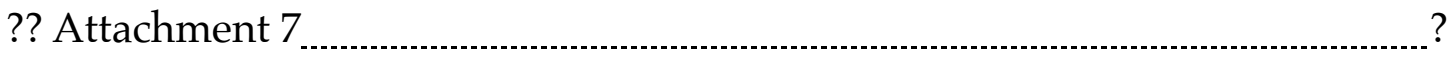

?? Attachment 8

?? Attachment 9

?? Attachment 10

?? Attachment 11

?? Attachment 12

?? Attachment 13

?? Attachment 14

?? Attachment 15

?? Attachment 16

?? Attachment 17

?? Attachment 18

?? Attachment 19

?? Attachment 20

?? Attachment 21

?? Attachment 22

?? Prefilter

?? Spring

?? Counting 
?? Attachment 23

?? Attachment 24 ?

?? Attachment 25 ? 


\section{Introduction}

\subsection{Clinical and epidemiological aspects of autism spectrum disorder}

Autism spectrum disorders (ASD) are a group of neurodevelopmental disorders characterized by impaired communication skills, behavior and social interaction. According to the DSM-5 criteria (Diagnostic and Statistical Manual of Mental Disorders, 5th edition), the diagnosis of ASD is made based on clinical findings in two different categories, namely: (1) impaired social and communication skills and (2) stereotypic behavior and repetitive motions (LA TORRE-UBIETA et al., 2016; LORD; BISHOP, 2015). Social-communicative skill changes must present deficits in three distinct areas: (a) socio-emotional reciprocity, (b) nonverbal behaviors used in social interaction, and (c) maintaining, developing and understanding relationships, in addition to adjusting to various social contexts (LORD; BISHOP, 2015). This new classification encompasses every pervasive developmental disorder previously sorted into Asperger's syndrome, autism, childhood disintegrative disorders, and unspecified pervasive developmental disorders. According to the new classification criteria, although there is no age limit for symptom onset, all the individuals with ASD should present them from childhood, even if they are only recognized later.

In addition to these core characteristics, ASD can present highly complex clinical features and harbor many comorbidities, where $50 \%$ of the cases present language delay, $35-50 \%$ are associated with intellectual disability (ID), approximately $21.5 \%$ of individuals with ASD and ID are affected by epilepsy, while only $8 \%$ of them present average intellect (BOURGERON, 2015; GESCHWIND; STATE, 2015; WOODBURY-SMITH; SCHERER, 2018). Gastrointestinal problems, sleep disorders and psychiatric disorders are also common ASD comorbidities (ANAGNOSTOU et al., 2014).

Approximately $10-25 \%$ of the ASD cases are part of a recognizable Mendelian disorder, such as fragile X-Syndrome (1.9\%), Rett syndrome, tuberous sclerosis $(0.9 \%)$, neurofibromatosis type $1(0.28 \%)$, Williams-Beuren syndrome and many others (ANAGNOSTOU et al., 2014; LA TORRE-UBIETA et al., 2016), a great number with a known molecular basis that can be tested on suspected cases. Thus, routine screening for Rett syndrome and fragile $X$ syndrome should be performed on individuals with ASD (SCHAEFER; MENDELSOHN, 2013). This particular characteristic confers ASD the label of being a common syndrome that, at least in part, comprises several rare disorders (GESCHWIND; STATE, 2015).

The available data suggest that at least some forms of ASD involve time-specific developmental deficits that might be targets for treatment, even after the symptoms first emerge (BETANCUR; SAKURAI; BUXBAUM, 2009). Many drugs are being tested, based on pathways and specific gene targets (GRIESI-OLIVEIRA et al., 2014; 
LIN et al., 2014; RIIKONEN, 2016), but the only FDA-approved drugs, risperidone and aripiprazole, are not oriented to the core social deficits (LA TORRE-UBIETA et al., 2016).

According to a meta-analysis conducted in 2014, the ASD prevalence worldwide was estimated at 1/132 individuals (BAXTER et al., 2014; CENTERS FOR DISEASE CONTROL AND PREVENTION, 2012), with a 3-4:1 rate presenting more males than females, with a more balanced ratio if patients present dysmorphic features (BOURGERON, 2015; MANNING-COURTNEY et al., 2013). Recent reports achieved a prevalence of $1.68 \%$ in the United States, and in some places, like North Carolina, the percentage was estimated at $3.62 \%$. It has been argued that this increase in prevalence or the difference among sites could be related to discrepancies in survey designs and the participation of parents of children that never received an ASD diagnosis (FOMBONNE, 2018). In 1990, ASD prevalence was at $1 / 1000$, but this 10fold increase in prevalence is not distinguishable from the changes in ascertainment to a true increase in prevalence (ANAGNOSTOU et al., 2014).

Since it is a relatively common disorder, any couple in the population runs the risk of matching the prevalence of the disease for that population (around 1-2\% worldwide). As a result of its genetic component, with many population studies placing ASD heritability as being higher than 0.5 (COLVERT et al., 2015; LICHTENSTEIN et al., 2010; SANDIN et al., 2014; 2017), it is estimated that almost $50 \%$ of the simplex families are part of the high-risk class in which an inherited cause could be predominant (RONEMUS et al., 2014). The recurrent risk for the families with one child diagnosed is about $18.7 \%$, and for those individuals with two older siblings this risk goes up to $32.3 \%$ (WOODBURY-SMITH; SCHERER, 2018).

Family studies show that ASD can segregate according to all possible modes of inheritance (DE RUBEIS; BUXBAUM, 2015) and is compatible with what is known as complex diseases. Some authors use the term Complex Disease as synonymous with diseases with multifactorial or polygenic modes of inheritance (MITCHELL, 2012), although both represent very distinct genetic basis. Both modes of inheritance necessarily involve the action of several genes together, compatible with additive or epistasis models, and the main difference between them is that the multifactorial model adds the environmental component, while the polygenic model adds only the relative expression of each gene (RIDLEY, 2009). Complex Disease, can also be interpreted as a pattern of inheritance that can include all modes of inheritance together, which means including in the same term the mendelian, multifactorial and polygenic modes of inheritance (MITCHELL, 2012; SCHORK, 1997). In the case of ASD, this more inclusive definition for complex disease can be widely applied, and the results of biological and statistical approaches would be more successful when all the possibilities of inheritance are found in the individual in question (LA TORREUBIETA et al., 2016). 


\subsection{Molecular aspects of autism spectrum disorder}

After the availability of high-throughput microarray and sequencing platforms much advances have been made to the understanding of ASD genetic risk, nowadays it can be considered that the genetic basis of ASD is better understood, even in the absence of a resolutive molecular test for the diagnosis (WOODBURY-SMITH; SCHERER, 2018).

Copy number variation (CNV) and single nucleotide variants (SNV) are both implicated, with a strong confirmation in literature for the de novo highly disruptive variants (IOSSIFOV et al., 2012; O'ROAK et al., 2011; RONEMUS et al., 2014; SANDERS et al., 2012; TURNER et al., 2017) along with rare variants in many genes related to ASD, showing pleiotropy and variable penetrance (TORDJMAN et al., 2014; WEINER et al., 2017; WOODBURY-SMITH; SCHERER, 2018). Autism Spectrum Disorders' heritability has been estimated from 0.5 to 0.9 (LICHTENSTEIN et al., 2010; SANDIN et al., 2014; 2017), this estimative is suggestive of a strong genetic component for ASD. For ASD no genetic variation alone accounts for more than $1 \%$ of the cases, and even individuals with identical genetic variants demonstrate a large degree of phenotypic variability. In this manner the strong genetic component should be related to a highly variable genetic background (BOURGERON, 2015; CHAHROUR et al., 2012; LA TORRE-UBIETA et al., 2016).

CNVs, both deletion and duplications above $1 \mathrm{kbp}$, are frequent in patients with ASD (GESCHWIND; STATE, 2015), mainly involving specific regions of chromosomes 1, 2, 3, 5, 7, 11, 12, 13, 15, 16, 17, 18, 21, 22 and X (SCHAEFER; MENDELSOHN, 2013), with special attention to 16p11.2 (affecting $0.8 \%$ of individuals with ASD), 15q11-13 (0.5\%) and 22q11 (0.5\%) (GESCHWIND; STATE, 2015; MOREIRA et al., 2016; WOODBURY-SMITH; SCHERER, 2018). These variants were found in about ten-times excess in simplex families compared with typical controls (BRANDLER et al., 2015; SEBAT et al., 2007), and early studies showed that CNVs disrupted the locus containing SHANK3 and other promising candidate autism risk loci (SZATMARI et al., 2007). Also, 10\% of the individuals with an established pathogenic CNV carried a second large one, and $72 \%$ of the second CNVs were inherited from the mother, while the primary CNV (de novo or inherited) had no parental transmission bias (GIRIRAJAN et al., 2013). These studies converge on an estimate that around 5-15\% of individuals with a diagnosis of ASD will carry a CNV conferring substantial risk, versus $1-2 \%$ of the general population (BOURGERON, 2015; GESCHWIND; STATE, 2015).

Single nucleotide variants (SNV) have also been recognized, but to a lesser extent and for the majority of cases without a well described penetrance of the phenotype related to the mutations (SCHERER; DAWSON, 2011; WOODBURYSMITH; SCHERER, 2018).

The overall rate of de novo SNV in ASD was slightly elevated when compared with unaffected siblings, and two to three-fold predicted to be more disruptive than the ones carried by the siblings (DE RUBEIS et al., 2014; IOSSIFOV et al., 2014; NEALE 
et al., 2013; O'ROAK et al., 2011; O'ROAK et al., 2012a; RONEMUS et al., 2014; SAMOCHA et al., 2014). The majority of de novo point mutations in ASD originate from the father, with the correlation between paternal age and number of de novo events reflecting a 1.3-fold increase in the number of de novo events for every 10 years of paternal age (RONEMUS et al., 2014). The de novo likely gene disruptive mutations appears more often in genes expressed in the brain and genes already classified as candidate (CUKIER et al., 2014; SANDERS et al., 2012; YUEN et al., 2016).

Some studies show that transmission of probably causative variants originates most often from the mother, and transmission of such variants is seen more often in offspring with lower IQ (IOSSIFOV et al., 2015; KRUMM et al., 2015; STESSMAN et al., 2017). These findings are compatible to what first have been described for CNV (JACQUEMONT et al., 2014) and is in concordance with a model of female protective background for rare moderately penetrant mutations ( $\mathrm{SNVs}$ and $\mathrm{CNVs}$ ). There are more inherited SNVs that disrupt protein function in conserved genes transmitted from the mother to individuals with ASD than in unaffected siblings. However, there are contradictory research regarding this same finding for the CNVs (LA TORREUBIETA et al., 2016).

The percentage of the genetic diagnosis from likely gene disruptive variants in ASD can vary depending on the study and it is around 5-15\% (IOSSIFOV et al., 2015; SANDERS et al., 2012). A model for the disease with several high penetrance genes could not explain the network data found which can lead to the conclusion that a single deleterious event is unlikely to fully explain the disease in most cases (NEALE et al., 2013).

The fact that many families segregating ASD also seem to share risk for other neuropsychiatric disorders suggests that this genetic risk may be more correctly ascribed to fundamental brain-based traits (WOODBURY-SMITH; SCHERER, 2018). The number of genes estimated to be responsible for "monogenic" forms of ASD is between 400-1000 (BOURGERON, 2015; IOSSIFOV et al., 2015; LUO et al., 2018). The pathophysiology of the disorder is related to the enrichment of de novo events at specific genes and pathways.

In addition to the findings described previously, evidence supports a substantial contribution of common genetic variation to ASD (GAUGLER et al., 2014; GROVE et al., 2017; WOODBURY-SMITH; SCHERER, 2018), nevertheless most loci identified, even those with genome-wide significance, were not replicated in subsequent studies (ABRAHAMS; GESCHWIND, 2008; WEISS et al., 2009). Even with the estimated $40-60 \%$ of the genetic risk for ASD from the common variants (GAUGLER et al., 2014; KLEI et al., 2012; WEINER et al., 2017), studies of inherited forms of ASD need a higher number of samples to have sufficient power to identify and replicate common alleles at genome-wide significance (SCHIZOPHRENIA WORKING GROUP OF THE PSYCHIATRIC GENOMICS CONSORTIUM, 2014).

Taking into account the types of variants and genes related to the etiology of ASD there are many models being proposed to explain its pathophysiology. The synaptic homeostasis hypothesis for ASD suggests that genetic variants could either increase or reduce the number or strength of the synapses' connections and lead to the 
emergence of the phenotype, with common single nucleotide polymorphisms acting as a buffer for deleterious rare variants and unaffected individuals between these two extremes (BOURGERON, 2015). Many studies have confirmed the role of disrupting synaptic genes for the etiology of ASD (DE RUBEIS et al., 2014; PINTO et al., 2014; ROTSCHAFER; MARSHAK; CRAMER, 2015).

Luo et al. 2018 proposed a complex model involving two modules interconnected by the MAPK pathway. Module I includes Wnt signaling, cell adhesion, junction and cytoskeleton-related pathways, while module II includes glutamatergic synapse, GABAergic synapse and related process. In this model a double hit of gene + pathway is necessary for the development of the full phenotype, which means a less importance for the burden theory, which favors single hits in genes recurrently mutated in ASD. They built a more inclusive dual-hit model, where the disease variants need to disrupt the target genes and hit the relevant pathways. This model better explains ASD risk than the well-accepted mutation burden model (LUO et al., 2018).

The amplification of the investigation for the whole genome sequencing did not reveal a great impact of regulatory non-coding variants (WERLING et al., 2017), but instead confirming findings of candidate genes (C YUEN et al., 2017; YUEN et al., 2015). Recently Bradler et al. (2018) investigated possible roles for the regulatory regions in the etiology of ASD, like the cis-regulatory structural variants (BRANDLER et al., 2018).

Nevertheless even with the main discoveries regarding ASD genetic basis there is no laboratory test that can return a reliable specific result for $70 \%$ of the families. In this sense it is possible to hypothesize what types of mutations are important for the diagnosis of ASD, and perhaps construct a more robust test to provide a molecular diagnosis for the families. It is always important to keep in mind that each of these models explains a portion of the disease and that the only certainty is that the ASD is not caused by a single model of transmission (PINTO et al., 2014). In site of these models of ASD etiology it is difficult to establish which is the scenario for the specific individual so it can be programmed a treatment or genetic counseling.

The importance to discriminate between complex and essential ASD (essential refers to the presence of isolated characteristics of ASD, and complex refers to the individuals with the co-occurrence of other morbidities) is because the presence of specific comorbidities increases the probability of a diagnostic hypothesis that can be evaluated by a clinical geneticist to establish the most effective molecular test (SCHAEFER; MENDELSOHN, 2013; WOODBURY-SMITH; SCHERER, 2018).

The American College of Medical Genetics and Genomics (ACMG) 2013 guideline for the molecular investigation of autism is still in agreement with the recent literature (SCHAEFER; MENDELSOHN, 2013; WOODBURY-SMITH; SCHERER, 2018). Penetrant single variants (CNV, chromosomal rearrangement, short indel or SNV) is calculated to happen in 10-25\% of the individuals with ASD (BOURGERON, 2015). The CNV findings are believed to be responsible for the majority of the cases with a molecular diagnosis, so a molecular investigation with microarray techniques is indicated to detect these alterations as a first approach to a patient with ASD 
(KRUMM et al., 2013; SCHAEFER; MENDELSOHN, 2013).

In this context, $\mathrm{ACMG}$ suggests that there is no formal indication for the specific test of each gene when suspected ASD (SCHAEFER; MENDELSOHN, 2013). Despite the constant registration of new candidate genes, the finding of a single disrupting variant in a candidate gene is not sufficient to provide a molecular diagnosis, which encourages searches for complex relationships between variants in the same individual and opens space for exploring new hypotheses regarding their etiological bases (VAISHNAVI et al., 2014). Without a highly definitive molecular diagnosis for ASD, the best predictor of sibling recurrence remains family history (GESCHWIND; STATE, 2015).

\subsection{Next Generation Sequencing and the Diagnosis of Rare Diseases}

With the advent of the Next Generation Sequencing (NGS) techniques, it became possible to investigate gene alterations on a large scale, with the advantage of being able to observe many genes at the same time, without the need to choose which candidate gene would be preferably researched (MARDIS, 2008). This methodology made the sequencing of the human whole genome cost-effective and increased the appeal to apply this technique to investigating rare diseases (LAPIN et al., 2016).

So that this methodology is made even more cost-effective, it is possible to sequence only the coding regions of the genes, the exons (whole exome sequencing WES). It is estimated that $85 \%$ of the mendelian diseases that have known genetic etiology could be diagnosed by using this approach (TRUJILLANO et al., 2016) by sequencing only $2 \%$ of the genome, which means a great reduction in the amount of data sequenced (MATULLO; DI GAETANO; GUARRERA, 2013).

Despite these high estimations, about $31 \%$ of the exomes sequenced in a clinical laboratory had a positive gene finding, $25 \%$ had a variant of unknown significance, and $45 \%$ had no relevant variant, and these rates can vary depending on the diseases being investigated (TRUJILLANO et al., 2016). This difference in numbers occurs because more than 3,000 known mendelian diseases do not have a genetic etiology established (CHONG et al., 2015).

A single WES generates approximately 30,000-50,000 variants that are different from the human reference genome (LAPIN et al., 2016). Each individual carries about 150 loss-of-function variants and 2,500 nonsynonymous (MATULLO; DI GAETANO; GUARRERA, 2013), of which 281-515 are predicted by in silico tools to be highly damaging and 40-85 to be in homozygous state (XUE et al., 2012). This number of variants makes it difficult to determine which ones may be related to an effect on the development of the central nervous system to the point of generating the pathological phenotype. Theoretical and experimental studies suggest that individuals typically carry hundreds of mildly disadvantageous variants and several severe disease alleles 
(XUE et al., 2012).

Most living humans share large segments of genetic variation, especially because they are the progeny of rapid expansion of human populations from a relatively small number of remote ancestors (about 10,000 years ago) (RAMACHANDRAN et al., 2005). This scenario can lead to interesting population effects, with the accumulation of new mutations and rare alleles that did not have enough time to be removed by natural selection (MATULLO; DI GAETANO; GUARRERA, 2013), and, if not taken carefully, can lead to false positive results in the clinical-laboratory context and misconduct by the physician in charge (MANRAI et al., 2016).

Two processes are fundamental for achieving the final result in molecular investigation of genetic diseases, and gene and variant prioritization (EILBECK; QUINLAN; YANDELL, 2017). Both processes can be carried out with the aid of bioinformatics and clinical knowledge. Information, such as predicted damage to the protein, population allele frequency, functional testing, presence of the variant in other individuals with same phenotype, segregation in the family and mode of inheritance can be used to classify variants and give a final result (RICHARDS et al., 2015). The misclassification of variants can have a high impact leading to false positives or false negatives in the clinical practice. As an example of the possible extension of those errors, the ExAC database was constructed with approximately 65,000 WES conducted in adults without severe genetic conditions. An average ExAC participant harbors close to 54 variants reported as disease-causing in two other databases, an implausibly high number, even considering that these individuals can be affected by several polygenic disorders (LEK et al., 2016). This can have a high impact on the choices of candidate genes for genetic diseases depending on the population assessed.

In rare monogenic diseases, NGS is a tool that follows the precepts of a diagnostic examination, making a clear distinction between the group of affected and that of non-affected individuals, and can be used as a first diagnostic choice (TRUJILLANO et al., 2016). However, in complex diseases of high prevalence, quality parameters for WES have yet to be well established. Most of the dominant or recessive mendelian disorders are caused by rare mutations (with frequency under 0.01 in the population), which is consistent with the low frequency of these conditions in most populations. Nonetheless, in general, for genetic conditions, common variants (with frequency above 0.01$)$ with large disease effect sizes $(>2.0)$ are exceptional: most known common risk alleles have been shown to increase susceptibility by less than 1.5-2.0 times (GESCHWIND; STATE, 2015). Laboratory tests for diagnosis should have high accuracy, and lead to a minimum number of false positives and false negatives. The excess of variants present in each individual can become a major obstacle to the research.

It is therefore important to consider the probability of damage and that of phenotype penetrance of candidate variants when attempting to counsel families about the risk. For children with positive genetic test results, genetic counseling offers the opportunity to plan a treatment and/or prophylactic measures for any medical condition known to be associated with the particular gene (WOODBURY-SMITH; 


\subsection{Next Generation Sequencing and the ASD Diagnosis}

Analysis of the coding regions of the genome by whole exome sequencing (WES) has detected several candidate genes and etiological agents of Autism Spectrum Disorder (ASD), and has also been effective in identifying some variants with probable oligogenic inheritance (GUO et al., 2011). However, even with the current innovative techniques, the number of cases elucidated still remains very low, since the mutations found usually refer to genes not previously described in ASD, and with low recurrence in affected individuals (YU et al., 2013).

There is still no evidence that WES can be used as a clinical-laboratory test for ASD, and, despite all the main advances that this technique has made for understanding ASD within the scientific context, little was done to test whether WES could achieve a high level of sensitivity or level of specificity for each individual in clinical-laboratory context. Most studies focus on comprehending the pathophysiology and proposing scenarios or candidate genes (HE et al., 2013; LIU et al., 2014), but some few focus on discriminating the individuals and proposing a physiological explanation for the disease in a particular subject.

It is possible to observe that, since efforts to find a single cause did not bring the expected resoluteness, the research groups turn their work to the search for general hypotheses involving more than one mode of inheritance and more than one associated gene to explain ASD etiology. So far, these questions have brought countless answers, but every one of them presented restricted resolving power (CHAHROUR et al., 2012; CUKIER et al., 2014; KUMAR, 2011; O'ROAK et al., 2011; SANDERS et al., 2012; YU et al., 2013), without taking into account that, for the affected individual, any of the hypotheses described may be true. Therefore, a multivariate approach is required for these hypotheses, in which individuals can be analyzed as a whole in the face of all possibilities.

Genetic models for complex diseases, such as ASD, are very hard to establish, particularly because the phenotype can be caused by all possible inheritances. Fully penetrant genes, like PTEN and SHANK3, account for less than $0.5 \%$ of the ASD cases, other genetic variants, such as the $15 q 13$ microdeletion/duplication, have a higher rate, but they are not fully penetrant (LA TORRE-UBIETA et al., 2016). Also, scenarios were the combination of rare high-risk and common low-risk variants can lead to the phenotype (WEINER et al., 2017).

This evidence for genetic heterogeneity among individuals and even within the same family presenting more than one individual affected, along with pleiotropy, speaks to the need for precision medicine, a personalized approach to genetics that is cognizant of the fact that assessment of an individual's complete genomic risk is 
required (WOODBURY-SMITH; SCHERER, 2018).

These are the challenges of any complex disease and the reason why the initial analysis of a candidate group of genes can be exhaustive. This study aims at making this first approach easier and at quickly detecting whether candidate genes could be used to discriminate the affected group from the control group, and how it would be done by using whole exome sequencing. The approach to count the variants and to sort them into flexible categories depending on the initial hypothesis is a way to access burden of types of variants in specific genes or pathways. If ASD is caused by the accumulation of specific types of variants in determined genes than it is expected that individuals without ASD would present a different pattern for the number of variants for the same genes.

This thesis can be divided into two parts, one for developing the pipelines and another for interpreting the results they provide. The subject of interpretation can be further divided into interpreting each report individually, the impact this analysis has on the laboratory that interprets the whole exome sequencing, and, finally, the results in the context of the etiology of the autism spectrum disorders. 


\section{Goals}

The main goal of this study was to evaluate a series of tests to maximize the molecular diagnosis for autism spectrum disorder with whole exome sequencing in the clinical-laboratory reality. The tests would involve direct approaches as well as multivariate analysis based on the quantity of variants presented by each individual, applying statistical tests to evaluate the discriminatory power between affected and control groups.

The specific goals were:

- $\quad$ - To identify potential biases for the analysis involving subjects and sequencing strategies, and to identify good strategies to overcome the biases losing the lowest number of individuals possible.

- $\quad$ - To develop a pipeline to count the number and types of variants filtered for specific gene lists for each individual from a whole exome sequencing, by taking into account the quality of the predicted variant.

- - To automatically test gene lists with different mathematical approaches and display the results into a report of friendly interpretation.

To identify potential discriminant strategies for autism spectrum disorder with whole exome sequencing that would achieve higher levels of sensitivity and of specificity than the current approach. 


\section{Material and Methods}

This section is divided into four parts, where the first describes the choice of the individuals to be used in the study; the second describes the gene lists used to test the different approaches to discriminating the ASD group from the control group; the third describes sequencing methods and annotation of each individual; and the fourth describes the statistical approaches used to develop the final report for each list. The project of this thesis received the approval of the Ethics Committee from Instituto de Biociências da Universidade de São Paulo (IB-USP), under number 62602016.3.0000.5464.

\subsection{Subjects}

\subsubsection{Austism Spectrum Disorder Subjects}

We first started with 71 individuals with clinical diagnosis of autism spectrum disorder (ASD), including 54 different families and 34 trios. In order to simulate the reality of a clinical genetic laboratory, and to avoid biased results due to the use of family members, we decided to continue the analysis with 54 probands, 14 other family members diagnosed (multiplex), and 40 from simplex families.

Six individuals were removed from these 54 due to sequencing quality issues, and the remaining 48 individuals were sorted into 37 simplex cases and 11 multiplex cases. 25 of these 48 individuals were syndromic and the other 33 cases were either nonsyndromic or lacked additional information.

\subsubsection{Control sample}

The choice of the individuals for the control sample followed important steps to avoid bias. Two different approaches were tested, one with the 609 healthy individuals older than 60 years old, and the other with 175 individuals presenting other clinical diagnosis, such as rasopathies, skeletal dysplasia and cleft lip and palate. All the individuals were sequenced in the Centro de Estudos do Genoma Humano da Universidade de São Paulo (CEGH-USP) under other scientific projects and are representative of the São Paulo and Brazilian ancestry (BERTOLA et al., 2014; NASLAVSKY et al., 2017; YAMAMOTO et al., 2014).

With the first attempts at differentiating the groups with Principal Components Analysis (PCA) (more detail in further sections of Material \& Methods), it was 
observed that the sequencing batch had a strong influence on the PCA results, as seen on Figure 1. Changes in bioinformatics pipeline to produce the variant call format (VCF) files had to be established to overcome the effects different sequencing had on rare variants (more details in further sections). Figure 2 shows an example of the kind of effect different VCF files can have on the final result.

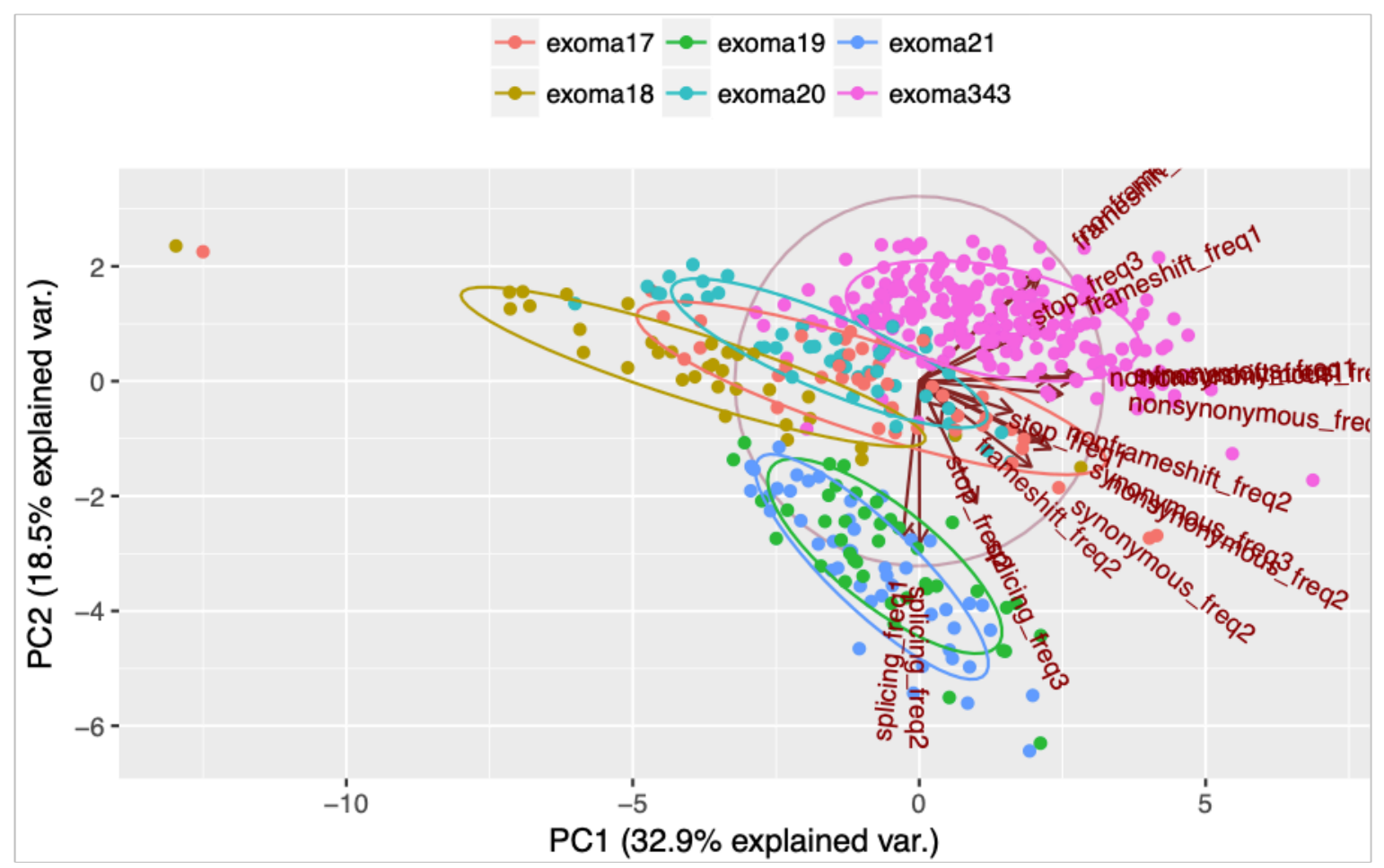

Figure 1: First two PC from different sequencing batches, from 17 to 21 , and the pink (exoma343) with 343 individuals called together.

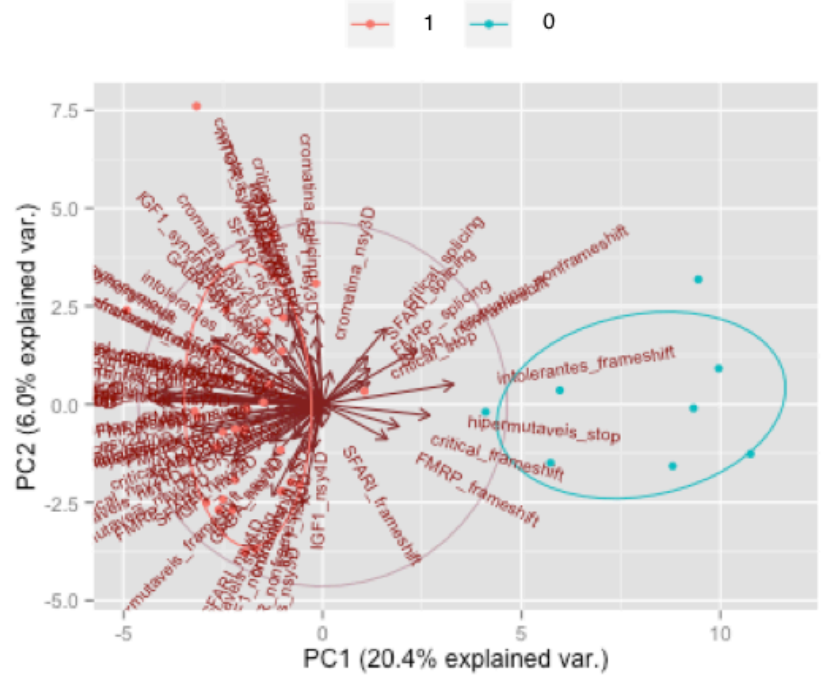

Figure 2: The first two PCs with only the ASD probands, in red (1), and the probands with cleft lip and palate, in blue (0), using all rare variants, before the changes in the pipeline to produce a unique VCF file. 
After the adjustments to make a unique VCF file, the PCA from all variants still resulted in a complete separation from the groups, specially separating the 609 elderly healthy samples, as it can be seen in Figure 3.

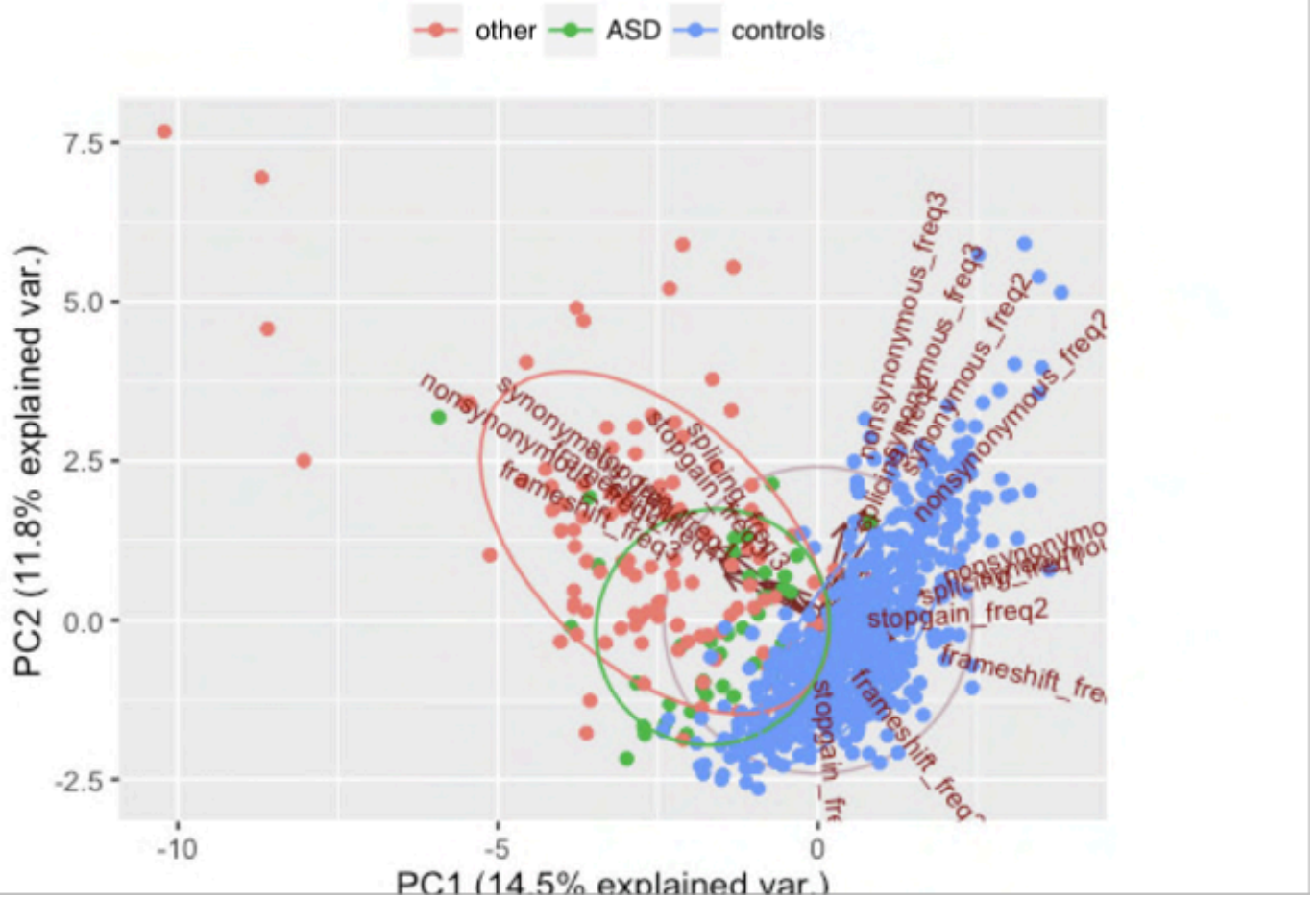

Figure 3: The first two PCs for the three groups, red represents the control group with other diagnosis, green represents the ASD group, and blue represents the 609 healthy control individuals.

Based on the results published in literature, it was not expected to see this clear separation among the samples, specially between the ASD group and the healthy control group. This kind of phenomena can lead to false positive results, and overfitting models. Figures 4 and 5 are examples of overfitting models. Both show the receiver operating characteristic (ROC) curve from a logistic regression model based on variants from VCF files after corrections to the pipeline (more detail in further sections of Material \& Methods). While Figure 4 shows no discrimination between the ASD group and the control group (175 individuals with other diagnostics), Figure 5 shows the same dataset comparing with the 609 healthy individuals, revealing a high degree of discrimination between the groups, which, based on the scientific literature published so far, was not expected. 


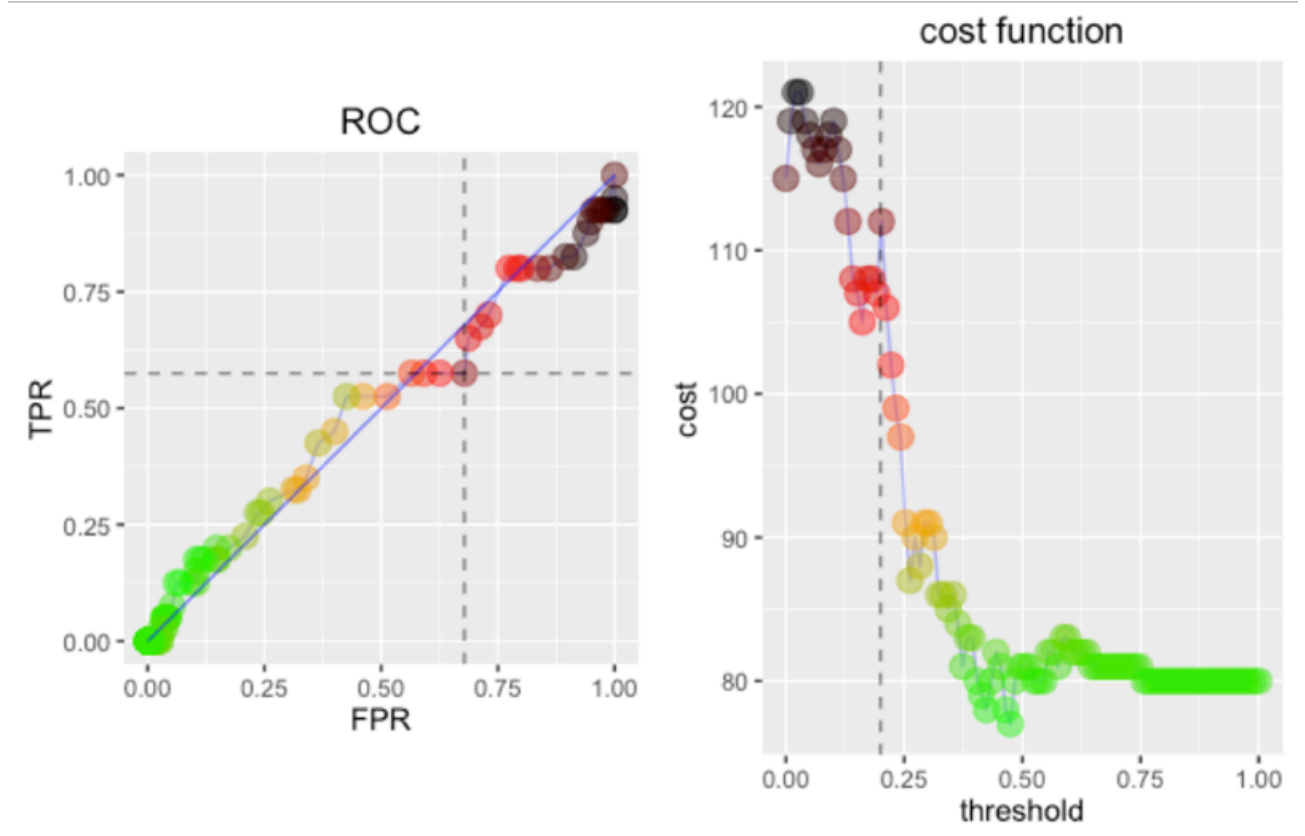

Figure 4: ROC curve of all variants comparing the results of a logistic regression between ASD and other disease groups.

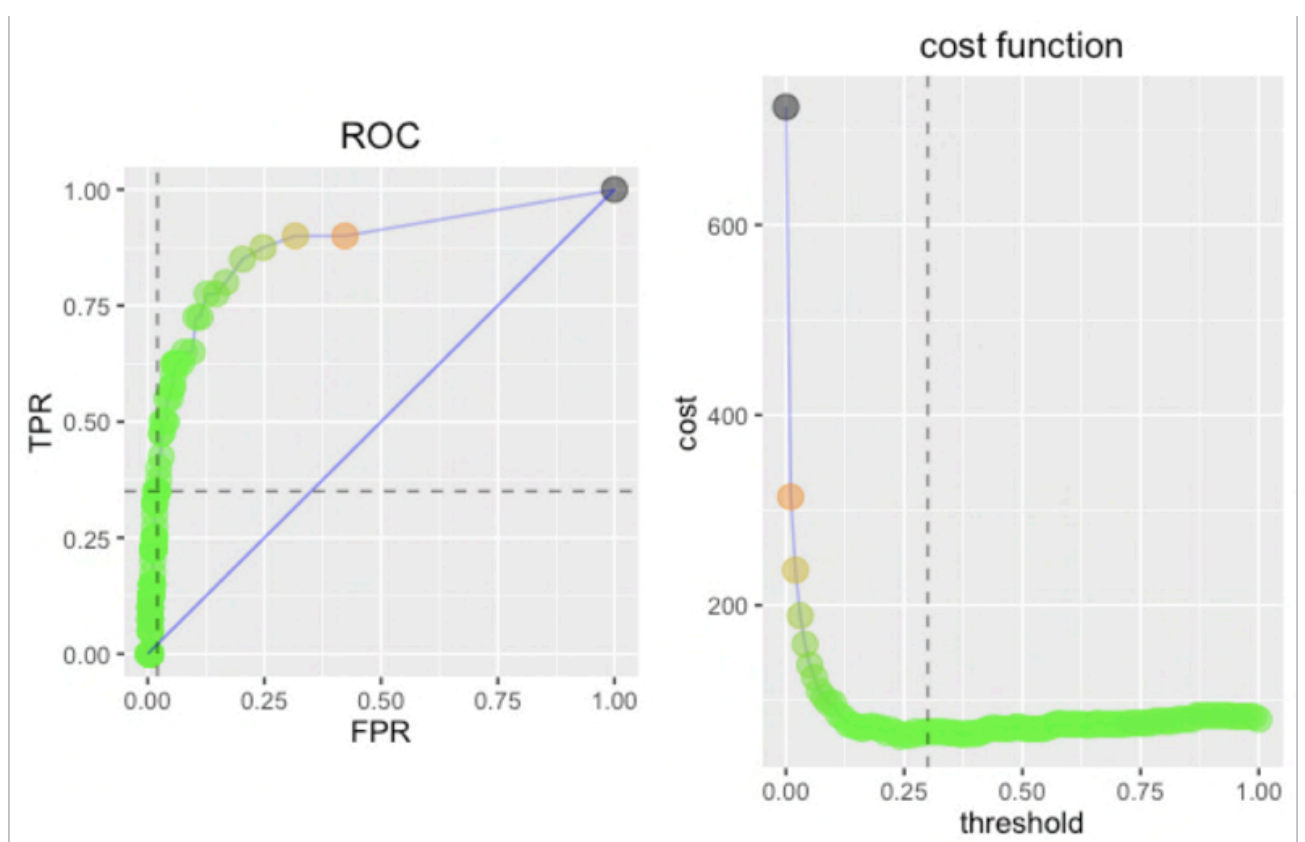

Figure 5: ROC curve of all variants comparing the results of a logistic regression between ASD and healthy individuals.

The 119 control individuals present different diagnosis and are sorted into the following (Table 1) diagnosis.

Table 1: Description of the diagnosis in controls

\begin{tabular}{|l|l|}
\hline Diagnosis & Individuals \\
\hline Acrofrontofacionasal & 1 \\
\hline Auriculo condilar & 1 \\
\hline
\end{tabular}




\begin{tabular}{|l|l|}
\hline Cardiofaciocutaneous & 10 \\
\hline Nonsyndromic cleft lip and palate & 5 \\
\hline Syndromic cleft lip and palate & 1 \\
\hline Condrodisplasia punctata & 1 \\
\hline Craniossinostose & 8 \\
\hline Skeletal dysplasia & 7 \\
\hline Unknown & 3 \\
\hline Spondyloepyphyseal dysplasia & 1 \\
\hline Amyotrophic Lateral Sclerosis & 4 \\
\hline Spondylocostal & 1 \\
\hline FG Syndrome & 1 \\
\hline Frontonasal & 1 \\
\hline Neurofibromatosis & 2 \\
\hline Noonan & 57 \\
\hline Oculoauriculovertebral & 1 \\
\hline Oculoauriculofrontonasal & 1 \\
\hline Sener & 1 \\
\hline Infection by Zika virus & 11 \\
\hline
\end{tabular}

This result can be related to a bias generated by the type of sequencing and bioinformatics the 609 healthy individuals were submitted to. Thus, only the probands of the individuals with other diagnosis, 119 individuals in total, were used for subsequent analysis. In short, this work was done with 48 ASD cases and 119 control individuals, resulting in 167 individuals.

\subsubsection{Kinship and ancestry analysis}

Focusing on 23,556 of pre-selected single nucleotide variants (SNV), multiple fast quality and kinship control were computed for the 168 individuals in this analysis, with the software Peddy developed by Pedersen and Quinlan (2017).

The analysis of the ratio of heterozygous calls by the median depth of coverage showed all 168 individuals with a proportion of heterozygous between 0.27 and 0.35 , with no outliers with an excess in heterozygous variants that can happen in situations of sample contamination or high degree of sequencing errors (Figure 6) (WANG et al., 2015). All sequencing had at least $80 \%$ of the target regions covered with a minimum of 10x, a pattern considered of high sequencing quality by the ExAC consortium for population studies (LEK et al., 2016). 


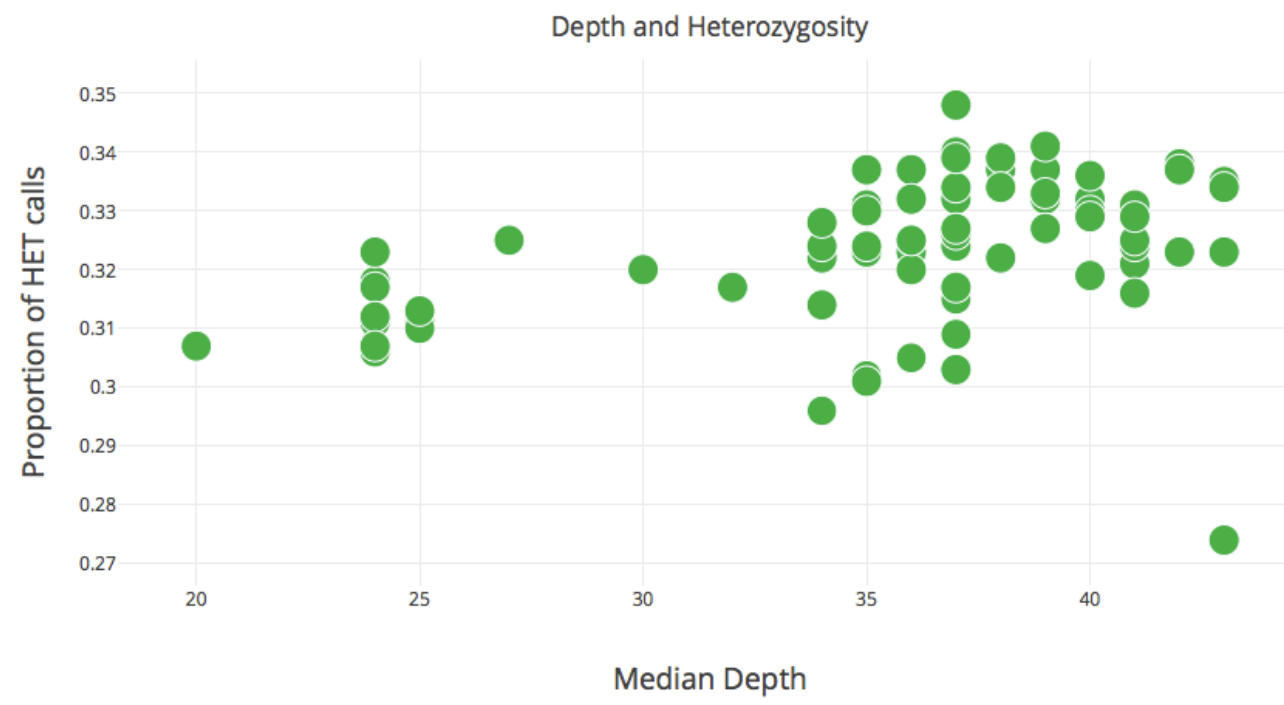

Figure 6: Scatterplot with the proportion of heterozygotes by the median depth of coverage in WES, for each individual in the analysis.

To confirm that the samples did not share genetic background (individuals could be considered as distinct families), the pairwise coefficient of relatedness (CR) for all samples was applied. Figure 7 shows the CR for each pair for the 168 individuals, all resulting in less than 0.05 relatedness, the same score expected for random individuals in the human population (PEDERSEN; QUINLAN, 2017).

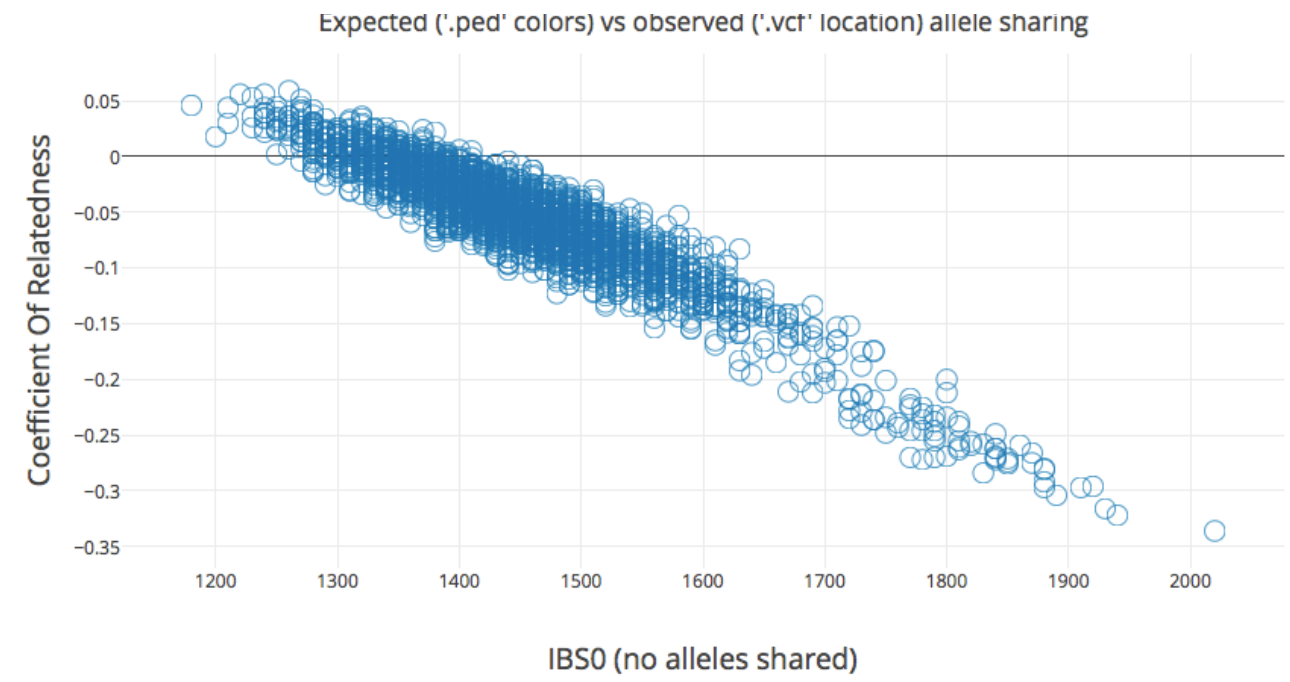

Figure 7: Scatterplot showing the coefficient of relatedness for the 168 individuals in the analysis.

Also, an analysis of the ethnic background was conducted for the samples. A principal component analysis based on informative SNPs from 1000Genomes project divided the world population into five categories: European, African, East Asian, South Asian and American. For our sample, the great majority of individuals were classified as American (Figure 8), for the individuals classified as unknown, European and African, their distributions were very close to the majority of the group and could 
be classified into the dispersion of Americans.

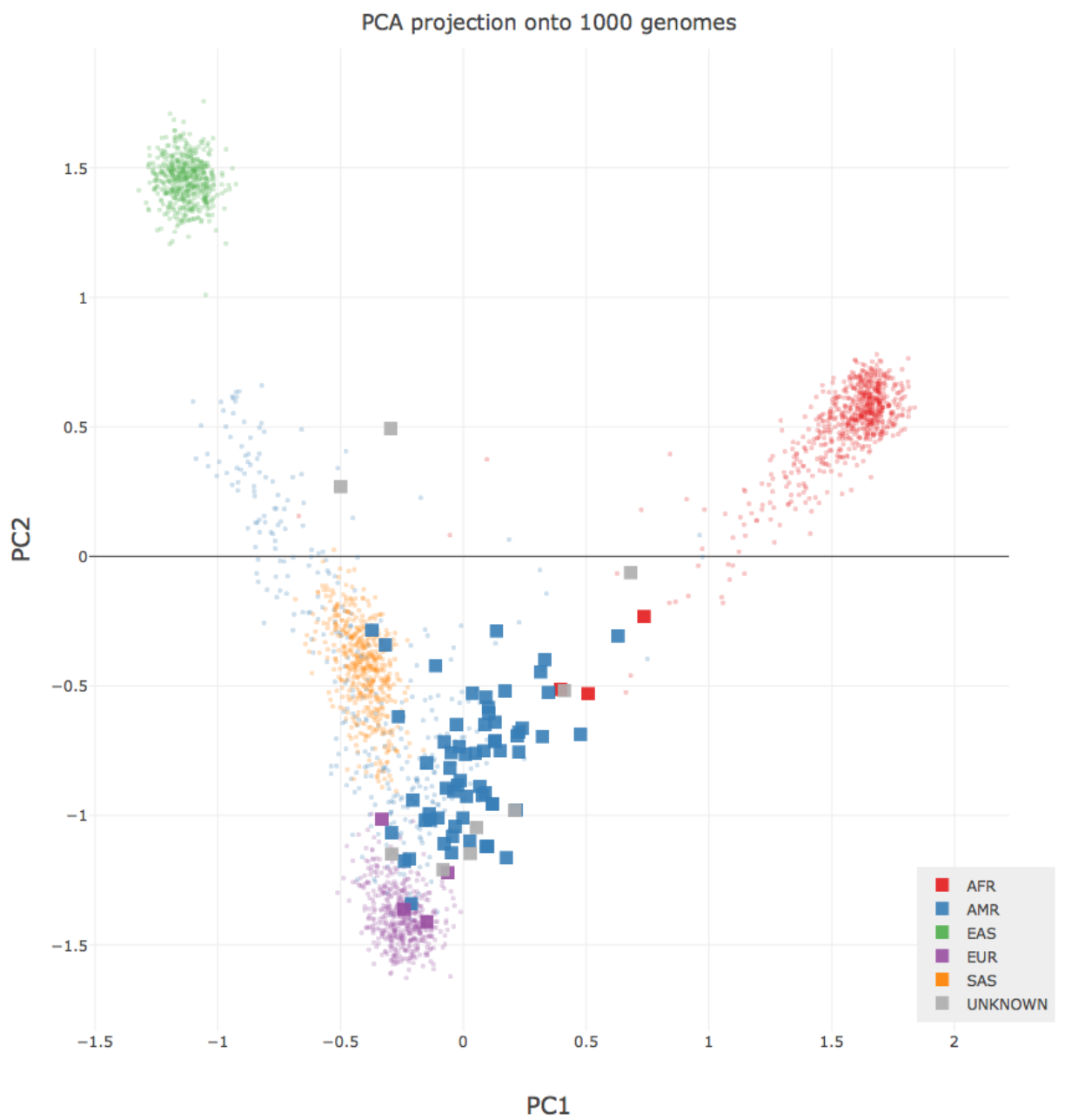

Figure 8: The first two PCs of the ancestry distribution based on 1000Genomes, the squares are the 168 individuals in this study.

\subsection{Gene lists}

Twenty-one gene lists were separated based on scientific publications and personal discoveries made by the group of Maria Rita Passos-Bueno, the lists will be described in detail further

\section{3.2.1 Genes with critical exons}

Uddin et al. 2014, compared the number of mutations in critical exons (exons with high expression in central nervous system and low rate of rare pathogenic 
polymorphisms) between ASD and control individuals. It has been observed that a greater proportion of these events occurs in individuals with ASD. In the study, they performed WES and transcriptome from different regions of brain tissue and observed 1,744 genes preferentially expressed in the brain containing 3,955 critical exons. They found that there was an accumulation of de novo mutations, predicted to be deleterious in the exons considered critical in individuals with ASD compared to control individuals. This study was quite effective in showing a possible way to molecularly discriminate individuals with ASD and unaffected individuals because the results tended to be extreme for both groups (UDDIN et al., 2014). This gene list can be seen on this article's supplement material and consists of 1,744 genes (Attachment 1).

\subsubsection{Chromatin modifier genes}

Cotney et al. in 2015 described the relation between CHD8 and other chromatin modifiers in ASD pathophysiology (COTNEY et al., 2015). This article, along with previous and recent articles, recognized the importance of de novo mutations in chromatin targets (KRUMM et al., 2015; O'ROAK et al., 2012a). These implications made the genes that are target to CHD8 or chromatin modifiers good candidates to test differences between ASD and control groups. The list of 263 genes included the genes described by Cotney et al. 2015 and the Reactome genes related to chromatin organization and signal transduction (Attachment 2).

\subsubsection{ASD-related genes}

Our group selected the gene list previously, based on the literature referring to ASD-related genes, and they were used to construct a customized array-CGH (MOREIRA et al., 2016) (Attachment 3).

\subsubsection{FMRP targets}

The most common recurrent cause of syndromic ASD is Fragile $X$ syndrome, which is caused by loss of the FMRP function (LA TORRE-UBIETA et al., 2016). As a result of this strict relation between FMRP and autism, it was hypothesized that mutations in FMRP targets could be related to ASD (DARNELL et al., 2011; STEINBERG; WEBBER, 2013). Darnell et al. 2011 identified 842 genes that were FMRP targets and Steinberg et al. 2013 found more enrichment of disruptive mutations in FMRP targets in the ASD group than in the control group. The gene list is found on 
Attachment 4.

\subsubsection{ASD-related genes}

Stessman et al. (2017) selected 208 ASD-related genes from published sequencing studies based on the number of de novo recurrence, overlap with CNV morbidity map, pathway connectivity and absence of de novo variants in 1909 unaffected siblings (STESSMAN et al., 2017). They describe 91 genes with excess de novo mutations or private disruptive mutations in $5.7 \%$ of individuals with ASD. This work was highly criticized by Barret et al. (2017) because of the flawed statistical approaches and correction for genome-wide scan for discovery genes (BARRETT et al., 2017). As a result of this recent dispute about the methodology of this list, we concluded that this could be a good subject for further discussion. The 208 genes are on Attachment 5.

\subsubsection{GABA pathway}

By taking into account that GABA is the main inhibitory neurotransmitter of the central nervous system and the fact that one of the most common deletions involved in ASD etiology is region 15q11-q13, which is enriched for genes encoding subunits of the GABA receptor, Cellot et Cherubini (2014) proposed a role that was further confirmed for this pathway in ASD etiology (CELLOT; CHERUBINI, 2014; HOGART et al., 2007; LA TORRE-UBIETA et al., 2016; LUO et al., 2018). The genes related to GABA reuptake, synthesis and degradation, GABA receptor activation and GABA modulators resulted in a list of 74 genes of interest (Attachment 6).

\subsubsection{Hypermutable Genes}

Hypermutable genes were regarded by Michaelson et al. (2012) as a possible source for ASD etiology (MICHAELSON et al., 2012). The hypermutable sites could be hotspot regions for disruptive mutations and could explain the high prevalence of autism. The article compared the genome sequencing in nine monozygotic twins and described many regions of hypermutability. They showed that the mutation frequencies varied widely by genome, with a difference of up to 100 times depending on the region, and this variation could be explained by the intrinsic characteristics of DNA sequence and chromatin structure. Many of these regions were related to previously described ASD genes. The supplemental material of this article gives a spreadsheet of the transcripts and their hypermutable rank for 1858 genes. For this 
work, only the genes with hypermutable scores above three in more than $50 \%$ of the transcripts were included and resulted in a list of 181 genes (Attachment 7).

\subsection{8 mTOR and RAS/MAPK Pathways}

Pinto et al. 2014 used the impact of new or inherited CNVs on 2,446 individuals with ASD and their families, and 2,640 control individuals to characterize candidate genes to be associated with ASD. They chose the genes disrupted by these CNVs and identified the biological relationship between them and the pathways they had in common. They have shown that both deletions and duplications involving the FMRP and post synaptic density genes increase the risk for ASD and genes present in CNVs

and SNVs with presumed loss-of-function converge on functional pathways, such as PI3K-Akt-TSC-PTEN-mTOR and PI3K-RAS-MAPK (BOURGERON, 2015; LUO et al., 2018; PINTO et al., 2014). In Reactome there were nine different pathways for mTOR. For this study, only mTOR signaling and the regulation of Rheb GTPase activity by AMPK were used. The total number of genes on this list is 39 (Attachment 8). The MAPK family signaling cascade, was also obtained from Reactome with 282 genes in total (Attachment 16).

\subsubsection{IGF1 Pathway}

Many recent studies show an impact of the treatment with IGF-1 on individuals with SHANK3 mutations (KOLEVZON et al., 2014; RIIKONEN, 2016; SHEMESH et al., 2012) and the possibility of using it as an ASD biomarker (KRUMM et al., 2015). Based on the importance of the mTOR pathway to ASD etiology, which is entirely contained in the IGF-1 pathway, and the possible roles described above, the IGF-1 pathway was considered for further study. The list was made with the Reactome signaling by IGF1R (type 1 insulin growth-factor 1 receptor) and consists of 303 genes (Attachment 9).

\subsubsection{Genes intolerant to loss-of-function mutations}

Many studies on de novo disruptive mutations in ASD show an enrichment of this type of mutation in affected individuals (IOSSIFOV et al., 2012; O'ROAK et al., 2011; RONEMUS et al., 2014; SANDERS et al., 2012). The article published by Krumm et al. (2015) showed the role loss-of-function de novo mutations play in ASD etiology (KRUMM et al., 2015) and concluded that genes that are more intolerant to loss-offunction mutation could play a major role in ASD etiology. From the 16,596 genes 
scored for intolerance by Petrovski et al. (2013), those with a residual variation intolerance score (RVIS) (PETROVSKI et al., 2013) higher than 50\% were enriched in ASD individuals. For this study only 7,764 genes achieved this threshol (Attachment $10)$.

\subsubsection{Differentially expressed genes}

One researcher from the group, Karina Griesi, is working with differentially expressed genes from ASD and control iPS cells. Her results generated five different sets of differentially expressed genes from a more relaxed search (M1) to a stricter one (M18). M1, M2, M8, M16 and M18 are described on Attachment 11, M2 in Attachment 12, M8 in Attachment 13, M16 in Attachment 14 and M18 in Attachment 15, respectively.

\subsubsection{SFARI}

The Simons Foundation Autism Research Initiative (SFARI) is a scientific initiative aimed at improving the understanding, diagnosis and treatment of ASD that involves more than 400 investigators in the U.S. and abroad. The SFARI has a gene scoring advisory panel that reviews literature on the genetics of autism, with emphasis on evidence from human genetics that separates the genes involved in the ASD etiology into 6 categories: syndromic, high confidence, strong candidate, suggestive evidence, minimal evidence and hypothesized but not tested. For the purpose of this work, only the first 4 categories were assessed, and the gene lists are as follows: syndromic (101 genes - Attachment 17), high confidence (23 genes -a Attachment 18), strong candidate (54 genes - Attachment 19), suggestive evidence (149 genes Attachment 20) and all of the three together (279 genes - Attachment 21).

\subsubsection{Comparison of the gene lists}

From the 21 gene lists only four genes (GRIN2B, GRIN1, STXBP1 and SLC6A1) are present in five lists, all the other genes are presented in four lists or less. Figure 9 presents all the genes analysed separated by each list. 


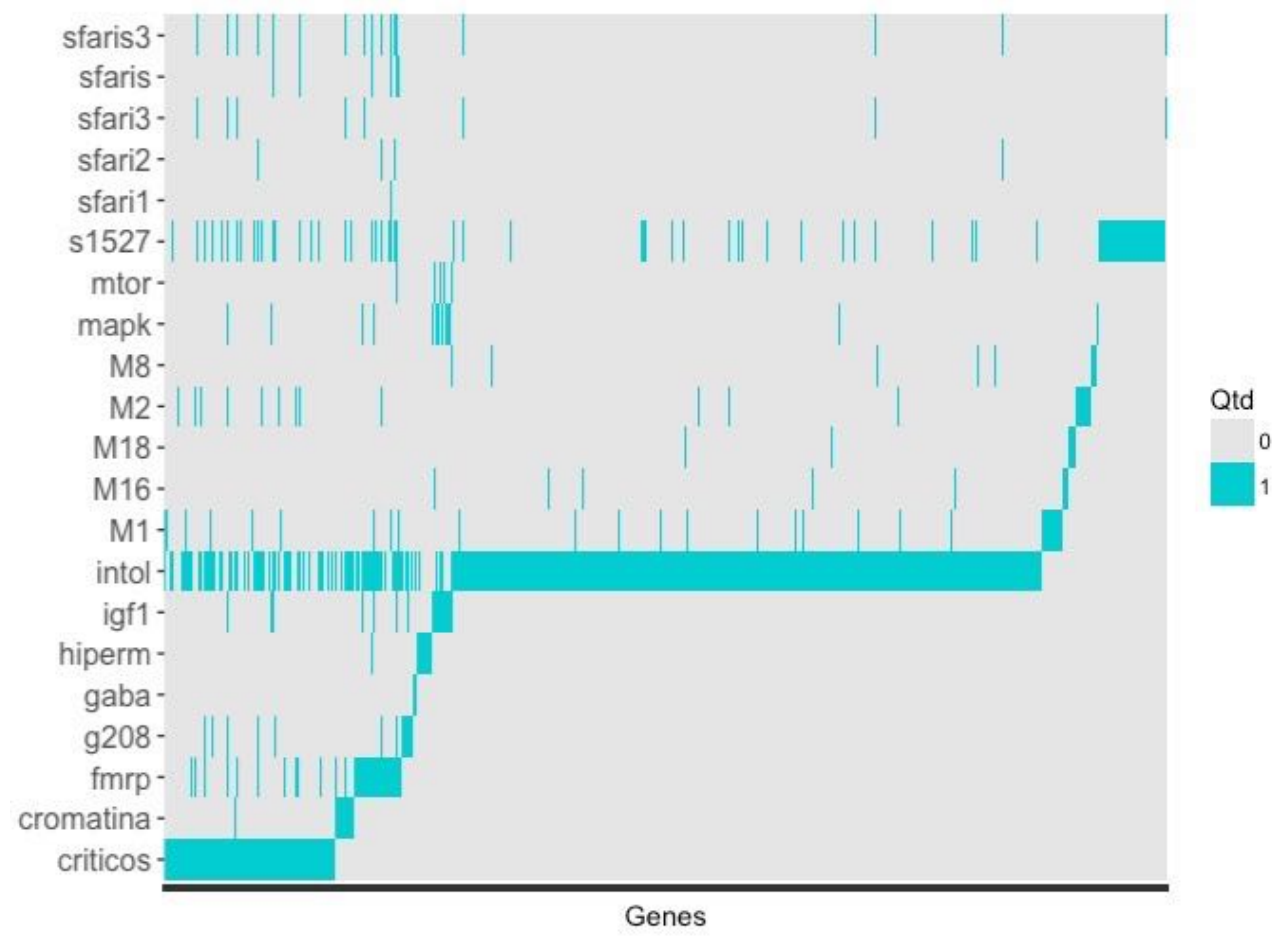

Figure 9: The representation of the redundancy of genes in all gene's lists. Each line in axis y is indicative of a particular gene.

Figure 10 shows the correlation of the genes by lists, the list with genes intolerant to loss of function mutations is highly correlated to almost all other lists, this phenomena could be explained by the number of intolerant to LoF genes and the fact that most other lists are made based on disruptive mutations present in individuals with ASD. Other group that has high correlation is the IGF1, mTOR and RAS/MAPK, this could be attributed to the fact that the mTOR and RAS/MAPK are pathways contained in the IGF1 pathway. The SFARIS3 is the union of the other SFARI lists and therefore has high correlation with them. 


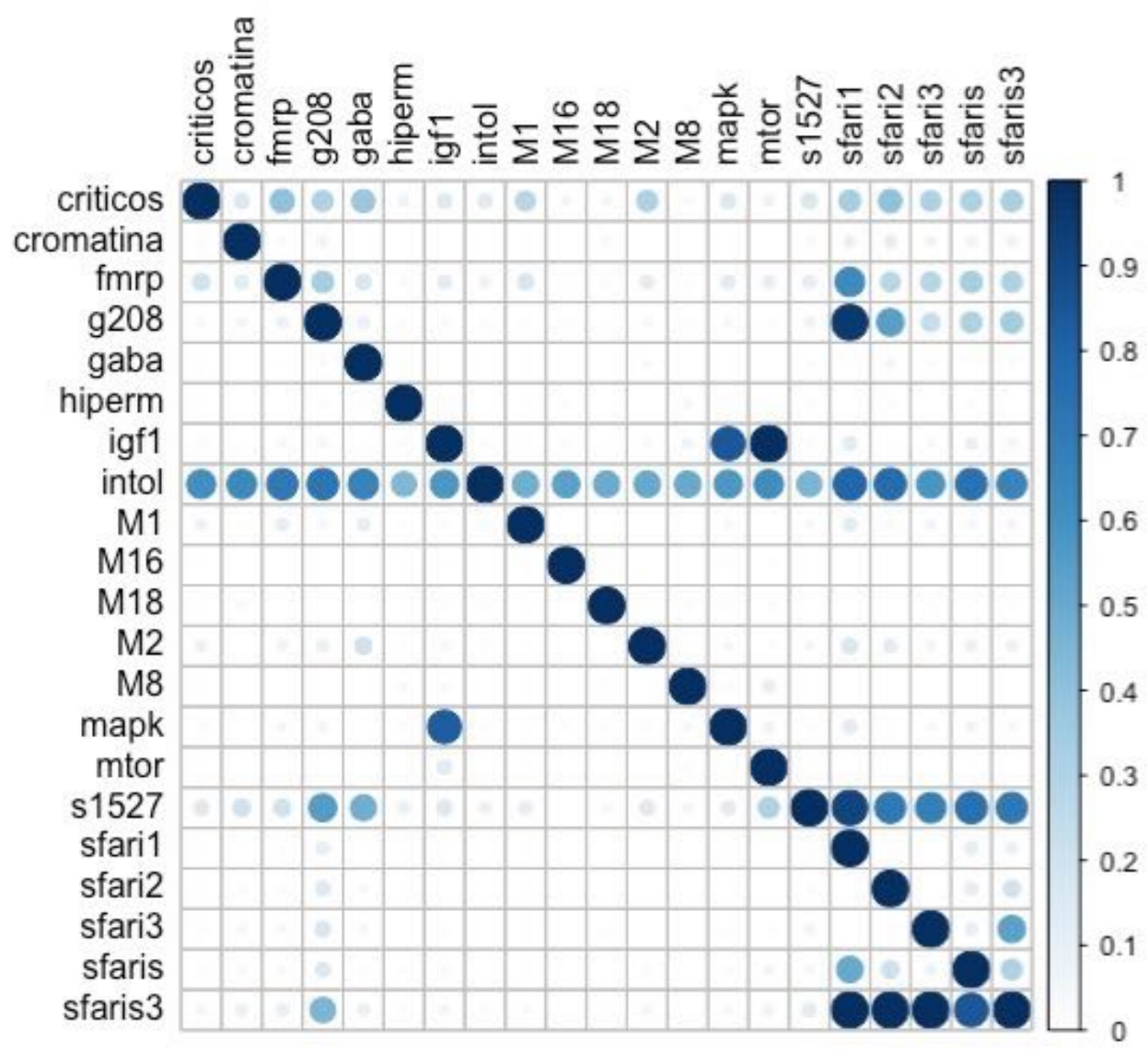

Figure 10: Correlation among the genes included in each gene list described.

\subsection{Sequencing and annotation strategies}

All the samples were sequenced by CEGH-USP from 2014 to 2017 with an Illumina's HiSeq2500 sequencer. Twenty-one batches of exomes were sequenced, the numbering followed a time sequence from the first set of individuals to have their WES sequenced at CEGH-USP, batch 1, and batch 21 last.

During this period, some changes were made to the protocol, such as library preparation starting as Illumina Nextera and moving to Agilent's SureSelect capture.

Alignment to the reference human genome GRCh37/Hg19 was done with BWA-MEM (LI; DURBIN, 2009) with a minor modification from the human reference, excluding the sequences that did not have precise chromosome information and were classified as Unknown. The CEGH-USP bioinformatics team was responsible for sequencing and aligning the samples, and their scripts are not included in this study; nevertheless, the scripts developed by this study will be included in the attachments.

Each sample was genotyped separately using the Genome Analysis Toolkit Haplotype Caller (GATK - version 3.4) (MCKENNA et al., 2010). The decision for calling all genotypes in gVCF format was made to maintain the information about 
each base position and avoid the biased comparison of different VCF files, as described previously. After each calling, all the gVCF files were combined for a unique call with GenotypeGVCF. With the gVCF file it is possible to combine all individuals in one VCF and maintain each base information minimizing the effect of the sequencing batches. This effect can be seen by the approximation of batches 19 to 21 in this new approach, although some differences could not be removed, as it can be seen by the isolation of exome 18 (Figure 11).
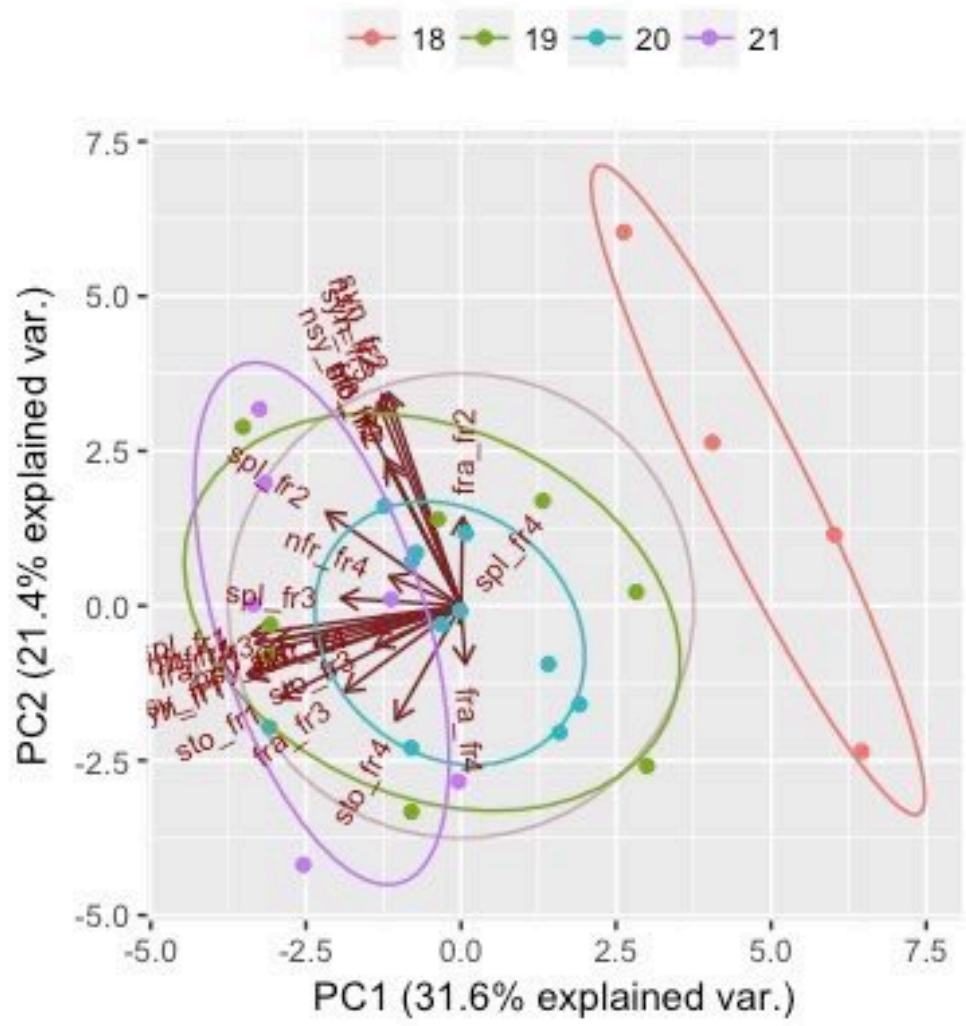

Figure 11: First two PC of the whole exome sequencing regarding only the coding variants for batches 18 to 21 .

Even with the remaining differences among WES batches, after the combination of the gVCF files, all the individuals were maintained for further analysis, in order to simulate the real clinical-laboratory context where most of the bioinformatics strategy were done to control for sequencing biases.

Since different gene lists are being tested, the VCF file was filtered to contain only the information for those specific genes. The chromosome coordinates (BED) files for each gene list was obtained by UCSC Table Browser, by taking GRCh37/Hg19 as genome reference and whole genes as parameters. The resulting variant call format (VCF) file, with all 168 individuals, was filtered based on the BED file for each gene list and converted for annotation with ANNOVAR (WANG; LI; HAKONARSON, 2010), and other customized annotations developed by the CEGH-USP bioinformatics group. Variants with genotype-likelihood below 98 score, according to GATK, with less than 10x of depth and alternative balanced alleles below 0.3 were removed (AZIZ et al., 2015; MATTHIJS et al., 2015; PIROOZNIA et al., 2014). 
A script in R (TEAM, 2017) was developed from this annotated file to count the number of variants in each class for each individual. The counting was split into two different tiers, one based on population frequency and the other based of protein alteration. The first took into consideration three population databases, the 1000Genomes (http://www.internationalgenome.org), 6500 exomes (evs.gs.washington.edu/EVS) and ABraOM (http://abraom.ib.usp.br), the variants were classified as singleton (appear in only one individual from the 168 probands, with no frequency in the databases), rare (below 0.01 in all databases), polymorphism (between 0.01 and 0.05 in at least one database), and common (above 0.05 in at least one database). The protein alterations were classified as frameshift, stop (stopgain and stoploss), splicing (only two bases adjacent to the exon-intron junction), nonframeshift, missense, synonymous, regions of micro RNA, non-coding RNA, regulatory sites, binding sites, 3'-UTR and 5'-UTR.

All the command lines from calling to the final counting are shown on Attachment 22.

\subsection{Comparisons pipeline}

A script in $\mathrm{R}$ was developed to analyze different approaches to compare affected individuals with control individuals based on the quantity of variants for each class, as described in the previous session. An example of the counting file is given on Attachment 23.

This report in $\mathrm{R}$ is a compilation of different common strategies for analyzing whole exome sequencing (WES) with four multivariate models. The report starts with an exploratory analysis of the total variants, first, with the distribution of the common synonymous variants (frequency above 0.05) to address possible outliers in the sample, using the rational that most common synonymous variants have a neutral pattern of selection and are uniformly distributed in the populations worldwide, expecting similar numbers of variants in this category for all individuals. Second, with a histogram of all the variants, to observe the different distribution pattern for each variant and incorporate this information to the analysis. Also, it allows the observation whether a particular variant had a different pattern from that which was expected, thus indicating the presence of possible errors in the process.

The next step evaluates whether the whole sample can be sorted into different clusters. Since genetic evidence does not see ASD as a single disease but as a number of etiologically distinct conditions (LA TORRE-UBIETA et al., 2016), or both extremes of a normal continuum (BOURGERON, 2015), in addition to the factors contributing to differences in the sequencing process and the particularities of each individual, it is prudent to group them by using a clustering method, so that more homogeneous sets for the final comparison are obtained.

A non-hierarchical method (K-means) was chosen since it is less susceptible to 
atypical samples, to the distance measures and to the possibility of irrelevant variables (HAIR et al., 2009). The method used was the Hartigan-Wong (HARTIGAN; WONG, 1979). The seeds were chosen at random based on the initial number of clusters and were repeated 50 times each. In the case of two clusters, for example, the k-means were calculated 50 times based on two initial individuals. We understood the limitations of not providing a matrix of centers for the initial seeding process, and we chose to continue the analysis in this format for two reasons: first, we considered 50 random choices of two pairs to be sufficient to represent the possibility for the best result, since it would involve almost two-thirds of the whole sample. This could randomly generate a better result than that of starting with a preconceived tree from other methods. The second reason was because with the $R$ package stats (TEAM, 2017), which includes the K-means function, used in this work, there was the possibility of no point being closer to one or more centers and result in error. The ideal number of clusters was chosen between one and 15, comparing the total within-clusters sum of squares. The last point with a high difference from the previous (the inflection point) was considered the best option. For the purpose of the analysis conducted in this study, all the reports had two clusters as the best choice.

The results obtained from the different clusters could be interpreted in three different ways. First, the findings present in the whole sample and confirmed in the clusters can be considered a robust general behavior of the disease under investigation. Second, the findings present in only one cluster can be the result of a specific relation that is evident in more homogenous groups and will require further investigations. Third, characteristics that are present in the whole sample but not confirmed in the clusters can be interpreted as a general characteristic that requires a higher quantity of samples, or as bias resulting from the presence of heterogeneous grouping and representations of affected and control groups alike.

After determining the cluster, in order to access possible deviations in sequencing and annotation, a correlation matrix of all variants was calculated. For coding regions, a smaller number of disruptive mutations than that of the variants considered neutral was expected, therefore we expect a smaller number of frameshift mutations than that of synonymous mutations. Following this same line of thought, we expect the number of common mutations to be higher than that of rare mutations within the same type of variant (LEK et al., 2016).

Despite these frequency properties, the mutations occur randomly and different individuals should have similar amounts of each mutation type; therefore, significant correlations between different types of variants are not expected. Thus, deviations from this pattern can be interpreted as characteristic of sequencing and annotation workflow and should be taken into account for future interpretations. Nevertheless, if there is difference between the correlation matrix of affected and control individuals, it could be possible that, for complex diseases, certain types of mutations may occur jointly, which can lead to this deviation. All the correlations were calculated with the R package psych (REVELLE, 2017), corr.test function, with Pearson correlation test as the correlation method, the graphical display was done with the corrplot package (WEI; SIMKO, 2017). The p-value matrix shows on the lower diagonal the $\mathrm{p}$-values for the correlations, and the upper diagonal shows the p-value 
corrected for multiple tests with the holm method, which is regarded as being less conservative than normally applied tests, such as the Bonferroni test (TEAM, 2017).

After the initial exploratory analysis of the variants to determine degrees of possible bias in the sample, the first attempt at identifying a possible mechanism to discriminate between the groups of interest was to investigate whether a particular variant can have a major effect on the affected group. All comparisons were done with the Wilcoxon rank sum test, regarded as being the same as the Mann-Whitney test (FIELD, 2017), with the R package stats, wilcox.test function. This test is the equivalent to the Student's t-test for non-parametric samples and is specific for comparing two groups using ordinal arrangements. This ordinal grouping can be applied to the ASD model because of the additive effect expected; for example, one is different from two and could be considered one degree below; if one was for male and two for female, this same ordinal relation would not apply. The same approach was performed for the clusters to observe whether some of the previous findings could be attributed to a deviation in the number of affected individuals within each cluster. The graphical display was performed with the ggplot2 package from R (WICKHAM, 2009).

The investigation continues with more elaborated questions about the possible models that could explain and discriminate between affected and control individuals. There are three main approaches that will be described in further detail, one with lossof-function and missense mutations, one with Multivariate Models, and a last one with Principal Component Analysis.

\subsubsection{Rare Loss-of-function and missense}

Previous studies found a higher number of rare disruptive mutations (LoF and missense) in individuals with autism spectrum disorders (ASD) than in control individuals (DEVLIN; SCHERER, 2012; IOSSIFOV et al., 2012; KRUMM et al., 2015; STEIN; PARIKSHAK; GESCHWIND, 2013). According to these findings, the first approach to the whole exome sequencing analysis in the clinical laboratory is to look for likely gene-disruptive variants and report them as related to the clinical phenotype. Thus, the amount and severity of this type of variants were compared between the ASD and control groups.

All loss-of-function (LoF) mutations (frameshift, canonical splicing sites and stopgain/loss) were summed into one category of LoF mutations and further separated by population frequency, and rare and singleton variants were tested separately. A contingency table was calculated in three different manners, in order to test different categorization approaches, instead of sorting the individuals into two categories of presence and absence of LoF mutations. First the individuals were sorted out based on frequency, with the attempt to have the same number of individuals in each category; second, they were sorted into presence of any number of mutations versus absence, and third, the groups were sorted out by a non-hierarchical clustering method. This was done separately for rare and singleton variants, by applying the 
Pearson $X_{2}$ test (PX2), the best choice for unconditional trials (LUDBROOK, 2008). Only the p-values below 0.05 were taken into account for further discussion. Since the contingency table is limited to grouping the individuals into two categories, in order to test for each number of LoF in particular, a receiver operating characteristic (ROC) curve was plotted for each number of mutations.

The ROC curve is a graphical approach to selecting classifiers based on their performance (FAWCETT, 2006). It is constructed based on the proportion between true positives (TP) (positives correctly classified as positives divided by the total number of positives) and false positives (FP) (negatives incorrectly classified as positives divided by the total number of negatives) (FAWCETT, 2006). Each quantity of LoF was regarded as a threshold to classify the individuals, a contingency table was calculated for each quantity and the results of TP and FP were plotted, where the more a point deviates from the 45 degrees line the more this particular point is capable of discriminating the groups (SWETS; DAWES; MONAHAN, 2000).

The missense approach was different, because of the high number of nonsynonymous variants in each subject and the resulting need to discriminate the mutations with higher probability of disrupting the protein function. Also, missense mutations have a highly specific pattern and tend to hit residues that are extremely close to each other and in the same domain, which occurs in Wnt and synapse pathways (LUO et al., 2018) in particular. Variant prioritization was conducted with the software SPRING (Snv PRioritization via the INtegration of Genomic data) (WU; LI; JIANG, 2014). This software is "based on six functional effect scores calculated by the existing methods (SIFT, PolyPhen2, LRT, MutationTaster, GERP and PhyloP) and five association scores derived from a variety of genomic data sources (gene ontology, protein-protein interactions, protein sequences, protein domain annotations and gene pathway annotations)" (WU; LI; JIANG, 2014). The software is valid for diseases whose genetic bases are poorly known and was tested for the capability to detect pathogenic de novo mutations in ASD, using 34 gene seeds to prioritize the query.

For this study, the variants of interest were separated from the annotated VCF file, already filtered for the gene list. For seeding genes, we used the same list to test whether affected and control individuals would present different distribution patterns just with the damaging predictions from the software. After the SPRING prioritization, all the variants received three numbers: the first referring to the rank for their strength of association with the query disease, the second referring to the pvalue, which is a statistical significance that the variant is causative for the disease, and the third referring to the q-value, which is the converted p-values for addressing the multiple test correction problem.

The missense variants were split into two groups, rare and singleton variants, then each one of these groups were evaluated by the SPRING software to rank all the variants based on the specific gene list and pathogenicity, and only variants raking one were used for further analysis. The q-values were log transformed and the samples were compared in two different patterns. First, the distribution of the number of variants for each $\log$ (q-value) for affected and control individuals, and second, the average of each $\log (\mathrm{q}$-value) per individual, also separating affected individuals from 
control individuals. It is expected that if there is an enrichment of likely genedisruptive mutations in the ASD group, it would show as a deviation toward the higher $\log (q-v a l u e)$ for the ASD group, in both comparisons.

Both approaches, with LoF and missense variants, can be regarded as proxy for interpreting the whole exome sequencing in the laboratory routine. The analysis focus on the individual, searching for the most disruptive rare mutations and associating them with genes that were previously described as related to ASD.

\subsubsection{Discrimination models}

Four multivariate discrimination methods were chosen to initially test the difference between the groups of interest, (a) logistic regression, (b) decision tree, (c) neural network and $(\mathrm{d})$ support vector machine. These methods were chosen because they are adequate for binary data and use machine learning techniques at different levels of depth.

\subsubsection{Classification Performance}

All the multivariate approaches applied in this work will provide a discriminate model, which stress the importance of evaluating its power to discriminate between the groups of interest. The following evaluating approaches were chosen in this study: the area under the ROC curve (AUC), the KolmogorovSmirnov (KS), and the Hand metric $(\mathrm{H})$. All the metrics were obtained with the HMeasure function from the hmeasure package in $\mathrm{R}$ (ANAGNOSTOPOULOS; HAND, 2012).

Situations in which the discriminant model returns the probability of the event, it is possible to construct two normal distributions for true positive and false positive rates, plotting on the $x$ axis the probability estimated by the model for each individual (SWETS; DAWES; MONAHAN, 2000). If each probability point, the $x$ axis determined above, is plotted on a chart where the $\mathrm{x}$ axis is the rate of false positive and the $y$ axis is the rate of true positives, the union of all the points for each probability is the ROC curve for that model. The AUC is the area under this curve, and it measures how much the model classifier is superior to a classifier chosen at random (FAWCETT, 2006). The higher the AUC the more accurate the group-discrimination model; the value to consider a good model will be attributed depending on the specific problem and the threshold is commonly established based on a previous model for the same individuals (FAWCETT, 2006). The reports establish the threshold at 0.8 since a deviation in the initial number of individuals from control and ASD groups would bring an initial discrimination, chance model at about 0.66 (two-thirds of the samples in this study are control individuals). 
The KS is a non-parametric, statistic test developed to find out whether the population has a specific distribution, since it compares the distribution seen in the population with a hypothetical/ known distribution (FIELD, 2017). A modification to the original use can be done to compare two distributions, allowing the test to be used as a measure of goodness-of-fit for predictive models (QIN, 1997). There is no stablished threshold for this metric, since it is a comparison metric, and, unlike the AUC that starts at 0.5 , which is equivalent to a random choice, this metric starts from zero, thus we established the threshold at 0.4 for the purpose of this study.

H-measure was described in 2009 by David J. Hand as an alternative to the previous metrics, such as AUC and KS, mainly because it considers different penalties for misclassifying classes 0 or 1 (HAND, 2009). This is particularly important if we are dealing with a test that has different consequences on each group. For the purpose of this study, we set the worst misclassification to losing a true positive case, in other words, the misclassification as false negative was more problematic. This decision was made because most individuals that come for a genetic test already have a clinical ASD diagnosis, therefore the probability of this individual being a true ASD case is higher. In turn, individuals that come for different diagnosis cannot exclude ASD, which increases the probability of having an underlying ASD diagnosis. For this reason the parameter severity.ratio is set to 0.75 . The same threshold of 0.4 as KS was established.

The number of individuals classified as false positive, false negative, true positive and true negative is calculated for each prediction model.

\subsubsection{Logistic Regression}

From all the regression models, logistic regression was the method chosen for this study because the variable response, considered here as affected or not affected, follows the Bernoulli distribution of zero and one (BARRETO, 2011). The logistic regression will construct a predictive model with the final result being log odds, which can be interpreted as the probability of the response variable designated as one, in this case, affected. Logistic regression is not as sensitive to scale and distribute the original independent variables as the linear models are (BARRETO, 2011), allowing the data to be used in their original format with no further manipulation. For the logistic regression, the glm function from the package stats in $\mathrm{R}$ was used. This function calculates the generalized linear models and can be applied to many different models, and to specify that it concerns a logistic regression the parameter family=binomial(link='logit') is set in the formulae.

The samples were split into two groups: half of the individuals for the learning group and half of them for the test group, by using a random sampling that followed the original proportions of the ASD and control individuals, with function createDataPartion from the package caret in $\mathrm{R}\left\{\mathrm{Kuhn}: \mathrm{C} 8 \_\mathrm{eJz} \_\mathrm{I}\right\}$. From this first model a backward approach was applied to select the variables for the best model with the function step from the package stats in R. The backward approach consists of the 
removal of the variable with less effect and the recalculation of the logistic regression and the Akaike Information Criteria (AIC). The AIC measures the goodness-of-fit and simplicity of the models by using the concept of entropy, when comparing between two different models, where the one with the smaller number of AIC will be a better model (AKAIKE, 1985). The step function tests all models until it finds the one with the smallest AIC, which will be interpreted as the best model. This final model was applied to the test sample and, from this result, we obtained the ROC curve and the discrimination metrics, AUC, H and KS. The true positive, false positive, true negative and false negative results were calculated with the best discriminant threshold found.

\subsubsection{Decision Tree}

The method of classification and regression trees (CART) was described in 1984 by Breiman et al. as an improvement to the current tree methods, and an alternative to linear regression models (BREIMAN, 2017). The CART is a binary recursive partitioning procedure that handles data in their raw form. Beginning in the root node, with all the individuals, the data are split in two children with lower parent node's entropy, and this process continues until a stop rule is applied, or they can grow to a maximum size and then be pruned back (HASTIE; TIBSHIRANI; FRIEDMAN, 2013; WU et al., 2007).

In this study, we applied the classification tree, which was allowed to grow to the maximum size, and then we applied cross-validation with the test sample. The individuals were split into learning and test groups, as described for the logistic regression. The variables used to construct the tree and the information about each split were printed. From this testing result, we obtained the fitting of the model, thus making it possible to select the best tree for that dataset. The error columns have been scaled so that the first node has an error 1.

The best model is the one with the minor error rate that avoids overfitting (HASTIE; TIBSHIRANI; FRIEDMAN, 2013), and, for this reason, pruning the tree could be necessary to obtain the best possible model without overfitting. The xerror is an important metric that supports the pruning decision. The xerror is a summary measure of the fit of the model to the test sample, and the better the fit the smaller the xerror. The pruning can follow the rule of stopping in the split which has the small improvements from the anterior. All the decision tree calculations were done with the rpart package (THERNEAU; ATKINSON; RIPLEY, 2017).

\subsubsection{Neural network}

The neural network is a nonlinear statistical model that consists of a two-stage regression or classification model, typically presented by a network diagram 
(HASTIE; TIBSHIRANI; FRIEDMAN, 2013). The neural network is a common approach to machine learning, with many modifications to improve specific questions (ANASTASIADIS; MAGOULAS; VRAHATIS, 2005; RIEDMILLER; (null); 1993, 1993). The one applied in this study is a resilient backpropagation with weight backtracking (RIEDMILLER; (null); 1993, 1993). The backpropagation is the most widely used algorithm for supervised learning and consists of the repeated application of a specific rule to compute the influence of each coefficient on the network regarding an arbitrary error function (RIEDMILLER; (null); 1993, 1993). The resilient backpropagation performs a direct adaptation of the weight step (which determines the coefficients) based on local gradient information. This modification reduces the number of learning steps with less computational effort (RIEDMILLER; (null); 1993, 1993).

Neural network computations were conducted with the neuralnet package in R (FRITSCH; GUENTHER, 2016). For better results, the original dataset was scaled from 0 to 1 , and two hidden layers were chosen with 15 and 5 neurons, respectively. The final result is applied to a test sample (in the same mode as already described) to compute the model's goodness-of-fit.

\subsubsection{Support Vector Machine}

The support vector machine is a machine learning approach that searches for the optimal hyperplane to separate the groups of interest. The difference between support vector machine and other linear boundary classifications is that it produces nonlinear boundaries by constructing a linear boundary in a large, transformed version of the initial space (HASTIE; TIBSHIRANI; FRIEDMAN, 2013). Each data is plotted in an $n$-dimensional space (being $n$ the number of variables computed). The support vector machine will construct hyperplanes that best separate the groups not restricted to three dimensions.

The choices of the hyperplane are very similar to that of the decision tree process. The support vectors have to separate the samples in groups with minimal intersection, which is a particular challenge to avoid overfitting (HASTIE; TIBSHIRANI; FRIEDMAN, 2013). All these computations were done with the e1071 R package (MEYER et al., 2017). We applied the linear kernel because we did not have previous information on what would be the best method to compute the hyperspace. So, we selected the classification approach using support vector machine to determine the probabilities of each classification and plot the probability associated with the target. The model was applied to a testing sample to determine the discriminant metrics.

\subsubsection{Principal Component Analysis}


The principal component analysis is a multivariate approach that reduces the variance of the samples from a correlation or covariance matrix to vectors of complex variables that are related to the information of more than one variable of the original dimension. In summary, the information within one principal component aggregates that of more than one variable regarding the correlation or covariance between them (MINGOTI, 2005).

For this study two approaches were used: the first with the counting matrix and the second with the raw VCF file. Principal components were determined for the counting matrix with the prcomp function in $\mathrm{R}$ stats package, and, for this purpose, the covariance matrix with all variables shifted to be zero centered and scaled to a unit variance. These modifications had to be done due to the largely different numbers of variants in each class.

The raw VCF approach was conducted with the function snpgdsPCA from the R package SNPRelate (ZHENG et al., 2012). This package was created to accelerate the computations on SNP data for principal component analysis and identity-by-descent measures to decrease the computational time of the GWAS studies. To compute the principal components, they construct the covariance matrix from all variants in the raw VCF file, mainly aiming at finding and correcting population structure that could interfere with the GWAS analysis. This functionality was co-opted for this study and the population structure investigated was the difference between ASD and control groups.

The R script used for developing the report with all the analysis above is on Attachment 24. 


\section{Results}

All the results described in this chapter will be related to the files created with the scripts detailed in Material \& Methods, and, because of the large number of pages each report generated, they are not being printed with the thesis but can be accessed through the link below.

\section{https:// www.dropbox.com/sh/cqyhdrygfhoys3b/AADXrpaMS emgn WyL-17mnSFa?dl=0}

The name of the respective file will be the same as that of each chapter. For example, the file related to the exploratory analysis will be: exploratory-analysis.html (Figure 12). All the files are in html extension, which can be open in any device, desktop or mobile, after download.

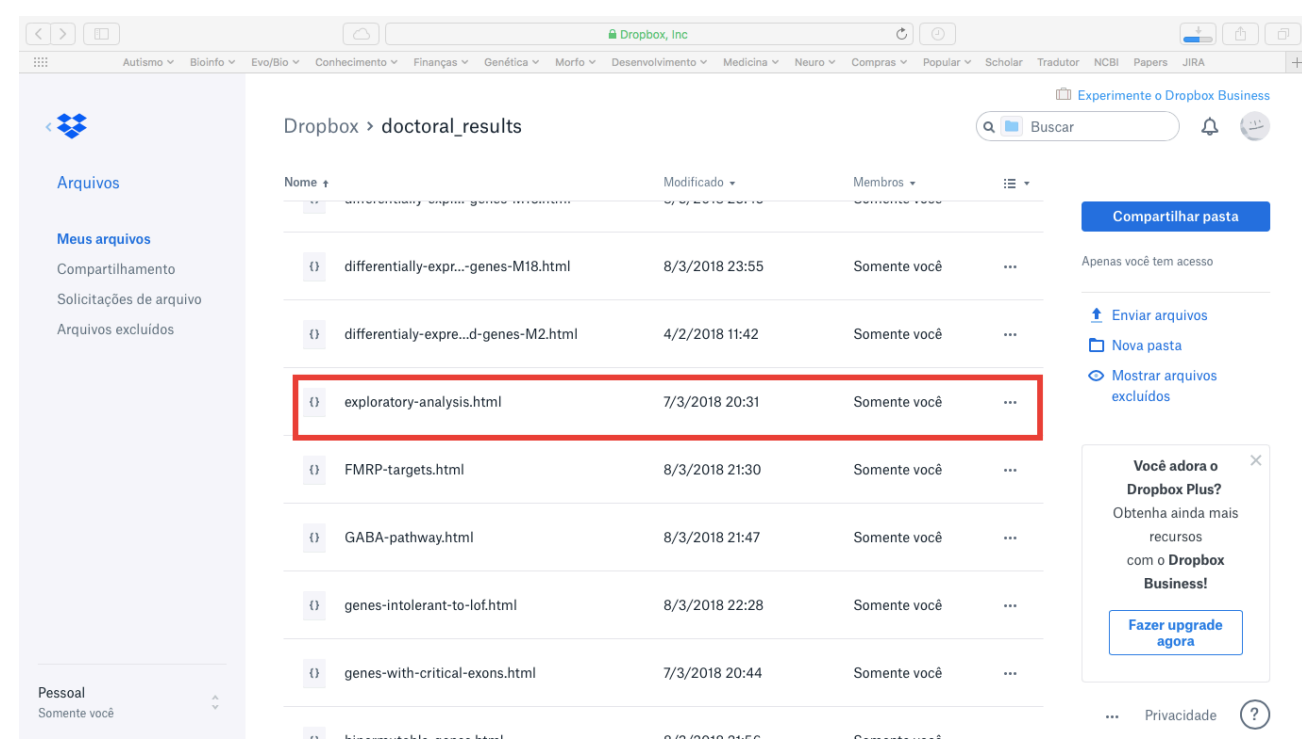

Figure 12: Visualization of the archives in the reports folder, the report inside the red box is the exploratory-analysis.html.

\subsection{Exploratory Analysis}

This description concerns the first report prepared with whole exome sequencing and no gene filtering (Figure 13). This is an example of the steps taken for interpreting the full report. For the subsequent reports concerning specific gene filtering, only the results that are significant at a level of p-value below 0.05 (marked in red in the report - Figure 14), or, for the multivariate models, three discriminant metrics, H, AUC and $\mathrm{K}$, above the thresholds: $\mathrm{H}>0.4$, AUC $>0.8$ and $\mathrm{K}>0.4$ (marked in blue in the report - Figure 15) will be discussed. The main component analysis will be described if there is a notable difference between the dispersion of the groups after 
removing the outliers.

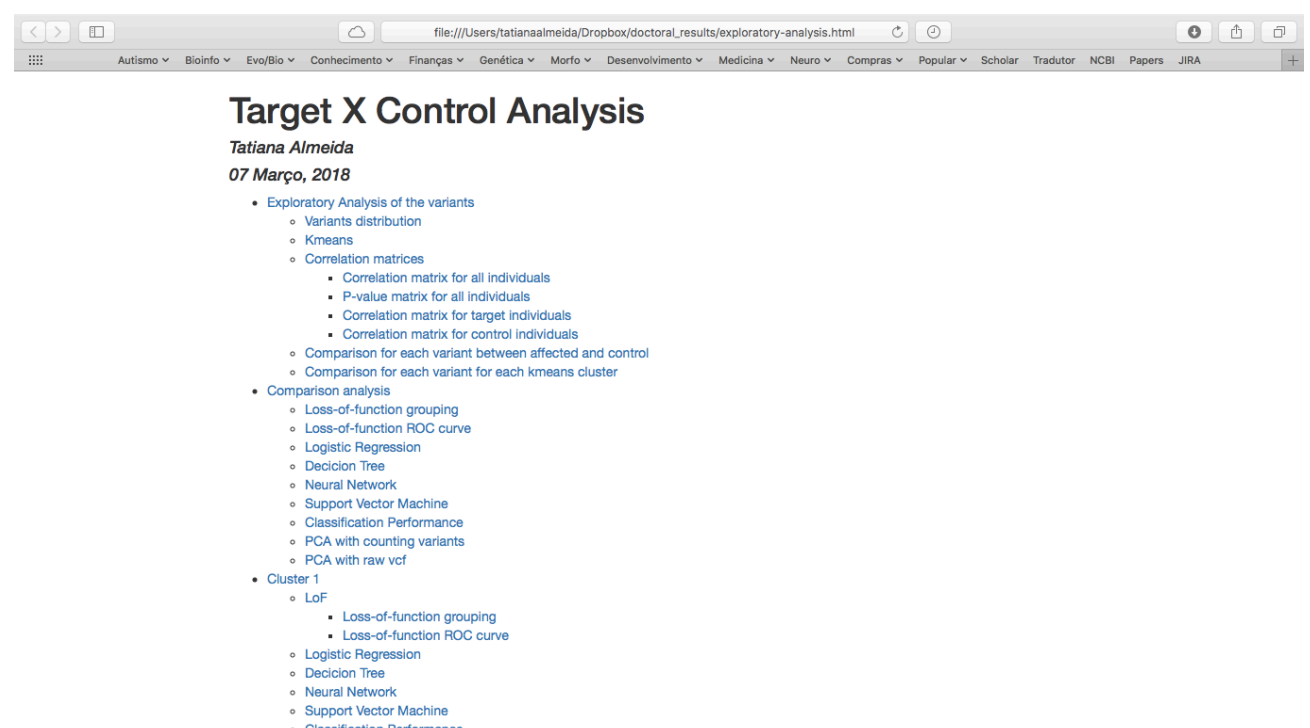

Figure 13: Screenshot of the index of the exploratory analysis.html report.

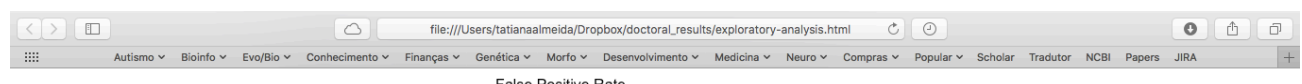

False Positive Rate

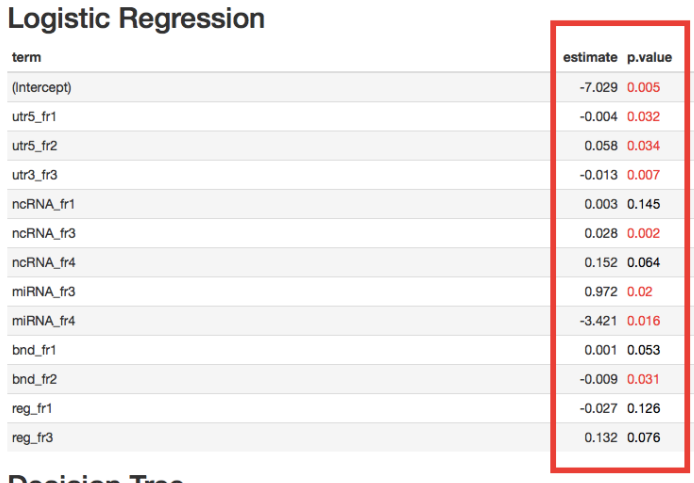

Decicion Tree

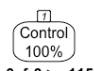

Figure 14: Screenshot of an example of p-values bellow 0.05 on the report. 


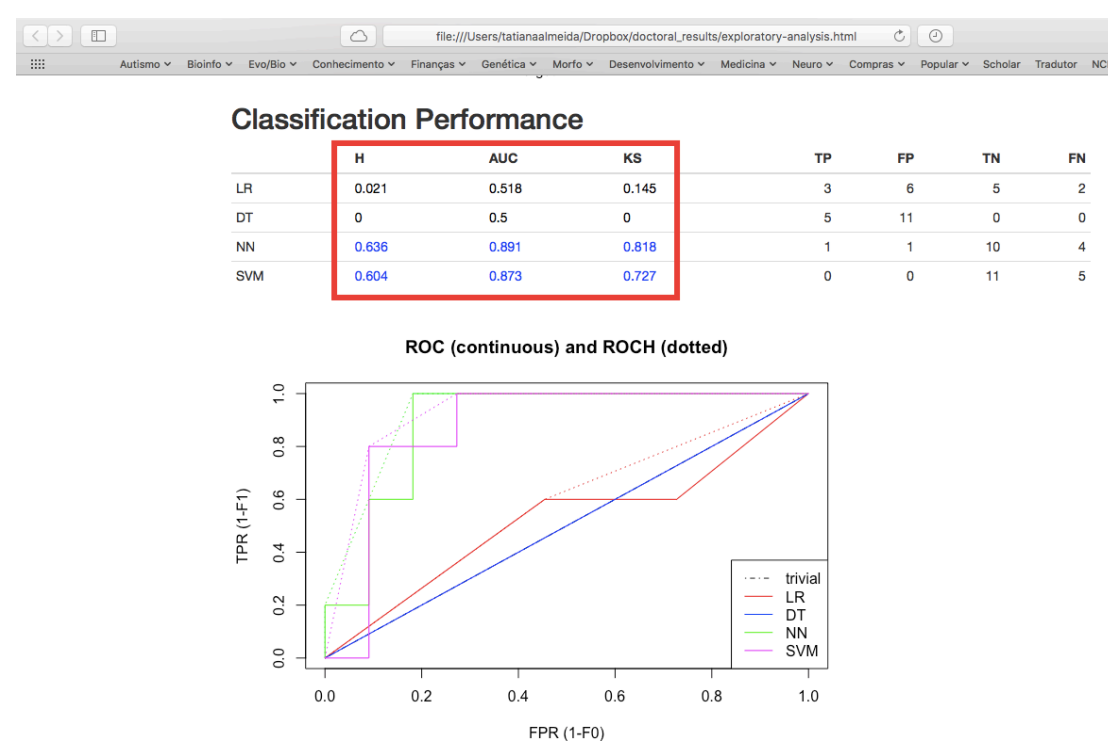

Figure 15: Screenshot of an example of $\mathrm{H}$ and KS above 0.4 and AUC above 0.8 on the report.

Due to the number of names of the variants' classes, the abbreviation was conducted in two parts, separated by underline. The first part is the name of the variant and the second one is the population frequency, as follows:

\begin{tabular}{|l|l|}
\hline Type of variants & \\
\hline fra & Frameshift \\
\hline sto & Stopgain/loss \\
\hline spl & Canonical splicing $(+1,+2,-1,-2)$ \\
\hline nfr & Nonframeshift \\
\hline nsy & Nonsynonymous \\
\hline syn & Synonymous \\
\hline utr3 & $3^{\prime}$-UTR \\
\hline utr5 & $5^{\prime}$-UTR \\
\hline ncRNA & Noncoding RNA \\
\hline miRNA & Micro RNA \\
\hline bnd & Transcription binding sites \\
\hline reg & Regulatory sites \\
\hline lof & Frameshift+Splicing+Stopgain $/ 1$ \\
\hline Frequencies & oss \\
\hline fr1 & \\
\hline fr2 & Above 0.05 \\
\hline fr3 & Between 0.05 and 0.01 \\
\hline fr4 & Below 0.01 \\
\hline
\end{tabular}

As an example, if we refer to a frameshift variant present in only one individual and absent in population data (singleton), the abbreviation will be fra_fr4; if we refer 
to a regulatory site with common frequency above 0.05 , it will be reg_fr 1 . This table will be printed on the first page of attachments 25 .

\subsubsection{Removing outliers}

The synonymous distribution chart shows that, despite the non-homogeneous composition of the samples, there are no evident outliers, and, therefore, all the 168 individuals were maintained in the analysis.

\subsubsection{Variants distribution}

This analysis shows that the frameshift, stopgain/loss and nonframeshift distributions have a normal distribution (Shapiro-Wilk $p=0.98,0.35,0.10$, respectively). However, the splicing, nonsynonymous, synonymous, 3'-UTR, 5'-UTR, binding sites, miRNA, ncRNA and regulatory sites, contrary to what would be expected, do not have a normal distribution (Shapiro-Wilk $\mathrm{p}=0.03,1.05 \mathrm{e}-07,1.6 \mathrm{e}-07$, 6.6e-16, 7.5e-11, 9.9e-05, 3.6e-07, 1.7e-13, 0.0001, respectively). The histogram on the report reveals a tendency to a bimodal distribution for these variants (Figure 16).

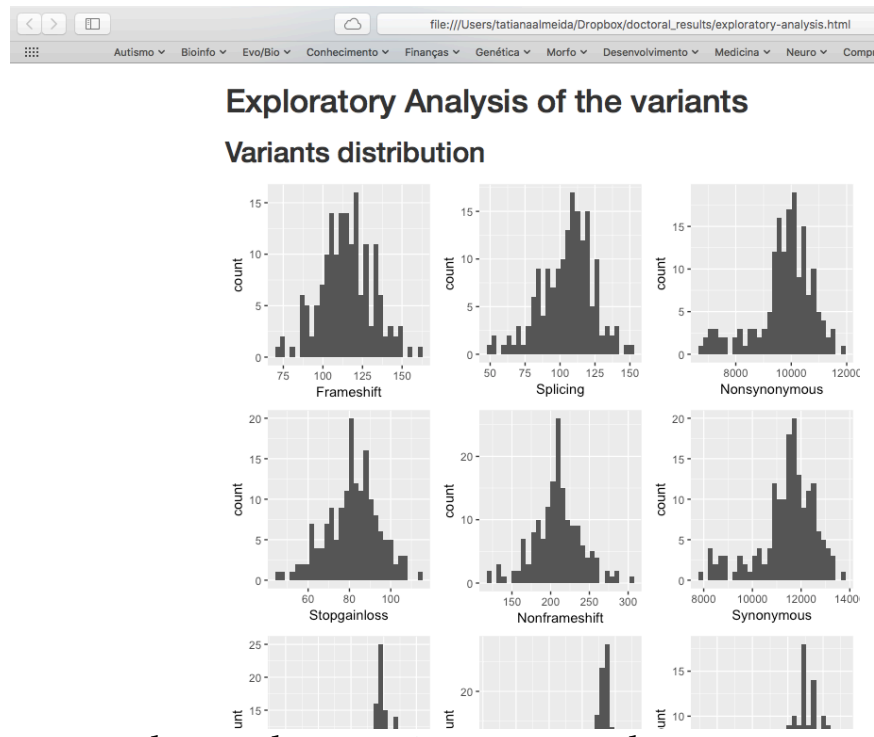

Figure 16: Screenshot of the distribution of each type of variant for all the individuals on the report.

One hypothesis for this bimodal distribution is the difference among the capture strategy and the alignment pipelines along the years. Figure 17 shows the distribution of the quantity of common nonsynonymous and synonymous variants across different WES batches and there is no clear distinction between the number of variants and the period within which WES was performed. The same occurred for the splicing, binding sites and regulatory sites. Therefore, the non-normal distribution for 
these variants cannot be attributed to the capturing and aligning differences occurring over the years.

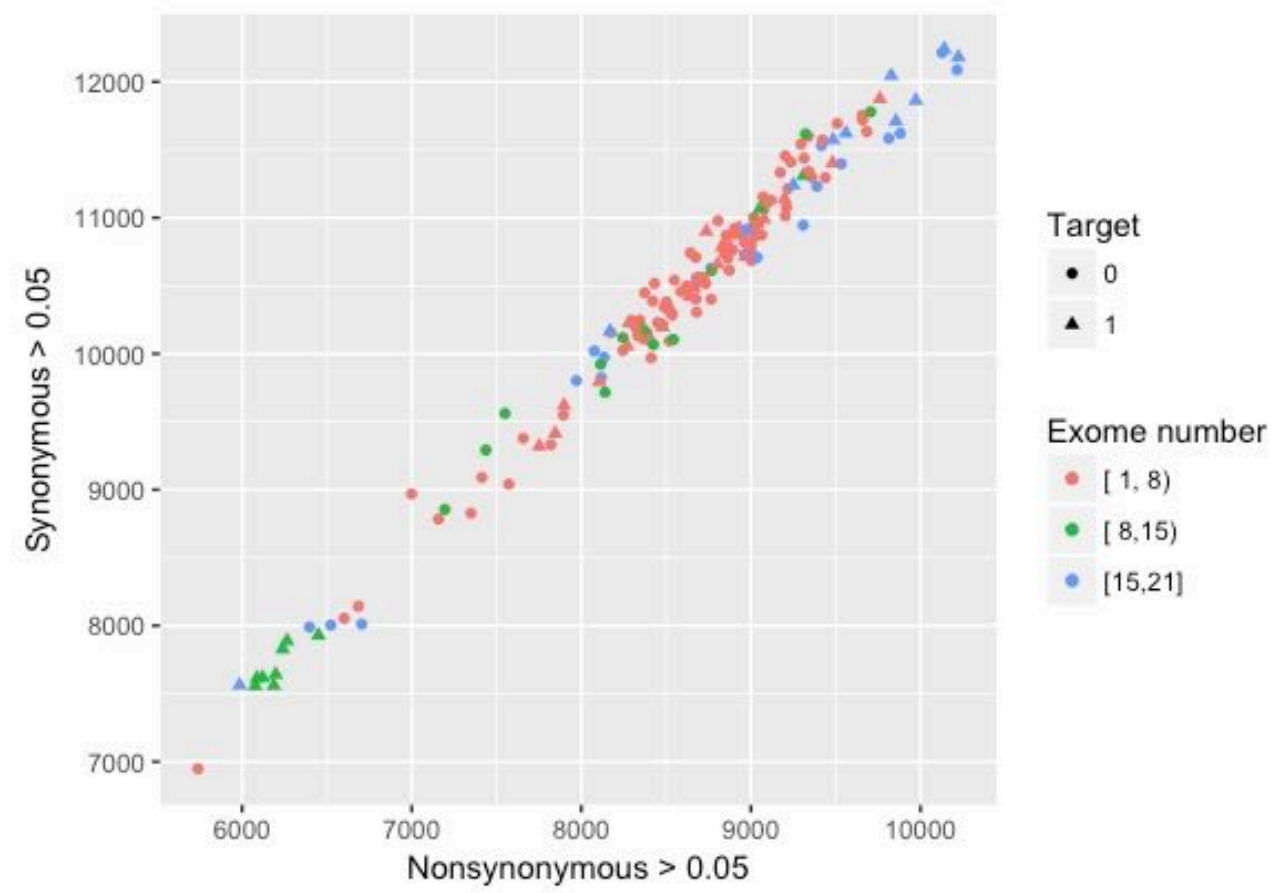

Figure 17: Synonymous and nonsynonymous distribution of the common variants for each individual separated by the number of the WES batch.

However, for the non-coding variants $5^{\prime}$-UTR and $3^{\prime}$-UTR, the distribution has a clear difference explained by the different WES batches (Figure 18), this pattern is the same for miRNA and ncRNA. The batches above number 14, which were most recently sequenced, showed less regulatory variants. 


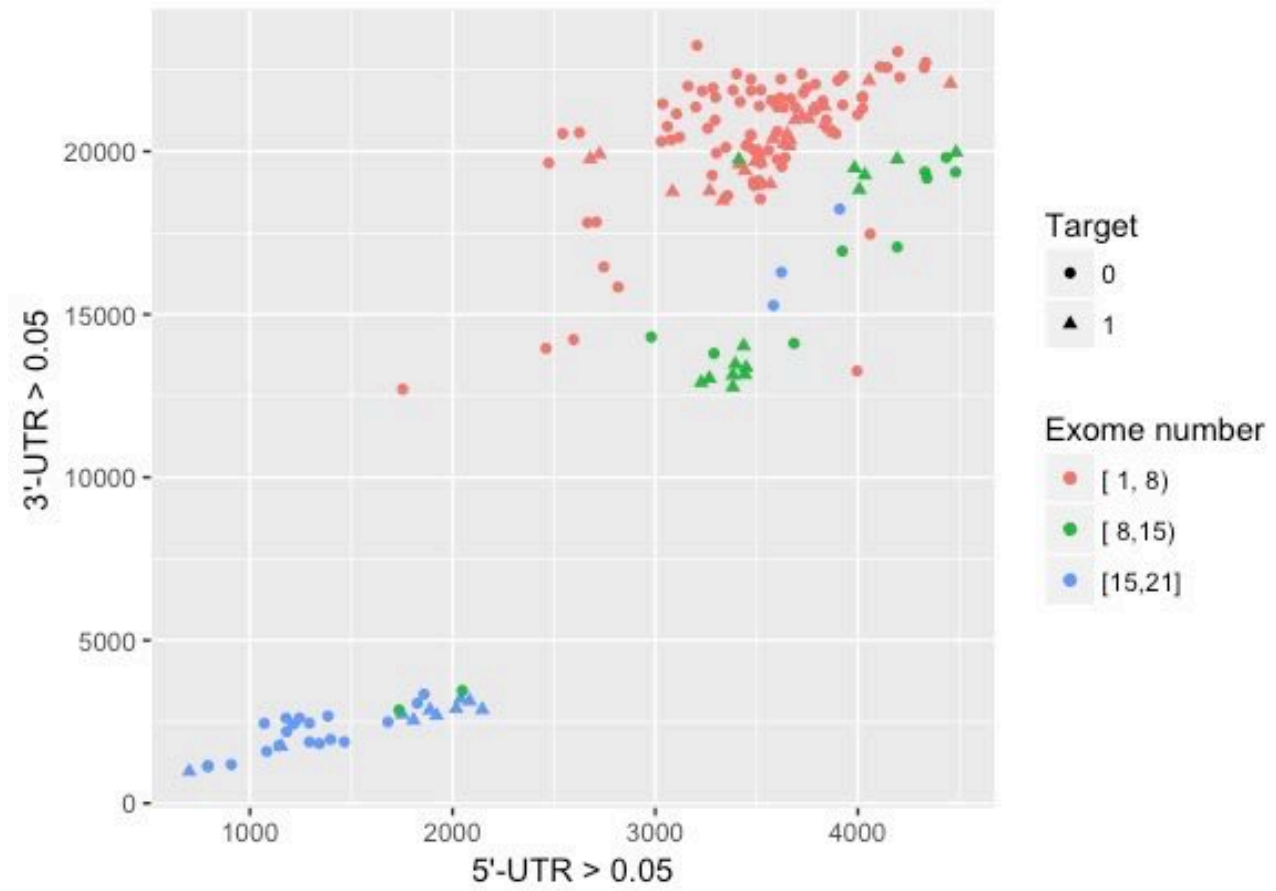

Figure 18: 3'-UTR and 5'-UTR distribution of the common variants for each individual separated by the number of the WES number.

These charts show that capture and alignment strategies can have a strong effect on the analysis of non-coding variants in particular. As a result of these differences, the results concerning these regions will not be further analyzed. However, since the pipelines were constructed to be applied to any scenario, including different diseases, they were kept on the reports.

\subsubsection{Kmeans}

The screeplot shows the inflection for the optimal number of clusters. Two clusters were chosen here and used in all the subsequent analyses, since they are the points in which there is still a major difference in the sum-of-square comparison among three clusters (Figure 19). 


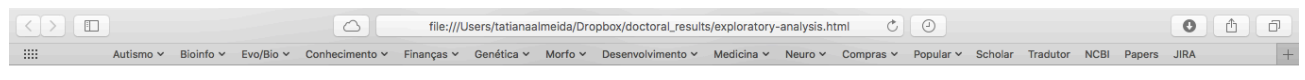

Kmeans

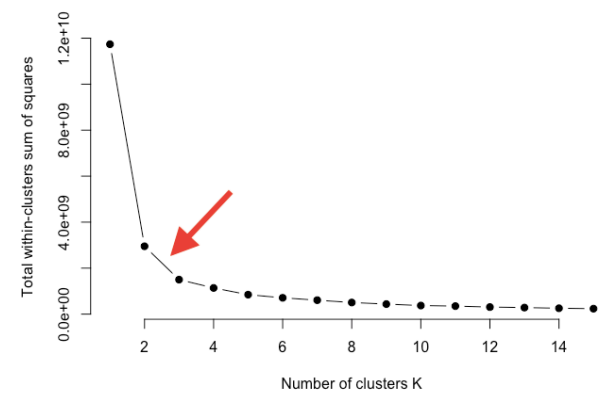

Correlation matrices

Correlation matrix for all individuals

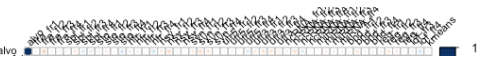

Figure 19: Screenshot of the screeplot for the kmeans sum-of-squares, with the arrow marking the midpoint between the two points with most inflection.

The best explanation for this division, as shown in Figure 20, is the number of 3'-UTR and 5'-UTR variants for each subject, which can be a proxy for WES capture and alignment strategy, as discussed above, strongly because most of the individuals of the second cluster are present in more recent WES batches.

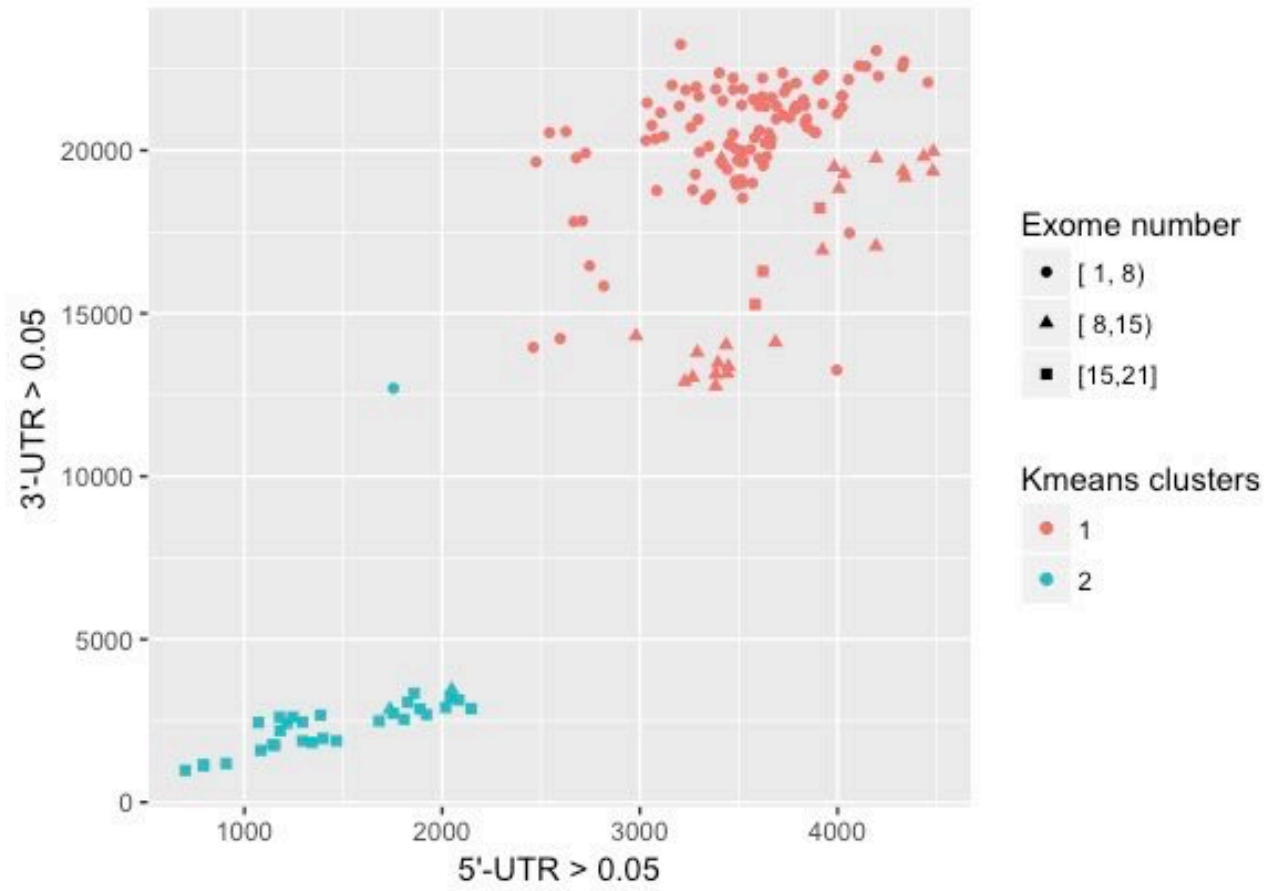

Figure 20: 3'-UTR and 5'-UTR distribution by Kmeans cluster and WES number. 


\subsubsection{Correlation Matrices}

The Correlation matrix for all the individuals shows the pairwise correlations among all the variants (Figure 21), and P-value matrix for all the individuals shows on the lower diagonal the $\mathrm{p}$-value for all the correlations and the $\mathrm{p}$-value corrected for multiple tests is shown on the upper diagonal (Figure 22).

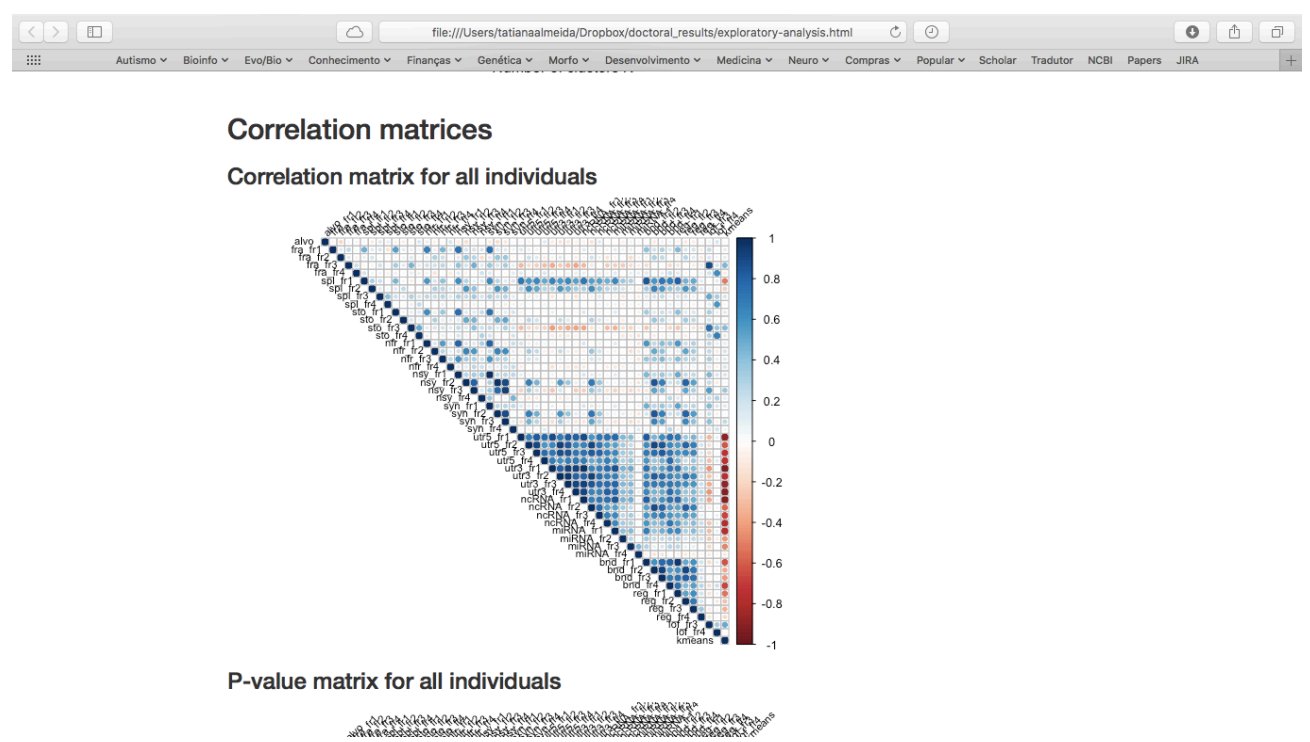

Figure 21: Screenshot of the plot for the correlation among the variants, the lighter the color, the closer to zero (no correlation), blue represents a positive correlation and red represents a negative correlation.

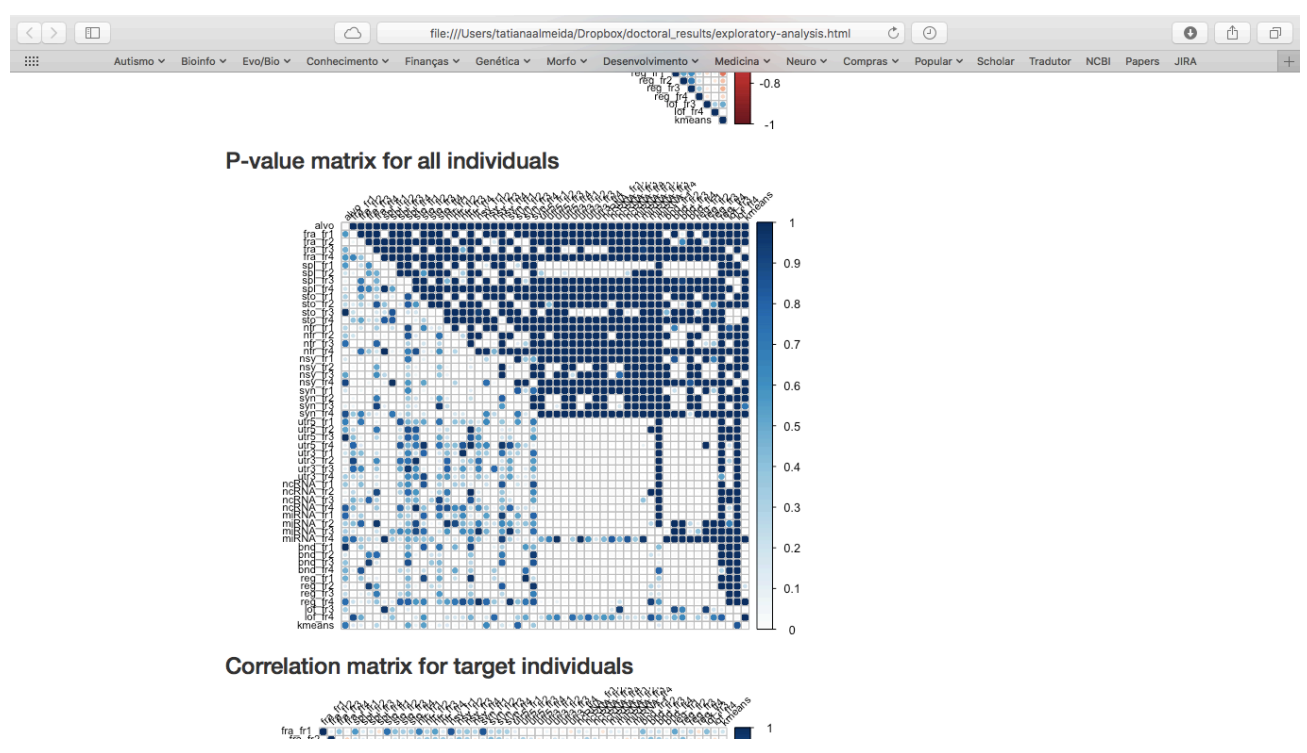

Figure 22: Screenshot of the plot for the p-values associated with the correlations above, the upper diagonal shows the $\mathrm{p}$-values corrected for multiple tests and the darker blue shows the values that are closer to one. 
The most prominent correlations occur among splicing, 3'UTR, 5'UTR, ncRNA, miRNA and regulatory regions. Most of these correlations can be attributed to the capture and alignment differences that were discussed previously. Other correlations that draw attention and cannot be explained by the difference in alignment are seen on Table 1.

Table 1: Significant correlations not explained by WES data capture and alignment.

\begin{tabular}{|l|l|l|l|}
\hline & & Correlation & P-value \\
\hline Fra_fr2 & Nfr_fr2 & 0.30792824 & $5.160595 e-05$ \\
\hline & Nsy_fr2 & 0.37571758 & $5.632275 e-07$ \\
\hline & Syn_fr2 & 0.38959341 & $1.951014 \mathrm{e}-07$ \\
\hline & Sto_fr1 & 0.33166109 & $1.195594 \mathrm{e}-05$ \\
\hline & Sto_fr3 & 0.45095636 & $9.611695 \mathrm{e}-10$ \\
\hline & Nfr_fr3 & 0.26410295 & $5.631498 \mathrm{e}-04$ \\
\hline Sto_fr1 & Nfr_fr1 & 0.618509682 & $5.266167 \mathrm{e}-19$ \\
\hline & Nfr_fr3 & 0.374817077 & $6.023304 \mathrm{e}-07$ \\
\hline Sto_fr3 & Sto_fr4 & 0.50811621 & $2.392915 \mathrm{e}-12$ \\
\hline Sto_fr4 & Nsy_fr4 & 0.30196937 & $7.309479 \mathrm{e}-05$ \\
\hline Nfr_fr1 & Nfr_fr3 & 0.55806064 & $4.687232 \mathrm{e}-15$ \\
\hline & Syn_fr4 & 0.22796829 & $3.046679 \mathrm{e}-03$ \\
\hline Nfr_fr2 & Nsy_fr2 & 0.65734783 & $4.993796 \mathrm{e}-22$ \\
\hline & Syn_fr2 & 0.65438485 & $8.804785 \mathrm{e}-22$ \\
\hline & NcRNA_fr2 & 0.37613606 & $5.458922 \mathrm{e}-07$ \\
\hline & Nfr_fr3 & 0.27564389 & $3.116095 \mathrm{e}-04$ \\
\hline & Nsy_fr2 & 0.32334838 & $2.023643 \mathrm{e}-05$ \\
\hline Nfr_fr3 & Syn_fr2 & 0.29198808 & $1.287941 \mathrm{e}-04$ \\
\hline & Syn_fr2 & 0.97484634 & $1.716169 \mathrm{e}-109$ \\
\hline Nsy_fr2 & NcRNA_fr2 & 0.69934092 & $7.663895 \mathrm{e}-26$ \\
\hline & Syn_fr4 & 0.453322335 & $7.661126 \mathrm{e}-10$ \\
\hline Nsy_fr4 & Lof_fr4 & 0.303181013 & $6.814103 \mathrm{e}-05$ \\
\hline & NcRNA_fr2 & 0.71913446 & $7.039138 \mathrm{e}-28$ \\
\hline Syn_fr2 & & &
\end{tabular}

Since there is no gene filtering in this result, it is possible to regard these correlations as the result of intrinsic factors of exome sequencing with no correlation to the discrimination of the groups of interest. The two subsequent charts on the report show the difference in correlation for control and ASD groups. The correlations are generally similar in both groups, but stronger in the ASD group.

These findings will not be further considered for future analysis because they may be related to some specific WES characteristics and not to the discrimination parameters of the samples.

\subsubsection{Variant distribution between affected and control}




\section{groups}

The comparison for each variable between affected and control groups consists of the comparison of the quantity of variants for each individual in each group of interest (ASD and control). The variants that showed difference between ASD and control individuals at a level of significance lower than 0.05 were fra_fr2, nfr_fr4, nsy_fr2, syn_fr2 and lof_fr4. Variants with a higher mean in the affected group were nfr_fr4 and lof_fr4, and all the others had higher mean values in the control group. The pvalues for the Wilcoxon test are included in each chart on the report (Figure 23).

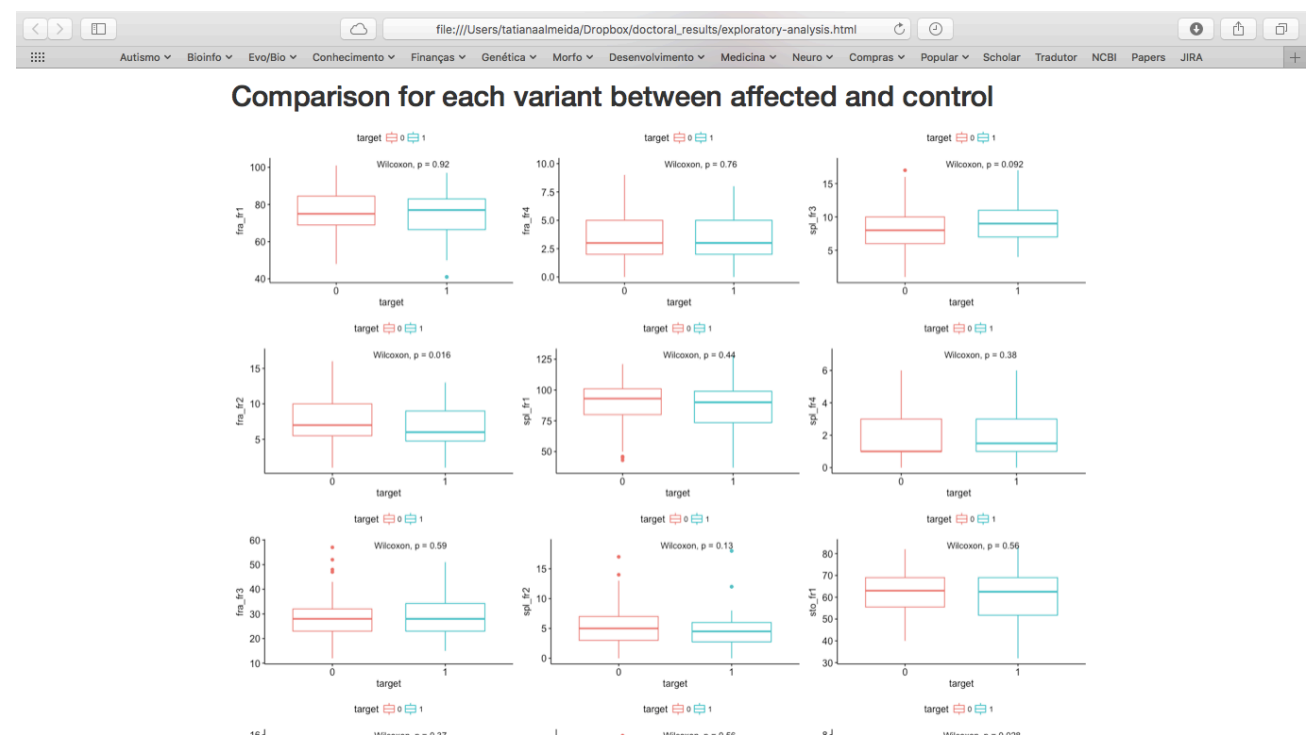

Figure 24: Screenshot of the first six charts showing the comparison for each variant between affected and control groups with the respective Wilcoxon test inside each plot.

The sum of the variables with lower mean in the ASD group (fra_fr2, nsy_fr2 and syn_fr2) maintained the discrimination between ASD and control groups (Wilcoxon $\mathrm{p}=0.0047$ ). Despite this significant $\mathrm{p}$-value, the two groups present very similar distribution (Figure 25). 


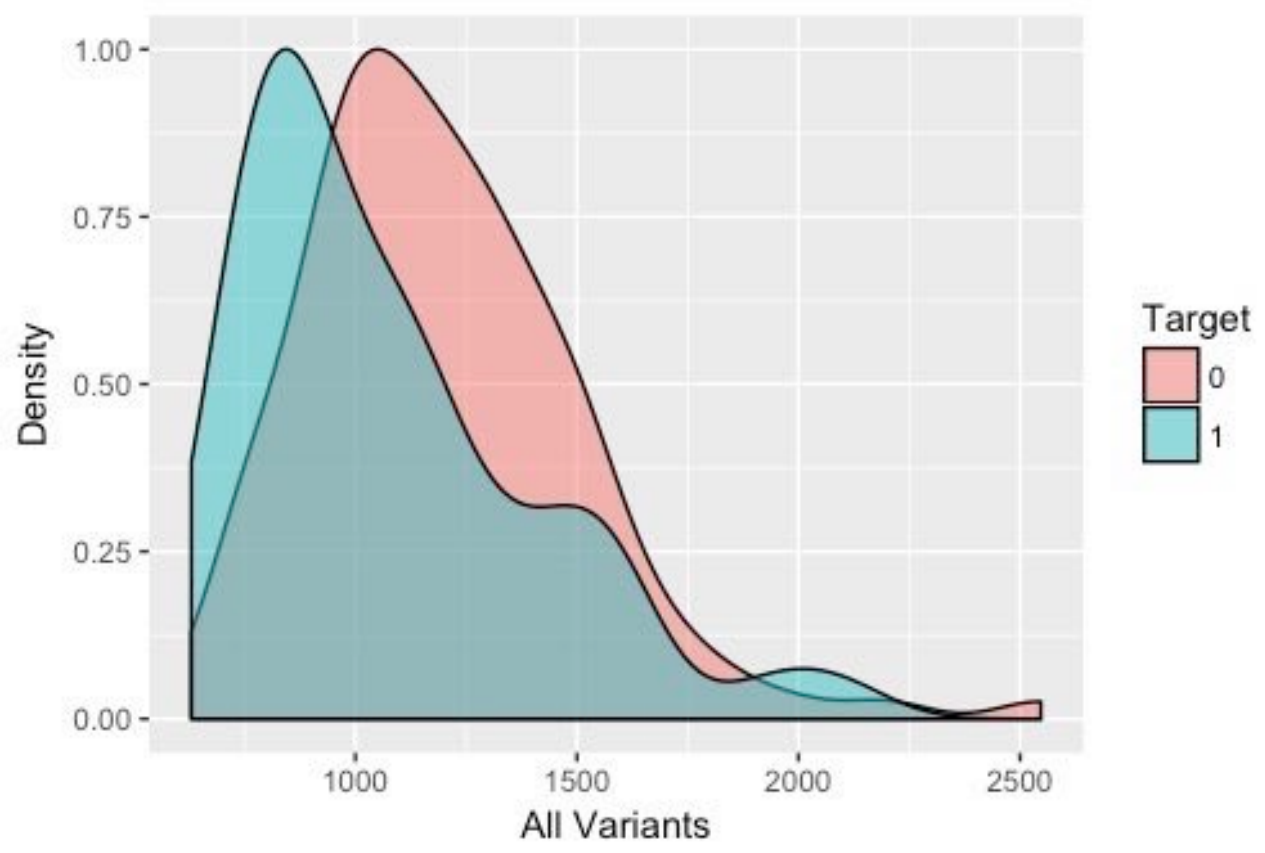

Figure 25: ASD and control (target 1 and 0, respectively) distribution of the sum of the variants fra_fr2, nsy_fr2, syn_fr2.

The sum of the variants nfr_fr4 and lof_fr4 maintains the difference between the groups (Wilcoxon $\mathrm{p}=0.002$ ). The ASD group presents a normal distribution (Shapiro-Wilk $\mathrm{p}=0.47$ ), while the control group does not (Shapiro-Wilk $\mathrm{p}=0.009$ ), and, despite this difference, the control group is entirely included in the ASD distribution, thus suggesting that this is not a good discriminant between the groups.

The importance of evaluating not only the p-value for the discriminant test, but the pattern of the distributions as well lies mostly in the fact that significance tests have limitations for heterogeneous distributions. Thus, when analyzing both distributions, the peak or the number of variants for the most representative part of the groups can be different, but most of the individuals are overlapped and this difference is caused by few individuals. Therefore, all significant differences will be analyzed concerning their distribution, and, based on the information, it will be decided whether the model could reveal a good discriminant.

The variables were also analyzed between the two Kmeans clusters and the ones that were statistically different were: $3^{\prime}$-UTR, $5^{\prime}$-UTR, ncRNA, binding sites and regulatory sites in all frequencies, splicing, miRNA in all frequencies except singletons, nsy_fr1, nsy_fr3, fra_fr3, sto_fr3, syn_fr3 and lof_fr3, nfr_fr2, syn_fr1, sto_fr4 (Figure 12). Since K-means separated the individuals mainly by the timeline of WES, it can be argued that those differences are due to the effects discussed before. The distribution of affected and control within each cluster is not statistically different (FET $\mathrm{p}=0.829$ ). For the subsequent analysis, only the variants that showed a significant difference in the first analysis will be described if they are also different between the clusters. 


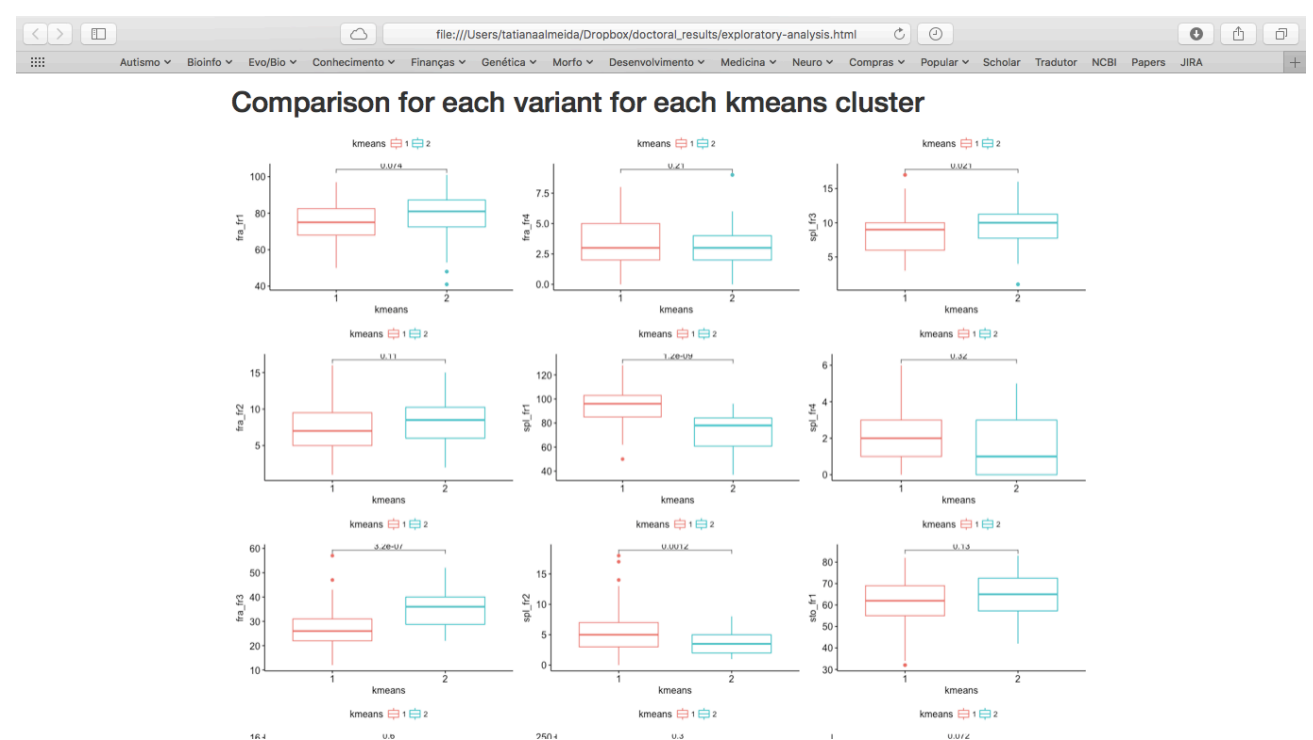

Figure 12: Screenshot of the first six charts showing the comparison for each variant between cluster 1 and cluster 2 with the respective Wilcoxon test inside each plot.

\subsubsection{Comparison Analysis}

\subsubsection{Loss-of-Function grouping}

The three approaches to grouping loss-of-function (LoF) mutations, explained in detail in Material \& Methods, did not result in any statistic difference between ASD and control groups, regarding both rare and singleton frequencies (Figure 26). The ROC curve for both frequencies did not show a better discrimination than the random choice. This corroborates the findings that there are no significant differences in total number of LoF mutation between the ASD and control groups (Figure 27). 


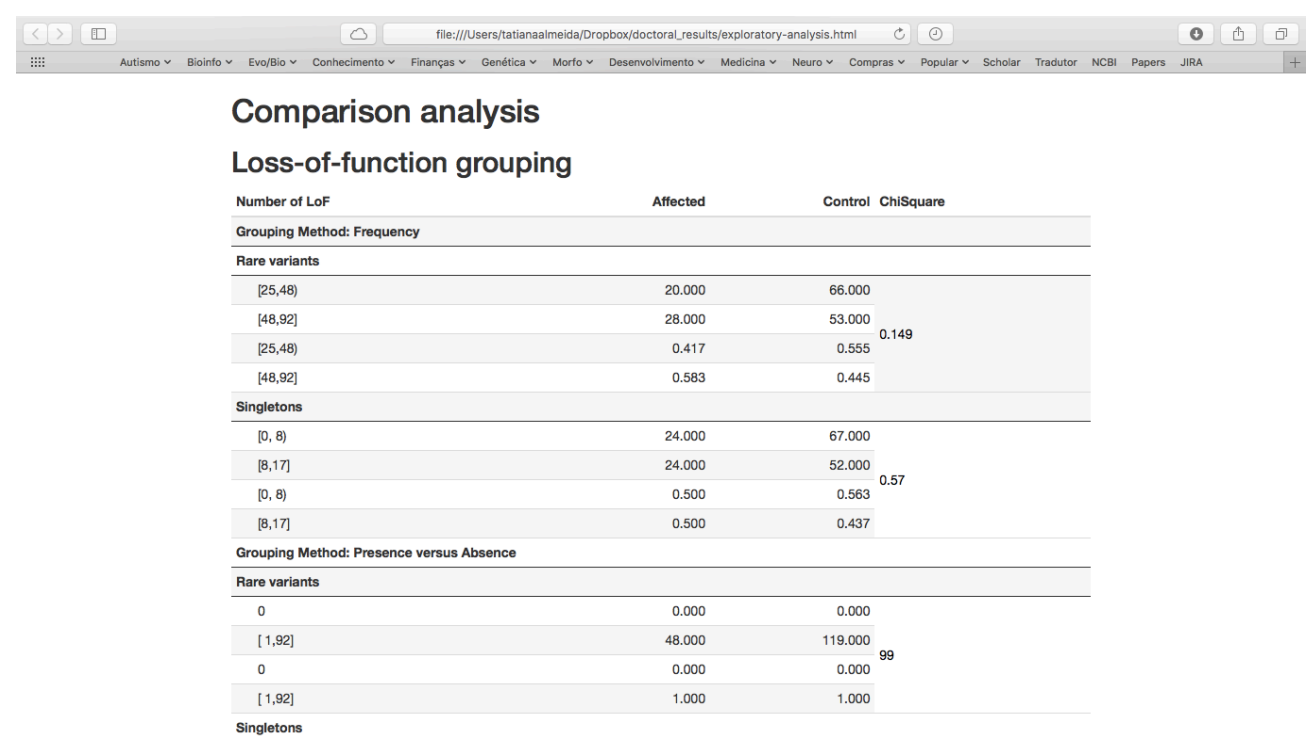

Figure 26: Screenshot of the first comparison for LoF variants on the report.

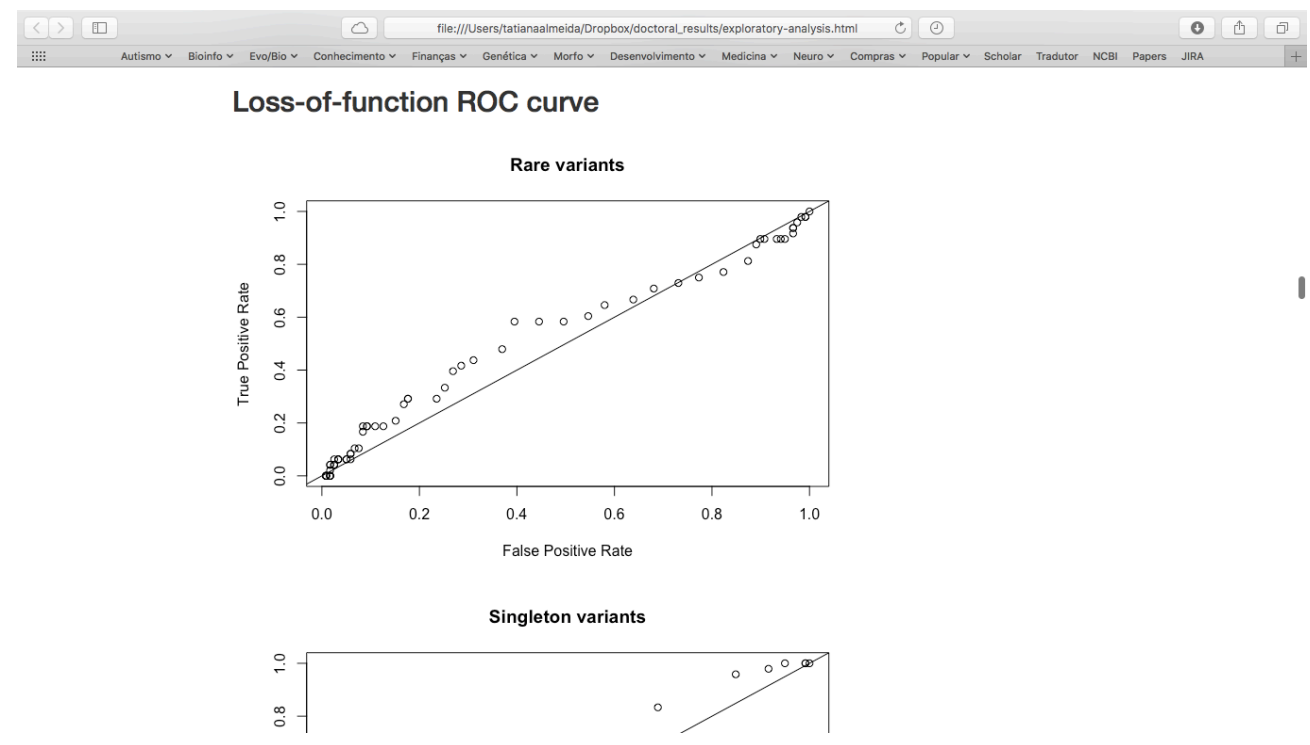

Figure 27: Screenshot of the ROC curve for all the numbers of LoF variants for this analysis.

This finding differs from the one found in the comparison for each variable between affected and control groups for the lof_fr4. The p-value for lof_fr4 is nearly nonsignificant in the first scenario, one of the reasons why this finding was not confirmed in this analysis.

\subsubsection{Multivariate models}

In this section it will be discussed the Logistic Regression, the Decision Tree, 
the Neural Network and the Support Vector Machine. No models were efficient in separating affected from controls (Figure 28).

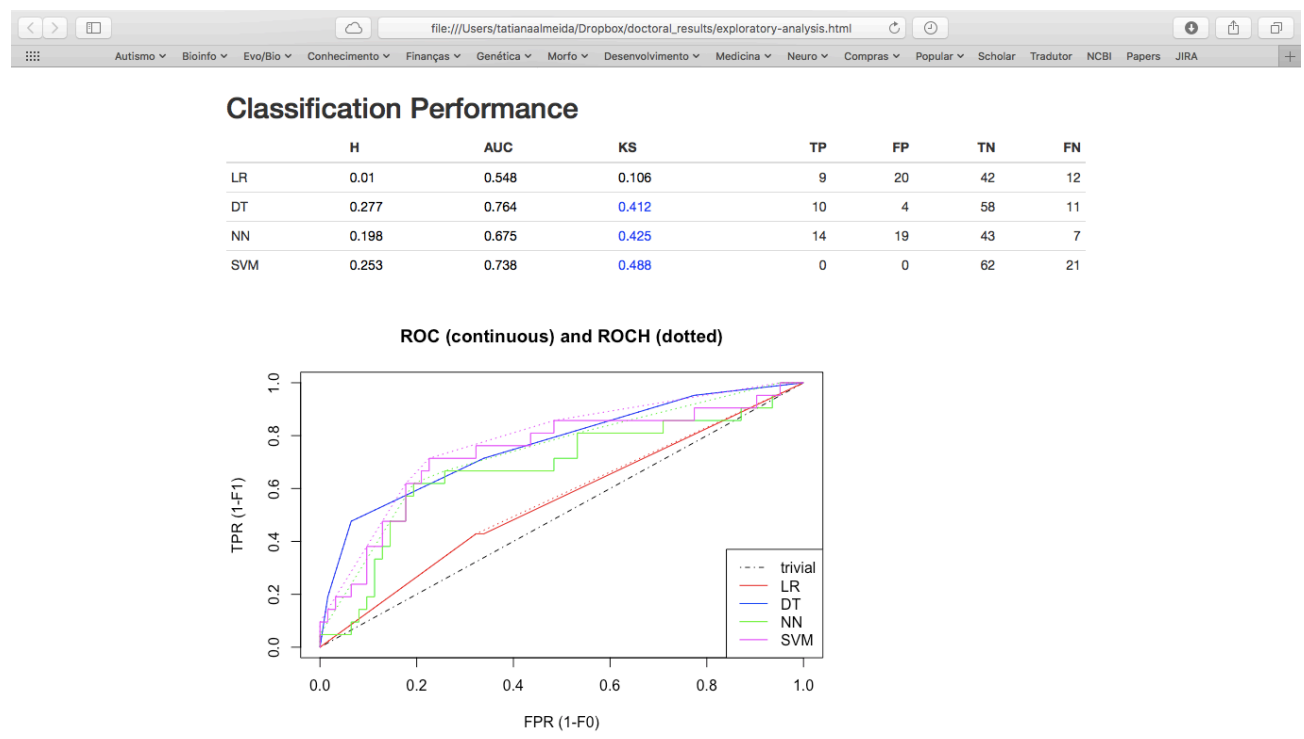

Figure 28: Screenshot of the final comparison among all four multivariate approaches to the discriminant model. This session shows the performance for the good-of-fitness metrics and the ROC curve comparison.

The best logistic regression model had 24 variants showed on the report; the decision tree model included the variants utr3_fr3, bnd_fr1 and ncRNA_fr3. The neural network and the support vector machine were the best models, but they did not reach the threshold for the $\mathrm{H}$ and AUC metrics.

\subsubsection{Principal Components Analysis}

The section - PCA with counting variants - represents the first three principal components on two charts (PCA1 vs. PCA2 and PCA2 vs. PCA3) extracted from the matrix of the number of variants for each category, example of the matrix on Attachment 22. None of charts show separation between control and ASD groups, the variables responsible for the first component are mainly regulatory variants (utr3, utr5, bnd, miRNA, ncRNA, bnd and reg), and this could be explained by the major differences in capture and alignment technique (Figure 29). This effect is removed with the coding variant analysis (described in a subsequent section), and with the visualization of the second chart with the second and third components. 


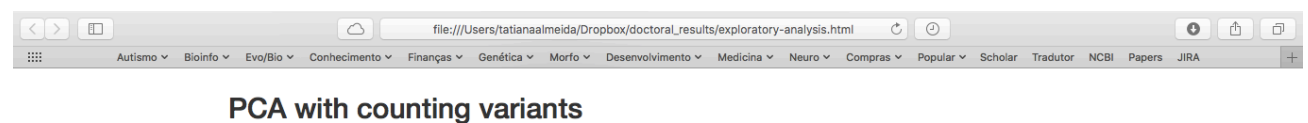

PCA with counting variants

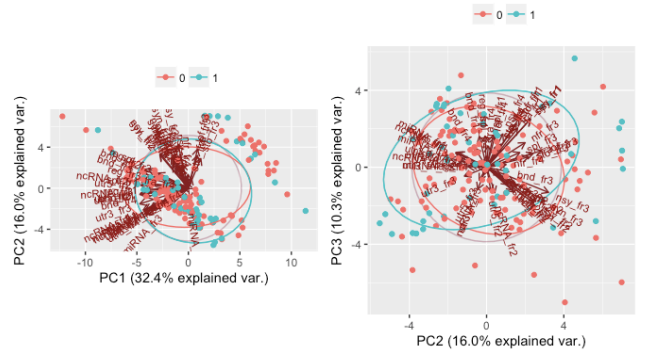

PCA with raw vef

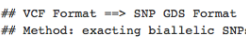

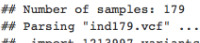

Figure 29: Screenshot of the chart of PCA with counting variants on the report.

The section - PCA with raw $v c f$ - refers to the principal component analysis showing the first three components constructed with the VCF file directly. The chart on the report shows two clear outliers: CC5098 and CC5200 (Figure 30). Figure 31 shows the PCA after both outliers were removed, and now there are three new outliers (C5054, F7935-1 and F8442-1). Figure 32 shows the first three PCs after the outliers were removed, where four other outliers remained: CC5064, CC962, F8385-1 and CC5305.

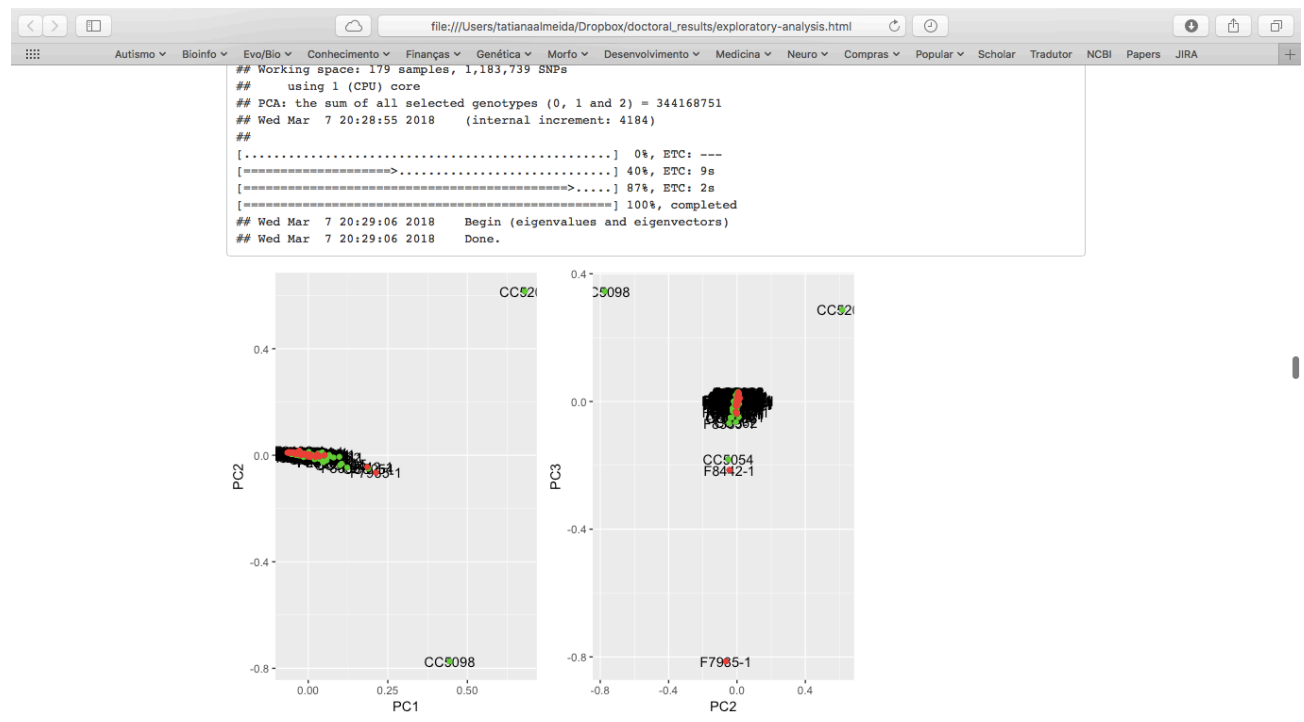

Figure 30: Screenshot of the PCA with raw VCF chart on the report. The green dots represent the control group and the red dots represent the ASD subjects. 

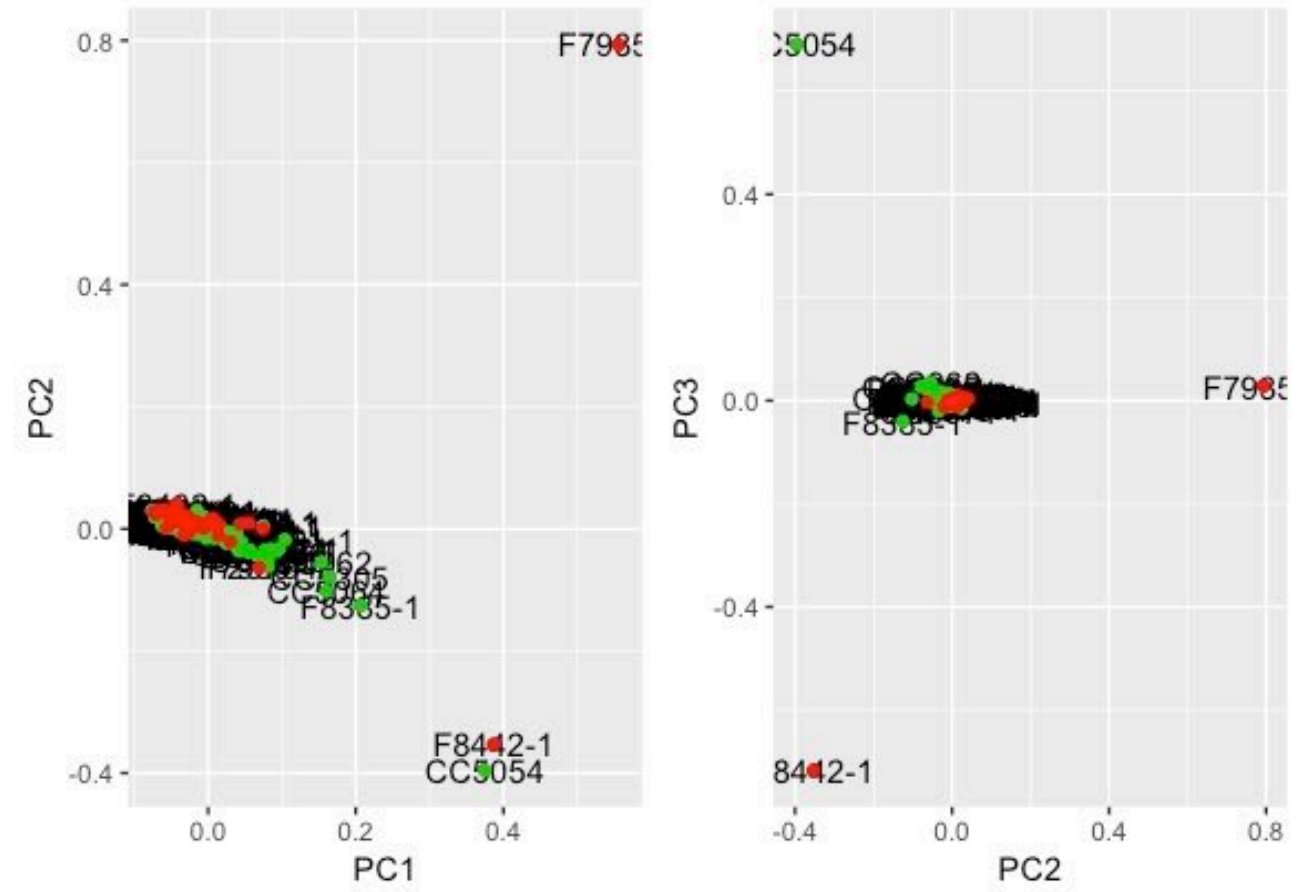

Figure 31: First three principal components from the variant call file, after removing CC5054, F7935-1 and F-8442-1. The green dots represent the control group and the red dots represent the ASD subjects.
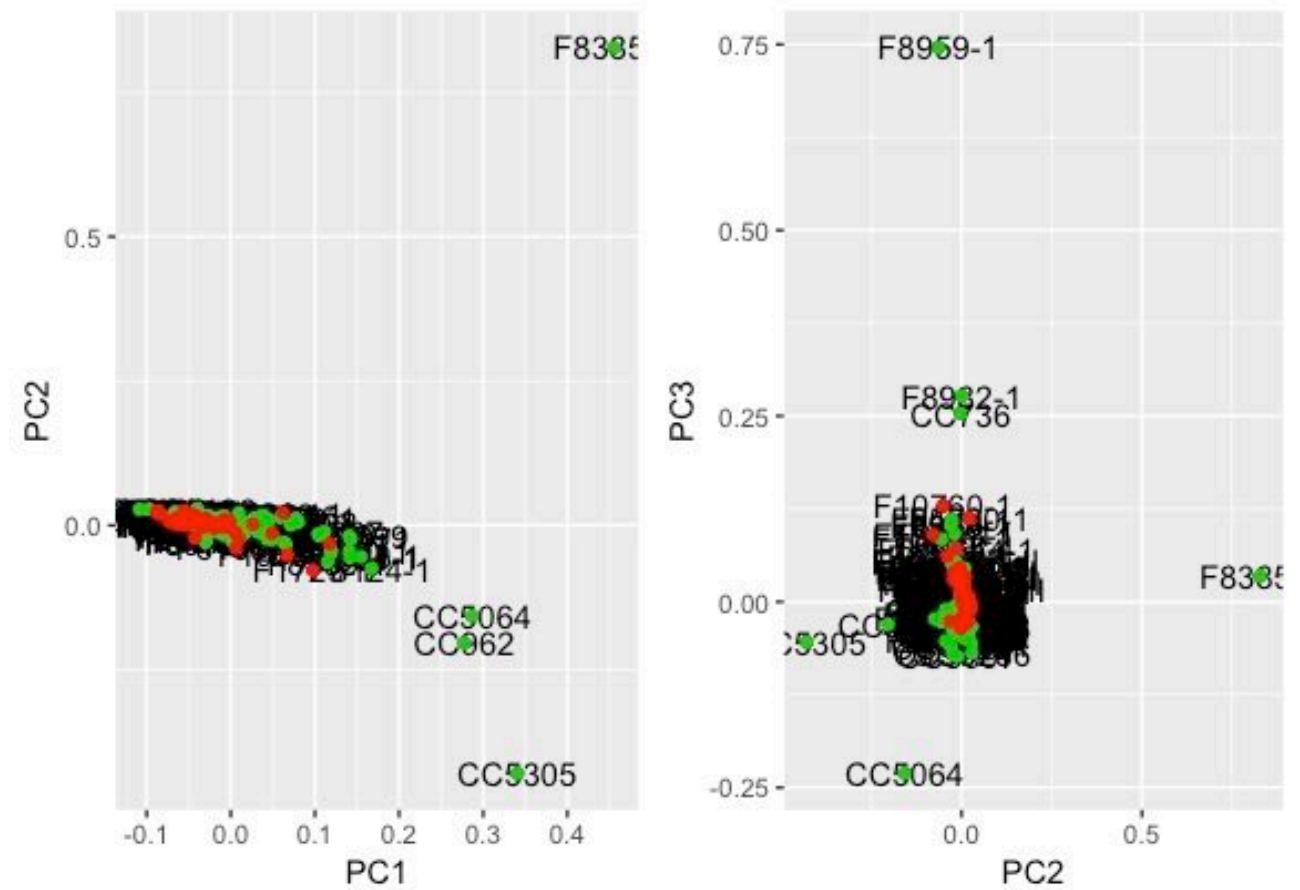

Figure 32: First three principal components from the raw vcf, after removing CC5054, F79351 and F8442-1. The green dots represent the control group and the red dots represent the ASD subjects.

After removing CC5064, CC962, CC5305 and F8385-1, a higher dispersion is noticed among the samples (Figure 33). This is the reason why outliers F8959-1, F89821 and CC736, were removed, as it can be seen on Figure 34. 

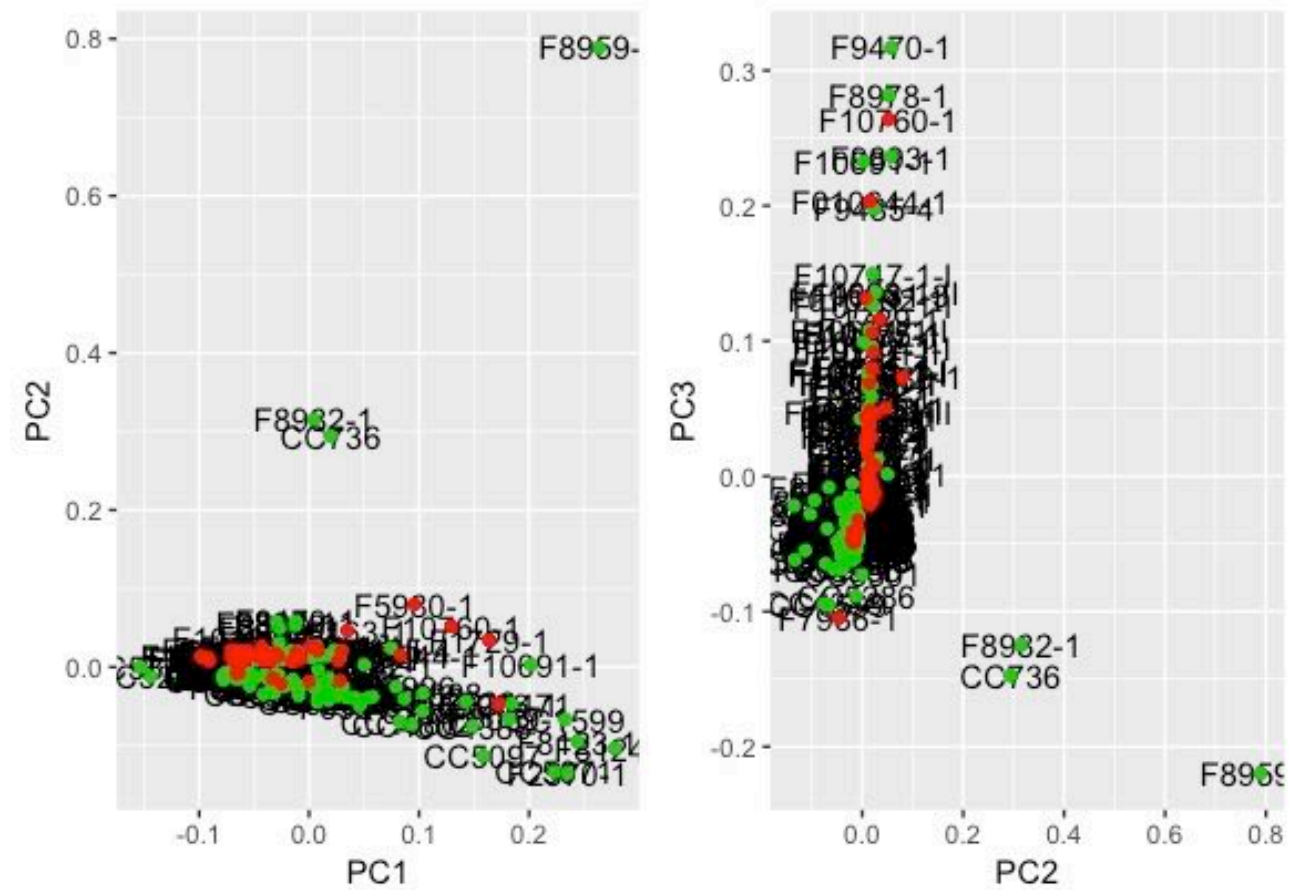

Figure 33: First three principal components from the raw VCF, after removing CC5064, CC962, CC5305 and F8385-1. The green dots represent the control group and the red dots represent the ASD subjects.
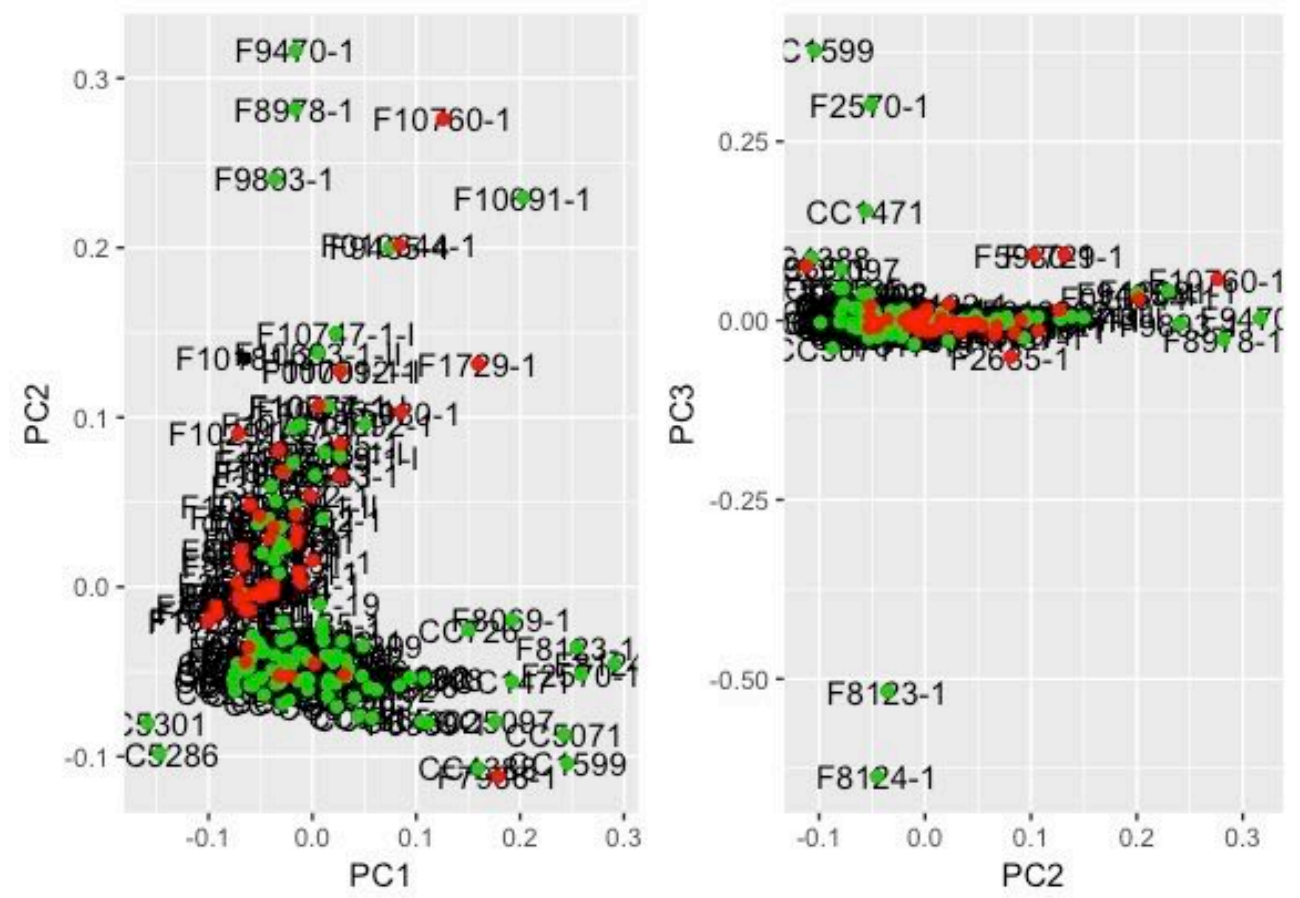

Figure 34: First three principal components from the raw VCF, after removing F8959-1, F8982-1 and CC736. The green dots represent the control group and the red dots represent the ASD subjects.

Figure 34 rreveals the tendency of different dispersion for both groups. As a 
result of the presence of many control individuals inside the ASD dispersion, the hypothesis that this difference is explained by the different WES batches was raised. Figure 35 shows the groups separated by batch number, where green is lower than 15 and red is higher than 15.

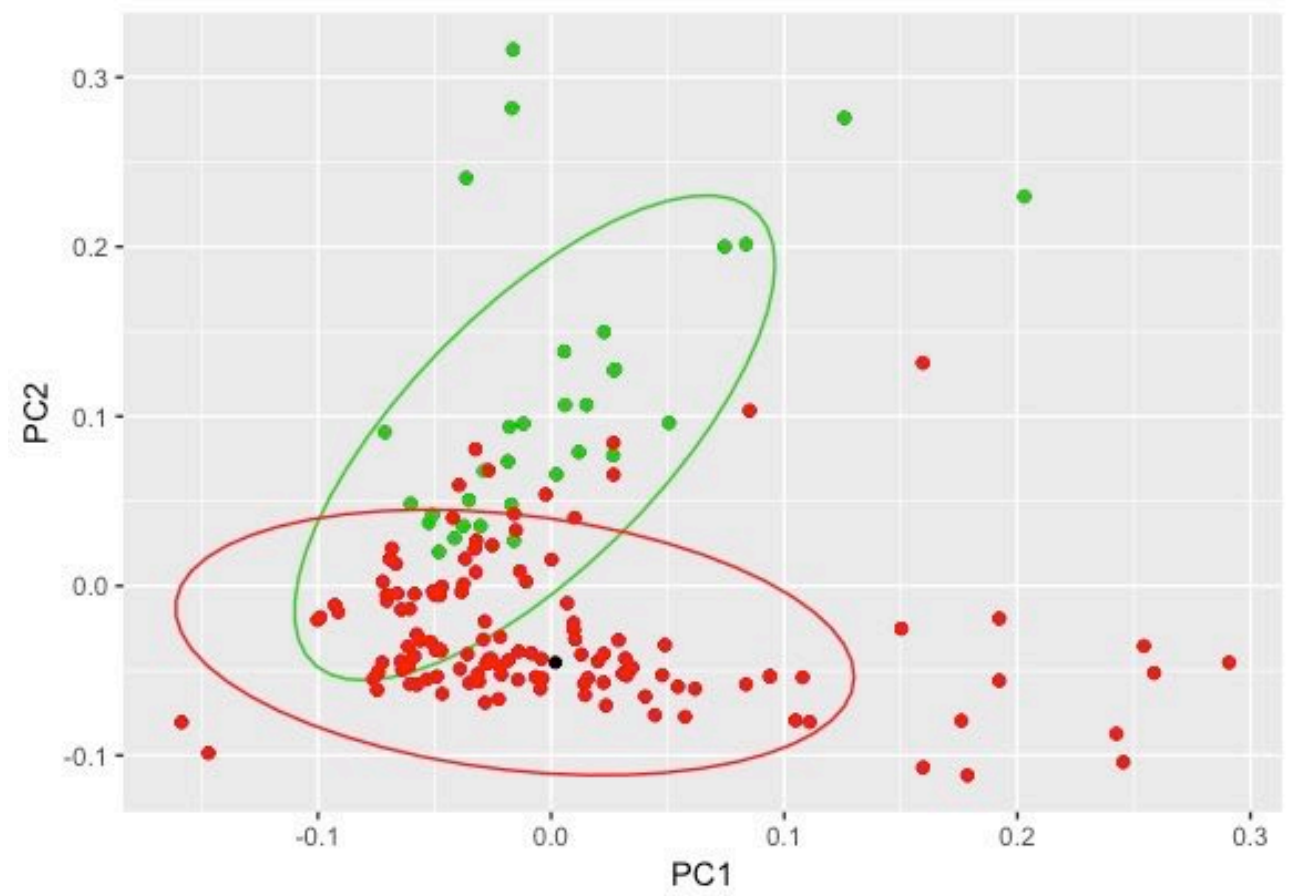

Figure 35: First two principal components from the raw VCF, the samples in green were sequenced before exome batch 15 and samples in red were sequenced after batch 15 .

Thus, if the gene list report presents a pattern that is similar to that found in this analysis, all the outliers described here will be removed, and if, after the removal, the distribution pattern remains similar to this one or more outliers are found, the investigation will stop and will only be resumed if, after removing the individuals, there is a clear different pattern between ASD and control groups.

\subsubsection{Analysis by clusters}

The kmeans separated the dataset into two clusters as previously described in Material \& Methods, where each cluster was analyzed separately by applying all the approaches above. Figure 36 sshows the beginning of the analysis of cluster 1, subsequently on the report, is that of cluster 2 . 


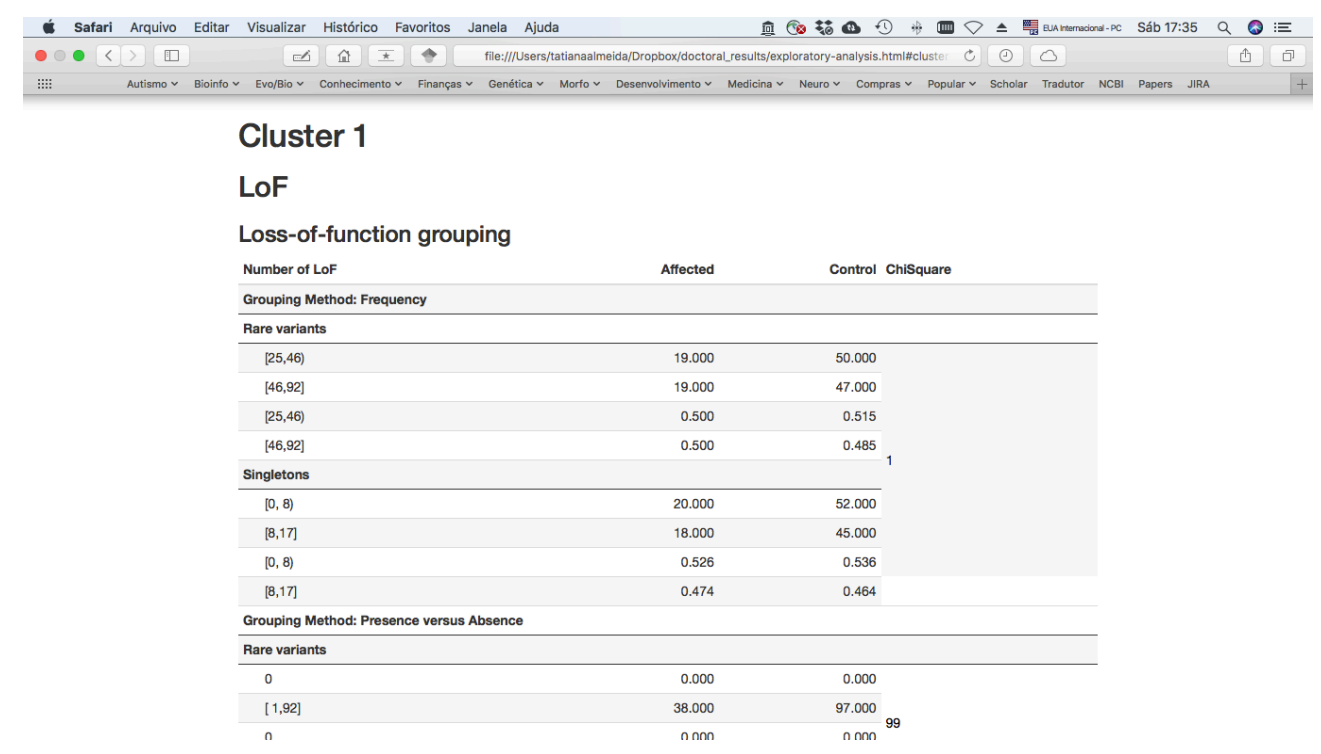

Figure 36: Screenshot of the first analysis of cluster 1 in the exploratory analysis' report.

\subsubsection{Cluster 1}

None of the analyses performed resulted in a good model.

\subsubsection{Cluster 2}

For cluster 2, both neural network and support vector machine had good models, with the AUC, KS and H metrics above the threshold. All the individuals from the testing samples were classified according to the model generated by the learning samples and received a probability value of being classified as 1 (in this case as part of the affected group). Figure 37 shows the neural network prediction value for all the tested individuals, and almost all of them received a prediction under 0.25 . The best threshold to differentiate affected from control individuals for the neural network model is 0.1 , and, within this value, there were three true positives, one false positive, ten true negatives and two false negatives for the testing set. This cluster included 22 control and 10 ASD individuals, and the low number of individuals could have interfered in the overall results. The distribution of the ASD and control individuals was very similar for this sample, thus, this cannot be regarded as a good model. 


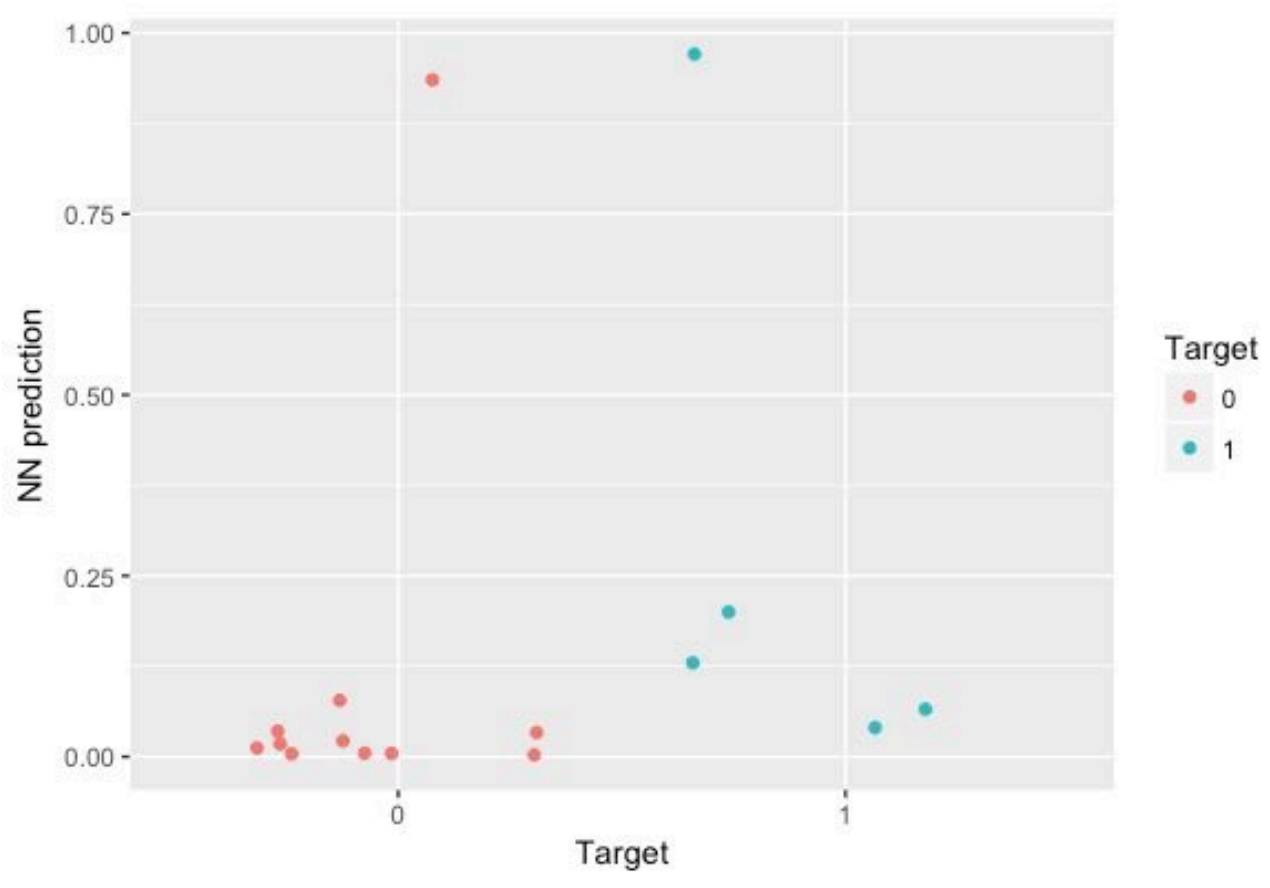

Figure 37: The neural network predicted probability for the groups, blue represents the ASD group and red represents the control group.

The best threshold for supporting the vector machine model is 0.25 , and, within this value, there are five true positives, three false positives, eight true negatives and no false negatives, for the testing set. This model had a level of sensitivity at 100\% and a level of specificity at $64 \%$. Due to the limited number of samples, it is possible that this effect is a bias for this particular sample, and further investigations are needed.

\subsubsection{Separate regions}

These sections are divided into two groups of analysis, one with only exonic regions (frameshift, canonical splicing, stopgain/loss, nonframeshift, nonsynonymous and synonymous), and another with the regulatory regions (3'-UTR, 5'-UTR, miRNA, ncRNA, regulatory sites and transcription binding sites) (Figure 38). 


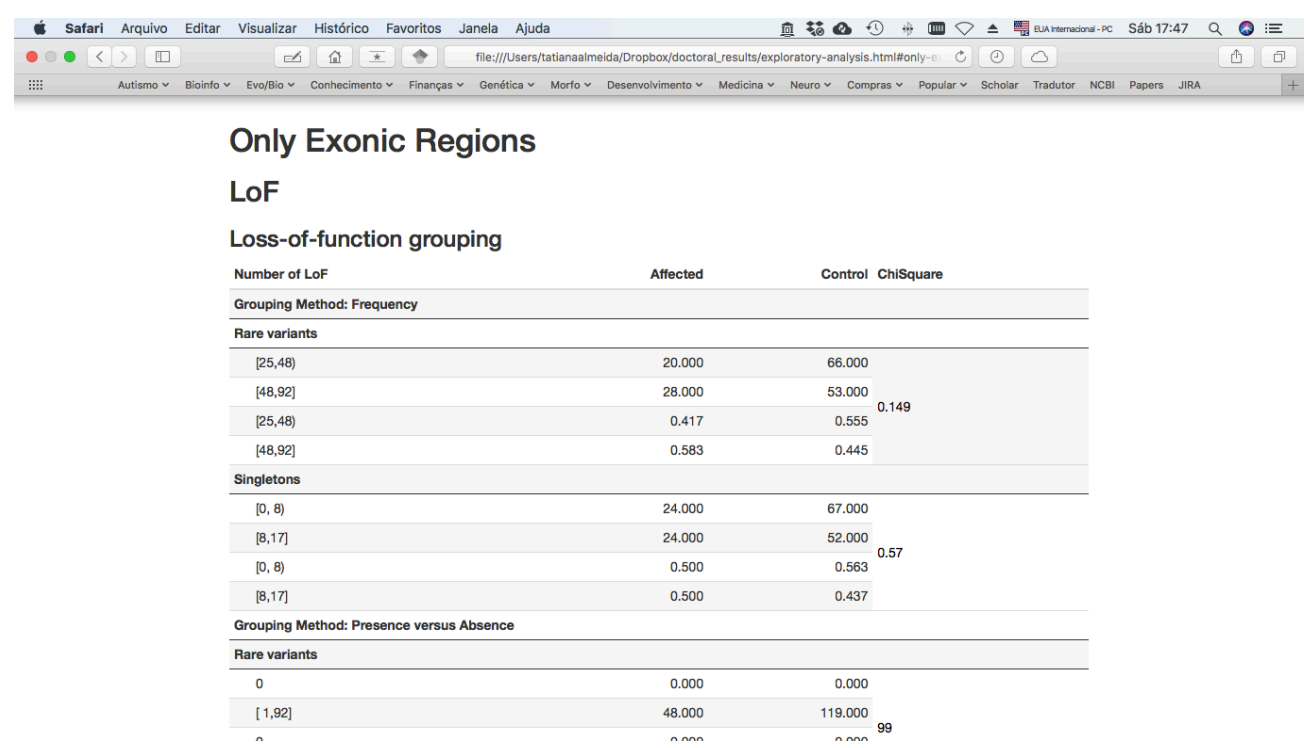

Figure 38: Screenshot of the first analysis for the Only Exonic Regions on the exploratory analysis' report.

Neither exonic nor regulatory regions individually presented a result that could distinguish the ASD group from the control group. The Decision Tree of the regulatory variants had an improvement on the final error, but only the KS metric reached the threshold, and, so, this tree will not be regarded as a good discriminant model and will not be further discussed.

In short, for this first analysis with all the genes in the whole exome sequencing, conclusions can be drawn that are relevant to the rest of the interpretations. The cluster division is mainly based on the number of regulatory variants, which are very distinct for the lower WES batches. This means that all the results concerning the different clusters should be taken carefully. The correlation among variants revealed no difference between the groups, and the correlations found could be interpreted as a characteristic of the samples. After further analysis of the variants with a possible contribution to a discriminative model, neither loss-of-function or multivariate approaches rendered good models.

\subsection{Report Analysis}

Sections 3.2.1 to 3.2.21 include the analyses of the generated report for each of the hypothesis described previously. The description will follow the same order as that in section 3.1 but will not be separated by topics. All the results within the criteria established (p-value below 0.05, $\mathrm{H}$ and KS above 0.4 and AUC above 0.8) in the previous session will be described further, and the results that do not meet the criteria will only be presented on the reports (see link on chapter 3). 


\subsubsection{Genes with critical exons}

The variants that showed difference between the groups with a significant $\mathrm{p}$ value below 0.05 , as it can be seen on the reports, were: nsy_fr2, nsy_fr3, syn_fr2, syn_fr3, and for all these variants the ASD group showed lower number of variants per individuals (lower mean). None of these variants were different between clusters (the comparison between the clusters designated during the exploratory analysis).

Variants from the same classes and all variants were summed up to test whether the significance remains for all cases. Results are presented on Figure 39.
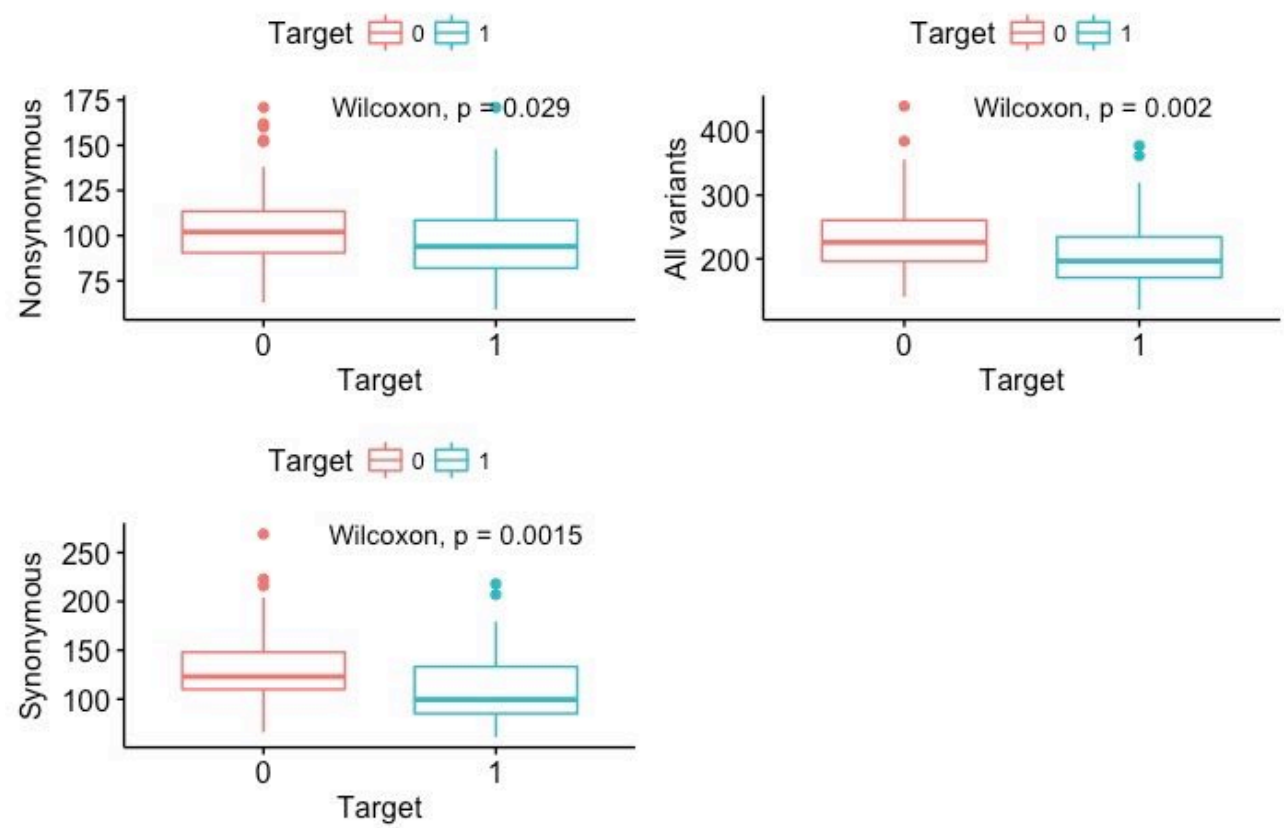

Figure 39: Boxplot of the sum of the variants and the Wilcoxon p-value. Targets 0 and 1 refer to control and to ASD groups, respectively .

Both groups presented very similar distribution (Figure 40), thus, even with a significant difference, it cannot be regarded as a good start for the discriminant model. 


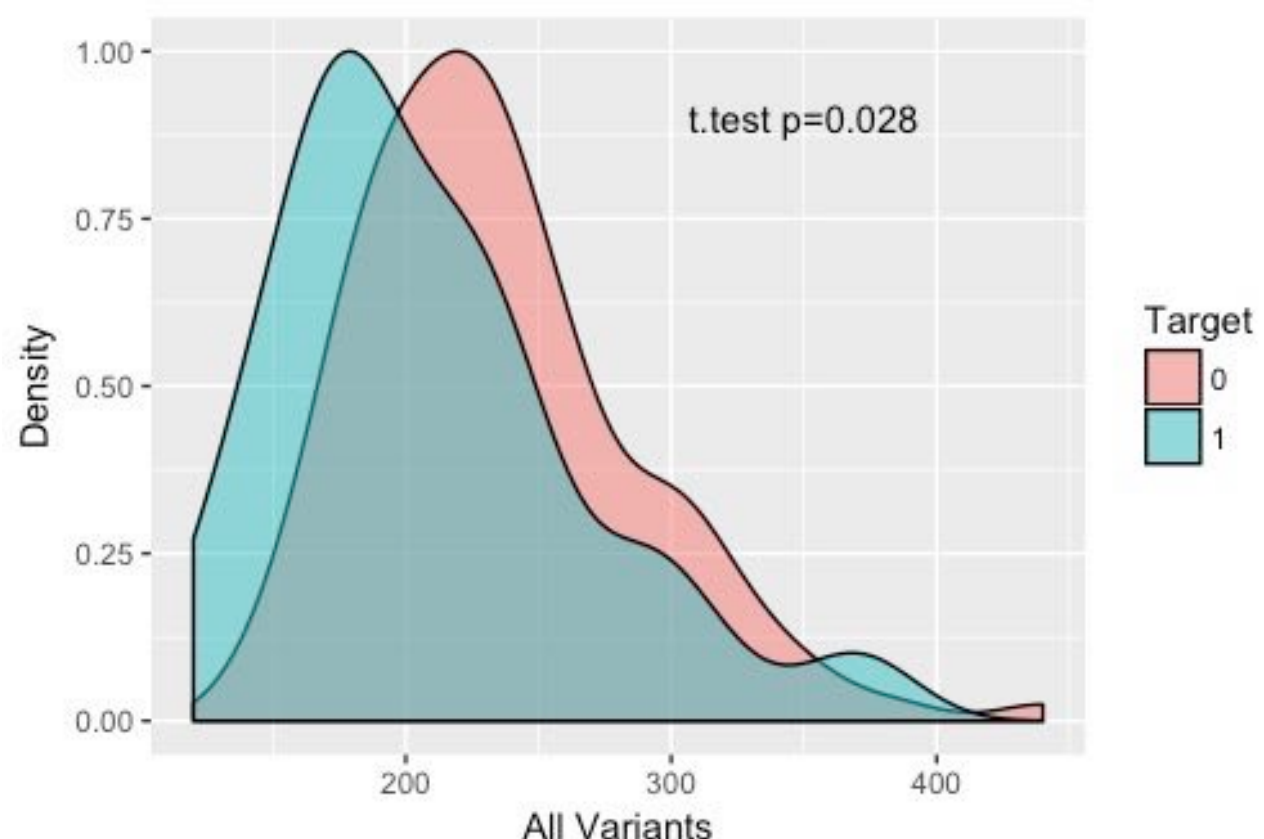

Figure 40: Distribution of all variants with a significant difference by the Wilcoxon test (nsy_fr2, nsy_fr3, syn_fr2, syn_fr3), and a t-test. Targets 0 and 1 refer to control and to ASD groups, respectively .

The LoF and missense distributions were very similar for both groups. The multivariate approaches also did not return a good discrimination model.

The principal component analysis for the variants and raw VCF showed a similar pattern found in the exploratory analysis and were not effective in separating the groups.

\subsubsection{Chromatin modifier genes}

The variants that revealed a significant difference between the affected and the control groups were sto_fr1 and spl_fr4, showing a higher mean in the ASD group. Among these variants, only sto_fr1 was different between the clusters ( $p$-values can be seen on the report for all the variants).

The sum of sto_fr1 and spl_fr4 was also different between the groups (Wilcoxon $\mathrm{p}=9 \mathrm{e}-05)$. Twenty-seven ASD individuals (57\%) had one or more variants in the sum of sto_fr1 and spl_fr4, against only 30 control individuals (25\%), the PX2 p=0.0001.

The support vector machine for cluster 2 presented all the metrics above the threshold and could discriminate the individuals for the best threshold (0.3) with level of sensitivity at $60 \%$ and level of specificity at $67 \%$. 


\subsubsection{1,527 ASD-related genes}

The variants with difference between both groups were nsy_fr2, nsy_fr3, syn_fr2. Only nsy_fr3 was different between the clusters. All variants had a lower mean in the ASD group. The sum of all the variants maintained the difference (Wilcoxon $p$-value $=0.0052$ ). The distribution of the two groups are very similar which indicates that despite the low $\mathrm{p}$-value this parameter is not a good discriminant for the groups (Figure 41).

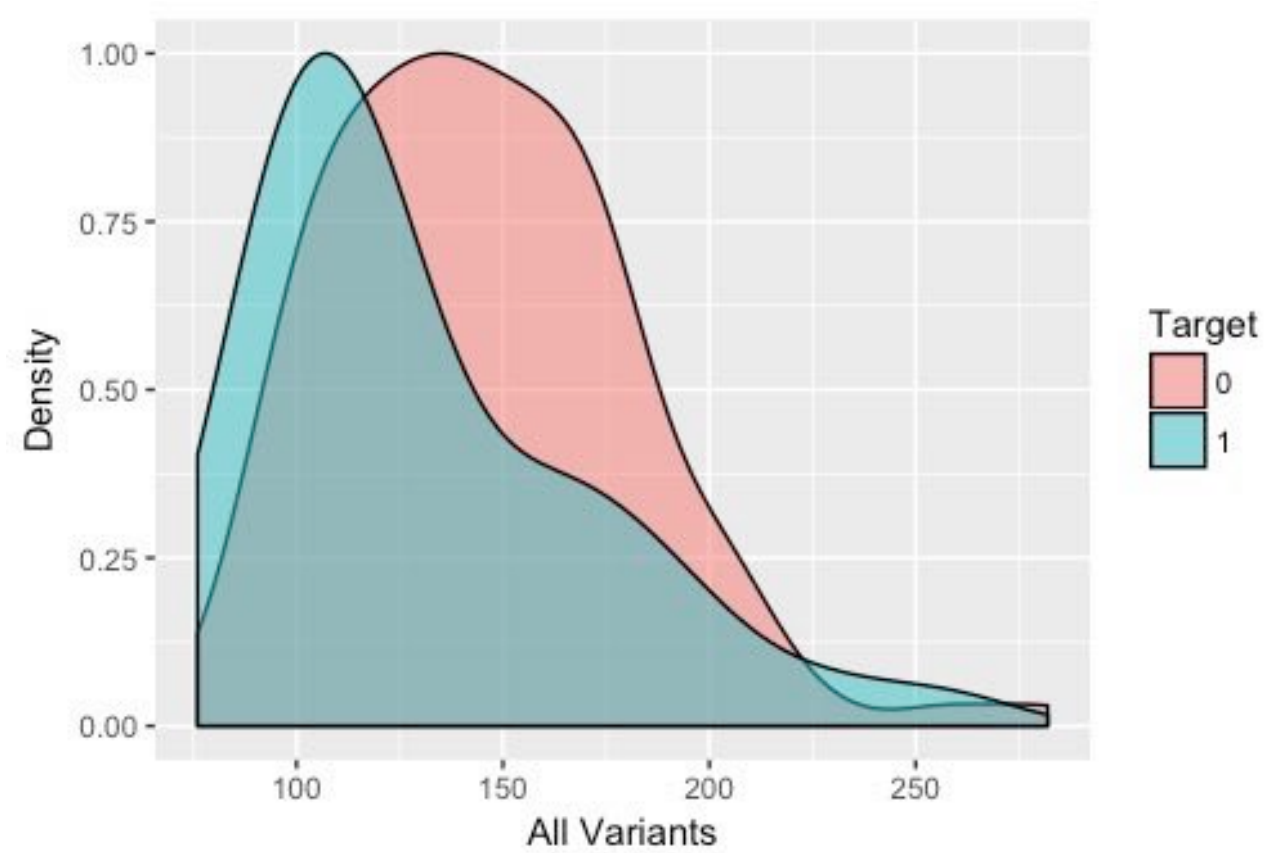

Figure 41: The distribution of the sum of nsy_fr2, nsy_fr3, syn_fr2 variants for both groups.

Targets 0 and 1 refer to the control group and to the ASD group, respectively.

The support vector machine for cluster 2 was a good discriminant approach with AUC above 0.8, KS and $\mathrm{H}$ above 0.4. With a threshold of 0.328 , with level of sensitivity at $100 \%$ and level of specificity at $90 \%$.

\subsubsection{FMRP targets}

The variants that separated the groups of interest were fra_fr1, sto_fr1, nsy_fr2, syn_fr2, syn_fr3. The fra_fr1, nsy_fr2, syn_fr2, syn_fr3 variants showed a lower mean for the ASD group, and the sto_fr1 variant showed a higher mean for the ASD group. Among these variants, the only ones that remained with this difference between the clusters were fra_fr1, sto_fr1 and syn_fr3.

The sum of fra_fr1, nsy_fr2, syn_fr2, syn_fr3 variants maintained the difference between ASD and control groups (Wilcoxon $\mathrm{p}=0.011$ ). Nevertheless, the distribution 
of both groups is very similar and cannot be regarded as being a good discriminant model (Figure 42).

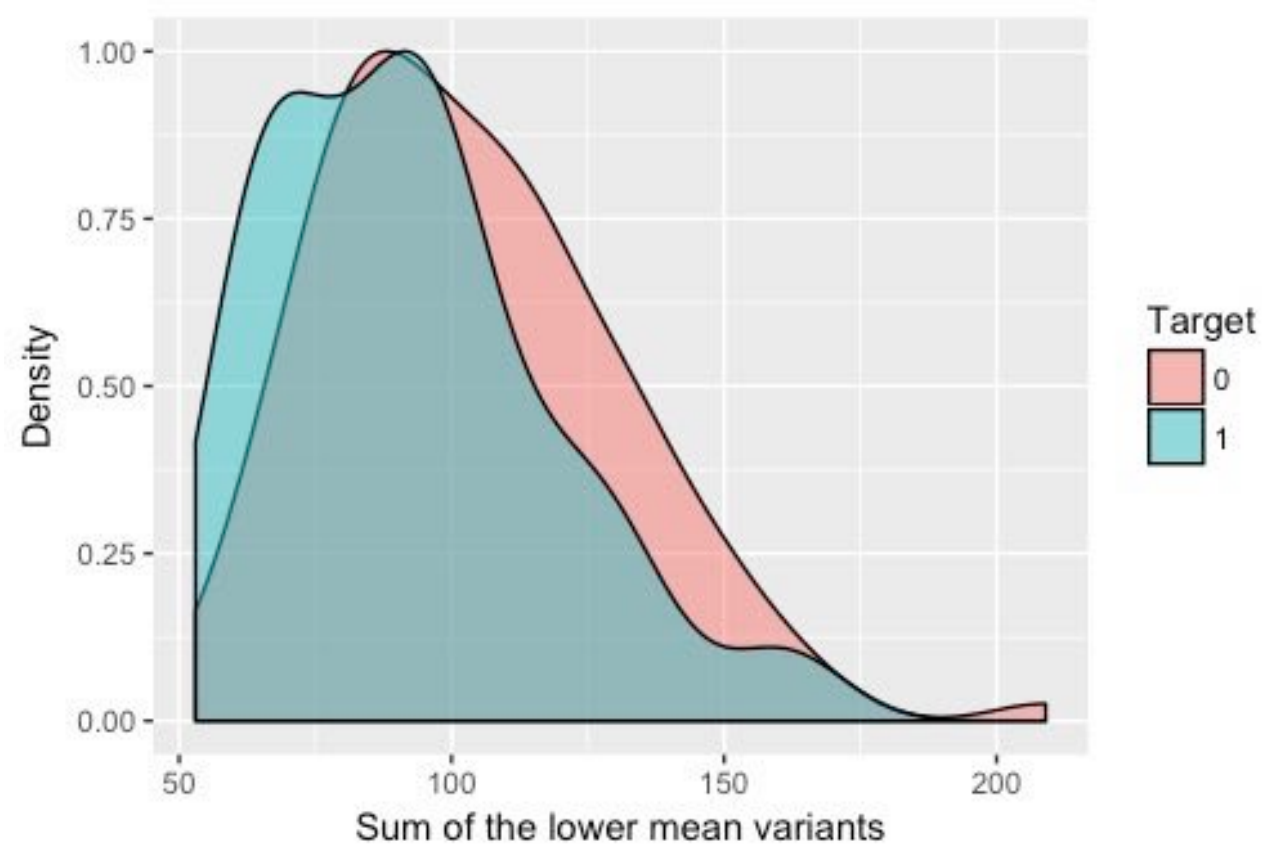

Figure 42: The distribution of the sum of lower mean variants in WES. Targets 0 and 1 refer to control and to ASD groups, respectively.

Figure 43 shows the sto_fr1 distribution for both groups, and the analysis of the number of individuals with more or less than two variants returned a non-significant $\mathrm{p}$-value (PX2 p=0.09).

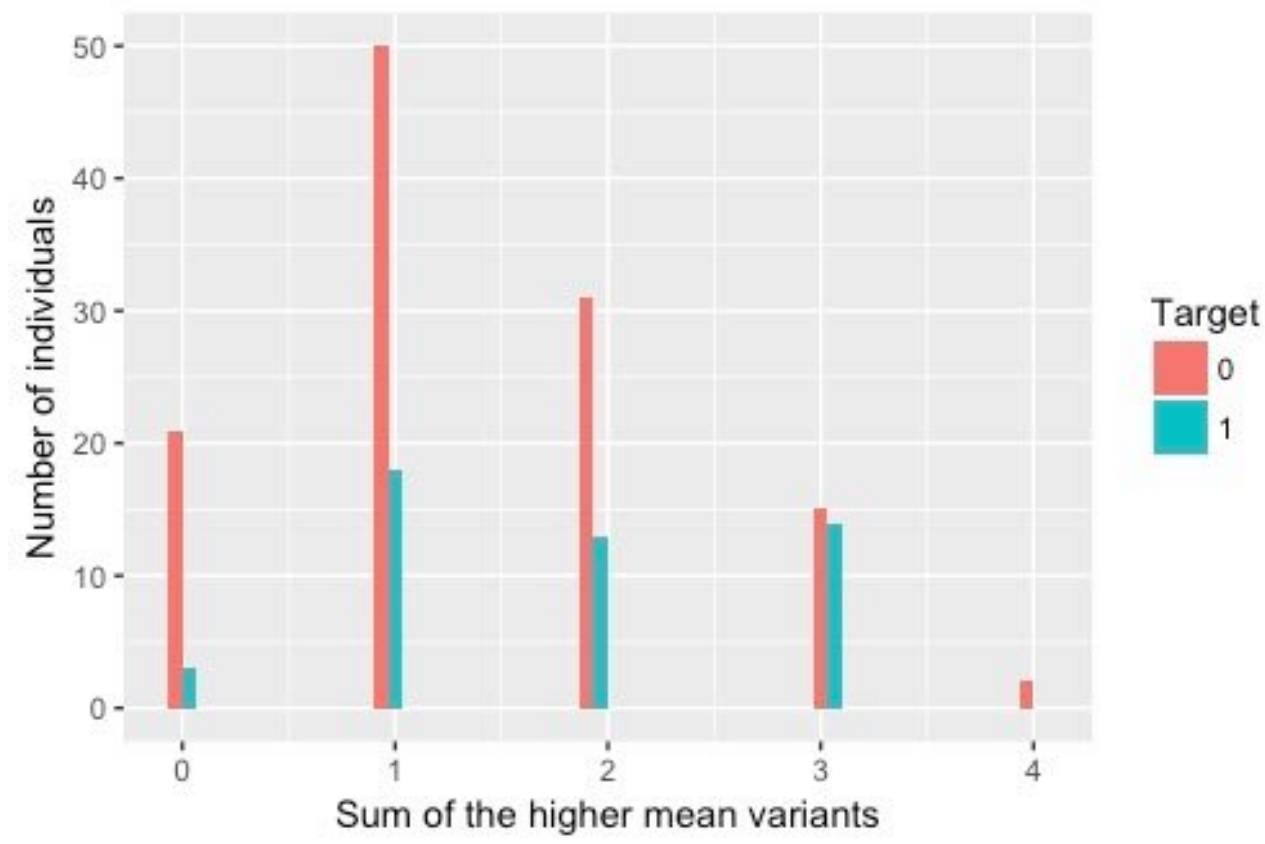

Figure 43: The histogram of the sto_fr1 in both groups. Targets 0 and 1 refer to control and to ASD groups, respectively. 
Cluster 2 showed a significant difference between the number of rare LoF mutations, with $77 \%$ of the control group presenting no mutations versus $80 \%$ of the ASD group presenting one or more mutations. This result confirms the pattern expected in the literature, but when applied to the whole number of individuals this signal is no longer significant.

\subsubsection{ASD-related genes}

The variants that revealed a significant difference between ASD and control individuals were sto_fr1, sto_fr4 and nsy_fr3. The nsy_fr3 variant showed a lower mean for the ASD group, and the sto_fr1 and sto_fr4 variants showed a higher mean for the same group. The variant that remained with significant difference between the clusters was sto_fr1.

Despite the significance in the Wilcoxon test for nsy_fr3, the distribution of the variants between both groups is very similar and further analysis will not be pursued.

The sum of sto_fr1 and sto_fr4 maintains the higher mean in the ASD samples (Wilcoxon $\mathrm{p}=0.0002$ ). In this sum of sto_fr1 and sto_fr4, the control group presents $75 \%$ of the individuals with no variant, whereas the ASD group presents $44 \%$ of them with one or more variants per individual, PX2 p=0.0002 (Figure 44).

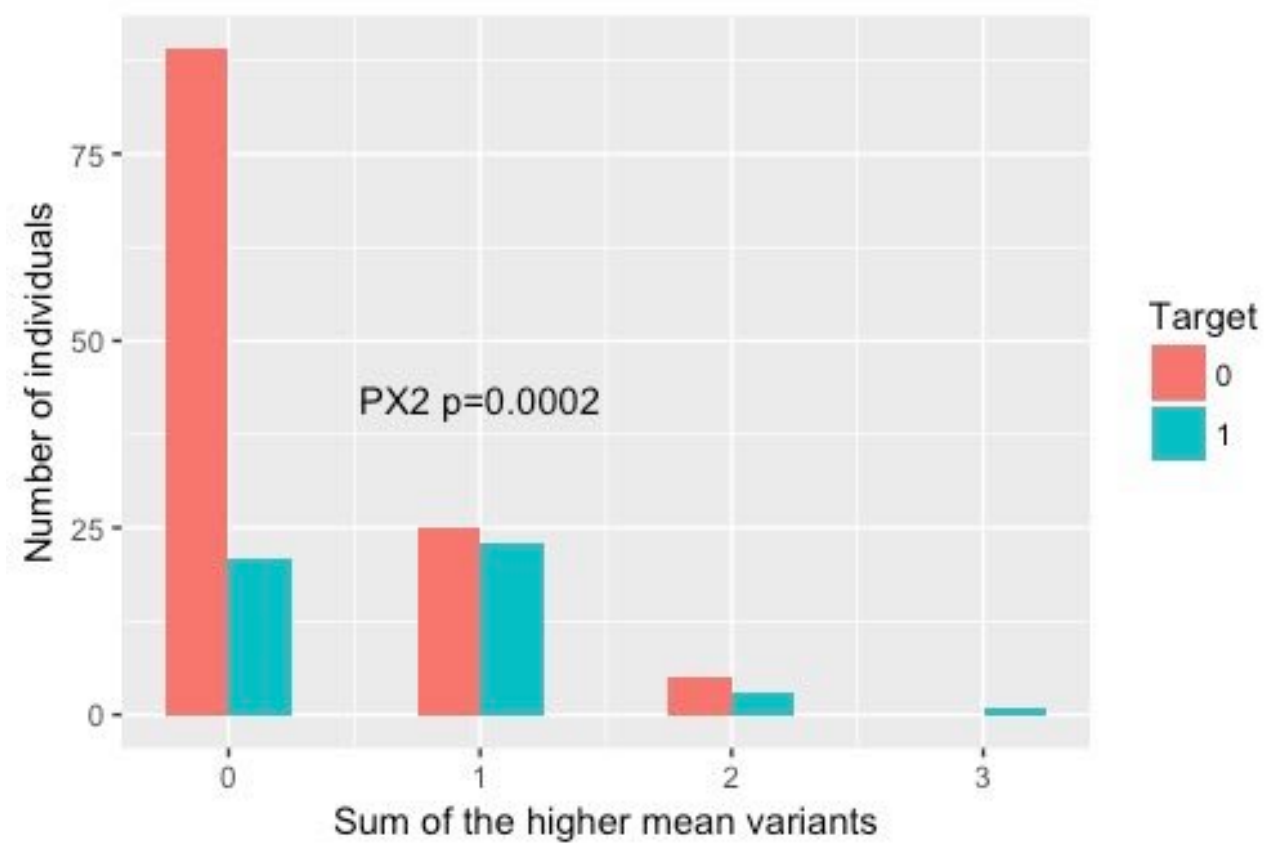

Figure 44: The histogram of the sum of sto_fr1 and sto_fr4.

\subsubsection{GABA pathway}


No analysis presented a significant result for this gene list.

\subsubsection{Hipermutable Genes}

The variants that revealed a significant difference between affected and control groups were nfr_fr3 and nfr_fr4, with a higher mean for the ASD group. None of the variants were different in the clusters.

The sum of the nfr_fr3 and nfr_fr4 maintained the difference (Wilcoxon $\mathrm{p}=0.019$ ). Figure 45 shows the distribution of these variants in both groups, with the the ASD group with $31 \%$ of its individuals presenting one or more variants against the $16 \%$ presented by the control group, but the significance was only marginal (PX2 $\mathrm{p}=0.045)$.

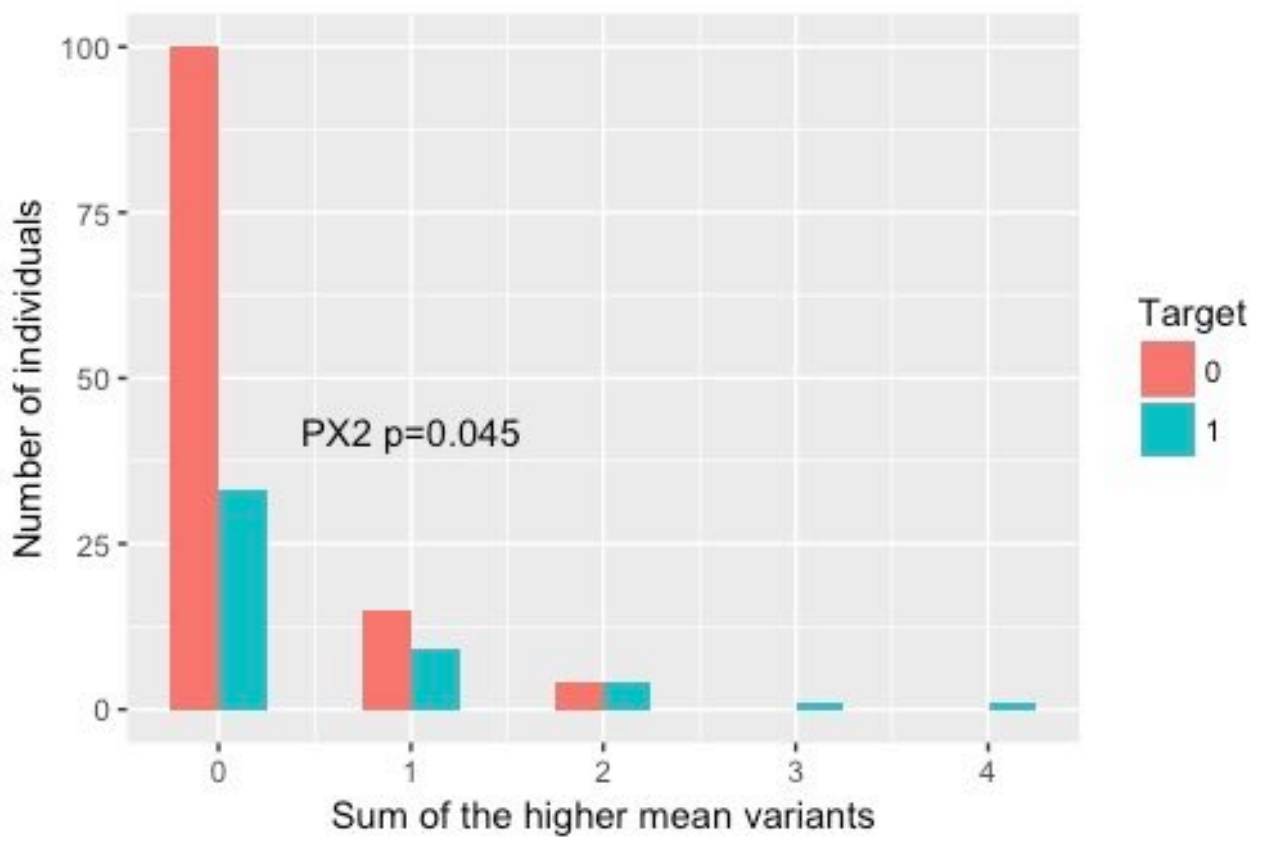

Figure 45: Histogram of the sum of nfr_fr3 and nfr_fr4 for the control and ASD groups.

\subsection{8 mTOR Pathway}

The variants that revealed a significant difference between affected and control groups were nsy_fr2, with a lower mean for the ASD group.

Regarding nsy_fr2, the control group had $46 \%$ of its individuals with one or more nsy_fr2 variants, versus 25\% presented by the ASD group (PX2 $\mathrm{p}=0.014)$. 
Despite the values of $\mathrm{H}, \mathrm{KS}$ and AUC were above the threshold for the cluster 1 logistic regression, and none of the parameters of the regression had a p-value under 0.05 , which means that this model will not result in a good discriminant model.

\subsubsection{IGF1 Pathway}

The support vector machine for cluster 2 was a good discriminant approach with AUC above 0.8, KS and $\mathrm{H}$ above 0.4. With a threshold of 0.5 , level of sensitivity at $60 \%$ and level of specificity at $90 \%$.

\subsubsection{Genes intolerant to loss-of-function mutations}

The variants that revealed a significant difference between affected and control groups were sto_fr1, nsy_fr2, syn_fr2 and syn_fr3, all of them with lower mean for the ASD group. For the cluster comparison, from the variants above, the one that showed a significant difference was syn_fr3.

The sum of all these variants maintained the difference (Wilcoxon $\mathrm{p}=0.0021$ ), the distribution pattern of both groups, as seen in previous analyses by the graphic dispersion, is very similar, which will not return a good discriminant model.

The neural network for cluster 2 was a good discriminant approach with AUC above $0.8, \mathrm{KS}$ and $\mathrm{H}$ above 0.4 . With a threshold of 0.03 , level of sensitivity was $100 \%$ and level of specificity was $80 \%$. Figure 46 shows the possible distributions for both groups, and, because of the proximity between the ASD and control groups, this model has to be considered carefully. 


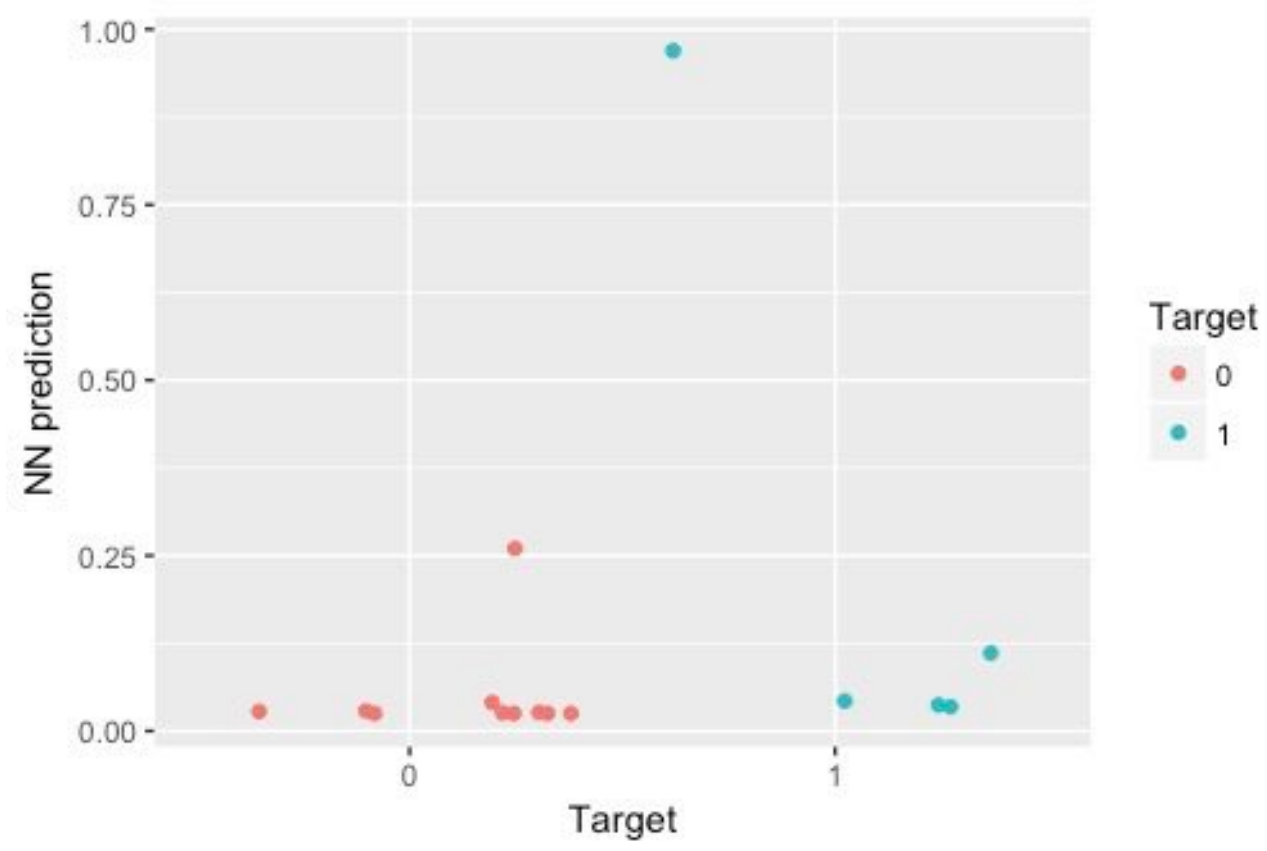

Figure 46: The neural network predicted probability for both groups, where blue represents the ASD group and red represents the control group.

\subsubsection{Differentially expressed genes M1}

The variants that revealed a significant difference between affected and control groups were sto_fr3, with a lower mean for the ASD group. Despite the significant difference, the two distributions are very similar to one another, and no discriminant model can be constructed based this data.

The support vector machine for cluster 2 had a discriminant model with AUC above $0.8, \mathrm{KS}$ and $\mathrm{H}$ above 0.4 . With a threshold of 0.5 , level of sensitivity and level of specificity at $80 \%$.

\subsubsection{Differentially expressed genes M2}

The variants that revealed a significant difference between affected and control groups were fra_fr4 and nsy_fr2. Variants nsy_fr2 with a lower mean for the ASD group and fra_fr4 a higher mean for the same group. None of them were different in the cluster comparison.

Despite the difference between ASD and control groups when it comes to nsy_fr2 variants, the distribution of those variants is very similar, which will not return a good discriminant model.

Regarding the fra_fr4 variant, only one control individual presented one 
singleton frameshift variant (CC245 (0.8\%)), in comparison to six ASD individuals with one or more variants (12\%), PX2 p=0.002 (Figure 47). If we add the other LoF variants like stopgain/loss and/or splicing, this signal decreases. With only the addition of splicing singleton variants $(\mathrm{PX} 2 \mathrm{p}=0.04)$ and the addition of stopgain/loss $(\mathrm{PX} 2 \mathrm{p}=0.05)$ it disappears. All three variants together are seen on LoF table with PX2 $\mathrm{p}=0.11$.

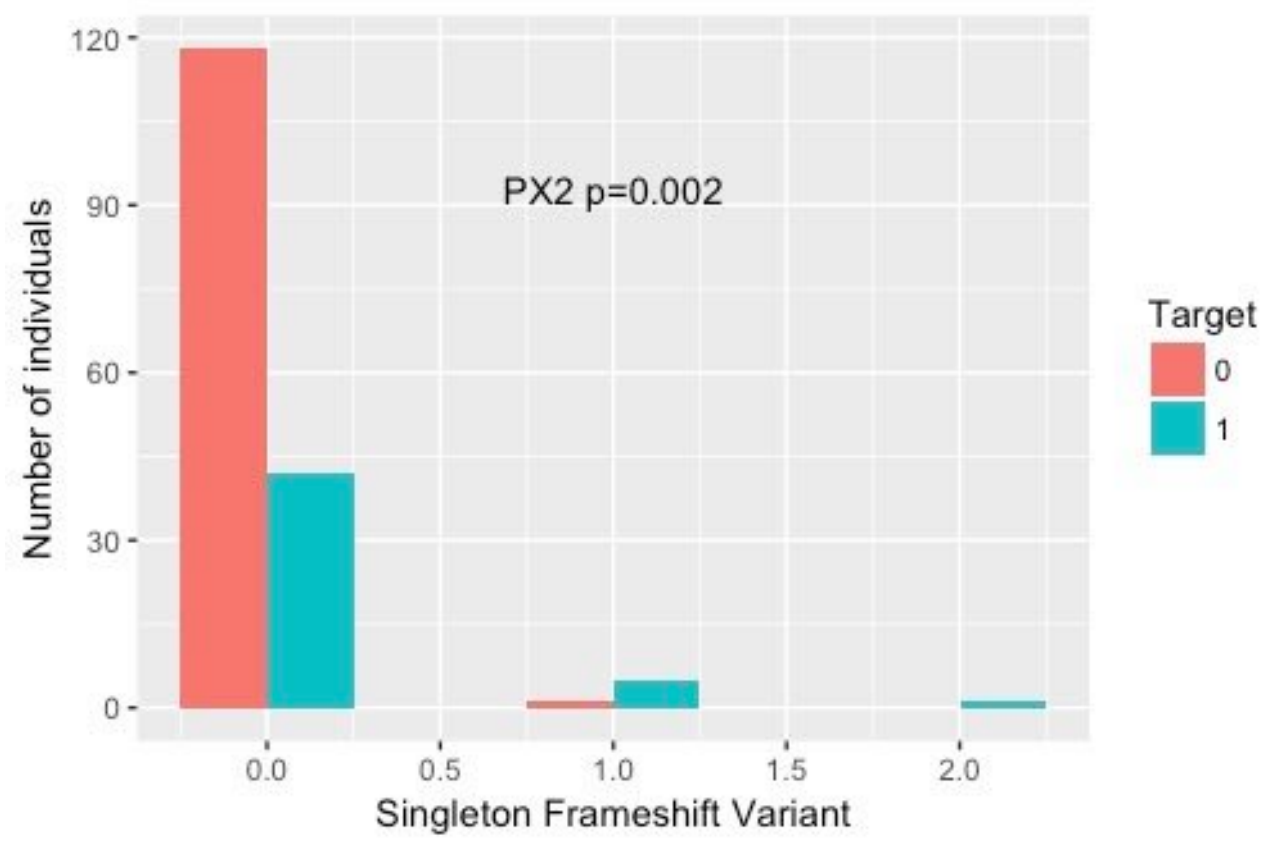

Figure 47: Histogram of Singleton frameshift distribution in the GOI.

The support vector machine for cluster 2 was a good discriminant approach with AUC close to $0.8, \mathrm{KS}$ and $\mathrm{H}$ above 0.4 . With a threshold of 0.55 , level of sensitivity at $80 \%$ and level of specificity at $92 \%$.

\subsubsection{Differentially expressed genes M8}

The variants that revealed a significant difference between the affected and the control groups were nsy_fr3, with a higher mean for the ASD group.

The distribution of the rare nonsynonymous variants showed $93 \%$ of individuals in the control group with three variants or less, while $73 \%$ of the ASD group fell within that category, with the other $27 \%$ with four to ten variants per individual (PX2 p=0.0008).

\subsubsection{Differentially expressed genes M16}


The variants that revealed a significant difference between affected and control group were nfr_fr4, with a higher mean for the ASD group.

Regarding the singleton nonframeshift variant, two individuals in the ASD group had one variant against none in the control group, and this difference draws attention because of the uniqueness of the ASD group, but it is not sufficient to generate a significant difference (PX2 $\mathrm{p}=0.08)$ and could be the result of biased sequencing.

The support vector machine for cluster 2 was a good discriminant approach with AUC close to $0.8, \mathrm{KS}$ and $\mathrm{H}$ above 0.4 . With a threshold of 0.55 , level of sensitivity at $100 \%$ and level of specificity at $58 \%$.

The principal component analysis for cluster 2 revealed a slight difference in PC1 and PC2 between the groups. After removing five outliers, the pattern seems to be consistent with two different groups, mainly for PC1 (Figure 48).

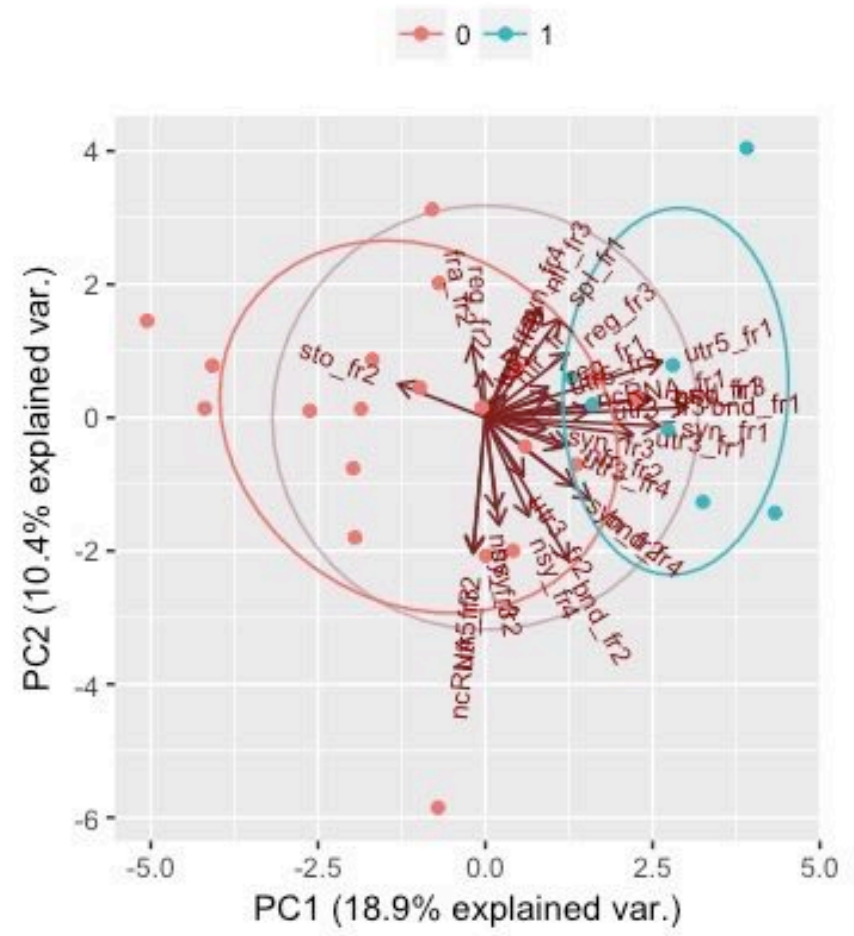

Figure 48: First two PC showing the dispersion for both GOI. Blue represents the ASD group and red represents the control group.

The variants that are mainly responsible for this discrimination are as follows: bnd_fr1, utr5_fr1, syn_fr1, nsy_fr1, bnd_fr3, utr3_fr1 and sto_fr2. These variants were used to build a principal component analysis for cluster 1, and, as seen in Figure 48, these same variants are not responsible for the differentiation in PC1, and cannot be applied to this group. 


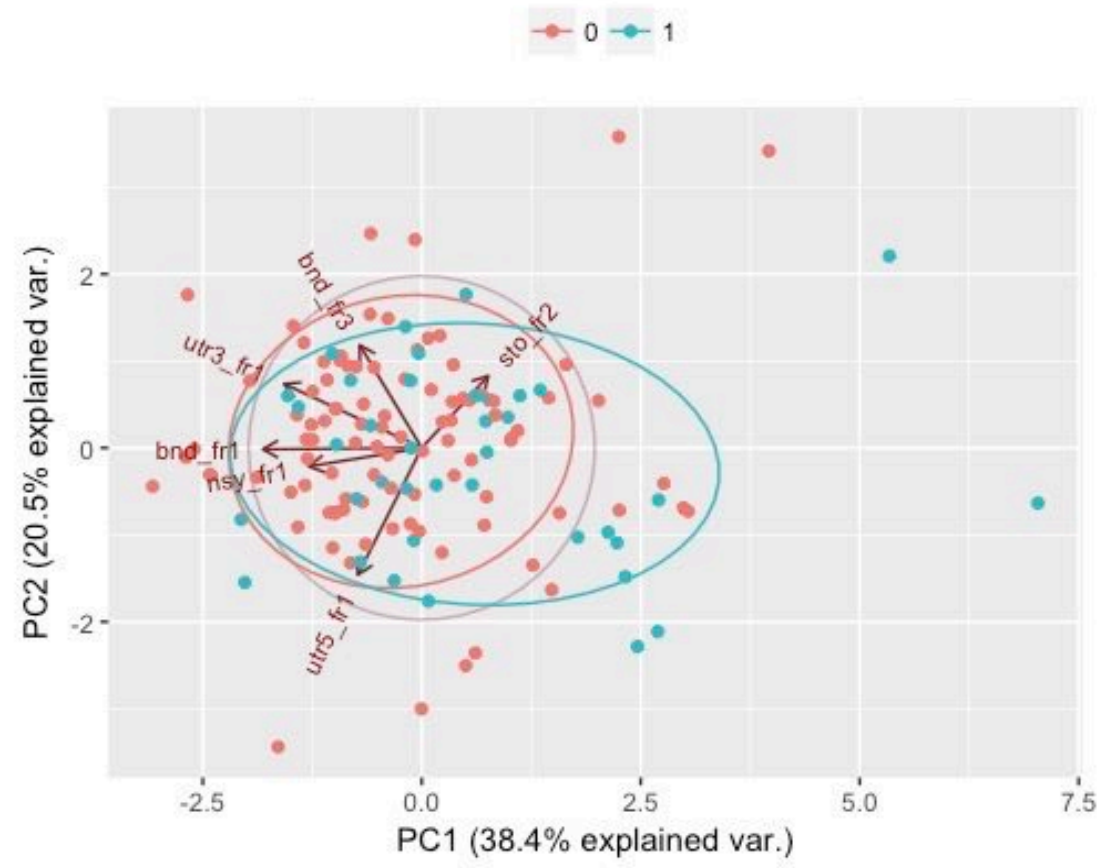

Figure 49: First two PC for the variants bnd_fr1, utr5_fr1, syn_fr1, nsy_fr1, bnd_fr3, utr3_fr1 and sto_fr2 in individuals within cluster 1. Blue represents the ASD group and red represents the control group.

\subsubsection{Differentially expressed genes M18}

Only nfr_fr1 showed a significantly higher mean in the ASD group and was significantly different between the clusters.

Figure 50 shows more control individuals with no nfr_fr1 variants compared to the ASD individuals. There are 54 control individuals with no nfr_fr1 variant, which means $45 \%$ of the total individuals against 12 ASD individuals, making 23\%, with PX2 $\mathrm{p}=0.01$. 


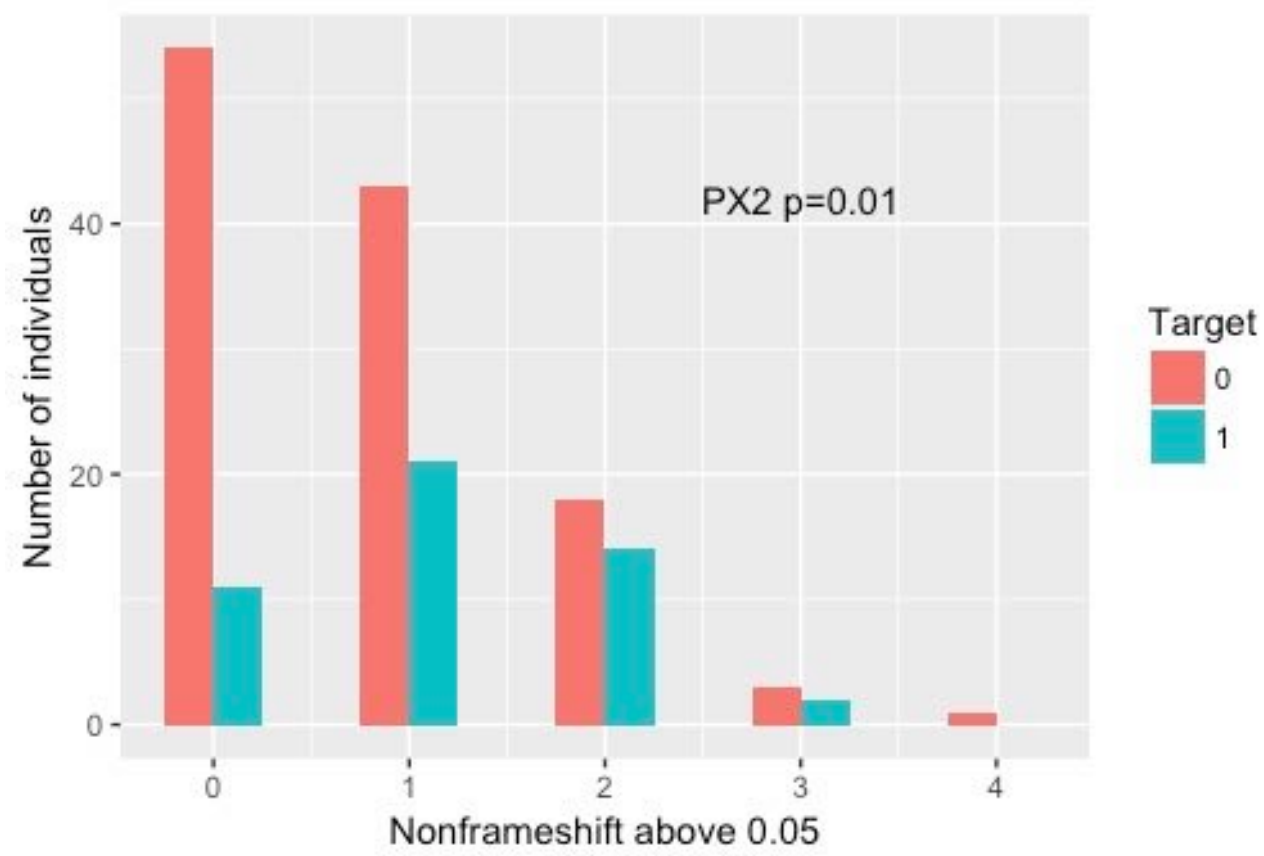

Figure 50: Histogram of Nonframeshift variants with frequency above 0.05 for both GOI. Targets 0 and 1 refer to control and to ASD groups, respectively.

The support vector machine for cluster 2 was a good discriminant approach with AUC above 0.8, KS and $\mathrm{H}$ above 0.4. Nevertheless, the threshold was inverted, and the ASD individuals received smaller numbers of probability than the control group. As a result of this pattern, this outcome was not considered consistent with a discrimination model created with the learning sample.

\subsubsection{RAS/MAPK Pathway}

The variants that revealed a significant difference between affected and control groups were nsy_fr3 with a lower mean for the ASD group.

Since the control group is formed by several individuals with Noonan syndrome, it is expected that they present more rare nonsynonymous variants in RAS/MAPK pathway than the ASD group. After removing the patients with clinical diagnosis of Noonan syndrome, cardiofaciocutaneous syndrome and neurofibromatosis, the lower mean for the ASD group remains unchanged (Wilcoxon $\mathrm{p}=0.007)$.

This could be an indication that this pattern is actually a characteristic of the ASD individuals, and the distribution of these variants between the two groups is seen on Figure 51. It is possible to see that both groups present quite similar distribution, and only the control group presents a normal distribution (Shapiro-Wilk $\mathrm{p}=0.51$ ), ASD (Shapiro-Wilk $\mathrm{p}=0.002$ ). 


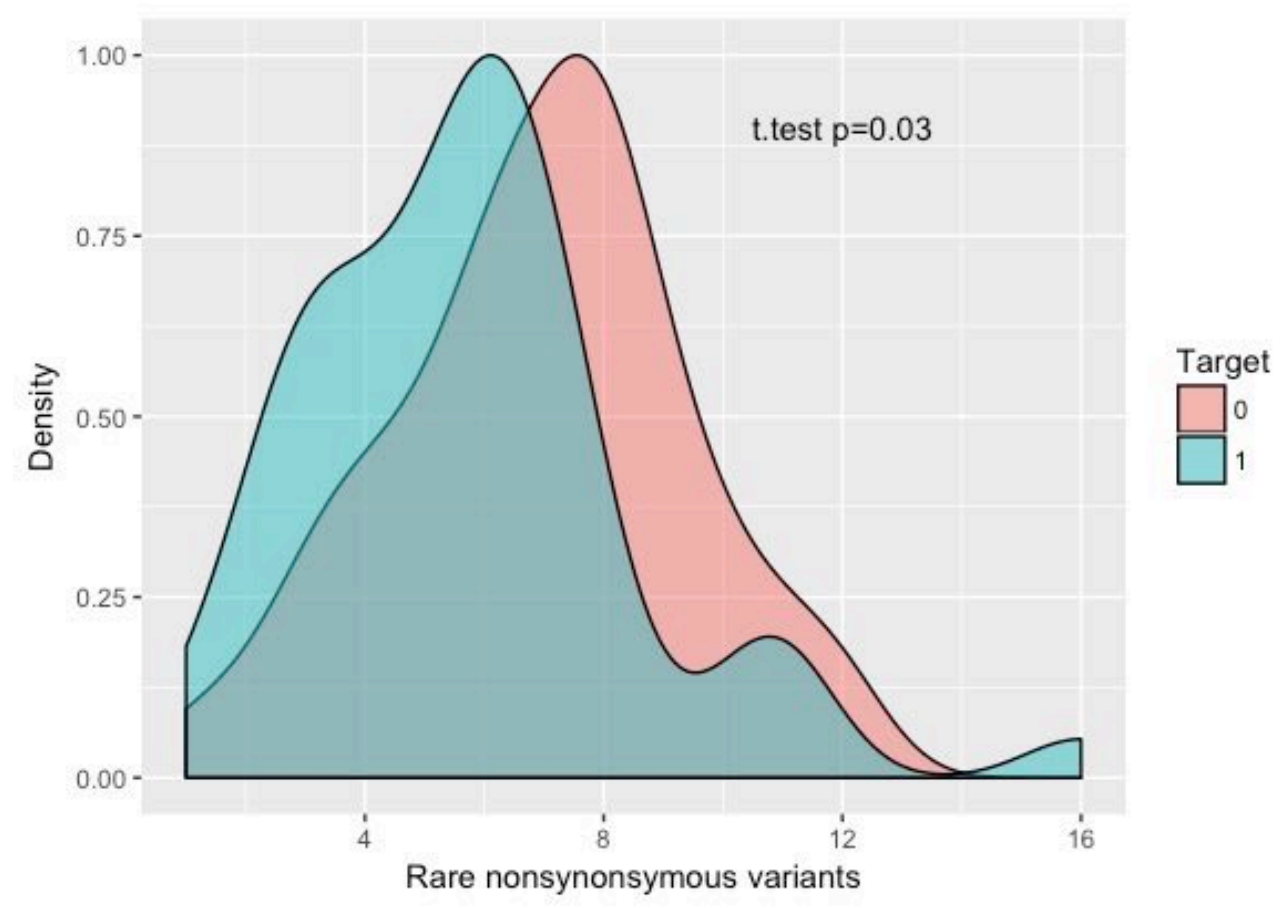

Figure 51: The distribution of the rare nonsynonymous variants between the ASD and control groups without including the individuals with Rasopathies.

The neural network for cluster 2 was a good discriminant approach with AUC close to $0.8, \mathrm{KS}$ and $\mathrm{H}$ above 0.4 . With a threshold of 0.2 , level of sensitivity and level of specificity at $80 \%$. Figure 52 shows the distribution probabilities for both groups, and, in this case, the ASD group tended to have higher probability scores than that of the control group, which can be considered a good model to be further tested with more individuals.

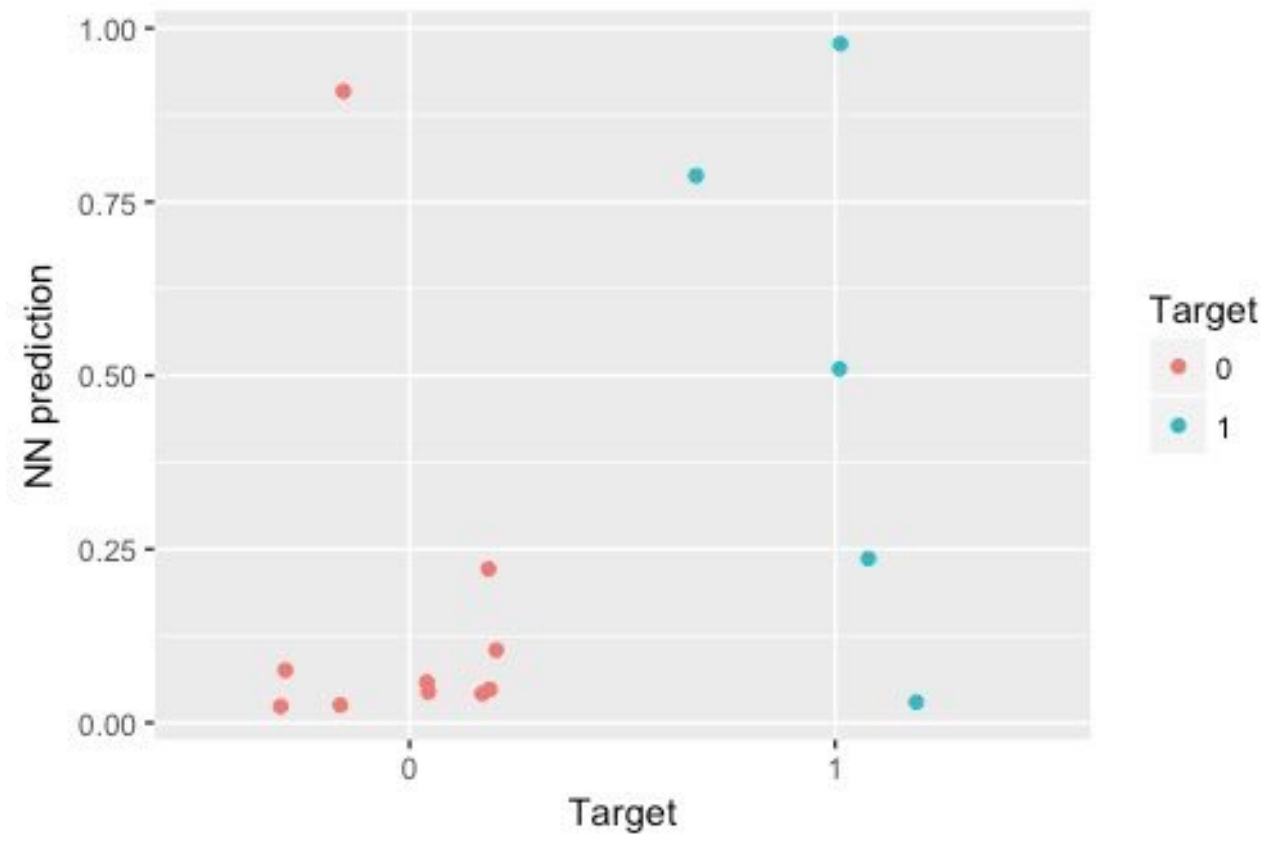


Figure 52: The NN predicted probability for the GOI, where blue represents the ASD group and red represents the control group.

\subsubsection{SFARI High Confidence}

The variants that revealed a significant difference between affected and control groups were fra_fr1, with a higher mean in the ASD group.

$19 \%$ of affected individuals presented one mutation against only $6 \%$ in the control group (PX2 p=0.02).

\subsubsection{SFARI Strong Candidate}

No analysis presented a significant result for this gene list.

\subsubsection{SFARI Suggestive Evidence}

The variants that revealed a significant difference between affected and control groups were fra_fr1 and syn_fr2. The syn_fr2 variant showed a lower mean and the fra_fr1 variant showed a higher mean in the ASD group. None of them presented differences in the cluster comparison.

The distribution of the synonymous variants between $1-5 \%$ of population frequency are very similar, with the complete inclusion of the ASD distribution in the control group. This pattern does not allow a good discriminant model from these variants.

Despite the marginally significant difference between the quantity of common frameshift variants for the groups of interest, both of them presented very similar distribution, with no significant difference when comparing contingency tables for different quantity of variants (PX2 $\mathrm{p}=0.13)$.

\subsubsection{SFARI Syndromic}

The variants that revealed a significant difference between affected and control groups were sto_fr1 and nfr_fr2. The sto_fr1 showed a lower mean in the ASD group 
and nfr_fr2 showed a higher mean in the same group. None of them were different in the cluster comparison.

The common stopgain/loss variants have a similar distribution between the groups but with a significant difference between the individuals bearing one or more variants, $68 \%$ of the control group has at least one variant, while $56 \%$ of the ASD group met the same condition (PX2 $\mathrm{p}=0.006)$.

For the nonframeshift between 1-5\% of population frequency had a marginally significant difference between the groups with $18 \%$ of the control group with one or more variants against $33 \%$ of the ASD group (PX2 $\mathrm{p}=0.045)$.

\subsubsection{SFARI High Confidence, Strong Evidence, Suggestive Evidence and Syndromic (SFARI 3S)}

The variants that revealed a significant difference between affected and control groups were fra_fr1, with a higher mean for the ASD group.

$81 \%$ of the ASD individuals had two or more fra_fr1 variants against $62 \%$ of the control individuals, with a PX2 p=0.019. 


\section{Discussion and Conclusions}

The primary goal of the reports was to detect good models to discriminate two groups of interest using next generation sequencing results. The proposed tests are more suitable for complex diseases compatible with additive model and burden effects.

The first three report analyses can be seen as a proxy of burden tests, and focus on discovering properties directly from variant observation by: (1) comparing each type of variant to investigate a direct contribution to the disease, (2) comparing the role of loss-of-function (LoF) variants, and (3) whether missense variants with higher pathogenic scores by SPRING could be discriminative. With these analyses, it was possible to find direct reasons that confer differences between the groups and to elaborate physiologic models for further testing. As an example, in a scenario of a very rare, severe, autosomal dominant full penetrant genetic condition caused by gain-offunction mutations, like Thanatophoric Dysplasia, Type I, a higher number of individuals is expected to present singleton likely gene-disruptive missense mutations in candidate genes in the affected group than in the control group. This signal should be seen in the analysis of singleton missense variants ranked by SPRING with different peaks on the q-value distribution curve, a peak with lower q-values for the control group and one with higher q-values for the affected group. This type of analysis, with different approaches, is part of most of the current whole exome sequencing (WES) procedures in genomic diagnosis laboratories, which use a gene list based on the phenotype of the subject under investigation and search for highly disruptive variants (LoF, missense highly damaging and extremely rare variants) that could explain the development of the condition (TRUJILLANO et al., 2016).

In turn, the multivariate approaches (logistic regression, decision tree, neural network, support vector machine and principal components) are attempts at understanding the interactions among different types of variant to explain complex diseases (ZHAO; PAN; DU, 2018). As an example of a disease with an oligogenic mode of inheritance, in order to develop the affected phenotype, two phenomena must happen: first, the individual has to lack common variants, which normally confer protection against the development of the disease, and, secondly, a rare LoF mutation in any gene of the same pathway. In this scenario, for the affected group, a lower number of common variants with a higher number of rare LoF compared to that of the control group should be expected. The characteristic that differs from the first scenario is that both events should happen together in order to develop the phenotype, since the analysis of common or rare LoF variants alone would not be sufficient to differentiate the groups of interest. Multivariate approaches could suggest more complex relationships.

The 21 reports aimed at testing these two scenarios with different gene lists that were previously described in the literature as related to autism spectrum disorder (ASD), making it clear that the main goal of the reports was to create models based on the knowledge already produced to distinguish affected from control groups and not to propose new pathways to the disease. It is mandatory to understand the difference between discovering candidate genes or new pathways from determining which 
model can be applied for the diagnosis of a particular individual.

Many different methodologies were developed to understand the genetic background of ASD, and the most present in literature is the burden analysis, which investigates what type of variant is the most representative among individuals with ASD. Based on this type of investigation, many conclusions were already presented in the introduction, including the role of de novo and rare inherited likely gene-disruptive mutations (IOSSIFOV et al., 2012; KRUMM et al., 2015; SANDERS et al., 2012).

Efforts were made toward developing integrated models to explain ASD genetics. The Transmission And De novo Association, known as TADA, is one of those largely used models, and this approach integrates the information from de novo and inherited variants in affected and control groups. With this method, it is possible to access candidate genes that confer higher risk for developing ASD, being more inclusive than a simple comparison for the presence of de novo LoF (HE et al., 2013). This method was used by Li et al. (2018) in the context of discovering different genes and pathways for the ASD subcategories and suggested that the three ASD subcategories (Asperger syndrome, autism and pervasive developmental disorder not otherwise specified) have more convergences than divergences concerning the type of genes and mutations related to the different phenotypes (LI et al., 2018). Liu et al. (2014) developed a method, called DAWN, that uses TADA scores to identify candidate genes in networks based in the co-expression in mid-fetal prefrontal and motor somatosensory neocortex (LIU et al., 2014). Luo et al. (2018) developed a multilevel genetic prioritization and association analysis to construct a better model for the development of the disease.

The importance of deciding to use the statistical approach in the investigation of common complex diseases is well illustrated by the publications by Stessman et al. 2017 and Barret et al. 2017. Stessman et al. 2017 published an article in which they described 91 neurodevelopment risk genes from 208 candidate genes from the literature and sequenced more than 10,000 affected individuals with a broad spectrum of neurodevelopment disorders. They conducted a simulation by shuffling the labels of private case and control observations 100,000 times and calculated the probability of observing at least the number of likely gene-disruptive (LoF or missense variants with CADD prediction over 30) events seen among the cases. These P values were corrected (Benjamini-Hochberg) to the number of genes in the study $(n=208)$ (STESSMAN et al., 2017). Barret et al. 2017 draw attention to the statistical challenges for studies involving gene finding and confirmatory hypothesis of complex diseases, such as ASD, by replying to the Stessman et al. 2017 publication. Barret's article described the possible statistical flaws that could lead to a misconception of the results. The main statistical issue was the threshold applied to the $\mathrm{p}$-value that did not correct the effects of scanning all 20,000 genes, and despite the significance of the replication sample, the rate of de novo LoF mutations was dramatically lower (BARRETT et al., 2017), thus showing the importance of determining the statistical methodology very carefully. Also, the work raises the importance of having population matched controls, sequencing and analysis in identical ways and the average depth of coverage for the target regions. Without setting these parameters correctly, even the use of permutation test to assess significance does nothing toward 
eliminating these issues.

Until now, all the knowledge acquired about pathophysiology has not been applied to the clinical-laboratory context to test whether a stepwise and statistical approach to analyze WES of probands without parents sequencing information could result in an efficient discrimination of individuals and bring information about the genetic development of the phenotype to that specific subject. The main goal of the reports was to test whether the hypothesis for developing ASD could generate good models that could be applied as a routine test within the clinical-laboratory context. Regarding this context, all but two reports returned a preliminary result that could be further investigated concerning the strength of the models. These results and implications for the reality of future WES testing will be discussed later.

With the exception of the GABA pathway and SFARI Strong Candidate, all of the reports resulted (19 of 21) in at least one significant difference between the groups that could be further investigated. From these 19 reports, the analysis with the highest number of positives was the Comparison for each variant between affected and control groups, and this is in accordance with the burden model for developing ASD. However, many significant results were concentrated in variants that were not considered likely gene-disruptive, such as common or synonymous, and a higher number of mutations in the control groups, such as rare missense RAS/MAPK variants. These findings were not expected based on most of ASD candidate genes publications, which have prioritized loss-of-function mutations (LA TORRE-UBIETA et al., 2016; WOODBURY-SMITH; SCHERER, 2018).

The Comparison for each variant between affected and control groups was significant for 19 reports, eleven of them maintained at least one variant with significant difference after further investigation, and eight presented variants with higher mean in ASD groups [chromatin target, 208 genes, hypermutable, differentially expressed M2, M8 and M18, SFARI High Confidence (SFARI1) and SFARI all (SFARIS3)], one with both higher and lower mean in the ASD group [SFARI Syndromic (SFARIS)] and two with only lower mean in the ASD group (mTOR and RAS/MAPK). The sole analysis of the level of significance of each finding is not the best approach to eliminate or consider a specific scenario, while the investigation of the distribution and the critical interpretation of physiological and genetic pathways is fundamental for making the final decision to pursuit a theory and confirming other scenarios.

The gene lists, mTOR, SFARI Syndromic (SFARIS) and RAS/MAPK, had variants that showed a significant decrease in number in the ASD group. The nonsynonymous rare variants from the RAS/MAPK group presented different distributions in the ASD and control groups, with only the first showing a normal distribution, and this pattern remained unchanged even after the individuals diagnosed with rasopathies were removed. Even with a marginal p-value (0.03), this result could suggest a certain protective effect of the missense rare mutations in this pathway that remained unchanged after removing the individuals. The mTOR gene list also had a marginally significant ( $p$-value $=0.014$ ) decrease in missense mutations for the ASD group but not in rare variants, instead for the ones with frequency between 0.01 and 0.05 , suggesting also a protective role for the missense mutations in 
different pathways. The exploratory analysis without gene filtering also revealed a significant lower mean in the ASD group for this type of variant, and, for that reason, this is still a preliminary result that requires further testing, specially to detect whether this is not a biased result by the set of individuals in this analysis. Nevertheless, this specific protective effect of missense variants was not described in the literature until now and could be a promising model to pursue with more individuals and test in different samples.

The SFARIS genes and chromatin modifier presented somewhat conflicting results, since the first showed a significant decrease in the common stopgain variants in ASD, while the latter showed an increase, with special attention to the fact that the chromatin result, in addition to singleton splicing mutations, is the most significative of all reports. The SFARIS genes consists, in most cases, of fully penetrant recognizable syndromes that follow the LoF model and was the only list that had both lower and higher mean variants for the ASD group with significant results. The common nonframeshift mutations had a higher mean in the ASD group, with $33 \%$ of the individuals with one or more variants versus $18 \%$ from the control group. One hypothesis that could be pursued with these results is to test in a larger cohort a combined model of inheritance that modifies the phenotypic outcome for the patients. However, since this group of genes belongs to very rare clinically recognizable diseases, further investigation to test multivariate models with common mutations may not be the best approach.

In turn, the chromatin genes with an increase of common nonsense and singleton canonical splicing variants could reveal a combination of events that predispose to ASD, and therefore should be further investigated. Publications about chromatin modifier genes focused mainly on rare or de novo highly gene-disruptive variants (COTNEY et al., 2015; DE RUBEIS et al., 2014; GALLAGHER et al., 2014; O'ROAK et al., 2012b). The finding that common nonsense variants could be acting together with highly rare canonical splicing events is in accordance with the model proposed by Luo et al. 2018 with one gene hit and a second pathway hit. Since these are preliminary results, further testing is needed to confirm this finding in a larger cohort, and test the second hit model for these genes.

Besides the chromatin modifiers, other gene lists showed a higher number of variants in the ASD group, such as: 208 genes, hypermutable, differentially expressed M2, M8 and M18, SFARI1 and SFARIS3. For the other lists, different variants were responsible for the findings. The descriptions above highlighted the most prominent results that should be first addressed, but each of these variants could set off investigations in larger cohorts.

The role of machine learning predictive models in medicine is getting larger for areas such as imaging and cancer treatment (GREENLAW et al., 2017; LETAI, 2017; MACHIELA et al., 2011), and other aspects of ASD diagnostics as well, such as electroencephalogram, movement patterns and brain imaging (BOSL; TAGERFLUSBERG; NELSON, 2018; LI et al., 2017; ZARE; REZVANI; BENASICH, 2016), thus, the application of predictive models was an approach that was considered for all gene lists. No logistic regression, neural network, support vector machine or decision tree 
returned consistent good discrimination models. This result was not expected considering the arising models of multiple causes for the development of the ASD phenotype (BETANCUR; SAKURAI; BUXBAUM, 2009; LUO et al., 2018; PINTO et al., 2014). These approaches were chosen because they consist of different strategies that can use quantitative variables to construct discrimination models and differentiate the groups by finding one of the possible resolutions, and they are not always interpreted straightforwardly. All of them depend on learning the patterns from individuals seen as examples of discriminant behavior in search for a pattern that best separates them (ZHAO; PAN; DU, 2018).

Differently from the multivariate approaches described above, the principal component analysis is not based on learning and testing sets to construct a discrimination model; instead, it divides the individuals in a graphical space based on the variance within the variables, revealing which variables are most related to the difference among groups (ASCHARD et al., 2014). This approach was chosen because it can evaluate all the data available, without the bias of having to choose different sets of individuals. So, it was tested in two different ways, one with the counting matrix as a proxy of the additive model, and the other with the raw VCF, so that the variants alone and their frequency only in the cohort could be tested for patterns, and not related to their effect on population or protein. If a set of specific variants was responsible for the disease, it would be seen in this approach. None of the raw VCF principal components analysis resulted in a specific variant or sets of variants that could be discriminated between the groups. This finding is in accordance with a recent systematic reconstruction of the ASD genetic profiles that revealed significant recurrence only for genes and pathways, but not for specific variants (LUO; SOROCK, 2008).

All the multivariate approaches are susceptible to type and quantity of variables, and, accordingly, it is always hard to choose between gaining and losing power with the addition of variables. In this pipeline, many possible variants are taken into account, and when analyzed together, particularly with a small number of individuals, the signal of good discrimination models could be lost (HASTIE; TIBSHIRANI; FRIEDMAN, 2013). In order to determine the best set of variants, all possible combinations should be tested, a computationally exhaustive scenario, and, to address this issue, we chose to decrease the number of variables by separating them into two groups, even with the risk of losing the relations among them: (1) variants that affect protein directly and (2) variants in regulatory regions.

Other important matter to address when dealing with complex diseases is the heterogeneity of the groups (BETANCUR, 2011; BORA et al., 2016), since, in addition to the direct analysis of affected and control groups, the separation into clusters based on the final counting matrix adds power to the discovery process by two main paths. The first path is to detect major differences in the sequencing process and separate the groups to avoid bias, an example of what has been found in this study. The second path is related to the heterogeneity of distinct diagnosis for complex diseases, where the goal was to separate the individuals into more homogeneous groups (clusters), which could represent individuals with similar backgrounds for the development of the disease. 
In general, none of the multivariate approaches returned good models for further testing. The presence of significant results for the multivariate approaches only in cluster 2 draw attention to an issue concerning this type of investigation. Two main scenarios could explain this pattern: if the individuals in the cluster were separated because of intrinsic sequencing issues, and not related to their direct genetic background, which seems to be the case in this study, then this result can be the consequence of a particular sample separation, and not an indication of the genetic structure of the disease. The second scenario is that, if the individuals were separated by their genetic architecture, the presence of such a signal in only one architecture indicates that not all individuals should be analyzed equally, even if the phenotype is the same.

Other contribution of this study to ASD research was to identify that individuals, sequenced in different time lapses and with data obtained from different bioinformatics pipeline, can harbor significant differences that cannot be removed during the investigation process and are not evident with classical quality assessments, such as number of variants per individual, transition/transversion proportions and other metrics. The choice of the individuals that will form the affected and control groups have to be very carefully made, and the interpretation of the results with database limitations in mind along with confirming the findings in different sets could lead to a more reliable conclusion. Research in the ASD field is very common, and every time new hypotheses are raised and tested, this consequent large number of publications, from many regions of the world, sometimes confirms an important finding, such as the FMRP target and CHD8 involvement in the disease (BARNARD; POMAVILLE; O'ROAK, 2015; COTNEY et al., 2015; DARNELL et al., 2011; STEINBERG; WEBBER, 2013). But sometimes, it reveals largely different candidate genes, with the exclusion of important ones, such as SHANK3 and PTEN (CUKIER et al., 2014; HE et al., 2013; KRISHNAN et al., 2016; WOODBURY-SMITH et al., 2015).

This study was very effective in showing that, even with the limitations to the number of individuals, many different results were obtained from a heterogeneous, relatively common complex disease. Most of the gene lists had at least one significant result, and $p$-values varied from borderline $(p=0.045)$ to very low $(p=9 e-05)$. But none of these results alone should be considered proof enough of a specific contribution to the pathophysiology of the disease, instead testing the hypothesis in other samples with matched controls, analyzing the distribution of the results and how the statistics counts the p-values, and doing further testing, such as cellular and animal models, and looking into whether similar results could be obtained from different candidate genes (BARNARD; POMAVILLE; O'ROAK, 2015; IOSSIFOV et al., 2015; KRISHNAN et al., 2016). This could be some of the approaches that would make a more reliable final result concerning ASD.

All the reports include many statistical tests which should be corrected for multiple testing, thus the level of significance for each result should be accounted carefully. The choice of not making the correction already on the report was made because the level of significance accepted could change for each research with the chance of losing interesting relations that could be confirmed otherwise. 
One approach that can be proposed to improve the statistical confidence of the results of ASD research (BARRETT et al., 2017) is to divide the sample into learning and testing (even if the main strategy does not involve machine learning). With the learning samples, all the research protocol is followed, then the testing samples are resampled into the necessary number of smaller sets, depending on the strength of the initial finding. The sets should follow three configurations, (1) individuals classified a priori as affected and control, (2) individuals classified a priori as control, but randomly separated in affected and control, and (3) individuals classified as affected, but randomly separated as affected and control. The protocol of the principal hypothesis is made in each sample and the proportion in which the same result appears will be relevant to the importance of this model to the disease. With this strategy it is possible to confirm a finding as a general characteristic of the disease, or a particular characteristic of some individuals with the disease, or a characteristic that could be found for the population (with the results from the two homogeneous groups). This approach is consistent with the one applied by Iossifov et al. 2015, in which, in order to test for burden of rare and de novo mutations in ASD, they performed a permutation by swapping genes between sets and permuting the "affected" labels (IOSSIFOV et al., 2015).

Other important limitation to our study was that only specific gene lists could be accessed for the tests, regardless of the pipeline at hand and further knowledge of the necessary steps to develop the disease. In studies such as that by Luo et al. 2018 that showed that most variants disrupt extremely close residues within the same gene, or affect similar pathway-related gene functions, or that by Uddin et al. 2014, that showed a higher concentration of variants in brain expressed exons (LUO; SOROCK, 2008; UDDIN et al., 2014), a second layer of interpretation can be added to the variants and provide a more reliable model.

The final annotated file from a WES has around 50,000 variants per individual and its analysis consists mainly in experts prioritizing variants based on clinical features, population frequency, in silico predictor and scientific literature (EILBECK; QUINLAN; YANDELL, 2017). Some parts of the WES analysis can be automatized, such as filtering for variant quality, population frequency, and in some cases, phenotype (MUGHAL et al., 2017; SOBREIRA et al., 2015). Even with these modifications, the initial parameters have to be set by experts and the remaining variants are also classified and analyzed by them. For mendelian disorders, these steps increase the chance of finding the variant related to the phenotype described (EILBECK; QUINLAN; YANDELL, 2017).

The addition of WES in the clinical laboratory practice as a manner for diagnosing rare genetic diseases was well accepted due to its high diagnostic yield (around 25-30\%), and lower final investigation cost (TRUJILLANO et al., 2016). For genetic diseases with a clear mendelian inheritance, the advantages of WES are very straightforward, but for complex diseases, such as ASD, there are remaining questions about whether or not to use this approach for molecular diagnosis purpose (RETTERER et al., 2016).

The importance of differentiating the clinical report from the scientific literature 
is that, for the last one, significant burden results add to understanding the disease's global etiology, it is not made to change particular medical decisions and to confirm the diagnosis, while laboratory results will be seen by doctors and family members as a result for the specific patient (MATULLO; DI GAETANO; GUARRERA, 2013). Even with the advances of metabolomics, biochemistry, brain imaging and electroencephalography, there are no clear biomarkers to distinguish the ASD group from the control group that can be used in the clinical practice or that can be related to an individualized therapy (DIEME et al., 2015; HAMPTON, 2017; LA TORREUBIETA et al., 2016). Thus, the models could still be tested within a context of more samples.

ASD is a complex disease and, although its pathophysiology is clearer, thousands of genes and mutations are described, making it hard to select the few disease-causing events and to replicate it in other studies (LUO; SOROCK, 2008). In most cases, ASD does not follow the mendelian inheritance, and that is the reason why the American College of Medical Genetics and Genomics (ACMG), followed by other institutions, does not indicate WES as one of the genetic strategies for molecular diagnosis, but recognizes that, in the future, this could bring more evidence to the diagnosis (SCHAEFER; MENDELSOHN, 2013).

Although the institutions do not formally recommend the application of WES testing to ASD, the physicians request this test in the hope that the patient presents a pathogenic variant that could explain the phenotype, and that it could aid further investigations and genetic counseling. This demand associated with scientific reports that show an increase in rare or de novo loss-of-function (LoF) gene variants that are strongly related to autism (such as genes of the SFARI High Confidence, or other lists, many of them tested in this study) (COTNEY et al., 2015; JIANG et al., 2013; LA TORRE-UBIETA et al., 2016; SANDERS et al., 2012) are leading WES analysis to search for highly gene-disruptive variants in such genes.

All things considered, this study reached its goal of developing a pipeline to investigate different approaches to discriminating individuals from a complex disease, but none of the 21 different gene lists revealed any positive result that could be used in the clinical practice without being further investigated. The preliminary results suggest that different types of variants can have distinct degrees of contribution, depending on the pathway or genes analyzed and that the different contribution to each variant is more prominent than the role different variants play in the same pathway or genes, represented by the presence of significant results in the variant analysis alone versus nonsignificant results in the multivariate approaches. Nevertheless, this work's primary achievement was to reveal the weakness for the main assumptions when analyzing WES for ASD within the clinical context, concluding that the institutions should be more careful about the possibility of having false positives, and, for now, remaining concentrated on the ASD mendelian syndromes with LoF de novo variants in fully penetrant genes. 


\section{Resumo}

O transtorno do espectro autista (TEA) é um distúrbio do neurodesenvolvimento caracterizado por uma incapacidade de comunicação comportamento e interações sociais que afeta em torno de 1-2\% da população mundial. Até o momento a etiologia do TEA ainda não é totalmente compreendida, mas nos últimos 18 anos muitos avanços foram feitos para entender o componente genético relacionado ao desenvolvimento do quadro clínico. Com o advento das análises de varredura genômica como a análise cromossômica por microarray e o sequenciamento completo de exoma (SCE) muitos avanços foram feitos para a compreensão da fisiopatologia da doença. Em torno de 10-15\% dos casos podem ser explicados por grandes perdas ou ganhos (deleções ou duplicações superiores a 1000 pares de bases) do material genético, que geralmente envolvem a disrupção de um ou mais genes. As metodologias de sequenciamento de nova geração foram fundamentais para a descrição das mutações de ponto e pequenas inserções e deleções associadas ao TEA. O SCE permitiu que muitas descobertas fossem feitas sobre novos genes candidatos e mecanismos para o desenvolvimento da doença. Atualmente afirma-se que as alterações de novo (não herdadas) e de maior probabilidade de ruptura gênica, como as mutações de perda-de-função e as alterações não-sinônimas com alta predição de dano por ferramentas computacionais, em genes de susceptibilidade a doenças do neurodesenvolvimento sejam um grande contribuidor para o mecanismo da doença. Entretanto essas mutações, além de não explicar a totalidade dos casos raramente são recorrentes na população, o que dificulta $\mathrm{o}$ estabelecimento de um diagnóstico molecular definitivo para a maioria dos pacientes. O SCE já é uma prática nos laboratórios clínicos de genética e demonstra uma alta efetividade para as doenças que seguem um padrão de herança mendeliano, e têm uma causa genética estabelecida. Na prática clínica o SCE é solicitado para os casos de TEA, apesar de ter diferentes modos de herança e terem mais de 1,000 genes associados à doença. Devido a estas características o SCE para os casos de TEA são um grande desafio para o laboratório clínico. Este estudo propõem a construção de uma rotina computacional de análise do SCE que possa testar diferentes genes candidatos quanto à sua sensibilidade e especificidade para a detecção dos indivíduos afetados. A abordagem proposta é a contagem de variantes separadas por seu possível dano à proteína e frequência populacional para cada indivíduo de grupos afetado e controle em 168 indivíduos com SCE, sendo 49 com TEA e 119 controles. Após a formulação da contagem esses valores são submetidos a uma sequência de testes estatísticos, buscando diferença significativa em quantidade de mutações de todas as variantes isoladamente, das mutações de perda-de-função, ou não-sinônimas danosas como um conjunto e a aplicação de modelos de análise multivariada como: regressão logística, árvore de decisão, rede neural, máquinas de suporte de vetor e análise de componente principal para a elaboração de modelos mais complexos para o desenvolvimento na doença. Ao todo foram testadas 21 listas de genes, destas, 19 apresentaram ao menos um resultado significativo, sendo a análise de variantes isoladamente a que obteve maior número de eventos significativos. Desde variantes aparentemente protetoras (maior número no grupo controle), como as variantes não-sinônimas em via de RAS/MAPK quanto variantes de perda de códon de parada com frequência 
populacional acima de 0.05 em genes de cromatina em maior número nos indivíduos com TEA. Nenhum dos modelos de análise multivariada obteve resultados significativos na discriminação entre os dois grupos. Devido ao pequeno número amostral os resultados deste estudo devem ser interpretados com limitações, sendo necessária a replicação deste cenário em outros bancos de dados. Entretanto, estes achados sugerem que diferentes tipos e frequências de variantes podem ter contribuições distintas para o desenvolvimento da doença a depender dos genes analisados, mais de que relações complexas entre as variantes de uma mesma lista de genes. 


\section{Abstract}

Autism spectrum disorder (ASD) is a neurodevelopment disorder characterized by impairment in communication skills, behavior and social interaction that affects roughly 1-2\% of individuals worldwide. To date, ASD etiology has yet to be fully understood, but in the past 18 years many advances have been made toward understanding the genetic component related to the development of the clinical phenotype. With the advent of genomic scan analyses, such as chromosome analysis by microarray and whole exome sequencing (WES), many advances have been made toward understanding the disease's pathophysiology. About $10-15 \%$ of the cases can be explained by large losses or gains (deletions or duplications greater than 1000 base pairs) in genetic material, which generally involve the disruption of one or more genes. Next generation sequencing methodologies were essential for describing point mutations and small insertions and deletions associated with ASD. WES has allowed many discoveries to be made regarding new candidate genes and mechanisms for the development of the disease. It is now claimed that de novo (non-inherited) and likely gene-disruptive mutations, such as loss-of-function and non-synonymous changes with high prediction of damage by computational tools, in neurodevelopmental genes are major contributors to the disease mechanism. However, these mutations, in addition to not explaining the majority of cases, are rarely recurrent in the population, thus making it difficult to establish a definitive molecular diagnosis for most patients. WES is already a practice in clinical genetics laboratories and demonstrates high effectiveness for diseases that follow a Mendelian inheritance pattern and have an established genetic cause. In the clinical practice, WES is requested for ASD cases despite the different inheritance modes and more than 1,000 genes associated with the disease. As a result of these characteristics, the WES analysis for ASD is a major challenge for the clinical laboratory. This study proposes the creation of a computerized WES analysis routine that can test different candidate genes for their levels of sensitivity and of specificity for detecting affected individuals. The proposed approach consists of counting the variants sorted by their possible protein damage and population frequency for each individual from affected and control groups. This study conducted 168 WES analyses, 49 of them with ASD individuals and 119 of them with control individuals. After counting formulation, these values are subjected to a sequence of statistical tests, seeking to find a significant difference in the amount of mutations of all the variants alone, loss-of-function or damaging missense mutations, and to the application of models of multivariate analysis, such as: logistic regression, decision tree, neural network, vector support machine and principal component analysis for creating more complex models for disease development. A total of 21 gene lists were tested, out of which 19 presented at least one significant result, and the analysis of variants alone was the one that obtained the largest number of significant events. From apparently protective variants (higher number in the control group), such as the missense variants in RAS/MAPK pathway as variants of stopgain with population frequency above 0.05 in chromatin genes in greater number in individuals with ASD. None of the multivariate analysis models presented significant discrimination results between the two groups. Due to the small sample size, the results of this study should be interpreted with limitations, and these scenarios need 
to be replicated in other databases. However, these findings suggest that different types and frequencies of variants may have distinct contributions to the development of the disease depending on the genes analyzed, rather than complex relationships between variants on the same gene list. 


\section{Reference}

ABRAHAMS, B. S.; GESCHWIND, D. H. Advances in autism genetics: on the threshold of a new neurobiology. Nature Reviews Genetics, v. 9, n. 5, p. 341-355, maio 2008.

AKAIKE, H. Prediction and Entropy. In: A Celebration of Statistics. Traducao. New York, NY: Springer, New York, NY, 1985. p. 1-24.

ANAGNOSTOPOULOS, C.; HAND, D. J. hmeasure: The H-measure and other scalar classification performance metrics. 2012.

ANAGNOSTOU, E. et al. Autism spectrum disorder: advances in evidence-based practice. CMAJ : Canadian Medical Association journal = journal de l'Association medicale canadienne, v. 186, n. 7, p. 509-519, 15 abr. 2014.

ANASTASIADIS, A. D.; MAGOULAS, G. D.; VRAHATIS, M. N. New globally convergent training scheme based on the resilient propagation algorithm.

Neurocomputing, v. 64, p. 253-270, 1 mar. 2005.

ASCHARD, H. et al. Maximizing the Power of Principal-Component Analysis of Correlated Phenotypes in Genome-wide Association Studies. The American Journal of Human Genetics, v. 94, n. 5, p. 662-676, 5 jan. 2014.

AZIZ, N. et al. College of American Pathologists' laboratory standards for nextgeneration sequencing clinical tests. Archives of pathology \& laboratory medicine, v. 139, n. 4, p. 481-493, abr. 2015.

BARNARD, R. A.; POMAVILLE, M. B.; O'ROAK, B. J. Mutations and Modeling of the Chromatin Remodeler CHD8 Define an Emerging Autism Etiology. Frontiers in Neuroscience, v. 9, 2015.

BARRETO, A. S. MODELOS DE REGRESSAO. Traducao. [s.l: s.n.].

BARRETT, J. C. et al. New mutations, old statistical challenges. bioRxiv, p. 115964, 12 mar. 2017.

BAXTER, A. J. et al. The epidemiology and global burden of autism spectrum disorders. Psychological Medicine, p. 1-13, 11 ago. 2014.

BERTOLA, D. R. et al. Further evidence of the importance of RIT1 in Noonan syndrome. American Journal of Medical Genetics Part A, p. n/a-n/a, 1 ago. 2014.

BETANCUR, C. Etiological heterogeneity in autism spectrum disorders: more than 100 genetic and genomic disorders and still counting. Brain Research, v. 1380, p. 4277, 22 mar. 2011.

BETANCUR, C.; SAKURAI, T.; BUXBAUM, J. D. The emerging role of synaptic celladhesion pathways in the pathogenesis of autism spectrum disorders. Trends in neurosciences, v. 32, n. 7, p. 402-412, jul. 2009. 
BORA, E. et al. Heterogeneity of subclinical autistic traits among parents of children with autism spectrum disorder: Identifying the broader autism phenotype with a data-driven method. Autism research : official journal of the International Society for Autism Research, 1 jul. 2016.

BOSL, W. J.; TAGER-FLUSBERG, H.; NELSON, C. A. EEG Analytics for Early Detection of Autism Spectrum Disorder: A data-driven approach. Scientific Reports, v. 8, n. 1, p. 6828, 1 maio 2018.

BOURGERON, T. From the genetic architecture to synaptic plasticity in autism spectrum disorder. Nature Reviews Neuroscience, v. 16, n. 9, p. 551-563, 1 set. 2015.

BRANDLER, W. M. et al. Frequency and complexity of de novo structural mutation in autism. bioRxiv, p. 030270, 30 out. 2015.

BRANDLER, W. M. et al. Paternally inherited cis-regulatory structural variants are associated with autism. Science, v. 360, n. 6386, p. 327-331, 20 abr. 2018.

BREIMAN, L. Classification and Regression Trees. Traducao. [s.l.] Routledge, 2017.

C YUEN, R. K. et al. Whole genome sequencing resource identifies 18 new candidate genes for autism spectrum disorder. Nature Neuroscience, v. 20, n. 4, p. 602-611, abr. 2017.

CELLOT, G.; CHERUBINI, E. GABAergic Signaling as Therapeutic Target for Autism Spectrum Disorders. Frontiers in Pediatrics, v. 2, 8 jul. 2014.

CENTERS FOR DISEASE CONTROL AND PREVENTION. Prevalence of autism spectrum disorders--Autism and Developmental Disabilities Monitoring Network, 14 sites, United States, 2008. Morbidity and mortality weekly report. Surveillance summaries (Washington, D.C. : 2002), v. 61, n. 3, p. 1-19, 30 mar. 2012.

CHAHROUR, M. H. et al. Whole-Exome Sequencing and Homozygosity Analysis Implicate Depolarization-Regulated Neuronal Genes in Autism. PLoS Genetics, v. 8, n. 4, p. e1002635, 12 abr. 2012.

CHONG, J. X. et al. The Genetic Basis of Mendelian Phenotypes: Discoveries, Challenges, and Opportunities. The American Journal of Human Genetics, v. 97, n. 2, p. 199-215, ago. 2015.

COLVERT, E. et al. Heritability of Autism Spectrum Disorder in a UK PopulationBased Twin Sample. JAMA Psychiatry, v. 72, n. 5, p. 415-423, 1 maio 2015.

COTNEY, J. et al. The autism-associated chromatin modifier CHD8 regulates other autism risk genes during human neurodevelopment. Nature communications, v. 6, p. 6404-6404, 1 jan. 2015.

CUKIER, H. N. et al. Exome sequencing of extended families with autism reveals genes shared across neurodevelopmental and neuropsychiatric disorders. Molecular autism, v. 5, n. 1, p. 1, 2014.

DARNELL, J. C. et al. FMRP Stalls Ribosomal Translocation on mRNAs Linked to Synaptic Function and Autism. Cell, v. 146, n. 2, p. 247-261, 2011. 
DE RUBEIS, S. et al. Synaptic, transcriptional and chromatin genes disrupted in autism. Nature, v. 515, n. 7526, p. 209-215, 13 nov. 2014.

DE RUBEIS, S.; BUXBAUM, J. D. Genetics and genomics of autism spectrum disorder: embracing complexity. Human Molecular Genetics, 2015.

DEVLIN, B.; SCHERER, S. W. Genetic architecture in autism spectrum disorder. Current Opinion in Genetics \& Development, v. 22, n. 3, p. 229-237, jun. 2012.

DIEME, B. et al. Metabolomics study of urine in autism spectrum disorders using a multiplatform analytical methodology. Journal of proteome research, $p$. acs.jproteome.5b00699, 5 nov. 2015.

EILBECK, K.; QUINLAN, A.; YANDELL, M. Settling the score: variant prioritization and Mendelian disease. Nature Reviews Genetics, v. 18, n. 10, p. 599-612, 1 out. 2017.

FAWCETT, T. An introduction to ROC analysis. Pattern Recognition Letters, v. 27, n. 8, p. 861-874, jun. 2006.

FIELD, A. Discovering Statistics Using IBM SPSS Statistics. Traducao. [s.1.] SAGE, 2017.

FOMBONNE, E. Editorial: The rising prevalence of autism. Journal of Child Psychology and Psychiatry, v. 59, n. 7, p. 717-720, 19 jun. 2018.

FRITSCH, S.; GUENTHER, F. neuralnet: Training of Neural Networks. 2016.

GALLAGHER, D. et al. Ankrd11 Is a Chromatin Regulator Involved in Autism that Is Essential for Neural Development. Developmental cell, 2014.

GAUGLER, T. et al. Most genetic risk for autism resides with common variation. Nature Genetics, v. 46, n. 8, p. 881-885, 20 jul. 2014.

GESCHWIND, D. H.; STATE, M. W. Gene hunting in autism spectrum disorder: on the path to precision medicine. The Lancet Neurology, v. 14, n. 11, p. 1109-1120, 1 nov. 2015.

GIRIRAJAN, S. et al. Refinement and discovery of new hotspots of copy-number variation associated with autism spectrum disorder. American journal of human genetics, v. 92, n. 2, p. 221-237, 7 fev. 2013.

GREENLAW, K. et al. A Bayesian group sparse multi-task regression model for imaging genetics. Bioinformatics, v. 33, n. 16, p. 2513-2522, 15 ago. 2017.

GRIESI-OLIVEIRA, K. et al. Modeling non-syndromic autism and the impact of TRPC6 disruption in human neurons. Molecular psychiatry, 11 nov. 2014.

GROVE, J. et al. Common risk variants identified in autism spectrum disorder. bioRxiv, p. 224774, 27 nov. 2017.

GUO, H. et al. Genetics of autism spectrum disorders. Zhong nan da xue xue bao. Yi xue ban $=$ Journal of Central South University. Medical sciences, v. 36, n. 8, p. 703711, ago. 2011. 
HAIR, J. F. et al. Análise multivariada de dados. Traducao. [s.l: s.n.].

HAMPTON, T. Early Brain Imaging in Infants May Help Predict Autism. JAMA, v. 318, n. 13, p. 1211-1212, 3 out. 2017.

HAND, D. J. Measuring classifier performance: a coherent alternative to the area under the ROC curve. Machine Learning, v. 77, n. 1, p. 103-123, 16 jun. 2009.

HARTIGAN, J. A.; WONG, M. A. Algorithm AS 136: A K-Means Clustering Algorithm. Applied Statistics, v. 28, n. 1, p. 100, 1979.

HASTIE, T.; TIBSHIRANI, R.; FRIEDMAN, J. The Elements of Statistical Learning. Traducao. New York, NY: Springer Science \& Business Media, 2013.

$\mathrm{HE}, \mathrm{X}$. et al. Integrated model of de novo and inherited genetic variants yields greater power to identify risk genes. PLoS Genetics, v. 9, n. 8, p. e1003671, 2013.

HOGART, A. et al. 15q11-13 GABAA receptor genes are normally biallelically expressed in brain yet are subject to epigenetic dysregulation in autism-spectrum disorders. Human Molecular Genetics, v. 16, n. 6, p. 691-703, 15 mar. 2007.

IOSSIFOV, I. et al. De novo gene disruptions in children on the autistic spectrum. Neuron, v. 74, n. 2, p. 285-299, 26 abr. 2012.

IOSSIFOV, I. et al. The contribution of de novo coding mutations to autism spectrum disorder. Nature, v. 515, n. 7526, p. 216-221, 29 out. 2014.

IOSSIFOV, I. et al. Low load for disruptive mutations in autism genes and their biased transmission. Proceedings of the National Academy of Sciences of the United States of America, v. 112, n. 41, p. E5600-E5607, 13 out. 2015.

JACQUEMONT, S. et al. A Higher Mutational Burden in Females Supports a "Female Protective Model" in Neurodevelopmental Disorders. American journal of human genetics, fev. 2014.

JIANG, Y.-H. et al. Detection of Clinically Relevant Genetic Variants in Autism Spectrum Disorder by Whole-Genome Sequencing. The American Journal of Human Genetics, jul. 2013.

KLEI, L. et al. Common genetic variants, acting additively, are a major source of risk for autism. Molecular autism, v. 3, n. 1, p. 9, 2012.

KOLEVZON, A. et al. A pilot controlled trial of insulin-like growth factor-1 in children with Phelan-McDermid syndrome. Molecular autism, v. 5, n. 1, p. 54, 2014.

KRISHNAN, A. et al. Genome-wide prediction and functional characterization of the genetic basis of autism spectrum disorder. Nature Neuroscience, 1 ago. 2016.

KRUMM, N. et al. Transmission Disequilibrium of Small CNVs in Simplex Autism. The American Journal of Human Genetics, set. 2013.

KRUMM, N. et al. Excess of rare, inherited truncating mutations in autism. Nature Genetics, v. 47, n. 6, p. 582-588, 11 maio 2015. 
KUMAR, R. A. Autism, mutations, and the environment: insights from exome sequencing. Clinical Genetics, v. 80, n. 4, p. 331-333, out. 2011.

LA TORRE-UBIETA, DE, L. et al. Advancing the understanding of autism disease mechanisms through genetics. Nature medicine, v. 22, n. 4, p. 345-361, abr. 2016.

LAPIN, V. et al. Regulating whole exome sequencing as a diagnostic test. Human Genetics, v. 135, n. 6, p. 655-673, jun. 2016.

LEK, M. et al. Analysis of protein-coding genetic variation in 60,706 humans. Nature, v. 536, n. 7616, p. 285-291, 17 ago. 2016.

LETAI, A. Functional precision cancer medicine-moving beyond pure genomics. Nature medicine, v. 23, n. 9, p. 1028-1035, 8 set. 2017.

LI, B. et al. Applying machine learning to identify autistic adults using imitation: An exploratory study. PLOS ONE, v. 12, n. 8, p. e0182652, 16 ago. 2017.

LI, H.; DURBIN, R. Fast and accurate short read alignment with Burrows-Wheeler transform. Bioinformatics, v. 25, n. 14, p. 1754-1760, 15 jul. 2009.

LI, J. et al. A comparative study of the genetic components of three subcategories of autism spectrum disorder. Molecular psychiatry, v. 383, p. 896, 6 jun. 2018.

LICHTENSTEIN, P. et al. The Genetics of Autism Spectrum Disorders and Related Neuropsychiatric Disorders in Childhood. American Journal of Psychiatry, v. 167, n. 11, p. 1357-1363, nov. 2010.

LIN, I.-F. et al. The effect of intranasal oxytocin versus placebo treatment on the autonomic responses to human sounds in autism: a single-blind, randomized, placebo-controlled, crossover design study. Molecular autism, v. 5, n. 1, p. 20, 28 fev. 2014.

LIU, L. et al. DAWN: a framework to identify autism genes and subnetworks using gene expression and genetics. Molecular autism, v. 5, n. 1, p. 22, 2014.

LORD, C.; BISHOP, S. L. Recent advances in autism research as reflected in DSM-5 criteria for autism spectrum disorder. Annual review of clinical psychology, v. 11, n. 1, p. 53-70, 2015.

LUDBROOK, J. Analysis of $2 \times 2$ tables of frequencies: matching test to experimental design. International journal of epidemiology, v. 37, n. 6, p. 1430-1435, dez. 2008.

LUO, W. et al. Systematic reconstruction of autism biology from massive genetic mutation profiles. Science Advances, v. 4, n. 4, p. e1701799, 1 abr. 2018.

LUO, X.; SOROCK, G. S. Analysis of recurrent event data under the case-crossover design with applications to elderly falls. Statistics in Medicine, v. 27, n. 15, p. 28902901, 2008.

MACHIELA, M. J. et al. Evaluation of polygenic risk scores for predicting breast and prostate cancer risk. Genetic epidemiology, v. 35, n. 6, p. 506-514, set. 2011. 
MANNING-COURTNEY, P. et al. Autism Spectrum Disorders. Current Problems in Pediatric and Adolescent Health Care, v. 43, n. 1, p. 2-11, jan. 2013.

MANRAI, A. K. et al. Genetic Misdiagnoses and the Potential for Health Disparities. New England Journal of Medicine, v. 375, n. 7, p. 655-665, 18 ago. 2016.

MARDIS, E. R. Next-generation DNA sequencing methods. Annu Rev Genomics Hum Genet, 2008.

MATTHIJS, G. et al. Guidelines for diagnostic next-generation sequencing. European Journal of Human Genetics, v. 24, n. 1, p. 2-5, 28 out. 2015.

MATULLO, G.; DI GAETANO, C.; GUARRERA, S. Next generation sequencing and rare genetic variants: From human population studies to medical genetics.

Environmental and molecular mutagenesis, p. 518-532, 6 ago. 2013.

MCKENNA, A. et al. The Genome Analysis Toolkit: a MapReduce framework for analyzing next-generation DNA sequencing data. Genome Research, v. 20, n. 9, p. 1297-1303, set. 2010.

MEYER, D. et al. e1071: Misc Functions of the Department of Statistics, Probability Theory Group (Formerly: E1071). 2017.

MICHAELSON, J. J. et al. Whole-Genome Sequencing in Autism Identifies Hot Spots for De Novo Germline Mutation. Cell, v. 151, n. 7, p. 1431-1442, dez. 2012.

MINGOTI, S. A. Análise de dados através de métodos de estatística multivariada: uma abordagem aplicada. Traducao. [s.l: s.n.].

MITCHELL, K. J. What is complex about complex disorders? Genome Biology, v. 13, n. 1, p. 237, 23 jan. 2012.

MOREIRA, E. S. et al. Detection of small copy number variations (CNVs) in autism spectrum disorder (ASD) by custom array comparative genomic hybridization (aCGH). Research in Autism Spectrum Disorders, v. 23, p. 145-151, mar. 2016.

MUGHAL, S. et al. Pheno4J: a gene to phenotype graph database. Bioinformatics, v. 33, n. 20, p. 3317-3319, 15 out. 2017.

NASLAVSKY, M. S. et al. Exomic variants of an elderly cohort of Brazilians in the ABraOM database. Human Mutation, v. 38, n. 7, p. 751-763, jul. 2017.

NEALE, B. M. et al. Patterns and rates of exonic de novo mutations in autism spectrum disorders. Nature News, v. 485, n. 7397, p. 242-245, 9 abr. 2013.

O'ROAK, B. J. et al. Exome sequencing in sporadic autism spectrum disorders identifies severe de novo mutations. Nature Genetics, v. 43, n. 6, p. 585-589, jun. 2011.

O'ROAK, B. J. et al. Sporadic autism exomes reveal a highly interconnected protein network of de novo mutations. Nature News, v. 485, n. 7397, p. 246-250, 10 maio 2012a. 
O'ROAK, B. J. et al. Multiplex targeted sequencing identifies recurrently mutated genes in autism spectrum disorders. Science, v. 338, n. 6114, p. 1619-1622, 21 dez. $2012 b$.

PEDERSEN, B. S.; QUINLAN, A. R. Who's Who? Detecting and Resolving Sample Anomalies in Human DNA Sequencing Studies with Peddy. The American Journal of Human Genetics, v. 100, n. 3, p. 406-413, mar. 2017.

PETROVSKI, S. et al. Genic Intolerance to Functional Variation and the Interpretation of Personal Genomes. PLoS Genetics, v. 9, n. 8, ago. 2013.

PINTO, D. et al. Convergence of Genes and Cellular Pathways Dysregulated in Autism Spectrum Disorders. The American Journal of Human Genetics, v. 94, n. 5, p. 677-694, 5 jan. 2014.

PIROOZNIA, M. et al. Validation and assessment of variant calling pipelines for next-generation sequencing. Human Genomics, v. 8, n. 1, p. 14, 30 jul. 2014.

QIN, J. A goodness-of-fit test for logistic regression models based on case-control data. Biometrika, v. 84, n. 3, p. 609-618, 1 set. 1997.

RAMACHANDRAN, S. et al. Support from the relationship of genetic and geographic distance in human populations for a serial founder effect originating in Africa. Proceedings of the National Academy of Sciences of the United States of America, v. 102, n. 44, p. 15942-15947, 1 nov. 2005.

RETTERER, K. et al. Clinical application of whole-exome sequencing across clinical indications. Genetics in Medicine, v. 18, n. 7, p. 696-704, jul. 2016.

REVELLE, W. psych: Procedures for Personality and Psychological Research, Northwestern University, Evanston, Illinois, USA. 2017.

RICHARDS, S. et al. Standards and guidelines for the interpretation of sequence variants: a joint consensus recommendation of the American College of Medical Genetics and Genomics and the Association for Molecular Pathology. Genetics in Medicine, 5 mar. 2015.

RIDLEY, M. Evolution. Traducao. [s.1.] Wiley-Blackwell, 2009.

RIEDMILLER, M.; (null), H. B.; 1993. A direct adaptive method for faster backpropagation learning: The RPROP algorithm. Neural Networks, IEEE International Conference on, 1993.

RIIKONEN, R. Treatment of autistic spectrum disorder with insulin-like growth factors. European Journal of Paediatric Neurology, ago. 2016.

RONEMUS, M. et al. The role of de novo mutations in the genetics of autism spectrum disorders. Nature Publishing Group, v. 15, n. 2, p. 133-141, fev. 2014.

ROTSCHAFER, S. E.; MARSHAK, S.; CRAMER, K. S. Deletion of Fmr1 Alters Function and Synaptic Inputs in the Auditory Brainstem. PLoS ONE, v. 10, n. 2, p. e0117266, 13 fev. 2015. 
SAMOCHA, K. E. et al. A framework for the interpretation of de novo mutation in human disease. Nature Genetics, v. 46, n. 9, p. 944-, set. 2014.

SANDERS, S. J. et al. De novo mutations revealed by whole-exome sequencing are strongly associated with autism. Nature, v. 485, n. 7397, p. 237-241, 10 maio 2012.

SANDIN, S. et al. The familial risk of autism. JAMA, v. 311, n. 17, p. 1770-1777, 7 maio 2014.

SANDIN, S. et al. The Heritability of Autism Spectrum Disorder. JAMA, v. 318, n. 12, p. 1182-1184, 26 set. 2017.

SCHAEFER, G. B.; MENDELSOHN, N. J. Clinical genetics evaluation in identifying the etiology of autism spectrum disorders: 2013 guideline revisions. Genetics in Medicine, v. 15, n. 5, p. 399-407, 21 mar. 2013.

SCHERER, S. W.; DAWSON, G. Risk factors for autism: translating genomic discoveries into diagnostics. Human Genetics, v. 130, n. 1, p. 123-148, jul. 2011.

SCHIZOPHRENIA WORKING GROUP OF THE PSYCHIATRIC GENOMICS CONSORTIUM. Biological insights from 108 schizophrenia-associated genetic loci. Nature News, v. 511, n. 7510, p. 421-427, 24 jul. 2014.

SCHORK, N. J. Genetics of complex disease: approaches, problems, and solutions. American Journal of Respiratory and Critical Care Medicine, v. 156, n. 4 Pt 2, p. S103-9, out. 1997.

SEBAT, J. et al. Strong association of de novo copy number mutations with autism. Science, v. 316, n. 5823, p. 445-449, 20 abr. 2007.

SHEMESH, E. et al. Effect of Intranasal Insulin on Cognitive Function: A Systematic Review. The Journal of clinical endocrinology and metabolism, v. 97, n. 2, p. 366376, fev. 2012.

SOBREIRA, N. et al. New tools for Mendelian disease gene identification: PhenoDB variant analysis module; and GeneMatcher, a web-based tool for linking investigators with an interest in the same gene. Human Mutation, v. 36, n. 4, p. 425431, abr. 2015.

STEIN, J. L.; PARIKSHAK, N. N.; GESCHWIND, D. H. Rare inherited variation in autism: beginning to see the forest and a few trees. Neuron, v. 77, n. 2, p. 209-211, 23 jan. 2013.

STEINBERG, J.; WEBBER, C. The roles of FMRP-regulated genes in autism spectrum disorder: single- and multiple-hit genetic etiologies. American journal of human genetics, v. 93, n. 5, p. 825-839, 7 nov. 2013.

STESSMAN, H. A. F. et al. Targeted sequencing identifies 91 neurodevelopmentaldisorder risk genes with autism and developmental-disability biases. Nature

Genetics, v. 49, n. 4, p. 515-526, 1 abr. 2017.

SWETS, J. A.; DAWES, R. M.; MONAHAN, J. Better decisions through science.

Scientific American, v. 283, n. 4, p. 82-87, out. 2000. 
SZATMARI, P. et al. Mapping autism risk loci using genetic linkage and chromosomal rearrangements. Nature Genetics, v. 39, n. 3, p. 319-328, 18 fev. 2007.

TEAM, R. C. R: A language and environment for statistical computing. R Foundation for Statistical Computing, Vienna, Austria. 1 jan. 2017.

THERNEAU, T.; ATKINSON, B.; RIPLEY, B. rpart: Recursive Partitioning and Regression Trees. 2017.

TORDJMAN, S. et al. Gene $\times$ Environment Interactions in Autism Spectrum Disorders: Role of Epigenetic Mechanisms. Schizophrenia, v. 5, 4 ago. 2014.

TRUJILLANO, D. et al. Clinical exome sequencing: results from 2819 samples reflecting 1000 families. European Journal of Human Genetics, 16 nov. 2016.

TURNER, T. N. et al. Genomic Patterns of De Novo Mutation in Simplex Autism. Cell, v. 171, n. 3, p. 710-722.e12, 19 out. 2017.

UDDIN, M. et al. Brain-expressed exons under purifying selection are enriched for de novo mutations in autism spectrum disorder. Nature Genetics, 25 maio 2014.

VAISHNAVI, V. et al. Mining the 3'UTR of Autism-implicated Genes for SNPs Perturbing MicroRNA Regulation. Genomics, Proteomics \& Bioinformatics, abr. 2014.

WANG, J. et al. Genome measures used for quality control are dependent on gene function and ancestry. Bioinformatics, v. 31, n. 3, p. 318-323, 2015.

WANG, K.; LI, M.; HAKONARSON, H. ANNOVAR: functional annotation of genetic variants from high-throughput sequencing data. Nucleic Acids Research, v. 38, n. 16, p. e164-e164, set. 2010.

WEI, T.; SIMKO, V. R package "corrplot": Visualization of a Correlation Matrix (Version 0.84). 2017.

WEINER, D. J. et al. Polygenic transmission disequilibrium confirms that common and rare variation act additively to create risk for autism spectrum disorders. Nature Genetics, v. 450, p. 86-11, 15 maio 2017.

WEISS, L. A. et al. A genome-wide linkage and association scan reveals novel loci for autism. Nature News, v. 461, n. 7265, p. 802-808, 8 out. 2009.

WERLING, D. M. et al. Limited contribution of rare, noncoding variation to autism spectrum disorder from sequencing of 2,076 genomes in quartet families. bioRxiv, p. 127043, 13 abr. 2017.

WICKHAM, H. ggplot2: Elegant Graphics for Data Analysis. Springer-Verlag New York, 2009.

WOODBURY-SMITH, M. et al. Using extended pedigrees to identify novel autism spectrum disorder (ASD) candidate genes. Human Genetics, v. 134, n. 2, p. 191-201, 2015. 
WOODBURY-SMITH, M.; SCHERER, S. W. Progress in the genetics of autism spectrum disorder. Developmental Medicine \& Child Neurology, v. 60, n. 5, p. 445-451, maio 2018.

WU, J.; LI, Y.; JIANG, R. Integrating Multiple Genomic Data to Predict DiseaseCausing Nonsynonymous Single Nucleotide Variants in Exome Sequencing Studies. PLoS Genetics, v. 10, n. 3, mar. 2014.

WU, X. et al. Top 10 algorithms in data mining. Knowledge and Information Systems, v. 14, n. 1, p. 1-37, 4 dez. 2007.

XUE, Y. et al. Deleterious-and Disease-Allele Prevalence in Healthy Individuals: Insights from Current Predictions, Mutation Databases, and Population-Scale Resequencing. American journal of human genetics, v. 91, n. 6, p. 1022-1032, 7 dez. 2012.

YAMAMOTO, G. L. et al. Mutations in PCYT1A cause spondylometaphyseal dysplasia with cone-rod dystrophy. American journal of human genetics, v. 94, n. 1, p. 113-119, 2 jan. 2014.

YU, T. W. et al. Using Whole-Exome Sequencing to Identify Inherited Causes of Autism. Neuron, v. 77, n. 2, p. 259-273, jan. 2013.

YUEN, R. K. C. et al. Whole-genome sequencing of quartet families with autism spectrum disorder. Nature medicine, 26 jan. 2015.

YUEN, R. K. et al. Genome-wide characteristics of de novo mutations in autism. npj Genomic Medicine, v. 1, p. 16027, 3 ago. 2016.

ZARE, M.; REZVANI, Z.; BENASICH, A. A. Automatic classification of 6-month-old infants at familial risk for language-based learning disorder using a support vector machine. Clinical Neurophysiology, v. 127, n. 7, p. 2695-2703, jul. 2016.

ZHAO, Y.; PAN, Q.; DU, C. Logistic Regression Augmented Community Detection for Network Data with Application in Identifying Autism-Related Gene Pathways. Biometrics, 24 jul. 2018.

ZHENG, X. et al. A high-performance computing toolset for relatedness and principal component analysis of SNP data. Bioinformatics, v. 28, n. 24, p. 3326-3328, 1 dez. 2012. 


\section{Attachments}

\subsection{Attachment 1}

AAK1 ANP32E ATRNL1 C3orf17 CDC5L CNOT2 DEPDC5 EIF4G3 ABCA2 AP1G1 ATXN1 C4orf21 CDH10 CNOT6 DGCR8 EIF6 ABCA3 AP1S1 ATXN2 C5orf42 CDH18 CNTN1 DGKB ELAVL1 ABCC5 AP3B1 ATXN3 C5orf45 CDH20 CNTN2 DGKD ELAVL3 ABCC8 AP3B2 ATXN7L3B C8orf34 CDH22 CNTN4 DGKG ELAVL4 ABCD2 AP3M2 AUTS2 C8orf46 CDH23 CNTN6 DGKH ELK4 ABCG2 AP4B1 AVIL C9orf72 CDH24 CNTNAP1 DGKI ELMO1 ABCG4 AP5M1 B3GAT1 CA10 CDH7 CNTNAP2 DHX36 ELMO2 ABI2 APBA1 B4GALNT1 CA11 CDHR3 CNTNAP4 DICER1 ELMOD1 ACACA APBA2 B4GALNT4 CA8 CDIP1 CNTNAP5 DIRAS2 ELOVL4 ACAP3 APBB1 B4GALT6 CABP1 CDK13 COA5 DISP2 ELP4 ACBD3 APBB2 BAALC CACHD1 CDK14 COL13A1 DIXDC1 EMC4 ACSL3 APC BAI1 CACNA1A CDK17 COL19A1 DLG1 EML5 ACSL6 APLP1 BAI2 CACNA1B CDK5R1 COL27A1 DLG2 ENAH ACTL6B APLP2 BAI3 CACNA1D CDK8 CORO2B DLG3 ENO2 ADAM11 APOO BARHL2 CACNA1E CDKL5 CPD DLG4 ENOX1 ADAM22 APP BAZ2B CACNA1G CDON CPLX1 DLGAP1 ENY2 ADAM23 APPL1 BBS7 CACNA2D1 CDR2L CPLX2 DLGAP4 EOMES ADAMTS16 AQP4 BCAN CACNA2D2 CDS1 CPLX3 DLK2 EP400 ADAMTS18 AQR BCAS1 CACNB3 CDYL2 CPNE6 DMTF1 EPB41 ADARB1 ARGLU1 BCAS3 CACNB4 CECR2 CPNE9 DMXL1 EPB41L1 ADCY1 ARHGAP19-SLIT1 BCL11A CACNG2 CELF3 CPT1C DMXL2 EPB41L3

ADCY8 ARHGAP22 BCL2L2 CACNG7 CELF4 CRAMP1L DNAJB2 EPB41L4B

ADCYAP1R1 ARHGAP26 BCL2L2-PABPN1 CADM1 CELF5 CRB1 DNAJC17 EPB41L5

ADD1 ARHGAP29 BDP1 CADM3 CELF6 CRHR1 DNAJC6 EPC2 ADD2 ARHGAP33 BEGAIN CADM4 CELSR2 CRMP1 DNAJC7 EPHA6 ADD3 ARHGAP44 BEND6 CADPS CELSR3 CRTAM DNER EPHA7 AEBP2 ARHGDIG BIN1 CADPS2 CENPT CRTC1 DNM1 EPHB1 AES ARHGEF11 BIVM CALB2 CEP120 CRY1 DNMT1 EPHB2 AFF2 ARHGEF25 BIVM-ERCC5 CALN1 CEP170 CSMD1 DNMT3A ERC1 AFG3L1P ARHGEF4 BLCAP CAMK2B CEP192 CSPG5 DOCK3 ERC2 AFTPH ARHGEF7 BMPER CAMK2G CEP95 CSRNP3 DOCK4 ERCC3 AGAP1 ARID4A BMPR2 CAMK4 CERCAM CSTF3 DOCK9 ERCC6 AGAP2 ARID4B BPTF CAMKK1 CERKL CTIF DOK6 ERGIC2 AGAP3 ARL1 BRD8 CAMKK2 CERS5 CTNNB1 DOPEY1 ERH 
AGPAT4 ARL8A BRD9 CAMKV CERS6 CTNND2 DOPEY2 ERP44 AGTPBP1 ARMC8 BRSK1 CAMSAP1 CFL1 CTPS2 DPF1 ESYT3 AHI1 ARMC9 BRSK2 CAMSAP2 CHD2 CTTNBP2 DPF3 ETV1 AJAP1 ARMCX5 BRWD1 CAMTA1 CHD3 CUL9 DPP10 EVL AKAP10 ARMCX5-GPRASP2 BRWD3 CAND1 CHD6 CWC27 DPP6 EXOC1

AKAP11 ARNT2 BSDC1 CAPRIN2 CHD9 CWF19L2 DPYSL2 EXOC2 AKAP9 ARPP21 BSN CASD1 CHL1 CXorf23 DPYSL3 EXOSC10 AKD1 ARVCF BTBD1 CASK CHM CXXC1 DPYSL5 EXT2 AKT3 ASAP1 BTBD10 CASKIN1 CHN1 CYP26B1 DRG1 EZH1 ALS2 ASB3 BTBD9 CATSPER2 CHRD CYP46A1 DROSHA FADS2 AMER2 ASIC1 BTN2A2 CBLN1 CHRNB2 DAAM1 DTNA FAIM2 AMIGO1 ASIC2 BTRC CBX1 CHRNB3 DAB1 DTNB FAM120A AMPH ASPHD1 C10orf35 CCAR1 CHST10 DAB2IP DTX3 FAM120C AMZ2 ASPHD2 C11orf30 CCBL1 CIC DACT1 DUSP11 FAM126B ANAPC5 ASTN1 C12orf29 CCDC111 CIT DAGLA DVL2 FAM131B ANGEL2 ASTN2 C12orf76 CCDC115 CKAP5 DBC1 DYNC1H1 FAM135A ANK1 ASXL1 C14orf132 CCDC132 CLASP1 DCAF17 DYNC1LI2 FAM13B ANK2 ATCAY C14orf2 CCDC136 CLASP2 DCAF8 DYRK1A FAM160A2 ANK3 ATF2 C14orf37 CCDC6 CLCN3 DCC DZIP3 FAM168A ANKRD12 ATG4C C15orf27 CCDC88A CLCN6 DCLK1 E4F1 FAM171A2 ANKRD13B ATL1 C17orf85 CCDC92 CLIP2 DCLK2 EBAG9 FAM171B ANKRD13C ATP1B2 C18orf32 CCNC CLIP3 DCTN1 ECE2 FAM179B ANKRD17 ATP2B1 C1orf126 CCNG2 CLK4 DCTN2 EDIL3 FAM188A ANKRD26 ATP2B2 C1orf51 CCNJL CLSTN3 DCUN1D4 EFHA2 FAM193A ANKRD6 ATP2B3 C1orf61 CCNT2 CLTC DDX26B EFR3B FAM199X ANKS1B ATP2C1 C1QTNF3 CCNY CLUHP3 DDX42 EHBP1 FAM19A5 ANKS6 ATP6V1G2 C1QTNF3-AMACR CCP110 CLVS1 DDX6 EHMT1 FAM216A

ANKZF1 ATP6V1G2-DDX39B C20orf112 CCSAP CMC2 DEAF1 EIF2B5 FAM217B

ANLN ATP8A1 C20orf24 CD200 CNDP1 DEF8 EIF4A2 FAM228B ANO10 ATP8A2 C20orf27 CDC42BPA CNIH2 DENND4A EIF4E3 FAM5B ANO4 ATRN C2CD5 CDC42BPB CNKSR2 DENND5B EIF4G2 FAM5C FAM73B GAP43 HAUS3 KCNAB3 KRT222 MAP4K3 MTERFD2 NKAIN3 FAM98B GARNL3 HCN1 KCNC3 KSR2 MAP4K4 MTHFD1L NKIRAS1 FAN1 GDAP1L1 HDAC2 KCND2 L1CAM MAP4K5 MTMR9 NKTR FANCL GDPD1 HECTD2 KCND3 LANCL1 MAP6D1 MTPAP NLGN1 FAT2 GEMIN7 HECTD4 KCNH1 LAS1L MAP7D2 MTSS1L NLGN2 FAXC GFAP HECW1 KCNH7 LATS1 MAPK1 MTUS1 NLGN3 FBRSL1 GFOD1 HERC1 KCNIP1 LCOR MAPK10 MUM1 NLGN4X FBXL14 GHITM HERC3 KCNIP4 LDLRAD3 MAPK4 MYCBP2 NLK FBXL16 GIGYF2 HERPUD2 KCNJ6 LEMD3 MAPK8 MYH10 NMNAT2 FBXL20 GIT2 HINFP KCNK1 LENG8 MAPK8IP1 MYO15A NNAT 
FBXL3 GLCCI1 HIPK1 KCNK10 LENG9 MAPK8IP3 MYO5A NOL4 FBXO11 GLCE HIPK2 KCNK12 LEO1 MAPK9 MYT1 NOP58 FBXO16 GLMN HIST1H2BC KCNK9 LGALSL MAPRE3 MYT1L NOVA1 FBXO27 GLRA2 HIST1H4E KCNMB4 LGI1 MAPT N4BP2L2 NOVA2 FBXO3 GLRB HIVEP2 KCNN1 LGI3 MARCH8 NAA38 NPAS3 FBXO9 GLT25D2 HMG20A KCNN2 LHFPL4 MARK1 NAB1 NPAT FBXW11 GMFB HMGCLL1 KCNQ2 LIMK1 MARK4 NAE1 NPFFR1 FBXW7 GNAI1 HMGCR KCNQ3 LIN7C MAST1 NALCN NPM2 FER GNAL HMP19 KCTD13 LIX1 MBD5 NAP1L2 NPPA-AS1 FEZ1 GNAO1 HNRNPA0 KCTD3 LLGL1 MBOAT2 NAP1L5 NPR2 FGF14 GNAZ HNRNPH3 KCTD6 LMAN1L MBTPS2 NAPB NPTN FGF17 GNB3 HNRNPR KCTD8 LMBR1 MCF2L NAV2 NPTX1 FGF5 GNB5 HOOK3 KDM2B LMBRD1 MCF2L2 NAV3 NR1D1 FGFR2 GNL1 HP1BP3 KDM4C LMNB1 MCTP1 NBAS NRBP1 FIG4 GNPDA2 HPCA KHDRBS2 LMTK2 MCU NBEA NRCAM FLT3 GNPTAB HPCAL4 KHDRBS3 LOC100131825 MDGA1 NCAM1 NRD1

FMN2 GOLM1 HR KIAA0319 LOC100379224 MDN1 NCAM2 NRIP2 FMNL2 GPM6A HRH3 KIAA0408 LONRF2 MEA1 NCAN NRSN1 FMR1 GPM6B HS3ST1 KIAA0430 LPHN1 MED12L NCBP1 NRSN2 FN1 GPR107 HSF4 KIAA0513 LPHN3 MED13L NCDN NRXN2 FNBP4 GPR158 ICA1L KIAA0895L LPPR2 MEGF10 NCKAP1 NRXN3 FOCAD GPR158-AS1 IDUA KIAA0930 LRCH1 MEGF11 NCOA1 NSD1 FOXJ3 GPR180 IFT46 KIAA1033 LRCH2 MEGF8 NCOA2 NSF FOXK2 GPR37L1 IGFBP7 KIAA1109 LRCH3 METTL10 NCOA6 NSG1 FOXN2 GPR52 IGSF21 KIAA1191 LRP1B METTL21A NCOA7 NTM FOXP1 GPR75-ASB3 IGSF8 KIAA1199 LRRC40 METTL21D NCOR1 NTRK2

FRMD5 GPR83 IKBKAP KIAA1324L LRRC7 MGARP NCS1 NTRK3 FRS2 GPR98 IL16 KIAA1456 LRRFIP2 MGAT3 NDFIP1 NUAK1 FRY GPRASP1 INPP4A KIAA1468 LRRTM3 MGAT4A NDP NUDT3 FSD1 GPRASP2 INPP5F KIAA1598 LRRTM4 MGAT5B NDRG2 NUFIP1 FSTL4 GRAMD1B INTS3 KIAA1704 LSAMP MID1 NDRG3 NUFIP2 FUBP3 GRIA1 INTS4 KIAA1841 LSS MINK1 NDRG4 NUP133 FUT8 GRIA2 INTU KIF1A LTN1 MIR497HG NDST3 NUP153 FUT9 GRIA3 IRX2 KIF1B LUC7L MLL NDUFA6 NUP93 FXYD7 GRIA4 ITFG1 KIF21A LY6G5B MLL2 NEDD4L NXPH3 FZD3 GRID1 ITM2C KIF2A LY6H MLL3 NEFH OCIAD1 FZR1 GRID2 ITPR1 KIF3A LYPLAL1 MLL5 NEFL OGFRL1 G3BP2 GRIK1 ITSN1 KIF3C LYST MLST8 NEFM OLFM1 GABBR1 GRIK2 IWS1 KIF5A MACF1 MMP16 NEGR1 OLFM2 GABBR2 GRIK5 JAK2 KIF5B MACROD2 MMP24 NEK1 OLFM3 GABRA1 GRIN1 JAKMIP2 KIF5C MADD MMS19 NELL2 OPCML GABRA2 GRIN2A JAM2 KIFAP3 MAG MOG NEMF OPHN1 
GABRA3 GRIN2B JARID2 KIFC2 MAGEE1 MON2 NEO1 OSBP GABRA6 GRIN2C JMJD1C KIN MAGI1 MORN1 NETO2 OSBP2 GABRB1 GRM1 JMJD7 KLC2 MAGI2 MPDZ NEURL4 OSBPL2 GABRB2 GRM3 JMJD7-PLA2G4B KLHL18 MAGI3 MPHOSPH6 NEUROD2 OSBPL6

GABRB3 GRM4 JMY KLHL2 MAGOHB MPHOSPH9 NEUROD6 OSBPL7 GABRD GRM5 JPH3 KLHL24 MAML3 MPP6 NF1 OSBPL8 GABRG1 GSTA4 JPH4 KLHL3 MANEAL MREG NF2 OTUB1 GABRG2 GTF2H4 KALRN KLHL32 MAP1A MRPL50 NFASC OTUD6B GAD1 GTPBP10 KANSL2 KLHL5 MAP2 MRPS21 NFAT5 OTUD7A GAD2 GUSBP4 KAT2A KLHL8 MAP2K5 MRPS30 NINL P2RX7 GALNT13 HABP4 KAT6B KNDC1 MAP3K10 MSANTD3-TMEFF1 NIPA1 P4HTM

GALNT5 HACE1 KBTBD3 KPNA3 MAP3K12 MSANTD4 NIPAL3 PAAF1 GALNT9 HAPLN2 KCNA2 KREMEN2 MAP4 MSH2 NKAIN1 PACS1 GAN HAUS2 KCNAB1 KRIT1 MAP4K2 MTA3 NKAIN2 PACSIN1 PAGR1 PLD5 PSIP1 RFC1 SEC31B SMAD4 STX1B TMC2 PAK1 PLEKHA6 PSME3 RFTN2 SEC62 SMARCA2 STX6 TMCC2 PAK3 PLEKHB1 PSPC1 RFWD2 SECISBP2L SMARCAD1 STXBP1 TMEFF1 PAK7 PLEKHG5 PTCHD1 RFX3 SEL1L3 SMARCD3 STXBP5 TMEFF2 PALM PLK2 PTK2 RGS11 SEMA4F SMC1A STXBP5-AS1 TMEM116 PANK4 PLXDC2 PTK2B RGS12 SEMA6A SMG1 SULT4A1 TMEM130 PAPD5 PLXNA3 PTN RGS7 SENP6 SMG7 SUPT3H TMEM132A PAPL PLXNB3 PTPN13 RGS8 SENP7 SNAP25 SUPT7L TMEM135 PAPSS1 PMP2 PTPN5 RHBDL1 SEP15 SNAPC3 SUV420H1 TMEM14C PAQR6 PMPCB PTPRA RHOT1 SEPT3 SNCA SUV420H2 TMEM170A PARD6A PMVK PTPRD RICTOR SEPT4 SNCAIP SV2A TMEM175 PARP2 POGZ PTPRG-AS1 RIF1 SEPT8 SNCB SV2B TMEM180 PAX6 POLA1 PTPRN RIMS1 SERF2 SNTG1 SYBU TMEM189-UBE2V1 PAXIP1 POLB PTPRR RIMS2 SERF2-C15ORF63 SNX14 SYN1 TMEM196 PCBP3 POLE PTPRS RIMS3 SERGEF SNX2 SYN2 TMEM200B PCCA POLR3A PTPRZ1 RIT2 SEZ6 SNX25 SYN3 TMEM241 PCDH9 POLR3F PUM2 RLF SEZ6L SNX27 SYNDIG1 TMEM25 PCDHGC3 POU2F1 PVALB RNF112 SEZ6L2 SOGA1 SYNE1 TMEM30A PCF11 PPARGC1B PYURF RNF145 SF3B1 SOGA2 SYNGR1 TMEM50B PCGF3 PPFIA1 QSER1 RNF146 SFPQ SOGA3 SYNJ1 TMEM59L PCIF1 PPFIA2 QSOX2 RNF165 SGIP1 SORBS2 SYNPR TMEM74 PCLO PPFIA3 R3HDM1 RNF182 SGK196 SORL1 SYP TMEM9B PCNXL2 PPID R3HDM2 RNFT2 SGTB SORT1 SYT1 TMTC2 PCYOX1L PPIP5K1 RAB11FIP4 RNGTT SH3D21 SOS1 SYT11 TNIK PDE1A PPME1 RAB15 RPAIN SH3GL2 SOS2 SYT12 TNK2 PDE3B PPP1R11 RAB1A RPAP3 SH3GLB2 SOX2 SYT13 TNKS2 PDE4B PPP1R12A RAB26 RPH3A SHANK1 SPAG9 SYT14 TNPO2 PDIA2 PPP1R16B RAB37 RPL17-C18orf32 SHANK2 SPAST SYT2 TNR 
PDP1 PPP1R9B RAB39B RPRD1A SHC3 SPIN3 SYT3 TNRC6A PDS5A PPP2CA RAB3A RPRD2 SHF SPNS1 SYT4 TNRC6C PDS5B PPP2R2B RAB3C RPS10-NUDT3 SHISA6 SPOCK2 SYT5 TOP2B PDXP PPP2R2C RAB3GAP2 RPS6KA5 SHPRH SPOCK3 SYT9 TOP3A PDZD4 PPP2R2D RAB5A RPS6KL1 SIPA1L1 SPRYD3 TAB2 TP53BP1 PEA15 PPP2R5B RAB6B RSBN1 SLAIN1 SPTAN1 TAF15 TPCN1 PEG10 PPP3CA RABGAP1L RTEL1 SLBP SPTBN2 TAF1C TPD52L2 PEG3 PPP3R1 RALGAPA1 RTEL1-TNFRSF6B SLC12A2 SPTBN4 TAF2 TPGS2

PELI3 PPP6R2 RALGAPB RTF1 SLC12A5 SRCIN1 TAGLN3 TRA2A PER3 PRDM10 RALGDS RTN1 SLC17A7 SRGAP3 TAOK3 TRAK2 PEX1 PRDM15 RALGPS1 RUFY2 SLC1A6 SRI TARBP1 TRAPPC10 PGAP1 PRDM2 RALYL RUFY3 SLC22A15 SRRM4 TBC1D19 TRAPPC11 PGBD5 PRDX3 RAP1GAP2 RUNDC3A SLC22A17 SRSF11 TBC1D5 TRAPPC12

PGM2L1 PREP RAP1GDS1 RUNDC3B SLC23A2 SRSF7 TBC1D9B TRAPPC8

PHACTR3 PREX2 RAP2A RUNX1T1 SLC24A2 SRSF9 TBCEL TRIM23 PHF14 PRKACB RAPGEF2 RUSC1 SLC24A3 SS18L1 TBCK TRIM3 PHF21A PRKAG2 RAPGEF4 RYBP SLC25A27 SSTR2 TBRG1 TRIM33 PHKB PRKAR2B RAPGEF6 SALL2 SLC25A40 ST18 TCEAL2 TRIM46 PHTF1 PRKCE RASGEF1A SAMD14 SLC26A10 ST6GAL2 TCEB2 TRIM67 PHYHIP PRKCG RASGEF1C SAR1A SLC26A5 ST8SIA1 TCF12 TRIM8 PHYHIPL PRMT7 RASGRF1 SART3 SLC30A9 ST8SIA3 TCF4 TRIM9 PI4KA PRMT8 RASGRP1 SATB2 SLC32A1 ST8SIA5 TCTN1 TRIO PIGY PROSAPIP1 RB1 SBF2 SLC36A4 STAM2 TDRKH TRIP12 PIGZ PROSER1 RBFOX1 SCAI SLC4A10 STAMBPL1 TEF TRNAU1AP PIK3C3 PRPF18 RBM15 SCAPER SLC4A7 STARD4 TELO2 TRO PIK3R2 PRPF31 RBM39 SCG3 SLC4A8 STAU1 TENM1 TRPC1 PIP4K2B PRPF38B RCAN2 SCN1A SLC6A1 STK19 TFDP1 TRPC3 PIP5K1C PRPF39 RCBTB1 SCN2A SLC6A15 STK32C TGIF2-C20orf24 TRPC4AP

PITPNA PRPF6 RCOR3 SCN2B SLC6A7 STK39 THAP10 TRPM2 PITPNC1 PRR3 RDH13 SCN3A SLC7A14 STMN1 THOC7 TRPM3 PITPNM1 PRRC2C REC8 SCN3B SLC9A5 STMN2 THRA TRRAP PITPNM3 PRRT1 REEP2 SCN4B SLC9A6 STMN3 THUMPD2 TSC1 PKD1 PRRT2 REEP5 SCN8A SLCO1A2 STMN4 TIAM1 TSEN2 PLA2G4B PRTFDC1 RELL2 SCRN3 SLIT1 STRC TIAM2 TSPAN5 PLCB1 PRUNE2 RELN SDC3 SLITRK3 STRIP1 TLE2 TSPYL4 PLCB4 PSD REPS1 SDCCAG8 SLITRK5 STRN4 TLK1 TTC21B PLCG1 PSD2 REV1 SEC24B SLK STX16 TLL1 TTC33 PLCL2 PSEN1 REV3L SEC31A SLX4 STX1A TM2D3 TTC37 TTC39B ULK2 VAMP2 WDR27 YTHDF1 ZFYVE27 ZNF25 ZNF532 TTLL11 UNC5C VAPB WDR41 YTHDF2 ZFYVE28 ZNF250 ZNF536 
TTLL7 UNC79 VAT1L WDR47 YY1 ZFYVE9 ZNF256 ZNF540 TUB UNC80 VOPP1 WDR67 ZBTB41 ZIC1 ZNF280D ZNF566 TUBB2A UPF3B VPS13C WDR7 ZC2HC1A ZIC2 ZNF287 ZNF568 TUBB2B URI1 VPS18 WDSUB1 ZC3H15 ZIC3 ZNF335 ZNF585A TUBB3 USP16 VPS29 WHSC1L1 ZCCHC12 ZIC4 ZNF343 ZNF589 TUBGCP6 USP20 VPS33B WRAP73 ZDHHC17 ZIM2 ZNF362 ZNF605 TULP4 USP21 VPS37A WSCD1 ZDHHC21 ZMYM2 ZNF365 ZNF702P TUSC3 USP31 VPS41 WSCD2 ZDHHC4 ZMYM4 ZNF382 ZNF721 TYRO3 USP32 VPS53 WWC3 ZDHHC8 ZMYND11 ZNF385D ZNF738 UBAP2L USP33 VPS54 XKR6 ZEB1 ZMYND8 ZNF418 ZNF740 UBE2K USP34 VSX1 XPO7 ZFAND1 ZNF133 ZNF461 ZNF75A UBE2O USP45 VWC2 XRCC1 ZFC3H1 ZNF136 ZNF471 ZNF764 UBE2V1 USP46 WASF1 XYLT1 ZFHX2 ZNF138 ZNF483 ZNF767 UBP1 USP48 WASF3 YEATS2 ZFP1 ZNF175 ZNF490 ZNF778 UBQLN1 USP9Y WASL YLPM1 ZFP112 ZNF211 ZNF493 ZNF793 UBTD2 UTP18 WDFY3 YME1L1 ZFP30 ZNF224 ZNF521 ZNF85 UBTF UTP23 WDR17 YPEL4 ZFPM2 ZNF236 ZNF528 ZP2 UCK1 UXS1 WDR19 YTHDC2 ZFYVE16 ZNF238 ZNF529 ZSWIM8

\subsection{Attachment 2}

AKT1 BTRC HDAC1 PYGO2 SOX2 SOX9 TLE1 UBB AKT2 CBY1 KMT2D RBBP5 SOX3 SRY TLE2 UBC APC CHD8 LEF1 RPS27A SOX4 TCF7 TLE3 XIAP ASH2L CTBP1 MEN1 SOX13 SOX6 TCF7L1 TLE4 XPO1 BCL9 CTNNB1 PYGO1 SOX17 SOX7 TCF7L2 UBA52 YWHAZ BCL9L CTNNBIP1

\subsection{Attachment 3}

5S_rRNA AMPD1 BCAS1 CD2BP2 CTNNB1 DNMT1 DRD1 FER 7SK AMT BCKDK CD38 CTTNBP2 DNMT3A DRD2 FEZF2 AANAT ANK2 BCL11A CD44 CUBN DNMT3B DRD3 FGA AB209061 ANK3 BCL7B CD99 CUL2 DOC2A DRP2 FGD1 ABAT ANKRD11 BCL7C CD99L2 CUL3 DOCK10 DST FGFBP3 ABCA1 ANXA1 BCL9 CDC42BPB CUL4B DOCK11 DTNA FHIT ABCA7 ANXA5 BDNF CDC45 CX3CR1 DOCK4 DUSP22 FKBP6 ABCC1 ANXA7 BDNF-AS CDH10 CXCR3 DOLK DYDC1 FLJ00278 ABCC6 AP1S2 BGN CDH11 CXorf36 DPP10 DYDC2 FLJ00285 ABHD11 APBA2 BIN1 CDH22 CYBB DPP3 DYRK1A FLJ00322 
ABI2 APC BIRC6 CDH8 CYFIP1 DPP6 EEF1A2 FLNA

ABL1 APH1A BOLA2 CDH9 CYP11B1 DPYD EFR3A FLT1

abParts APOBR BRAF CDIPT CYP19A1 DQ571461 EGR2 FMO5

ACP6 APP BRCA2 CDIPT-AS1 DAB1 DQ572979 EHMT1 FMR1

ACR AQP4 BRSK2 CDK2 DAGLA DQ574263 EIF1AY FOLH1

ACTN4 AR BRWD1 CDK4 DAPK1 DQ575242 EIF2S3 FOPNL

ACY1 ARHGAP11B BRWD3 CDKL5 DAPP1 DQ575284 EIF3C FOXG1

ADA ARHGAP24 BX648073 CDKN1B DAZ1 DQ575576 EIF3CL FOXP1

ADAMTS18 ARHGAP32 BZRAP1 CECR2 DAZ2 DQ575741 EIF4E FOXP2

ADARB1 ARHGDIA C11orf30 CELF4 DAZ3 DQ576952 EIF4EBP2 FRK

ADCY5 ARHGEF9 C12orf57 CELF6 DAZ4 DQ577333 EIF4H FRMPD4

ADK ARID1B C15orf43 CEP290 DCTN5 DQ577902 ELN FUS

ADNP ARID4B C15orf49 CEP41 DCTPP1 DQ578199 EML1 FZD9

ADORA2A ARNT2 C16orf45 CERS4 DCUN1D1 DQ578369 EN2 GABBR1

ADORA3 ARSA C16orf54 CHAT DCX DQ578700 ENO2 GABBR2 ADRB2 ARVCF C16orf92 CHD1L DDC DQ578838 EP300 GABRA1

ADSL ARX C16orf93 CHD2 DDX11 DQ579907 EP400 GABRA2

AF072097 ASH1L C16orf98 CHD7 DDX3X DQ582071 EPC2 GABRA3

AFF2 ASMT C22orf29 CHD8 DDX3Y DQ582073 EPHA6 GABRA4

AGAP1 ASMTL C22orf39 CHL1 DDX53 DQ582448 EPHB2 GABRA5

AGAP2 ASPHD1 C3orf58 CHRFAM7A DDX54 DQ582641 EPHB6

GABRA6

AGBL4 ASS1 C4B CHRM3 DEAF1 DQ582939 EPS8 GABRB1

AGMO ASTN1 CA6 CHRNA7 DEPDC5 DQ583847 ERBB2 GABRB2

AGTR2 ASTN2 CACNA1B CHRNB3 DGCR10 DQ585847 ERBB2IP GABRB3

AHI1 ASXL3 CACNA1C CIB2 DGCR11 DQ586415 ERBB3 GABRD AIFM3 ATG7 CACNA1D CLDN3 DGCR14 DQ586919 ERBB4 GABRE AK023548 ATOH1 CACNA1F CLDN4 DGCR2 DQ587888 ERCC6 GABRG1 AK023809 ATP10A CACNA1G CLDN5 DGCR5 DQ588687 ERG GABRG2 AK056973 ATP1B1 CACNA1H CLIP2 DGCR6L DQ588973 ESR1 GABRG3 AK075019 ATP2A1 CACNA1I CLN3 DGCR8 DQ589249 ESR2 GABRP AK091688 ATP2B2 CACNA2D1 CLSTN3 DGKZ DQ589803 ESRRB GABRQ

AK093758 ATRNL1 CACNA2D2 CLTCL1 DHCR7 DQ590322 ETFB GAD1 AK097453 ATXN2L CACNA2D3 CMIP DIAPH3 DQ590616 ETS1 GAD2 AK097472 ATXN7 CACNB2 CNOT3 DIP2A DQ593032 EXOC3 GALNT13 AK097527 AUTS2 CACNB4 CNR1 DISC1 DQ593239 EXOC5 GALNT14 AK124131 AVPR1A CADM1 CNR2 DKFZP434L187 DQ593342 EXOC6 GALNTL4

AK124673 AX747189 CADM2 CNTN3 DLG4 DQ593983 EXOC6B GAN AK125489 AX747846 CADPS2 CNTN4 DLGAP1 DQ594309 EXT1 GAP43 AK129567 AXL CAMK2G CNTN5 DLGAP2 DQ594669 F13A1 GARNL1 AK302306 BAIAP2 CAMK4 CNTN6 DLGAP3 DQ595055 FABP3 GAS2 
AK302545 BANK1 CAMSAP2 CNTNAP2 DLGAP4 DQ595631 FABP5 GATA4

AK303607 BAZ1B CAMTA1 CNTNAP3 DLL1 DQ595648 FAM135B GATM AK307870 BBS4 CAPN12 CNTNAP4 DLX1 DQ596229 FAM189A1 GBX2 AK309255 BC029255 CAPRIN1 CNTNAP5 DLX2 DQ596303 FAM190A GCH1

AK310030 BC033281 CAPS2 COL25A1 DLX3 DQ596319 FAM57B GDA AK310228 BC035867 CARKD COMT DLX6 DQ596685 FAM91A1 GDI1 AK310526 BC039500 CASC4 CORO1A DMD DQ596686 FAN1 GDPD3 AK311660 BC041003 CASK CREBBP DMPK DQ597560 FARP2 GGNBP2 AKT1 BC041466 CBS CRKL DNAH10 DQ597873 FAT1 GIGYF1 AKT2 BC043570 CBX4 CSDE1 DNAJB1 DQ599532 FBRS GJA1 AKT3 BC050343 CC2D1A CSMD1 DNAJC19 DQ599733 FBXL19 GJA5 ALDH1A3 BC050599 CC2D1B CSNK1E DNAJC30 DQ599785 FBXL19-AS1 GJA8

ALDH5A1 BC070376 CCDC101 CSTF2T DNER DQ600136 FBXO15 GLO1 ALDOA BC073928 CCDC64 CTCF DNHD1 DQ600342 FBXO33 GLRA2 ALOX5AP BC127858 CD163L1 CTF1 DNM1 DQ786262 FBXO40 GLRA3 AMELY BCAP31 CD19 CTNNA3 DNM1L DQ786280 FCRL6 GLRB GLUD2 HDAC6 IL1RAPL2 KMT2C MAPK3 MSR1 NRP2 PDE4A GNAS HDAC9 IL27 KMT2E MAPK7 MTF1 NRXN1 PDE4B GNB1L HDHD1 IMMP2L KPTN MAPK8 MTHFR NRXN2 PDIA3P GOLGA6L1 HDLBP INADL KRT34 MAPK8IP2 MTMR10 NRXN3 PDK1 GOLGA6L2 HEPACAM INO80E LAMA1 MARK1 MTMR12 NSD1 PDXDC1

GOLGA6L7P HERC2 INPP1 LAMB1 MAX MTOR NSF PDZD4 GOLGA8EP HERC2P10 IPW LAMC3 MAZ MTR NTAN1 PDZK1P1 GOLGA8F HERC2P2 IQGAP2 LAT MBD1 MTX2 NTNG1 PECR GOLGA8G HERC2P7 IQSEC2 LAT2 MBD2 MVP NTRK1 PER1 GOLGA8I HERC2P9 ITGA4 LEO1 MBD3 MYH10 NTRK3 PEX7 GOLGA8S HGF ITGA5 LEP MBD3L1 MYH11 NUPR1 PGR GOLGA8T HIRA ITGAL LIMK1 MBD3L2 MYH4 NXF5 PHF15 GPC1 HIRIP3 ITGAM LIN7B MBD3L3 MYLPF NXPH1 PHF2 GPC6 HIST1H1A ITGB3 LINC00035 MBD3L4 MYO16 OCA2 PHF8 GPD2 HIST1H2BB ITGB7 LINC00624 MBD3L5 MYO1A ODF3L2 PHKG2

GPHN HIST1H2BC JARID2 LINC00895 MBD4 MYO9B OFD1 PI4KA GPLD1 HIST1H2BK JB175342 LINC00896 MBD5 MYOC OGT PI4KAP1 GPR139 HIST1H3F JMJD1C LINC00929 MBD6 MYOZ1 OPHN1 PIK3CB GPR37 HIST1H4A JPX LMCD1 MC4R MYT1L OPRM1 PIK3CG GPR6 HIST1H4B JUP LMX1B MCC NAA15 OR1C1 PIK3R2 GPR85 HIST1H4C JX456220 LOC100128714 MCF2 NBEA ORAI2 PIKFYVE

GPR89B HIST1H4D KAL1 LOC100288332 MCPH1 NBPF13P ORAI3 
PINX1

GPR89C HIST1H4F KANK1 LOC100288637 MDGA2 NBPF24 OTUD7A PIR

GPX1 HLA-A KAT2B LOC100289092 MDM2 NBPF8 OTX1 PITX1

GRIA1 HLA-DRB1 KAT8 LOC100289656 MECP2 NCKAP1 OXTR PKD1P1

GRIA2 HMGN1 KATNAL2 LOC100862671 MED13L NCKAP5 P2RX4 PLA1A

GRIA3 HNRNPH2 KCNAB2 LOC23117 MED15 NCKAP5L P2RX6 PLAUR GRIA4 HOMER1 KCND2 LOC283683 MEF2C NCOR1 P2RX6P PLCB1 GRID1 HOXA1 KCNJ10 LOC283685 MET NCOR2 PAFAH1B1 PLCD1 GRID2 HOXB1 KCNJ12 LOC283710 Metazoa_SRP NDE1 PAGR1 PLD1 GRID2IP HRAS KCNJ15 LOC283767 MFRP NDN PAH PLD2 GRIK2 HS3ST5 KCNJ2 LOC284865 MIB1 NDNL2 PAK3 PLN GRIK3 HSD11B1 KCNMA1 LOC388849 MIB2 NDUFA5 PAR1 PLXNA3 GRIN1 HSD3B7 KCNQ2 LOC400891 MIR1286 NEFL PAR4 PLXNA4 GRIN2A HSP90AA1 KCNQ3 LOC440243 MIR1306 NELL1 PAR5 PLXNB3 GRIN2B HSPH1 KCNT1 LOC440354 MIR137 NF1 PARD3 PNPLA4 GRIN2C HTR1A KCTD13 LOC595101 MIR185 NFATC2IP PARD3B POGZ GRIN2D HTR1B KCTD4 LOC606724 MIR211 NFIA PARK2 POM121L4P GRIN3A HTR1D KDM3A LOC613037 MIR3179-2 NHLRC1 PAR-SN POMGNT1

GRIN3B HTR1E KDM4B LOC613038 MIR3179-3 NIPA1 PASK PON1 GRIP1 HTR1F KDM5B LOC643699 MIR3180-3 NIPA2 PAX5 POT1 GRM1 HTR2A KDM5C LOC646278 MIR3180-4 NIPBL PCDH10 PPFIA1 GRM2 HTR2B KDM5D LOC729444 MIR3618 NLGN1 PCDH11X PPM1D GRM3 HTR2C KDM6B LOC730183 MIR4284 NLGN2 PCDH11Y PPP1R1B GRM4 HTR3A KHDRBS2 LPL MIR4508 NLGN3 PCDH15 PPP1R3F GRM5 HTR3B KIAA0100 LRFN5 MIR4509-1 NLGN4X PCDH19 PPP2R5D GRM6 HTR3C KIAA0182 LRP2 MIR4517 NLGN4Y PCDH8 PPP3CB GRM7 HTR3D KIAA0232 LRP2BP MIR4518 NOMO1 PCDH9 PPP4C GRPR HTR3E KIAA0430 LRPPRC MIR4519 NOMO3 PCDHA1 PRICKLE1 GSC2 HTR4 KIAA1586 LRRC1 MIR4715 NONO PCDHA10 PRICKLE2 GSDMC HTR5A KIAA1653 LRRC14 MIR4721 NOS1 PCDHA11 PRKAA1 GSK3B HTR6 KIAA2022 LRRC7 MIR4761 NOS1AP PCDHA12 PRKAA2 GSN HUWE1 KIF13B LSAMP MIR484 NOS2A PCDHA13 PRKAB2 GSS HV593096 KIF1A LZTR1 MIR5087 NPAP1 PCDHA2 PRKCA GSTM1 HV593110 KIF22 LZTS2 Mir_548 NPAS2 PCDHA3 PRKCB GTF2I HV593127 KIF4A MACROD2 MIR590 NPIP PCDHA4 PRKD1 GTF2IRD1 HV593134 KIF5C MAFK Mir_649 NPIPL1 PCDHA5 PRKY GUCY1A2 HV593135 KIRREL3 MAGED1 MIR762 NPIPL3 PCDHA6 PRODH

HAUS7 HV593178 KIT MAGEL2 MKL2 NPY1R PCDHA7 PRPF39 HBT8 HV593183 KLC1 MAL MKRN3 NPY5R PCDHA8 PRR14 HCFC1 HYDIN KLC2 MAOA MLST8 NR3C2 PCDHA9 PRRG4 
HCN1 ICA1 KLF13 MAOB MLXIPL NRCAM PCDHAC1 PRRT2 hCPE-R IKBKG KLHL13 MAP2 MPP6 NRG1 PCDHAC2 PRSS36 HDAC1 IL16 KLHL22 MAPK1 MPV17L NRG2 PCDHGA11 PRSS38 HDAC2 IL1R2 KMO MAPK11 MRPL40 NRG3 PCOLCE PRSS53 HDAC4 IL1RAPL1 KMT2A MAPK12 MSN NRG4 PDE1C PRSS8 PRUNE2 RIMS1 SHANK3 SND1 SNORD116-5 TBC1D10B TRPC1 VPS13B PRY RIMS3 SIN3A SNORA30 SNORD116-8 TBC1D23 TRPC3 VPS37D PSD3 RNF216P1 SIX2 SNORD109B SNRPN TBC1D5 TRPC4 VSIG4 PSMB1 RNF40 SKI SNORD115-1 SNTG2 TBC1D7 TRPC5 WAC PSMC2 RNPS1 SLC16A7 SNORD115-10 SNURF TBCK TRPC6 WBP5 PSMD10 ROBO1 SLC17A1 SNORD115-11 SNURF-SNRPN TBL1X TRPM1 WBSCR22

PTCHD1 ROBO2 SLC17A2 SNORD115-13 SNX19 TBL1XR1 TSC1 WBSCR27

PTEN RORA SLC17A6 SNORD115-14 SNX29P2 TBL2 TSC2 WBSCR28 PTGER3 RPL10 SLC17A7 SNORD115-15 SNX9 TBP TSN WDFY3 PTGS2 RPP25 SLC17A8 SNORD115-16 SOD1 TBR1 TSPAN6 WDR55 PTPN11 RPS4Y1 SLC18A1 SNORD115-17 SOD2 TBX1 TSPAN7 WDR93 PTPRB RPS6KA2 SLC18A2 SNORD115-2 SOX1 TBX6 TSPYL2 WHAMMP2 PTPRC RPS6KA3 SLC18A3 SNORD115-22 SOX5 TCF20 TSSK2 WHAMMP3

PTPRD RPTOR SLC1A1 SNORD115-24 SOX9 TCF3 TTI2 WHSC1 PTPRM RRN3 SLC1A2 SNORD115-25 SPARCL1 TCF4 TTLL3 WNK3 PTPRT RRN3P2 SLC1A4 SNORD115-26 SPAST TCTE3 TTTY10 WNT1 PWRN1 RTN4R SLC1A6 SNORD115-27 SPATA13 TDO2 TTTY14 WNT2 PWRN2 S100G SLC1A7 SNORD115-28 SPN TENM1 TTTY15 WT1 PXDN SAE1 SLC22A15 SNORD115-3 SPNS1 TGM1 TUBA3FP XIST PYCARD SAP130 SLC24A2 SNORD115-30 SRCAP TH TUBGCP5 XPC PYDC1 SAP30 SLC25A1 SNORD115-31 SRD5A2 THAP7 TUFM XPO1 QPRT SATB2 SLC25A12 SNORD115-32 SREBF1 THAP7-AS1 TXLNG YEATS2

QRICH1 SBF1 SLC25A14 SNORD115-33 SRPK2 THAP8 TXLNG2P YPEL3 RAB11FIP5 SCAMP5 SLC25A24 SNORD115-35 SRY THBS1 TXNRD2 Y_RNA

RAB19 SCARA3 SLC25A27 SNORD115-37 ST7 THRA TYR YTHDC1 RAB2A SCARF2 SLC29A4 SNORD115-38 ST8SIA2 TIGD4 U84523 YTHDC2

RAB39B SCFD2 SLC30A5 SNORD115-39 STAU2 TIMP1 UBA6 YWHAE RAB3A SCN1A SLC32A1 SNORD115-4 STK39 TJP1 UBE2A ZBTB16 RAB3IL1 SCN2A SLC35A3 SNORD115-40 STS TLK2 UBE2H ZBTB20 RAB5A SCN5A SLC38A1 SNORD115-41 STX1A TLS/FUS-ERG UBE3A ZDHHC8

RABEP2 SCN7A SLC38A10 SNORD115-44 STX1B TM4SF20 UBE3B ZFX RAC1 SCN8A SLC39A11 SNORD115-45 STX4 TMEM191A UBE3C ZFY 
RAC3 SCRIB SLC4A10 SNORD115-47 STXBP1 TMEM191B UBN2 ZG16 RAI1 SDC2 SLC6A1 SNORD115-48 STXBP5 TMEM219 UBR1 ZMYND11 RANBP1 SDK1 SLC6A11 SNORD115-5 STXBP6 TMEM231 UBR3 ZNF18 RANBP17 SEMA3A SLC6A12 SNORD115-6 SULT1A1 TMLHE UBR5 ZNF292

RAP1GDS1 SEMA5A SLC6A13 SNORD115-7 SULT1A2 TMLHE-AS1 UBR7 ZNF385B

RAPGEF4 SEPHS2 SLC6A2 SNORD115-8 SULT1A4 TMSB4Y UFD1L ZNF407

RASD1 SEPT1 SLC6A3 SNORD115-9 SUV420H1 TMX2 ULK4P1 ZNF433 RASSF5 SEPT5-GP1BB SLC6A4 SNORD116-1 SVIL TNIP2 ULK4P3 ZNF48 RB1CC1 SERPIND1 SLC6A8 SNORD116-10 SYAP1 TNK2 UPB1 ZNF517 RbBP7 SERPINE1 SLC7A4 SNORD116-11 SYN1 TNN UPF2 ZNF559 RBFOX1 SETBP1 SLC7A5P1 SNORD116-12 SYN2 TNRC6B UPF3B ZNF629 RBM8A SETD1A SLC9A6 SNORD116-13 SYN3 TOMM20 USP41 ZNF646 RBMS3 SETD2 SLC9A9 SNORD116-15 SYNCRIP TOP1 USP9X ZNF668 RBMX SETD5 SLCO1B1 SNORD116-16 SYNE1 TOP3B USP9Y ZNF688 RBMX2 SETDB1 SLCO1B3 SNORD116-18 SYNGAP1 TPO UTP6 ZNF689 RBMY1B SETDB2 SLIT3 SNORD116-19 SYNJ2 TPRX1 UTRN ZNF713 RBMY1F SEZ6L2 SLITRK5 SNORD116-2 SYP TRIM17 UTY ZNF74 REEP3 SF1 SLX1A SNORD116-20 SYT1 TRIM33 VAMP1 ZNF747 RELN SGSH SLX1B-SULT1A4 SNORD116-22 SYT11 TRIM72 VAMP2 ZNF764

RER1 SGSM3 SMARCC2 SNORD116-23 SYT17 TRIO VASH1 ZNF768 RERE SH2B1 SMC2 SNORD116-24 SYT3 TRIP12 VCP ZNF771 RFC2 SH3GL3 SMCX SNORD116-25 SYT4 TRMT2A VCX ZNF774 RFWD2 SH3KBP1 SMCY SNORD116-26 TAAR2 TRNA_Asn VCX2 ZNF778

RGS7 SHANK1 SMG6 SNORD116-28 TAF1C TRNA_Gln VGF ZNF785 RHEB SHANK2 SMURF1 SNORD116-29 TAF4 TRNA_Glu VIL1 ZNF8 RHOXF1 SHANK2-AS1 SNAP25 SNORD116-3 TANGO2 TRNA_His VIP ZNF804A

RIBC1 SHANK2-AS3 SNAP29 SNORD116-4 TAOK2 TRNA_Pseudo VKORC1 ZNF827

RIMBP3

\subsection{Attachment 4}

AAK1 ARID1A CALM3 DDN FAM190B HK1 LLGL1 MYO5A AATK ARID1B CAMK2A DDX24 FAM21A HMGXB3 LMTK2 MYST3 ABCA2 ARID2 CAMK2B DENND5A FAM5B HNRNPUL1 LMTK3 MYT1L 
ABCA3 ARNT2 CAMK2N1 DGCR2 FAM65A HSP90AB1 LPHN1 NACAD ABCG1 ARPP21 CAMKK2 DGKZ FAM91A1 HTT LPHN3 NAT8L ABR ARRB1 CAMSAP1 DHX30 FASN HUWE1 LPIN2 NAV1 ACCN1 ARVCF CAMSAP1L1 DICER1 FAT1 IDS LPPR4 NAV2 ACLY ASH1L CAMTA1 DIDO1 FAT2 IGSF9B LRP1 NAV3 ACO2 ATF7IP CAMTA2 DIP2A FAT3 INPP4A LRP3 NBEA ACTB ATG2A CAND1 DIP2B FAT4 INTS1 LRP8 NCAM1 ADAP1 ATG2B CASKIN1 DIP2C FBXL16 IPO13 LRRC41 NCAN ADARB1 ATG9A CBX6 DIRAS2 FBXL19 IPO4 LRRC4B NCDN ADCY1 ATMIN CBX6-NPTXR DISP2 FBXO41 IPO5 LRRC68 NCKAP1 ADCY5 ATN1 CDC42BPA DLC1 FCHO1 IQSEC2 LRRC7 NCOA1 ADD1 ATP13A2 CDC42BPB DLG2 FKBP8 IQSEC3 LRRC8B NCOA2 ADNP ATP1A1 CDK16 DLG4 FOXK2 IRS2 LRRN2 NCOA6 ADRBK1 ATP1A2 CDK17 DLG5 FOXO3 ITPR1 LYNX1 NCOR1 AFF3 ATP1A3 CDK5R1 DLGAP1 FRMPD4 ITSN1 MACF1 NCOR2 AFF4 ATP1B1 CDK5R2 DLGAP2 FRY JAK1 MADD NCS1 AGAP1 ATP2A2 CDKL5 DLGAP3 FSCN1 JPH3 MAGED1 NDRG2 AGAP2 ATP2B2 CELF5 DLGAP4 FYN JPH4 MAGI2 NDRG4 AGAP3 ATP2B4 CELSR2 DMWD GABBR1 KALRN MAN2A2 NDST1 AGPAT3 ATP5A1 CELSR3 DMXL2 GABBR2 KCNA2 MAP1A NEDD4 AGRN ATP5B CHD3 DNAJC6 GARNL3 KCNB1 MAP1B NELF AGTPBP1 ATP6V0A1 CHD4 DNM1 GAS7 KCNC3 MAP2 NEURL AHDC1 ATP6V0D1 CHD5 DOCK3 GBF1 KCND2 MAP3K12 NEURL4 AKAP6 ATP6V1B2 CHD6 DOCK4 GCN1L1 KCNH1 MAP4 NF1 AKAP9 ATP9A CHD8 DOCK9 GIT1 KCNH3 MAP4K4 NFIC AKT3 ATXN1 CHN1 DOPEY1 GLUL KCNH7 MAP7D1 NFIX ALDOA AUTS2 CHN2 DOPEY2 GM996 KCNMA1 MAPK1 NGEF ALDOC B3GAT1 CHST2 DOT1L GNAL KCNQ2 MAPK4 NHSL1 ALS2 BAI1 CIC DPP8 GNAO1 KCNQ3 MAPK8IP1 NISCH AMPH BAI2 CIT DPYSL2 GNAS KCNT1 MAPK8IP3 NLGN2 ANAPC1 BAP1 CKAP5 DSCAM GNAZ KDM4B MAPKBP1 NLGN3 ANK1 BAT2 CKB DSCAML1 GNB1 KDM5C MAST1 NOMO1 ANK2 BAT2L1 CLASP1 DST GPAM KDM6B MAST2 NPAS2 ANK3 BAT2L2 CLASP2 DTNA GPM6A KIAA0090 MAST4 NPTXR ANKRD11 BAT3 CLCN3 DTX1 GPR158 KIAA0100 MAZ NR2F1 ANKRD17 BAZ2A CLEC16A DUSP8 GPR162 KIAA0226 MBD5 NRGN ANKRD52 BCAN CLIP3 DYNC1H1 GPRIN1 KIAA0284 MBP NRIP1 AP1B1 BCL9L CLSTN1 EEF1A2 GRAMD1B KIAA0317 MED13 NRXN1 AP2A1 BCR CLTC EEF2 GRIK3 KIAA0430 MED13L NRXN2 AP2A2 BIRC6 CNP EGR1 GRIK5 KIAA0664 MED14 NRXN3 AP2B1 BMPR2 COBL EHMT1 GRIN1 KIAA0802 MED16 NSD1 AP3D1 BPTF COPG EHMT2 GRIN2A KIAA0913 MEF2D NSF APBA1 BRD4 CPE EIF2C1 GRIN2B KIAA0947 MFHAS1 NTRK2 APBB1 BRSK1 CPLX1 EIF2C2 GRLF1 KIAA1045 MGAT5B NTRK3 
APC BRSK2 CPLX2 EIF4G1 GRM4 KIAA1109 MIB1 NUP98 APC2 BSN CPT1C EIF4G2 GRM5 KIAA1244 MICAL2 NWD1 APLP1 BZRAP1 CREBBP EIF4G3 GSK3B KIAA1688 MINK1 ODZ2 APOE C11ORF41 CRMP1 ELFN2 GTF3C1 KIAA2018 MKL2 ODZ3 APP C14ORF4 CRTC1 ELMO2 GTF3C2 KIF1A MLL ODZ4 ARAP2 C19ORF26 CTBP1 EML2 HCFC1 KIF1B MLL2 OGDH ARF3 C20ORF117 CTNNB1 ENC1 HCN2 KIF21A MLL3 OLFM1 ARFGEF1 C20ORF12 CTNND2 EP300 HDAC4 KIF21B MLL5 OXR1 ARHGAP20 C2CD2L CUL9 EP400 HDAC5 KIF3C MMP24 PACS1 ARHGAP21 CABIN1 CUX1 EPB41L1 HDLBP KIF5A MON2 PACS2 ARHGAP23 CACNA1A CUX2 EPB49 HEATR5B KIF5C MPRIP PAK6 ARHGAP32 CACNA1B CYFIP2 EPHA4 HERC1 KIFC2 MTMR4 PCDH1 ARHGAP33 CACNA1E DAB2IP EPN1 HERC2 KLC1 MTOR PCDH10 ARHGEF11 CACNA1G DAGLA EXTL3 HIPK1 KLHL22 MTSS1L PCDH7 ARHGEF12 CACNA1I DAPK1 FAM115A HIPK2 KNDC1 MYCBP2 PCDH9

ARHGEF17 CACNB1 DBC1 FAM120A HIPK3 LARGE MYH10 PCDHA4 ARHGEF2 CACNB3 DCAF6 FAM160A2 HIVEP1 LARS2 MYO10 PCDHAC2

ARHGEF4 CADPS DCLK1 FAM171B HIVEP2 LHFPL4 MYO16 PCDHGA12

ARHGEF7 CALM1 DCTN1 FAM179B HIVEP3 LINGO1 MYO18A PCDHGC3

PCLO POLR2A RALGAPB SCD2 SMARCC2 SYNGR1 TRIM37 USP22 PCNX PPARGC1A RALGDS SCN2A SMG1 SYNJ1 TRIM9 USP32 PCNXL2 PPFIA3 RAP1GAP SCN8A SMPD3 SYNPO TRIO USP34 PCNXL3 PPM1E RAP1GAP2 SEC14L1 SNAP25 SYT1 TRIP12 USP5 PDE2A PPP1R9B RAPGEF1 SEC16A SNAP91 SYT7 TRO USP9X PDE4B PPP2R1A RAPGEF2 SEC23A SNPH TANC2 TRPC4AP VAMP2 PDE4DIP PPP2R2C RAPGEF4 SECISBP2L SOBP TAOK1 TRPM3 VPS13D PDE8B PPP2R5B RAPGEFL1 SEPT3 SON TAOK2 TRRAP VPS41 PDS5B PPP3CA RASGRF1 SEPT5 SORBS2 TBC1D9 TSC2 WASF1 PDZD2 PREX1 RASGRP1 SETD5 SORL1 TBC1D9B TSC22D1 WDFY3 PDZD8 PREX2 RC3H1 SETX SORT1 TCF20 TSHZ1 WDR13 PEG3 PRICKLE2 RC3H2 SEZ6L2 SPAG9 TCF25 TSPAN7 WDR6 PER1 PRKACB RELN SGIP1 SPARCL1 TCF4 TSPYL4 WNK1 PFKM PRKCB RERE SGSM2 SPEG TEF TTBK1 WNK2 PGM2L1 PRKCE REV3L SH3BP4 SPEN THRA TTBK2 WWC1 PHACTR1 PRKCG RGS7BP SHANK1 SPHKAP TIAM1 TTC3 XPO6 PHF12 PROSAPIP1 RHOB SHANK2 SPIRE1 TLE3 TTC7B XPO7 PHF20 PRPF8 RHOBTB2 SHANK3 SPRED1 TLN2 TTLL7 YWHAG PHLDB1 PRR12 RICH2 SIPA1L1 SPRN TMEM132A TTYH1 ZC3H4 PHYHIP PSAP RIMBP2 SIPA1L2 SPTAN1 TMEM151A TTYH3 ZC3H7B PI4KA PSD RPH3A SIPA1L3 SPTB TMEM151B TUBB3 ZCCHC14 
PIGQ PTCH1 RPRD2 SKI SPTBN1 TMEM63B TUBB4 ZEB2 PIKFYVE PTEN RPTOR SLC12A5 SPTBN2 TMEM8B TULP4 ZER1 PINK1 PTK2 RTN1 SLC12A6 SRCIN1 TMOD2 UBA1 ZFHX2 PIP5K1C PTK2B RTN3 SLC17A7 SREBF2 TNIK UBAP2L ZFP106 PITPNM1 PTPN11 RTN4 SLC1A2 SRGAP3 TNK2 UBE2O ZFR PITPNM2 PTPN5 RTN4R SLC22A17 SRRM2 TNKS UBE3B ZFYVE1 PJA2 PTPRD RUSC1 SLC24A2 STK25 TNPO2 UBE3C ZHX3 PKD1 PTPRF RUSC2 SLC25A23 STOX2 TNRC18 UBQLN1 ZMIZ1 PKP4 PTPRG RYR2 SLC4A3 STRN4 TNRC6B UBQLN2 ZMIZ2 PLCB1 PTPRJ SALL2 SLC4A4 STXBP1 TNS3 UBR3 ZNF238 PLCH2 PTPRN2 SAMD4B SLC4A8 STXBP5 TPPP UBR5 ZNF365 PLD3 PTPRS SAP130 SLC6A1 SUPT6H TRAK1 UHRF1BP1L ZNF462 PLEC PTPRT SAPS1 SLC6A17 SV2A TRAK2 ULK1 ZNF521 PLP1 PUM1 SAPS2 SLC8A1 SV2B TRAPPC10 ULK2 ZNF536 PLXNA1 PUM2 SASH1 SLC8A2 SYMPK TRIL UNC13A ZNF704 PLXNA2 QKI SBF1 SLITRK5 SYN1 TRIM2 UNC13C ZNF827 PLXNA4 R3HDM1 SCAF1 SMARCA2 SYNE1 TRIM3 UNC5A ZNFX1 PLXNB1 R3HDM2 SCAP SMARCA4 SYNGAP1 TRIM32 UQCRC1 ZYG11B

PLXND1 RALGAPA1

\subsection{Attachment 5}

ABI2 CCDC88C DNAJC6 ILF2 NAA15 PLXNA3 SLC6A1 TCF4 ADCY5 CDC42BPB DOCK8 IQGAP3 NAT8L PLXNB1 SLC6A10P TCF7L2 ADCY9 CDC45 DSCAM IQSEC2 NAV2 POGZ SLC6A8 TNRC18 ADGRL2 CDH10 DVL3 ITPR1 NBEA PPM1D SLC9A3 TNRC6B ADNP CDKL5 DYRK1A KAT2B NCKAP1 PPP2R5D SLITRK5 TRIO AGAP2 CHD1 EPHB2 KATNAL2 NCOR1 PROX2 SMAD2 TRIP12 AGO4 CHD2 ERBIN KCNH1 NEMF PSEN2 SMAD4 TRRAP AHNAK CHD4 FAM8A1 KCNH3 NFIA PTEN SMARCC2 TSC2 AK8 CHD7 FOXP1 KIRREL3 NISCH PTK7 SMC3 TUBA1A ALdH5A1 CHD8 FOXP3 KMT2A NLGN3 PTPN11 SPAST UBN2 ANK2 CHRNA7 GABRB3 KMT2C NLGN4X RAB2A SPP2 UIMC1 ANK3 COL4A3BP GALNT18 KMT2E NR2E3 RALGAPB SRCAP UNC80 ANKRD11 CREBBP GIGYF2 KMT5B NRXN1 RANBP2 SRGAP2 VCP ANP32A CSMD1 GLI2 LAMC3 NSD2 RELN SRGAP3 WAC APH1A CSTF2T GPRIN1 LHX1 NTM RIMBP2 SRM WDFY3 ARHGAP30 CTNNB1 GPS1 LZTR1 NTNG1 RIMS1 ST3GAL6 WDR45 ARHGAP32 CTNND2 GRB10 MAGEL2 NUAK1 RNF38 STAG1 WNT7B ARHGAP5 CTTNBP2 GRIA2 MAP3K1 PARD3B RPL19 STXBP1 WNT9A ARID1B CUL3 GRIN1 MECP2 PASK SCN1A STXBP5 YES 
ARIH1 CUL7 GRIN2A MED13L PAX5 SCN2A SUPT16H YTHDC1 ARVCF DCX GRIN2B MFRP PCOLCE SETBP1 SVIL ZBTB45 ARX DDX3X GTPBP4 MIP PHF2 SETD2 SYNCRIP ZC3H4 ASH1L DHX9 HDAC9 MKI67 PHF3 SETD5 SYNGAP1 ZFHX3 ASXL3 DIP2A HECTD1 MOV10 PHF7 SHANK1 TANC2 ZMYM2 CACNA2D3 DLG2 HIVEP3 MYH9 PHIP SHANK2 TBL1XR1 ZMYND11 CAPRIN1 DLG4 HUWE1 MYT1L PIK3CA SIN3A TBR1 ZNF292 CASK DLGAP1

\subsection{Attachment 6}

ABAT ALDH5A1 GABRA5 GABRR2 GNB1 GNG7 KCNJ2 SLC6A1 ADCY1 ARHGEF9 GABRA6 GABRR3 GNB2 GNG8 KCNJ3 SLC6A11 ADCY2 CPLX1 GABRB1 GAD1 GNB3 GNGT1 KCNJ4 SLC6A12 ADCY3 DNAJC5 GABRB2 GAD2 GNG10 GNGT2 KCNJ5 SLC6A13 ADCY4 GABBR1 GABRB3 GNAI1 GNG12 HSPA8 KCNJ6 SNAP25 ADCY5 GABBR2 GABRG2 GNAI2 GNG2 KCNJ10 KCNJ9 STX1A ADCY6 GABRA1 GABRG3 GNAI3 GNG3 KCNJ12 RAB3A STXBP1 ADCY7 GABRA2 GABRQ GNAL GNG4 KCNJ15 RIMS1 SYT1 ADCY8 GABRA3 GABRR1 GNAT3 GNG5 KCNJ16 SLC32A1 VAMP2 ADCY9 GABRA4

\subsection{Attachment 7}

AASS CRYGD GADD45G KBTBD11 MKRN1 PHLDA1 RPS11 TAF7 ACAT2 CTU1 GCA KCNK12 MRPS10 PNISR RPS26P15 TCP1 AGBL5 CYYR1 GDF10 KDR MTM1 PODXL SALL3 TDP2 ALDH7A1 DCUN1D2 GNB3 KIT MYEF2 POLR3G SCPEP1 TENM3 ALOX5 DCUN1D4 GPR27 KLF4 MYL12A POP4 SEMA6A TFG ARPC5 DDIT4L GUF1 KRR1 MYL12B PRKDC SF3B6 TLE3 ATOH1 DHX9 HERC2 L1TD1 NACC1 PSMA7 SGCB TMEM126A ATP5G2 DLX5 HIST1H2AE LAMA1 NAP1L1 PYGL SLC6A1 TMEM141 BAGE2 DLX6 HIST1H2BG LAMP1 NDUFA8 RAB6B SLC6A6 TMEM261 BEND4 DNMT3B HIST1H3G LGALS8 NFE2L3 RAD21 SMCO4 TMEM63B BEND5 DUSP23 HIST1H4C LHPP P3H3 RAPGEF5 SNORA18 TRIM41 C12orf57 E2F5 HIST1H4F LHX1 PAPSS1 RBM28 SNRPB2 TWIST1 C14orf1 EIF4A3 HIST1H4I LIN7C PCDH8 RBP4 SP5 U2AF1 CACHD1 EIF4EBP3 HMGB3 LINC00094 PCDHB5 RHOB SPATA18 UBE2H

CADPS2 EMBP1 HMGN3 LMO1 PCNP RIDA SPSB2 UCHL1 
CAND2 ENO1 HNRNPA2B1 LONRF1 PCTP RMRP SRPRB USP1 CCDC174 FAM189A1 HNRNPF LRIG1 PDCD10 RNF126P1 SRSF9 VRTN CCKBR FEZF1 HSPD1 LRRCC1 PDCD11 RPL13A SSR1 XKR7 CDCA7 FGF19 HTR1B LSM4 PDXP RPL13P5 STC2 ZFAND4 COX5A FGF4 ID4 LTA4H PHAX RPL22 SUPT6H ZFP42 CPZ FOS IFI6 MAPRE1 PHGDH RPRM SYT17 ZNF488 CRABP1 FZD5 IGDCC4

\subsection{Attachment 8}

AKT1 EEF2K LAMTOR1 MLST8 PRKAB1 RHEB RRAGB STRADB AKT1S1 EIF4B LAMTOR2 MTOR PRKAB2 RPS6 RRAGC TSC1 AKT2 EIF4E LAMTOR3 PPM1A PRKAG1 RPS6KB1 RRAGD TSC2 CAB39 EIF4EBP1 LAMTOR4 PRKAA1 PRKAG2 RPTOR STK11 YWHAB CAB39L EIF4G1 LAMTOR5 PRKAA2 PRKAG3 RRAGA STRADA

\subsection{Attachment 9}

ACTB DUSP5 FGFR3 IRS4 NRTN PSMA2 PSMD8 RRAGB ACTG1 DUSP6 FGFR4 ITGA2B PAQR3 PSMA3 PSMD9 RRAGC ACTN2 DUSP7 FGG ITGB3 PDE3B PSMA4 PSME1 RRAGD AKAP9 DUSP8 FN1 JAK1 PDGFA PSMA5 PSME2 SHC1 AKT1 DUSP9 FRS2 JAK2 PDGFB PSMA6 PSME3 SHC2 AKT1S1 EEF2K FRS3 JAK3 PDGFRA PSMA7 PSME4 SHC3 AKT2 EGF FYN KBTBD7 PDGFRB PSMA8 PSMF1 SOS1 ANGPT1 EGFR GAB1 KIT PDPK1 PSMB1 PSPN SPRED1 APBB1IP EIF4B GDNF KITLG PEA15 PSMB10 PTK2 SPRED2 ARAF EIF4E GFRA1 KL PEBP1 PSMB11 PTPN11 SPRED3 ARRB1 EIF4EBP1 GFRA2 KLB PHB PSMB2 PTPRA SPTA1 ARRB2 EIF4G1 GFRA3 KRAS PIK3C3 PSMB3 RAF1 SPTAN1 ARTN ERBB2 GFRA4 KSR1 PIK3CA PSMB4 RAP1A SPTB BRAF ERBB3 GRB2 KSR2 PIK3CB PSMB5 RAP1B SPTBN1 BRAP ERBB4 GRIN1 LAMTOR1 PIK3R1 PSMB6 RAPGEF2 SPTBN2 BTC EREG GRIN2A LAMTOR2 PIK3R2 PSMB7 RASA1 SPTBN4 CAB39 FGA GRIN2B LAMTOR3 PIK3R4 PSMB8 RASA2 SPTBN5 CAB39L FGB GRIN2C LAMTOR4 PPM1A PSMB9 RASA3 SRC CALM1 FGF1 GRIN2D LAMTOR5 PPP2CA PSMC1 RASA4 STK11 CAMK2A FGF10 HBEGF LAT PPP2CB PSMC2 RASAL1 STRADA CAMK2B FGF16 HRAS MAP2K1 PPP2R1A PSMC3 RASAL2 STRADB CAMK2D FGF17 IGF1 MAP2K2 PPP2R1B PSMC4 RASAL3 SYNGAP1 
CAMK2G FGF18 IGF1R MAP3K11 PPP2R5A PSMC5 RASGEF1A TEK CNKSR1 FGF19 IGF2 MAPK1 PPP2R5B PSMC6 RASGRF1 THEM4 CNKSR2 FGF2 IL17RD MAPK3 PPP2R5C PSMD1 RASGRF2 TLN1 CSF2 FGF20 IL2 MARK3 PPP2R5D PSMD10 RASGRP1 TLR9 CSF2RA FGF22 IL2RA MLST8 PPP2R5E PSMD11 RASGRP3 TRIB3 CSF2RB FGF23 IL2RB MTOR PPP5C PSMD12 RASGRP4 TSC1 CSK FGF3 IL2RG NCAM1 PRKAA1 PSMD13 RBX1 TSC2 CUL3 FGF4 IL3 NEFL PRKAA2 PSMD14 RET UBA52 DAB2IP FGF5 IL3RA NF1 PRKAB1 PSMD2 RHEB UBB DLG4 FGF6 IL5 NRAS PRKAB2 PSMD3 RPS27A UBC DUSP1 FGF7 IL5RA NRG1 PRKAG1 PSMD4 RPS6 VCL DUSP10 FGF8 IQGAP1 NRG2 PRKAG2 PSMD5 RPS6KB1 VWF DUSP16 FGF9 IRS1 NRG3 PRKAG3 PSMD6 RPTOR WDR83 DUSP2 FGFR1 IRS2 NRG4 PSMA1 PSMD7 RRAGA YWHAB DUSP4 FGFR2

\subsection{Attachment 10}

A1BG ACADS ADAM9 AHCY AMT AP2M1 ARHGAP24 ARRB1

A1CF ACADSB ADAMTS10 AHCYL1 ANAPC13 AP3B2 ARHGAP26 ARRB2

A4GALT ACAP2 ADAMTS14 AHCYL2 ANAPC2 AP3D1 ARHGAP28 ARRDC3

AADAC ACAP3 ADAMTS17 AHNAK ANAPC4 AP3M1 ARHGAP29 ARRDC5

AADACL2 ACAT1 ADAMTS18 AHR ANAPC5 AP3M2 ARHGAP32 ARSJ

AADACL4 ACAT2 ADAMTS19 AHSA1 ANGEL2 AP3S2 ARHGAP33 ARSK

AADAT ACBD5 ADAMTS2 AHSA2 ANGPT1 AP4B1 ARHGAP35 ARVCF AAK1 ACBD6 ADAMTS20 AIDA ANGPT2 AP4E1 ARHGAP36 ASAP1

AANAT ACCSL ADAMTS3 AIFM1 ANGPTL1 AP4M1 ARHGAP39 ASAP2

AAR2 ACD ADAMTS6 AIFM2 ANGPTL2 AP4S1 ARHGAP44 ASAP3 AARS ACE ADAMTS9 AIFM3 ANGPTL3 AP5Z1 ARHGAP5 ASB1 AARSD1 ACHE ADAMTSL1 AIG1 ANK1 APAF1 ARHGAP6 ASB13 AASDHPPT ACIN1 ADAP2 AIMP2 ANK2 APBA1 ARHGAP9 ASB14 AASS ACLY ADAR AIP ANK3 APBA2 ARHGDIA ASB2 AATF ACMSD ADARB1 AIRE ANKDD1A APBB1 ARHGDIB ASB4 ABAT ACOT7 ADARB2 AK1 ANKFY1 APBB1IP ARHGDIG ASB5 ABCA1 ACOX2 ADCK3 AK4 ANKH APBB2 ARHGEF1 ASB6 ABCA12 ACOX3 ADCY1 AK5 ANKHD1 APC ARHGEF10L ASB7 ABCA2 ACPL2 ADCY3 AK7 ANKHD1-EIF4EBP3 APCDD1 ARHGEF11 
ASB8

ABCA3 ACPP ADCY4 AKAP17A ANKIB1 APCS ARHGEF12 ASB9 ABCA7 ACPT ADCY5 AKAP8L ANKLE2 APEH ARHGEF17 ASF1B ABCB10 ACR ADCY6 AKR1B1 ANKMY1 APEX2 ARHGEF3 ASGR1 ABCB4 ACRBP ADCY7 AKR1C1 ANKRA2 API5 ARHGEF4 ASGR2 ABCB6 ACRC ADCY8 AKR1C2 ANKRD11 APITD1-CORT ARHGEF40 ASH1L

ABCB9 ACSBG1 ADCY9 AKR1D1 ANKRD12 APLNR ARHGEF9 ASH2L ABCC1 ACSF2 ADCYAP1 AKT1S1 ANKRD13A APLP1 ARID1A ASIC1 ABCC10 ACSF3 ADCYAP1R1 AKT2 ANKRD13B APLP2 ARID1B ASIC2 ABCC5 ACSL1 ADD2 AKT3 ANKRD13C APOA1 ARID2 ASIC5 ABCC8 ACSL4 ADH1A ALAD ANKRD17 APOA1BP ARID3A ASNA1 ABCC9 ACSL6 ADH6 ALAS1 ANKRD27 APOBEC3C ARID3B ASPA ABCD1 ACSM4 ADHFE1 ALB ANKRD28 APOBEC3D ARID4A ASPH ABCD2 ACSS2 ADI1 ALDH1A1 ANKRD29 APOC1 ARID4B ASPHD1 ABCD3 ACSS3 ADIPOR1 ALDH1A2 ANKRD32 APOL6 ARID5A ASPHD2 ABCE1 ACTA1 ADK ALDH1A3 ANKRD34A APOOL ARIH1 ASPRV1 ABCF1 ACTA2 ADNP ALDH1L1 ANKRD39 APPBP2 ARIH2 ASPSCR1 ABCF2 ACTB ADNP2 ALDH2 ANKRD40 APRT ARL10 ASRGL1 ABCF3 ACTC1 ADORA1 ALDH3A2 ANKRD44 AQP2 ARL14EP ASS1 ABCG1 ACTG1 ADORA2A ALDH6A1 ANKRD49 AQP3 ARL15 ASTN2 ABCG2 ACTG2 ADORA3 ALDH7A1 ANKRD5 AQP4 ARL2 ASUN ABCG4 ACTL6B ADPRH ALDH8A1 ANKRD50 AQP5 ARL3 ASXL3 ABHD1 ACTL7A ADPRHL2 ALDOA ANKRD52 AQP9 ARL4A ASZ1 ABHD11 ACTL7B ADRA1B ALDOC ANKS1A AR ARL4D ATAD1 ABHD12 ACTL8 ADRA2B ALG10 ANKS1B ARAF ARL5B ATAD5 ABHD13 ACTN1 ADRBK2 ALG11 ANKS6 ARAP1 ARL6 ATAT1 ABHD15 ACTN2 ADSL ALG12 ANO1 ARAP2 ARL6IP1 ATCAY ABHD16A ACTN4 AES ALG3 ANO2 ARAP3 ARL6IP5 ATE1 ABHD3 ACTR10 AFAP1 ALK ANO3 ARC ARL8A ATF2 ABHD5 ACTR1A AFAP1L1 ALKBH4 ANO4 ARCN1 ARMC1 ATF7 ABHD6 ACTR1B AFF1 ALKBH5 ANO6 ARF1 ARMC12 ATG13 ABHD8 ACTR2 AFF3 ALOX12B ANO8 ARF6 ARMC2 ATG14 ABI1 ACTR3B AFF4 ALOX15 ANO9 ARFGAP1 ARMC6 ATG16L2 ABI2 ACTR6 AFG3L2 ALOX5AP ANP32A ARFGEF1 ARMC8 ATG4D ABL1 ACTR8 AGAP1 ALPI ANP32B ARFGEF2 ARMCX1 ATG7 ABLIM1 ACVR1 AGAP2 ALS2 ANTXR1 ARFIP2 ARMCX2 ATG9A ABLIM2 ACVR1B AGFG1 ALS2CR12 ANXA1 ARFRP1 ARMCX5 ATHL1 ABP1 ACVR2A AGGF1 ALX3 ANXA7 ARG1 ARNT ATL1 ABRA ACVR2B AGPAT3 ALX4 AOC2 ARGLU1 ARNT2 ATL2 ABT1 ACY3 AGPAT4 AMBP AOX1 ARHGAP1 ARNTL ATL3 ACAA2 ADA AGPAT5 AMD1 AP1B1 ARHGAP11A ARPC1A ATMIN ACACA ADAD1 AGPAT6 AMDHD2 AP1G1 ARHGAP12 ARPC1B ATOH1 
ACACB ADAM10 AGPAT9 AMIGO2 AP1G2 ARHGAP15 ARPC2 ATP11A

ACAD10 ADAM15 AGPS AMMECR1 AP1M1 ARHGAP17 ARPC4-TTLL3 ATP11B

ACAD8 ADAM20 AGR2 AMN1 AP1M2 ARHGAP18 ARPC5 ATP11C ACAD9 ADAM21 AGR3 AMOTL1 AP1S1 ARHGAP20 ARPC5L ATP12A ACADL ADAM22 AGRN AMPD2 AP2A1 ARHGAP21 ARPP21 ATP13A1 ACADM ADAM30 AGTRAP AMPD3 AP2A2 ARHGAP22 ARR3 ATP13A4 ATP1A2 B3GNT1 BEX1 BTF3 C1orf172 C8orf34 CAMK1D CCDC115 ATP1A3 B3GNT2 BFSP2 BTG2 C1orf189 C8orf40 CAMK1G CCDC121 ATP1B1 B4GALT1 BGN BTG3 C1orf226 C8orf46 CAMK2A CCDC124 ATP1B2 B4GALT2 BHLHE40 BTG4 C1orf51 C8orf76 CAMK2B CCDC132 ATP1B3 B4GALT3 BICD1 BTK C1orf52 C9orf117 CAMK2G CCDC134 ATP2A1 B4GALT7 BICD2 BTN3A3 C1orf61 C9orf152 CAMK4 CCDC138 ATP2A2 B9D1 BID BTNL9 C1orf65 C9orf156 CAMKK1 CCDC14 ATP2A3 B9D2 BIN1 BTRC C1orf74 C9orf171 CAMKK2 CCDC147 ATP2B2 BABAM1 BIN3 BUB1 C1QA C9orf174 CAMKMT CCDC149 ATP2B3 BACE1 BIRC2 BUB1B C1QC C9orf3 CAMKV CCDC15 ATP2B4 BACE2 BIRC3 BUB3 C1QTNF1 C9orf41 CAMLG CCDC155 ATP2C1 BACH1 BIRC6 BZW1 C1QTNF2 C9orf72 CAMSAP1 CCDC167 ATP2C2 BACH2 BIVM BZW2 C1QTNF3 C9orf78 CAMSAP2 CCDC171 ATP5A1 BAD BLK C10orf107 C1QTNF7 C9orf89 CAMTA1 CCDC172 ATP5C1 BAG2 BLM C10orf116 C1RL C9orf9 CANX CCDC3 ATP5D BAG5 BLMH C10orf118 C2 CA10 CAP1 CCDC36 ATP5F1 BAG6 BLNK C10orf12 C20orf111 CA11 CAPN1 CCDC41 ATP5G3 BAI2 BLOC1S1 C10orf137 C20orf194 CA12 CAPN10 CCDC43 ATP5H BAI3 BMF C10orf2 C20orf195 CA14 CAPN12 CCDC47 ATP5L BAIAP2 BMI1 C10orf32 C20orf20 CA2 CAPN13 CCDC56 ATP6V0A1 BAIAP2L1 BMP1 C10orf57 C20orf24 CA5B CAPN3 CCDC58 ATP6V0A2 BAIAP2L2 BMP2 C10orf76 C20orf26 CA7 CAPN5 CCDC6 ATP6V0A4 BAIAP3 BMP6 C10orf88 C20orf27 CAB39 CAPN7 CCDC64 ATP6V0B BAK1 BMP7 C11orf30 C20orf43 CAB39L CAPNS1 CCDC75 ATP6V0C BAMBI BMPR1A C11orf41 C20orf96 CABIN1 CAPRIN1 CCDC80

ATP6V0D1 BANP BMPR1B C11orf49 C21orf59 CABLES2 CAPRIN2 CCDC84

ATP6V1A BARHL1 BMX C11orf53 C21orf62 CABP7 CAPSL CCDC88A ATP6V1B1 BARHL2 BNC1 C11orf57 C22orf13 CACFD1 CAPZB CCDC89 ATP6V1B2 BARX2 BNIP1 C11orf63 C22orf28 CACHD1 CARD11 CCDC93 ATP6V1C1 BAX BNIP2 C11orf70 C22orf32 CACNA1A CARD9 CCDC94 ATP6V1H BAZ1A BNIP3L C11orf85 C2CD2L CACNA1C CARHSP1 CCDC96

ATP7B BAZ1B BOC C12orf29 C2orf18 CACNA1D CARS CCDC97 ATP8A1 BAZ2A BOD1L1 C12orf4 C2orf29 CACNA1E CASC1 CCKAR 
ATP8B2 BAZ2B BPGM C12orf44 C2orf47 CACNA1F CASC3 CCKBR ATP8B3 BBOX1 BPIFA1 C12orf56 C2orf56 CACNA1G CASD1 CCL2 ATP9A BBS1 BPIFB2 C12orf57 C2orf62 CACNA1H CASK CCL21 ATPAF1 BBS10 BPTF C12orf76 C2orf65 CACNA1I CASKIN2 CCL5 ATPAF2 BBS2 BRAF C13orf33 C2orf78 CACNA1S CASP10 CCL7 ATR BBS7 BRAP C14orf101 C3 CACNA2D1 CASP14 CCM2 ATRIP BCAS3 BRD1 C14orf126 C3AR1 CACNA2D2 CASP2 CCNA1 ATRNL1 BCCIP BRD3 C14orf166 C3orf22 CACNA2D3 CASP3 CCNB1 ATXN1 BCKDHB BRD4 C14orf166B C3orf23 CACNB1 CASQ2 CCNB1IP1 ATXN2L BCKDK BRD9 C14orf43 C3orf37 CACNB2 CASR CCNB3 ATXN7L2 BCL2 BRF1 C14orf79 C3orf38 CACNB3 CASS4 CCNC ATXN7L3 BCL2L1 BRF2 C14orf93 C3orf45 CACNG1 CASZ1 CCND1 AUH BCL2L11 BRI3BP C15orf26 C3orf58 CACNG2 CAT CCND2 AURKAIP1 BCL2L2-PABPN1 BRIP1 C15orf38 C5 CACNG3 CATSPER1 CCNDBP1

AURKB BCL6 BRMS1 C15orf38-AP3S2 C5AR1 CACNG4 CATSPERB CCNE1

AURKC BCL6B BROX C15orf44 C5orf25 CACNG5 CATSPERG CCNF AUTS2 BCL7B BRPF1 C15orf52 C5orf28 CACNG7 CAV1 CCNJ AVIL BCL7C BRS3 C16orf5 C5orf30 CACTIN CBFA2T2 CCNK AVL9 BCL9 BRSK1 C16orf62 C5orf34 CACUL1 CBLB CCNL1 AVPR1A BCL9L BRSK2 C16orf7 C5orf42 CAD CBLL1 CCNT2 AWAT1 BCOR BRWD1 C16orf86 C6orf106 CADM1 CBLN2 CCNY AXIN1 BCORL1 BRWD3 C16orf93 C6orf130 CADM2 CBLN3 CCNYL1 AZGP1 BCR BSCL2 C17orf49 C6orf203 CADM3 CBS CCP110 AZI2 BCS1L BSDC1 C17orf62 C6orf222 CADPS CBX2 CCR2 AZIN1 BECN1 BSN C18orf1 C6orf62 CADPS2 CBX7 CCR4 B3GALNT1 BEGAIN BSND C18orf54 C7 CALB1 CBY1 CCR6 B3GALNT2 BEND5 BST2 C18orf8 C7orf25 CALB2 CCBL1 CCR7 B3GALT1 BEND6 BTAF1 C19orf10 C7orf26 CALCB CCDC101 CCS B3GALT2 BEND7 BTBD1 C19orf54 C7orf43 CALHM1 CCDC103 CCT2 B3GALT4 BEST1 BTBD10 C19orf66 C7orf53 CALHM3 CCDC106 CCT3 B3GALT5 BEST4 BTBD11 C19orf70 C7orf59 CALM3 CCDC108 CCT5 B3GAT1 BET1L BTBD2 C1orf112 C7orf60 CALR CCDC112 CCT6A B3GAT3 BET3L BTBD6 C1orf159 C7orf61 CAMK1 CCDC113 CCT7 CCT8 CDH23 CFL1 CHURC1-FNTB CMTM1 COPS2 CREBZF CTNND1 CCZ1 CDH3 CFTR CIAO1 CMTM3 COPS3 CREM CTNND2 CD14 CDH4 CGA CIB1 CMTM7 COPS4 CRHBP CTNS CD151 CDH6 CH25H CIB2 CNDP2 COPS5 CRHR1 CTPS1 CD160 CDH7 CHAC2 CIB3 CNFN COPS6 CRIP3 CTPS2 CD163 CDH8 CHAMP1 CIC CNGA3 COPS7A CRIPT CTSA CD164 CDH9 CHCHD2 CIDEB CNIH2 COPS7B CRISP1 CTSC CD19 CDIPT CHCHD7 CIITA CNIH3 COPZ1 CRISPLD1 CTSD CD1C CDK1 CHD1 CILP CNKSR2 COQ10A CRK CTSK 
CD22 CDK14 CHD2 CIR1 CNN1 COQ5 CRKL CTSL2 CD247 CDK17 CHD3 CIRBP CNN2 COQ7 CRLF1 CTSW CD28 CDK19 CHD4 CIRH1A CNOT1 CORIN CRLF3 CTTN CD2AP CDK2 CHD5 CISD2 CNOT10 CORO1A CRMP1 CTTNBP2 CD300A CDK20 CHD7 CISH CNOT2 CORO1B CROT CTTNBP2NL CD300LG CDK3 CHD8 CIT CNOT3 CORO1C CRP CUEDC1 CD36 CDK4 CHD9 CITED2 CNOT6 CORO2A CRTAC1 CUL1 CD37 CDK5R1 CHDH CIZ1 CNOT6L CORO2B CRTC1 CUL2 CD38 CDK5RAP1 CHEK1 CKAP4 CNOT7 CORO6 CRTC2 CUL3 CD3D CDK5RAP2 CHEK2 CKAP5 CNOT8 CORT CRTC3 CUL4A CD47 CDK8 CHFR CKB CNP COTL1 CRY1 CUL4B CD53 CDK9 CHI3L1 CKLF CNRIP1 COX15 CRY2 CUL5 CD59 CDKAL1 CHIC2 CKM CNTFR COX16 CRYAA CUL7 CD63 CDKL2 CHID1 CKMT2 CNTN2 COX18 CRYAB CUL9 CD70 CDKL5 CHKA CLASRP CNTN4 COX4I1 CRYBA2 CUTC CD74 CDKN2AIP CHKB CLCA4 CNTN5 COX5A CRYBB1 CUX1 CD79B CDKN3 CHL1 CLCN3 CNTNAP1 COX6A1 CRYGS CUX2 CD81 CDO1 CHML CLCN6 CNTNAP2 COX7A2L CRYL1 CWC25 CD82 CDR2L CHMP1B CLCN7 CNTNAP4 CPA2 CSAD CWC27 CD9 CDS2 CHMP2A CLDN1 CNTRL CPA4 CSDC2 CX3CL1 CD93 CDYL CHMP2B CLDN10 COA1 CPB2 CSGALNACT1 CXCL17 CD96 CDYL2 CHMP3 CLDN11 COASY CPEB1 CSGALNACT2 CXCR3 CD99L2 CEACAM16 CHMP4B CLDN14 COBRA1 CPEB3 CSH2 CXCR4 CDA CEBPE CHMP5 CLDN15 COG2 CPEB4 CSK CXCR5 CDADC1 CECR1 CHMP6 CLDN16 COG3 CPLX1 CSMD1 CXCR6 CDAN1 CELA2A CHMP7 CLDN25 COG7 CPLX4 CSMD2 CXorf21 CDC123 CELA3A CHN1 CLDN4 COG8 CPN1 CSMD3 CXorf23 CDC14A CELF1 CHN2 CLDN9 COIL CPNE4 CSNK1A1 CXorf26 CDC14B CELF2 CHODL CLDND1 COL11A1 CPNE6 CSNK1D CXorf57 CDC16 CELF3 CHP1 CLEC2L COL12A1 CPNE7 CSNK1E CXXC5 CDC20 CELF4 CHPF CLIC1 COL13A1 CPNE8 CSNK1G1 CYB561 CDC25A CELF5 CHRD CLIC4 COL17A1 CPS1 CSNK1G2 CYB561D2 CDC27 CELSR1 CHRM1 CLINT1 COL19A1 CPSF1 CSNK1G3 CYB5B CDC34 CELSR2 CHRM3 CLIP2 COL1A1 CPSF2 CSNK2A1 CYB5D2 CDC37 CELSR3 CHRNA1 CLIP3 COL20A1 CPSF3 CSNK2B CYB5R1 CDC37L1 CEND1 CHRNA2 CLIP4 COL25A1 CPSF3L CSPP1 CYBASC3 CDC40 CENPB CHRNA4 CLK1 COL2A1 CPSF4 CSRNP1 CYC1 CDC42 CENPE CHRNA5 CLK2 COL3A1 CPSF4L CSRNP2 CYFIP1 CDC42BPA CENPM CHRNA6 CLK3 COL4A1 CPSF6 CSRNP3 CYGB CDC42BPB CEP104 CHRNB1 CLMN COL4A2 CPSF7 CSRP2 CYHR1 CDC42BPG CEP112 CHRNB2 CLN3 COL4A3BP CPT1A CSRP3 CYP11B1 CDC42EP1 CEP128 CHRNB3 CLN6 COL5A1 CPT1B CST11 CYP17A1 CDC42EP3 CEP170 CHRND CLN8 COL5A2 CPT1C CSTF1 CYP19A1 CDC42EP4 CEP250 CHRNE CLNS1A COL6A1 CPXM2 CSTF2T CYP1A2 
CDC42SE2 CEP63 CHRNG CLOCK COL6A3 CPZ CSTF3 CYP26A1 CDC45 CEP72 CHST1 CLPP COL6A6 CRABP1 CTAGE1 CYP27A1 CDC5L CEP78 CHST11 CLPTM1L COL7A1 CRABP2 CTBP1 CYP27B1 CDC7 CEP85 CHST14 CLPX COL8A1 CRAMP1L CTC1 CYP2E1 CDC73 CEP95 CHST15 CLRN1 COL9A1 CRB1 CTCF CYP2J2 CDCA7 CEP97 CHST3 CLRN2 COLEC10 CRB3 CTDP1 CYP2R1 CDCA8 CERS1 CHST6 CLSTN2 COLEC11 CRBN CTDSP2 CYP2U1 CDCP2 CERS2 CHST8 CLSTN3 COLQ CREB3 CTGF CYP3A4 CDH10 CES2 CHSY1 CLTB COMMD1 CREB3L2 CTHRC1 CYP3A5 CDH12 CES4A CHSY3 CLTC COMMD4 CREB3L3 CTNNA1 CYP3A7 CDH17 CETN2 CHTF8 CLVS1 COMMD7 CREB5 CTNNA2 CYP46A1 CDH18 CETP CHTOP CMAS COMP CREBBP CTNNB1 CYP4F11 CDH20 CFDP1 CHUK CMIP COMT CREBRF CTNNBL1 CYP4F22 CYP4X1 DDR1 DHRS11 DNAJB12 DRAP1 ECHDC1 EIF3E ENTPD4 CYP51A1 DDR2 DHRS13 DNAJB14 DRD1 ECHS1 EIF3F EP300 CYP7B1 DDX1 DHRS3 DNAJB2 DRD2 ECM1 EIF3G EP400 CYP8B1 DDX10 DHX15 DNAJB4 DRD3 ECSIT EIF3H EPAS1 CYR61 DDX17 DHX30 DNAJB5 DRG1 ECT2 EIF3I EPB41 CYSLTR1 DDX19A DHX32 DNAJB6 DROSHA EDAR EIF3L EPB41L1 CYSTM1 DDX21 DHX33 DNAJB8 DRP2 EDC3 EIF3M EPB41L4B CYTH1 DDX25 DHX35 DNAJC1 DSC2 EDC4 EIF4A1 EPB42 CYTH2 DDX26B DHX40 DNAJC11 DSCAM EDEM2 EIF4A2 EPB49 CYTH3 DDX27 DHX58 DNAJC14 DSCAML1 EDEM3 EIF4A3 EPC1 CYTIP DDX39A DIABLO DNAJC16 DSCR3 EDIL3 EIF4B EPC2 D2HGDH DDX39B DIAPH1 DNAJC18 DSE EDN1 EIF4E2 EPG5 D4S234E DDX3X DICER1 DNAJC19 DSEL EDN2 EIF4E3 EPGN DAAM1 DDX4 DIDO1 DNAJC2 DSG3 EDN3 EIF4EBP2 EPHA1 DAB2IP DDX49 DIMT1 DNAJC21 DSN1 EDNRA EIF4G1 EPHA2 DACH2 DDX5 DIO1 DNAJC22 DSP EEA1 EIF5 EPHA3 DAD1 DDX50 DIO3 DNAJC27 DST EED EIF5A EPHA4 DAG1 DDX55 DIP2A DNAJC3 DTD1 EEF1A1 EIF5B EPHA5 DAGLA DDX56 DIP2B DNAJC5 DTNA EEF1A2 ELAC2 EPHA6 DAK DDX6 DIP2C DNAJC6 DTNB EEF1E1 ELAVL1 EPHA7 DALRD3 DDX60 DIRAS1 DNAJC7 DTWD2 EEF1G ELAVL2 EPHB1 DAO DEAF1 DIRAS2 DNAJC8 DTX1 EEF2 ELAVL3 EPHB2 DAP3 DECR2 DIRAS3 DNAL4 DTX2 EEF2K ELAVL4 EPHB3 DAPK1 DEDD DIRC2 DNASE1L2 DTX3 EEFSEC ELF4 EPHB4 DAPK3 DEDD2 DIS3L DNM1 DTX3L EEPD1 ELF5 EPHB6 DAPP1 DEFB1 DIS3L2 DNM2 DTX4 EFCAB1 ELFN2 EPHX3 DAXX DEFB110 DKK4 DNMBP DTYMK EFCAB9 ELK3 EPHX4 DAZAP1 DEFB113 DLC1 DNMT1 DUOX1 EFEMP1 ELK4 EPN1 DBN1 DEFB121 DLD DNMT3B DUOXA2 EFEMP2 ELL EPN2 DBNDD1 DEFB134 DLG2 DNMT3L DUS1L EFHA2 ELMO1 EPRS DBR1 DEFB136 DLG3 DNTT DUS2L EFHD1 ELMO2 EPS15L1 
DCAF10 DEGS1 DLG4 DNTTIP2 DUS3L EFHD2 ELMOD1 EPS8

DCAF11 DEK DLG5 DOC2A DUSP10 EFNA4 ELN EPS8L2

DCAF12 DENND1A DLGAP1 DOCK11 DUSP12 EFNA5 ELOVL2 EPT1

DCAF12L1 DENND1B DLGAP2 DOCK3 DUSP14 EFNB2 ELOVL6 ERAS

DCAF12L2 DENND2A DLGAP3 DOCK4 DUSP16 EFR3A EMC10 ERBB2

DCAF15 DENND2D DLGAP4 DOCK5 DUSP18 EFR3B EMC2 ERBB3

DCAF17 DENND3 DLGAP5 DOCK7 DUSP26 EGFR EMC8 ERBB4

DCAF6 DENND4B DLK2 DOCK8 DUSP3 EGLN2 EMD ERC1

DCAF8 DENND5A DLL4 DOCK9 DUSP4 EGLN3 EMILIN1 ERC2

DCBLD1 DENND5B DLST DOK1 DUSP7 EGR1 EMILIN2 ERCC1

DCC DEPDC1 DLX1 DOK2 DVL2 EGR2 EMILIN3 ERCC2

DCDC1 DEPDC1B DLX2 DOK3 DYM EGR3 EML1 ERCC3

DCHS1 DEPDC4 DLX3 DOK4 DYNC1H1 EHBP1 EML3 ERCC4

DCLK1 DEPDC7 DLX5 DOK5 DYNC1I1 EHD1 EML4 ERCC6L

DCLK2 DERA DLX6 DOLK DYNC1I2 EHD4 EML5 ERCC8

DCP2 DERL1 DMAP1 DOLPP1 DYNC1LI2 EHF EML6 ERF

DCPS DERL2 DMBX1 DOPEY2 DYNC2H1 EHMT1 EMP2 ERG

DCT DES DMC1 DOT1L DYNLT1 EHMT2 EMX2 ERGIC1

DCTD DESI1 DMD DPEP1 DYRK1A EID1 ENAH ERGIC2

DCTN1 DFFB DMPK DPEP2 DYRK1B EIF2A ENAM ERGIC3

DCTN4 DFNA5 DMRT1 DPF1 DYRK2 EIF2AK2 ENC1 ERI1

DCTN5 DFNB59 DMRTC2 DPF2 DYSF EIF2AK4 ENDOU ERI3

DCTN6 DGAT1 DMTF1 DPF3 DZIP1 EIF2B1 ENG ERLEC1

DCUN1D1 DGAT2L6 DMWD DPH2 E2F4 EIF2B2 ENGASE ERLIN1

DCUN1D3 DGKD DMXL1 DPP8 E2F8 EIF2B3 ENO1 ERLIN2

DCUN1D4 DGKE DMXL2 DPP9 E4F1 EIF2B4 ENO2 ERN1

DCX DGKG DNAH1 DPY19L1 EBAG9 EIF2B5 ENO3 ERN2

DDAH1 DGKH DNAH2 DPY19L3 EBF1 EIF2C1 ENOPH1 ERO1L

DDAH2 DGKQ DNAH3 DPYSL2 EBF2 EIF2C2 ENOX1 ERP29

DDHD1 DGUOK DNAH5 DPYSL3 EBF3 EIF2C4 ENOX2 ERP44

DDHD2 DHCR24 DNAI1 DPYSL4 EBI3 EIF2D ENPP1 ERVFRD-1

DDI2 DHCR7 DNAJA1 DPYSL5 EBP EIF2S1 ENPP6 ESCO1

DDIT4 DHDDS DNAJA2 DQX1 EBPL EIF3A ENTHD1 ESF1

DDIT4L DHPS DNAJA3 DRAM1 ECE1 EIF3B ENTPD1 ESRP1

DDOST DHRS1 DNAJB11 DRAM2 ECE2 EIF3D ENTPD3 ESRP2

ESRRA FAM117A FAM73A FBXW11 FLOT1 FTL GAPDH GIPC3

ESRRG FAM118B FAM76A FBXW2 FLOT2 FTO GAPDHS GIT1

ESYT1 FAM120A FAM78A FBXW4 FLRT1 FTSJ2 GAPVD1 GIT2

ESYT2 FAM120C FAM78B FBXW5 FLRT3 FUBP1 GARS GJA1

ETF1 FAM122A FAM81A FBXW7 FLT1 FUBP3 GAS6 GJA10

ETFDH FAM123B FAM82A2 FBXW8 FLT3LG FURIN GAS7 GJA5

ETHE1 FAM125B FAM82B FCF1 FLYWCH1 FUT1 GATA1 GJA8

ETS1 FAM126B FAM83B FCGR3B FMOD FUT8 GATA2 GJC1

ETV1 FAM127C FAM83C FCGRT FMR1NB FXR1 GATA3 GJD2 
ETV3 FAM129B FAM84A FCHSD2 FN1 FXR2 GATAD2B GK ETV5 FAM131A FAM91A1 FCN1 FN3K FYB GATC GK2 ETV6 FAM131B FAM96A FCRL2 FNBP1 FYN GATM GK5 EVC2 FAM134C FAM98B FEM1B FNBP1L FZD3 GATS GKAP1 EVI5L FAM135A FAM9C FEM1C FNDC3A FZD4 GATSL3 GKN1 EVL FAM135B FANCA FEN1 FNDC3B FZD5 GBA2 GLB1L EWSR1 FAM13A FANCB FER FNDC4 FZD7 GBF1 GLB1L2 EXD1 FAM160B1 FANCE FER1L6 FNDC5 FZD8 GBP1 GLB1L3 EXOC1 FAM160B2 FANCI FERD3L FNDC8 FZD9 GBX2 GLCCI1 EXOC3 FAM166A FANCL FERMT1 FNIP1 FZR1 GCC2 GLDC EXOC3L2 FAM168A FAR1 FERMT2 FNIP2 G3BP1 GCDH GLE1 EXOC4 FAM168B FAR2 FERMT3 FNTA G3BP2 GCET2 GLG1 EXOC6B FAM169A FARS2 FEZ2 FNTB G6PC GCFC1 GLI1 EXOC8 FAM171A1 FARSB FEZF2 FOCAD G6PD GCH1 GLI2 EXOG FAM172A FASLG FGA FOPNL GABARAPL2 GCHFR GLI3 EXOSC1 FAM175B FASN FGD1 FOSB GABBR1 GCK GLIPR1 EXOSC10 FAM184A FASTK FGD4 FOSL2 GABBR2 GCKR GLIPR1L1 EXOSC2 FAM189A2 FASTKD1 FGF11 FOXA3 GABPA GCLC GLIPR2 EXOSC4 FAM18A FASTKD5 FGF12 FOXB1 GABRA1 GCLM GLIS2 EXOSC5 FAM196A FAT1 FGF13 FOXG1 GABRA2 GCM1 GLO1 EXOSC9 FAM198B FAT4 FGF17 FOXI1 GABRA3 GCN1L1 GLOD4 EXT2 FAM19A1 FATE1 FGF18 FOXJ2 GABRA5 GCNT2 GLRA1 EXTL2 FAM204A FAXC FGF21 FOXK2 GABRB1 GDAP1 GLRA2 EXTL3 FAM208A FBF1 FGF8 FOXN1 GABRB2 GDAP1L1 GLRA3 EYA1 FAM20A FBLN1 FGFR1 FOXN2 GABRB3 GDAP2 GLRX EYA3 FAM20B FBLN5 FGFR1OP2 FOXN3 GABRD GDE1 GLRX3 EYA4 FAM210A FBLN7 FGFR2 FOXN4 GABRG1 GDF11 GLS2 EZH1 FAM212B FBN1 FGFR3 FOXO1 GABRG2 GDF2 GLT25D1 EZH2 FAM214B FBN2 FGFRL1 FOXO3 GABRG3 GDF5 GLT25D2 EZR FAM217B FBXL12 FGL2 FOXO4 GABRQ GDI1 GLT8D1 F10 FAM222B FBXL14 FGR FOXP1 GAD1 GDI2 GLT8D2 F11R FAM227B FBXL16 FH FOXP2 GAD2 GDPD1 GLTP F2 FAM24A FBXL18 FHIT FOXP3 GAK GDPD5 GLUL F2R FAM26D FBXL19 FHOD1 FOXRED1 GALE GEMIN6 GLYR1 F2RL1 FAM35A FBXL2 FHOD3 FPGS GALK1 GEMIN7 GMCL1 F8 FAM3A FBXL20 FIBIN FPGT-TNNI3K GALK2 GET4 GMDS FA2H FAM3C FBXL3 FIBP FPR2 GALM GFOD1 GMEB1 FAAH FAM40A FBXL6 FICD FPR3 GALNT1 GFOD2 GMEB2 FAAH2 FAM40B FBXL7 FIG4 FREM1 GALNT10 GFPT1 GMPPA FABP7 FAM46B FBXO10 FIGF FREM2 GALNT11 GFPT2 GMPPB FADS1 FAM46C FBXO11 FILIP1 FRG1 GALNT12 GFRA1 GMPR FADS2 FAM48A FBXO15 FILIP1L FRK GALNT13 GFRA3 GMPR2 FADS3 FAM49A FBXO18 FIP1L1 FRMD4A GALNT14 GGA3 GMPS FADS6 FAM49B FBXO21 FITM1 FRMD6 GALNT2 GGCX GNA11 
FAF1 FAM53B FBXO24 FKBP10 FRMD8 GALNT3 GGPS1 GNA12 FAF2 FAM53C FBXO25 FKBP11 FRMPD2 GALNT5 GGT1 GNA14 FAH FAM54B FBXO3 FKBP14 FRMPD4 GALNT6 GGT5 GNA15 FAHD1 FAM57A FBXO30 FKBP1B FRRS1 GALNT9 GGT7 GNAI1 FAHD2A FAM57B FBXO31 FKBP3 FRS2 GALNTL1 GHITM GNAI2 FAIM2 FAM59A FBXO33 FKBP7 FRY GALNTL2 GHRHR GNAI3 FAM102A FAM63B FBXO38 FKBP8 FRYL GALNTL4 GHSR GNAL FAM102B FAM64A FBXO40 FLCN FSD1 GALNTL6 GID8 GNAO1 FAM108B1 FAM65A FBXO42 FLI1 FSD2 GALT GIGYF1 GNAQ FAM110B FAM65C FBXO46 FLII FSHR GAMT GINS2 GNAT1 FAM111A FAM69B FBXO48 FLNA FSTL1 GAN GINS3 GNAZ FAM111B FAM70A FBXO8 FLNB FSTL4 GANAB GINS4 GNB1 FAM114A2 FAM71D FBXO9 FLNC FTCD GAP43 GIPC2 GNB2 GNB2L1 GPR18 GSDMD HCAR2 HIST1H2AD HNMT HSPA13 IGF2R GNB3 GPR182 GSK3A HCFC1 HIST1H2AE HNRNPA1 HSPA14 IGFALS GNB5 GPR20 GSK3B HCN1 HIST1H2AG HNRNPA2B1 HSPA1L IGFL2 GNG13 GPR21 GSPT2 HDAC1 HIST1H2AH HNRNPA3 HSPA4 IGJ GNL1 GPR22 GSR HDAC10 HIST1H2AI HNRNPAB HSPA5 IGLON5 GNL3L GPR37 GSS HDAC11 HIST1H2AJ HNRNPC HSPA8 IGSF1 GNPAT GPR37L1 GSTA1 HDAC2 HIST1H2AL HNRNPF HSPA9 IGSF21 GNPDA1 GPR39 GSTA4 HDAC4 HIST1H2AM HNRNPH1 HSPB3 IGSF3 GNPDA2 GPR4 GSTCD HDAC5 HIST1H2BA HNRNPH2 HSPB7 IGSF8 GNPNAT1 GPR45 GSTM2 HDAC8 HIST1H2BC HNRNPK HSPBP1 IHH GNPTAB GPR50 GSTP1 HDC HIST1H2BD HNRNPL HSPD1 IKBKB GNPTG GPR52 GTDC1 HDDC2 HIST1H2BF HNRNPM HSPG2 IKBKE GNRHR GPR56 GTDC2 HDGFRP2 HIST1H2BG HNRNPR HSPH1 IKZF3 GNS GPR61 GTF2A1 HDHD1 HIST1H2BH HNRNPU HTATIP2 IKZF4 GOLGA3 GPR63 GTF2F1 HDHD2 HIST1H2BJ HNRNPUL1 HTR1A IKZF5 GOLGB1 GPR65 GTF2F2 HDHD3 HIST1H2BK HNRNPUL2 HTR1B IL13RA1

GOLIM4 GPR68 GTF2H1 HDLBP HIST1H2BL HNRPDL HTR1D IL16 GOLPH3 GPR75 GTF2H3 HEATR2 HIST1H2BM HNRPLL HTR1E IL17A GOLT1A GPR77 GTF2H4 HEATR5B HIST1H2BN HOMER1 HTR1F IL17B GON4L GPR83 GTF2H5 HEBP1 HIST1H2BO HOMER3 HTR2C IL17C GOPC GPR85 GTF2IRD1 HECA HIST1H3B HOOK1 HTR5A IL17RD GORAB GPR97 GTF3C1 HECTD1 HIST1H3C HOOK3 HTR6 IL1B GORASP2 GPRASP1 GTF3C2 HECTD2 HIST1H3D HOXA11 HTRA3

IL1R2

GOT1 GPRC5A GTF3C3 HECTD3 HIST1H3E HOXA2 HTT IL1RAP GOT1L1 GPRC5B GTF3C4 HECW1 HIST1H3G HOXA5 HUNK IL1RAPL1 GP2 GPS1 GTPBP1 HECW2 HIST1H3H HOXA6 HUWE1 IL1RAPL2 GPAM GPS2 GTPBP2 HELT HIST1H3I HOXB13 HYAL2 IL1RL2 GPATCH1 GPSM1 GTSF1 HELZ HIST1H4A HOXB2 HYOU1 IL1RN GPATCH3 GPT2 GUCA1A HEMGN HIST1H4B HOXB3 IAPP IL21 
GPATCH8 GPX3 GUCY1B3 HEPACAM HIST1H4C HOXB5 IBTK IL22RA2 GPC2 GPX4 GUCY2D HEPHL1 HIST1H4D HOXC10 ICA1 IL23A GPC3 GPX7 GULP1 HERC1 HIST1H4E HOXC11 ICAM2 IL25 GPC4 GRAMD1B GUSB HERC2 HIST1H4H HOXC6 ICAM5 IL26 GPC5 GRB10 GXYLT1 HERC3 HIST1H4I HOXC8 ICK IL27RA GPCPD1 GRB14 GYG1 HERC5 HIST1H4J HOXD13 ID1 IL28RA GPD1L GRB2 GYLTL1B HERPUD1 HIST2H2AB HOXD4 ID2 IL2RA GPER GREB1 GYS1 HES1 HIST2H2AC HP IDE IL2RG GPHA2 GRHL1 GZF1 HEXA HIST3H2A HP1BP3 IDH2 IL31 GPI GRHL2 GZMA HEXIM1 HIST3H2BB HPCA IDH3B IL3RA GPKOW GRHPR GZMK HEY1 HIST3H3 HPCAL1 IDI1 IL4 GPM6A GRIA1 H1F0 HEY2 HIST4H4 HPCAL4 IFFO1 IL5 GPM6B GRIA2 H1FX HEYL HIVEP2 HPDL IFI27L2 IL6R GPN3 GRIA3 H2AFX HFE2 HIVEP3 HPGD IFI30 IL8 GPR107 GRID1 H2AFY HFM1 HK1 HPN IFI44 ILDR2 GPR116 GRID2 H2AFY2 HGD HK3 HPS3 IFIT1 ILK GPR123 GRIK1 H3F3B HGF HLCS HRAS IFITM1 ILKAP GPR124 GRIK3 H6PD HGS HLF HRASLS IFNA14 IMMP2L GPR125 GRIK4 HABP2 HIAT1 HM13 HRH2 IFNGR1 IMMT GPR126 GRIN2A HABP4 HIATL1 HMBOX1 HRH3 IFNW1 IMP4 GPR132 GRIN2B HACL1 HIBADH HMBS HRSP12 IFRD1 IMPAD1 GPR133 GRINA HADHA HIC2 HMCN1 HS3ST1 IFT122 IMPDH1 GPR137 GRIP1 HADHB HIF1A HMG20A HS3ST2 IFT140 IMPDH2 GPR137B GRIPAP1 HAGH HIF3A HMG20B HS6ST3 IFT172 IMPG2 GPR137C GRK6 HAL HINT1 HMGA1 HSD11B1 IFT20 INA GPR139 GRM1 HAO2 HINT2 HMGB1 HSD11B2 IFT43 ING1 GPR141 GRM2 HAPLN1 HIPK1 HMGCL HSD17B1 IFT52 ING2 GPR142 GRM3 HAPLN3 HIPK3 HMGCR HSD17B3 IFT57 ING3 GPR146 GRM4 HARBI1 HIPK4 HMGCS1 HSD3B7 IFT80 ING5 GPR149 GRM5 HARS2 HIRA HMGCS2 HSDL1 IFT81 INHBA GPR157 GRM6 HAS2 HIST1H1A HMGXB4 HSF1 IFT88 INHBE GPR158 GRM7 HAS3 HIST1H1C HMOX1 HSF2 IGDCC3 INO80B GPR161 GRM8 HAUS4 HIST1H1D HMOX2 HSF2BP IGF1R INO80D GPR17 GRN HAUS8 HIST1H1E HMP19 HSF4 IGF2 INO80E GPR171 GRPEL2 HAX1 HIST1H2AA HMSD HSP90AB1 IGF2BP1 INPP4A GPR173 GRSF1 HBP1 HIST1H2AB HNF1A HSP90B1 IGF2BP2 INPP4B GPR176 GRWD1 HCAR1 HIST1H2AC HNF1B HSPA12A IGF2BP3 INPP5A

INPP5B ITFG3 KATNA1 KCNV1 KIAA1217 KLHL24 LARP1 LIX1L INPP5F ITGA1 KATNAL1 KCNV2 KIAA1244 KLHL25 LARP4 LLGL1 INPP5J ITGA11 KATNB1 KCTD1 KIAA1279 KLHL26 LARP6 LMAN2 INPP5K ITGA2 KAZN KCTD10 KIAA1324 KLHL29 LARS LMAN2L INPPL1 ITGA2B KBTBD12 KCTD13 KIAA1324L KLHL3 LARS2 LMBR1 INS ITGA3 KBTBD2 KCTD14 KIAA1429 KLHL30 LAS1L LMBR1L 
INSIG1 ITGA4 KBTBD3 KCTD15 KIAA1432 KLHL32 LASP1 LMBRD1 INSIG2 ITGA5 KBTBD4 KCTD16 KIAA1467 KLHL34 LAT LMBRD2 INS-IGF2 ITGA8 KBTBD5 KCTD19 KIAA1468 KLHL36 LBR LMCD1 INSR ITGAD KBTBD6 KCTD2 KIAA1598 KLHL4 LBX1 LMF2 INSRR ITGAL KCMF1 KCTD21 KIAA1644 KLHL5 LCA5 LMLN INTS1 ITGAM KCNA1 KCTD3 KIAA1737 KLHL6 LCAT LMNB1 INTS10 ITGAV KCNA10 KCTD4 KIAA1841 KLHL7 LCE3E LMO1 INTS12 ITGB1 KCNA2 KCTD5 KIAA1967 KLHL8 LCLAT1 LMO4 INTS2 ITGB1BP1 KCNA4 KCTD6 KIAA2018 KLHL9 LCMT1 LMTK2 INTS3 ITGB3BP KCNA6 KCTD7 KIAA2022 KLK13 LCP2 LMX1A INTS4 ITGB4 KCNA7 KCTD8 KIDINS220 KLK5 LCT LMX1B INTS6 ITGB5 KCNAB1 KCTD9 KIF12 KLK7 LDB1 LNX1 INTS7 ITGB6 KCNAB2 KDELC1 KIF13A KLK8 LDB2 LNX2 INTS8 ITGB7 KCNAB3 KDELR1 KIF13B KLK9 LDB3 LOC81691 INTS9 ITGB8 KCNC1 KDELR2 KIF1A KLRC1 LDHA LONP1 INTU ITIH1 KCNC2 KDM1A KIF1B KLRK1 LDHAL6A LONP2 INVS ITIH4 KCNC4 KDM2A KIF1C KPNA1 LDHB LONRF2 IP6K1 ITK KCND1 KDM2B KIF20A KPNA3 LDHD LONRF3 IPO11 ITLN1 KCND2 KDM3A KIF21A KPNA4 LDLR LOXL2 IPO13 ITM2B KCND3 KDM3B KIF21B KPNA6 LDLRAD3 LOXL4 IPO4 ITM2C KCNF1 KDM4B KIF22 KPNB1 LDOC1L LPAR1 IPO5 ITPK1 KCNG1 KDM4C KIF23 KPTN LECT1 LPAR2 IPO7 ITPKB KCNG3 KDM5A KIF26B KRAS LEPR LPAR3 IPO8 ITPKC KCNH2 KDM5B KIF3A KRIT1 LEPREL1 LPCAT1 IPO9 ITPR1 KCNH3 KDM5C KIF3B KRR1 LEPREL4 LPCAT4 IPP ITPR2 KCNH4 KDM6A KIF3C KRT14 LGALS4 LPHN1 IQCD ITPR3 KCNH5 KDM6B KIF4A KRT17 LGALS9C LPHN2 IQCH ITPRIP KCNH6 KDM8 KIF5A KRT18 LGI1 LPHN3 IQGAP1 ITSN1 KCNH7 KDSR KIF7 KRT19 LGI2 LPIN1 IQGAP3 ITSN2 KCNH8 KEL KIFAP3 KRT222 LGR4 LPIN2 IQSEC1 IVD KCNIP2 KERA KIFC3 KRT38 LGR6 LPO IQSEC2 IVNS1ABP KCNIP4 KHDRBS1 KIN KRT6B LHFP LPPR1 IQSEC3 IZUMO1 KCNJ1 KHDRBS2 KIRREL KRT6C LHFPL2 LPPR2 IRAK3 IZUMO2 KCNJ13 KHDRBS3 KIRREL3 KRT73 LHFPL4 LPPR4 IRAK4 JAG1 KCNJ14 KHNYN KIT KRT85 LHFPL5 LPPR5 IREB2 JAG2 KCNJ2 KHSRP KITLG KRT9 LHX2 LRBA IRF2 JAK1 KCNJ4 KIAA0101 KLC1 KRTAP13-3 LHX4 LRCH3 IRF4 JAK2 KCNJ5 KIAA0146 KLC4 KRTAP19-5 LHX9 LRCH4 IRF5 JAKMIP1 KCNJ6 KIAA0182 KLF12 KRTAP19-7 LIAS LRFN2 IRF6 JAKMIP2 KCNJ9 KIAA0195 KLF15 KRTAP21-1 LIFR LRFN3 IRF7 JAKMIP3 KCNK1 KIAA0196 KLF3 KRTCAP2 LIG3 LRFN5 IRF8 JARID2 KCNK10 KIAA0232 KLF4 KRTDAP LIG4 LRIF1 IRF9 JAZF1 KCNK17 KIAA0240 KLF6 KY LILRA5 LRIG2 IRGC JDP2 KCNK2 KIAA0247 KLF7 KYNU LIM2 LRIG3 
IRS1 JHDM1D KCNK4 KIAA0284 KLHDC1 L1CAM LIMD1 LRIT1 IRX6 JKAMP KCNK6 KIAA0317 KLHDC10 L3MBTL3 LIMK1 LRIT2 ISG15 JMJD1C KCNK9 KIAA0319L KLHDC2 LACRT LIMS2 LRP1 ISG20 JMJD6 KCNMA1 KIAA0408 KLHDC3 LACTB2 LIN28A LRP10 ISL1 JPH3 KCNMB2 KIAA0430 KLHDC5 LAMA3 LIN54 LRP12 ISL2 JRKL KCNN2 KIAA0494 KLHDC8B LAMA4 LIN7A LRP1B ISLR JUN KCNN3 KIAA0513 KLHL1 LAMB2 LIN7B LRP2BP ISLR2 JUP KCNN4 KIAA0528 KLHL11 LAMB4 LIN7C LRP4 ISOC1 KAAG1 KCNQ1 KIAA0564 KLHL12 LAMC1 LIN9 LRP5 ISOC2 KALRN KCNQ2 KIAA0664 KLHL13 LAMP1 LINGO1 LRP6 IST1 KANSL1 KCNQ3 KIAA0895 KLHL15 LAMP3 LINGO2 LRP8 ISY1 KAT2B KCNQ4 KIAA0907 KLHL17 LAMTOR1 LINGO4 LRPPRC ISY1-RAB43 KAT5 KCNQ5 KIAA0913 KLHL18 LAP3 LIPC LRRC1 ISYNA1 KAT6A KCNT1 KIAA0947 KLHL20 LAPTM4A LIPH LRRC10 ITFG1 KAT6B KCNT2 KIAA1045 KLHL22 LAPTM4B LIPT1 LRRC15 ITFG2 KAT8 KCNU1 KIAA1109 KLHL23 LARGE LIX1 LRRC16A LRRC16B MAFB MAPKAPK3 MDFI MFAP5 MMP11 MRPS11 MYCN LRRC20 MAFG MAPKAPK5 MDH1 MFF MMP15 MRPS12 MYD88 LRRC23 MAG MAPKBP1 MDH1B MFGE8 MMP16 MRPS14 MYEF2 LRRC27 MAGEA3 MAPRE1 MDH2 MFHAS1 MMP17 MRPS15 MYF5 LRRC28 MAGEB4 MAPRE2 MDM2 MFI2 MMP19 MRPS18A MYH10 LRRC32 MAGEC2 MAPRE3 MDP1 MFN1 MMP2 MRPS23 MYH11 LRRC33 MAGED1 MARC2 ME1 MFN2 MMP21 MRPS35 MYH13 LRRC39 MAGED2 MARCH3 ME3 MFNG MMP24 MRPS5 MYH14 LRRC3B MAGEE2 MARCH4 MEAF6 MFRP MMP25 MRTO4 MYH2 LRRC4 MAGI1 MARCH5 MECP2 MFSD11 MMP3 MS4A1 MYH3 LRRC41 MAGI2 MARCH6 MED1 MFSD12 MMRN2 MS4A3 MYH6 LRRC42 MAGOHB MARCKSL1 MED10 MFSD2A MNAT1 MS4A8B MYH7

LRRC45 MAK MARCO MED12L MFSD5 MND1 MSANTD2 MYH7B LRRC47 MAK16 MARK1 MED13 MFSD7 MNT MSANTD3 MYH8 LRRC49 MAL MARK2 MED13L MGA MOB1B MSANTD4 MYH9 LRRC4B MALSU1 MARK3 MED14 MGAT3 MOB2 MSH2 MYL10 LRRC4C MALT1 MARS2 MED15 MGAT4C MOB3A MSH3 MYL2 LRRC52 MAMSTR MARVELD2 MED16 MGAT5 MOCS3 MSH6 MYL3 LRRC55 MAN1A1 MAS1 MED17 MGEA5 MON1A MSI1 MYL6B LRRC57 MAN1C1 MASP1 MED18 MGLL MON1B MSL1 MYL9 LRRC59 MAN2A1 MAST1 MED20 MGRN1 MON2 MSL2 MYLK LRRC7 MAN2A2 MAST2 MED22 MGST1 MORC2 MSL3 MYLK2 LRRC8A MAN2C1 MAST3 MED24 MGST3 MORC3 MSN MYLK3 LRRC8B MANBA MAST4 MED25 MIB1 MORC4 MSRB3 MYLPF LRRC8D MANBAL MAT1A MED26 MICAL1 MORF4L1 MST1 MYNN LRRFIP2 MANEAL MAT2A MED27 MICALL1 MORF4L2 MST1R MYO10 LRRK1 MAOA MAT2B MED28 MICU1 MORN1 MST4 MYO15A 
LRRN1 MAOB MATK MED29 MID1 MORN3 MTA3 MYO18A LRRN2 MAP1B MATN1 MED6 MID2 MORN4 MTAP MYO1B LRRN3 MAP2 MATR3 MED8 MIDN MOSPD2 MTCH1 MYO1D LRRTM2 MAP2K1 MAU2 MEF2A MIER1 MOV10 MTDH MYO1F LRRTM3 MAP2K2 MAX MEF2B MIER3 MOV10L1 MTF2 MYO1G LRRTM4 MAP2K4 MB MEF2D MIF4GD MOXD1 MTFP1 MYO1H LRSAM1 MAP2K5 MB21D2 MEGF10 MINK1 MPG MTFR1 MYO5A LRTM2 MAP2K6 MBD1 MEGF11 MINPP1 MPHOSPH8 MTG1 MYO5B LSM1 MAP2K7 MBD2 MEGF8 MIOX MPHOSPH9 MTHFD1L MYO6 LSM14B MAP3K1 MBD3 MEGF9 MIPOL1 MPI MTHFD2 MYO7A LSM3 MAP3K11 MBD5 MEI1 MIS12 MPL MTHFD2L MYO9A LSM4 MAP3K12 MBD6 MEIS1 MIS18A MPO MTIF2 MYO9B LSR MAP3K13 MBLAC2 MEIS2 MITD1 MPP1 MTM1 MYOC LTBP1 MAP3K2 MBNL1 MEIS3 MITF MPP3 MTMR1 MYOCD LTBP2 MAP3K5 MBNL2 MEN1 MKI67IP MPP4 MTMR3 MYOD1 LTK MAP3K7 MBNL3 MEPCE MKL1 MPP6 MTMR4 MYOF LUM MAP3K8 MBOAT7 MESDC2 MKL2 MPP7 MTMR8 MYOM2 LY6H MAP3K9 MBTPS1 MET MKLN1 MPPED1 MTMR9 MYOT LYG1 MAP4K1 MBTPS2 METAP1 MKNK2 MPPED2 MTO1 MYOZ1 LYN MAP4K3 MC3R METAP1D MKRN2 MPRIP MTOR MYOZ2 LYNX1 MAP4K4 MC4R METAP2 MLANA MPV17 MTPAP MYRIP LYPD6 MAP7D1 MCAM METRNL MLEC MPZ MTSS1 MYT1 LYPD6B MAPK1 MCC METTL1 MLF2 MPZL1 MTSS1L MYT1L LYPLA2 MAPK10 MCCC1 METTL13 MLL MRAP2 MUCL1 N4BP1 LYRM5 MAPK11 MCCC2 METTL14 MLL2 MRAS MUM1 N4BP3 LYST MAPK12 MCF2L METTL15 MLL3 MRFAP1 MURC NAA15 LZIC MAPK13 MCFD2 METTL16 MLL4 MRGPRF MUTED NAA16 LZTR1 MAPK14 MCHR2 METTL2B MLL5 MRPL10 MVK NAA20 LZTS2 MAPK1IP1L MCL1 METTL3 MLLT1 MRPL11 MVP NAA25 M6PR MAPK3 MCM3AP METTL5 MLLT10 MRPL14 MX1 NAA30 MAB21L1 MAPK4 MCM5 METTL6 MLLT3 MRPL19 MX2 NAA35 MAB21L2 MAPK6 MCM6 METTL9 MLLT4 MRPL2 MXD1 NAA40 MAB21L3 MAPK7 MCM7 MEX3A MLST8 MRPL22 MXD4 NAA60 MACF1 MAPK8 MCMBP MEX3B MMAA MRPL27 MXI1 NAB1 MAD1L1 MAPK8IP1 MCOLN3 MEX3C MMACHC MRPL3 MYADM NABP1

MAD2L1BP MAPK8IP3 MCRS1 MFAP2 MMD MRPL36 MYBL1 NABP2 MADD MAPK9 MCTP1 MFAP3 MMD2 MRPL42 MYBPC3 NACC1 MAEA MAPKAP1 MCTP2 MFAP3L MME MRPL44 MYBPH NADK MAF1 MAPKAPK2 MCU MFAP4 MMEL1 MRPL54 MYCL1 NADSYN1 NAE1 NDUFAF3 NGEF NPBWR2 NUAK1 OLA1 OTOP2 PARN NAGA NDUFAF5 NGFR NPDC1 NUAK2 OLFM1 OTUD5 PARP11 NAGK NDUFAF6 NGLY1 NPEPL1 NUB1 OLFM3 OTX1 PARP12 NAGLU NDUFB10 NHS NPL NUBP1 OLFML2A OTX2 PARP14 
NAGS NDUFB11 NICN1 NPLOC4 NUBP2 OLIG3 OVCA2 PARP16 NALCN NDUFB2 NIF3L1 NPM1 NUCB1 OLR1 OVOL1 PARP2 NAMPT NDUFB4 NIM1 NPR1 NUCB2 ONECUT1 OVOL2 PARP6 NANP NDUFB7 NIP7 NPRL2 NUCKS1 ONECUT2 OXCT1 PARP9 NAP1L1 NDUFB8 NIPA1 NPTN NUDC OPA1 OXGR1 PARS2 NAP1L2 NDUFS1 NIPAL3 NPTX1 NUDCD2 OPCML OXSR1 PARVA NAP1L4 NDUFS3 NIPBL NPY1R NUDT10 OPN1SW P2RX1 PATE2 NAP1L5 NDUFS4 NIPSNAP1 NPY2R NUDT11 OPN5 P2RX2 PATZ1 NAPB NDUFS5 NIPSNAP3A NPY5R NUDT14 OPRD1 P2RX6 PAX2 NAPG NDUFS6 NISCH NR0B1 NUDT17 OPRK1 P2RY1 PAX6 NARFL NDUFS7 NIT2 NR1D1 NUDT4 OPRL1 P2RY12 PAX8 NARG2 NDUFS8 NKAIN2 NR1H2 NUDT5 OPTN P2RY6 PAXIP1 NARS NDUFV1 NKAP NR1H3 NUF2 OR10J5 P2RY8 PBRM1 NARS2 NECAB1 NKD1 NR1I2 NUFIP1 OR13J1 P4HA1 PBX2 NASP NECAB2 NKIRAS1 NR1I3 NUMA1 OR1E1 P4HB PBX3 NAT10 NECAP1 NKIRAS2 NR2C1 NUMB OR1J4 P4HTM PBX4 NAT16 NEDD1 NKRF NR2C2 NUP107 OR1K1 PA2G4 PC NAV1 NEDD4L NKX2-5 NR2C2AP NUP155 OR2B3 PABPC1 PCBP1 NAV2 NEDD9 NLGN1 NR2E1 NUP160 OR2Z1 PABPC5 PCBP2 NAV3 NEGR1 NLGN3 NR2F1 NUP188 OR4A15 PACRG PCBP3 NBAS NEK1 NLGN4X NR2F2 NUP210 OR4C3 PACS2 PCBP4 NBEA NEK2 NLK NR3C1 NUP210L OR4D10 PACSIN1 PCDH10 NBEAL2 NEK4 NLN NR3C2 NUP214 OR4X2 PACSIN3 PCDH11X NCAM2 NEK6 NLRC4 NR4A1 NUP35 OR51T1 PADI1 PCDH17 NCAN NEK8 NLRP11 NR4A2 NUP37 OR56A1 PADI2 PCDH18 NCAPD2 NEK9 NLRP12 NR4A3 NUP43 OR56A3 PAF1 PCDH19 NCAPD3 NELF NLRP9 NR5A2 NUP50 OR56A4 PAFAH1B1 PCDH7 NCAPG NELL2 NMD3 NR6A1 NUP54 OR5C1 PAFAH1B2 PCDH9 NCAPH NEMF NME1 NRBP1 NUP62 OR5D16 PAFAH1B3 PCDHA13 NCAPH2 NEO1 NME1-NME2 NRCAM NUP93 OR5T1 PAFAH2 PCDHA3 NCBP1 NET1 NME2 NRF1 NUP98 OR6C75 PAG1 PCDHA6 NCBP2 NETO2 NME4 NRG3 NUPL1 OR7D2 PAH PCDHAC2 NCF4 NEU1 NME6 NRIP2 NXF1 OR9Q2 PAIP1 PCDHGA5 NCK2 NEU3 NME7 NRP1 NXF3 ORAI2 PAIP2B PCDHGA6 NCKAP1 NEURL4 NMI NRSN1 NXN ORAI3 PAK1 PCDHGB1 NCKAP1L NEUROD1 NMRAL1 NRXN1 NXPH1 ORC2 PAK2 PCDHGB4 NCKAP5L NEUROD6 NMT1 NRXN2 NXPH2 ORC5 PAK3 PCDHGB7 NCL NF1 NMT2 NRXN3 NXPH3 ORC6 PAK4 PCDHGC3 NCLN NF2 NNMT NSA2 NXPH4 ORMDL1 PAK7 PCDHGC4 NCOA1 NFAM1 NNT NSD1 NXT1 ORMDL2 PALM PCDHGC5 NCOA3 NFASC NOB1 NSDHL NYAP1 ORMDL3 PALM2 PCDP1 NCOA6 NFAT5 NOC4L NSFL1C O3FAR1 OS9 PAM PCED1B NCOA7 NFATC1 NOD1 NSMAF OAS3 OSBP PAMR1 PCF11 NCOR1 NFATC2 NODAL NSMCE2 OASL OSBP2 PAN2 PCGF2 
NCOR2 NFE2 NOL4 NSMCE4A OAT OSBPL10 PAN3 PCGF3 NCS1 NFE2L1 NOL6 NSUN3 OBSL1 OSBPL11 PANK2 PCGF5 NCSTN NFIA NOL9 NSUN6 OCIAD1 OSBPL1A PAPD4 PCGF6 NDC80 NFIB NOP56 NT5C1A OCRL OSBPL2 PAPD5 PCID2 NDE1 NFIC NOP58 NT5C2 ODC1 OSBPL3 PAPL PCIF1 NDFIP1 NFIX NOS2 NT5DC2 ODF2 OSBPL5 PAPOLA PCLO NDNF NFKB1 NOSIP NT5DC3 ODF2L OSBPL6 PAPOLG PCM1 NDRG1 NFKB2 NOTCH1 NT5M ODZ1 OSBPL7 PAPPA PCMTD2 NDRG3 NFKBIA NOTCH2 NTF3 ODZ3 OSBPL8 PAPPA2 PCNA NDRG4 NFKBIB NOTCH3 NTHL1 ODZ4 OSBPL9 PAPSS1 PCNX NDST1 NFKBID NOTUM NTMT1 OFD1 OSGEP PAPSS2 PCNXL3 NDST2 NFKBIZ NOVA1 NTN1 OGDH OSGIN2 PAQR3 PCOLCE NDUFA12 NFRKB NOVA2 NTN4 OGDHL OSM PAQR4 PCSK1 NDUFA4 NFS1 NOX3 NTNG1 OGFOD1 OSTalpha PAQR6 PCSK7 NDUFA4L2 NFX1 NOXRED1 NTNG2 OGFOD2 OSTF1 PAQR7 PCYOX1 NDUFA5 NFYA NPAS2 NTRK1 OGFRL1 OSTM1 PAQR8 PCYT1A NDUFA8 NFYC NPAS3 NTRK2 OGN OTOA PAQR9 PCYT1B NDUFAB1 NGDN NPAS4 NTRK3 OGT OTOF PARD6G PCYT2 PDAP1 PFKFB3 PIGN PLD5 POLK PPM1B PRKAR2A PSMA2 PDC PFKL PIGO PLEC POLL PPM1D PRKAR2B PSMA3 PDCD1 PFKM PIGR PLEK2 POLR1B PPM1E PRKCA PSMA4 PDCD11 PFKP PIGT PLEKHA1 POLR1C PPM1G PRKCB PSMA5 PDCD2L PFN1 PIGU PLEKHA3 POLR1D PPM1K PRKCG PSMA7 PDCD4 PGAM2 PIGV PLEKHA5 POLR2A PPME1 PRKCH PSMA8 PDCD6 PGAP1 PIK3C2A PLEKHA6 POLR2C PPOX PRKCI PSMB10 PDCD6IP PGAP2 PIK3C3 PLEKHA7 POLR2D PPP1CA PRKCQ PSMB4 PDCL PGBD2 PIK3CA PLEKHB1 POLR2E PPP1CB PRKCSH PSMB6 PDDC1 PGBD3 PIK3CB PLEKHB2 POLR2I PPP1R10 PRKD2 PSMC1 PDE12 PGBD4 PIK3CD PLEKHF1 POLR2L PPP1R12A PRKG1 PSMC2 PDE1A PGD PIK3CG PLEKHF2 POLR3A PPP1R12B PRKG2 PSMC3 PDE1B PGF PIK3R1 PLEKHG3 POLR3B PPP1R13B PRKRIR PSMC3IP PDE4B PGK1 PIK3R2 PLEKHG4B POLR3D PPP1R16A PRKX PSMC4 PDE4D PGM1 PIK3R3 PLEKHG6 POLR3E PPP1R16B PRL PSMC5 PDE6A PGM3 PIK3R5 PLEKHH2 POLR3F PPP1R27 PRLH PSMC6 PDE6B PGM5 PIKFYVE PLEKHM1 POLR3G PPP1R7 PRM2 PSMD1 PDE7A PGPEP1 PILRB PLEKHM2 POLR3GL PPP1R8 PRMT1 PSMD10 PDE7B PGS1 PIM2 PLEKHO1 POLR3H PPP2CA PRMT10 PSMD11 PDE8A PHACTR3 PINK1 PLIN3 POLR3K PPP2CB PRMT3 PSMD12 PDE8B PHACTR4 PIP4K2C PLK1 POM121 PPP2R1A PRMT5 PSMD2 PDE9A PHAX PIP5K1A PLK2 POMGNT1 PPP2R2A PRMT6 PSMD3 PDGFA PHB2 PIP5K1C PLK4 POMP PPP2R2B PRMT7 PSMD4 PDGFD PHC2 PIPOX PLLP POMT1 PPP2R2C PRMT8 PSMD5 PDGFRA PHC3 PISD PLOD1 POP1 PPP2R3C PROC PSMD6 PDGFRB PHF1 PITPNA PLRG1 POP5 PPP2R5A PROCA1 PSMD7 
PDHB PHF10 PITPNM2 PLS1 POPDC2 PPP2R5B PROKR1 PSMD8 PDIA3 PHF11 PITPNM3 PLS3 POR PPP2R5D PROKR2 PSME3 PDIA4 PHF13 PITX1 PLSCR5 PORCN PPP2R5E PROL1 PSPC1 PDIA6 PHF14 PITX2 PLTP POU2AF1 PPP3CA PROM1 PTBP1 PDIK1L PHF15 PIWIL1 PLVAP POU2F1 PPP3CB PROM2 PTBP2 PDK1 PHF16 PIWIL2 PLXNA1 POU2F2 PPP4C PROS1 PTBP3 PDK2 PHF17 PJA2 PLXNA2 POU4F2 PPP5C PROSC PTCD2 PDLIM1 PHF20 PKD2 PLXNA3 POU4F3 PPP6C PROSER1 PTCD3 PDLIM3 PHF20L1 PKD2L2 PLXNA4 POU5F1 PPT1 PROX1 PTCH1 PDLIM7 PHF21A PKDREJ PLXNB1 POU6F1 PPTC7 PROX2 PTCH2 PDP1 PHF23 PKM PLXNB2 POU6F2 PPWD1 PROZ PTCHD2 PDS5A PHF3 PKMYT1 PLXNB3 PPA1 PQLC1 PRPF19 PTCHD4 PDS5B PHGDH PKN3 PLXNC1 PPAN PQLC3 PRPF3 PTDSS1 PDSS2 PHIP PKNOX1 PLXND1 PPAN-P2RY11 PRC1 PRPF38A PTDSS2 PDXK PHKA1 PKNOX2 PMCH PPAP2A PRCC PRPF4 PTEN PDZD4 PHKA2 PLA2G12B PMFBP1 PPAP2B PRDM15 PRPF40B PTGDS PDZD7 PHKG1 PLA2G15 PMM1 PPAPDC1A PRDM16 PRPF4B PTGER2 PEAK1 PHKG2 PLA2G1B PMM2 PPAPDC3 PRDM2 PRPF6 PTGER4 PEF1 PHLDA2 PLA2G4A PMPCA PPARA PRDM4 PRPF8 PTGFRN PEG10 PHLDB1 PLA2G5 PMPCB PPARD PRDM5 PRPS2 PTGIS PELI1 PHLPP2 PLA2G6 PNCK PPARG PRDX1 PRPSAP1 PTGR2 PELI2 PHRF1 PLAC1 PNKD PPARGC1A PRDX2 PRPSAP2 PTGS2 PELO PHTF1 PLAG1 PNKP PPBP PRDX6 PRR14 PTH PEPD PHTF2 PLAGL1 PNLIP PPCDC PREB PRRC2B PTH2R PER1 PHYHIP PLAGL2 PNMA2 PPDPF PRELID1 PRRC2C PTK2 PER2 PHYHIPL PLAT PNN PPEF1 PREPL PRSS1 PTK2B PES1 PI4K2B PLBD2 PNOC PPFIA1 PREX2 PRSS16 PTK7 PEX1 PI4KA PLCB1 PNPLA4 PPFIA4 PRF1 PRSS22 PTOV1 PEX10 PI4KB PLCB2 PNPLA8 PPFIBP1 PRG2 PRSS3 PTP4A1 PEX11A PIAS1 PLCB3 PNPO PPFIBP2 PRIC285 PRSS35 PTP4A3 PEX11B PIAS2 PLCB4 POC1B PPHLN1 PRICKLE1 PRSS53 PTPDC1 PEX14 PIAS3 PLCD1 POFUT1 PPIB PRICKLE3 PRSS57 PTPN1 PEX19 PIAS4 PLCG1 POFUT2 PPIE PRIMA1 PRSS8 PTPN11 PEX3 PICALM PLCG2 POGZ PPIH PRKAA1 PRTG PTPN12 PEX5 PICK1 PLCL1 POLA1 PPIL2 PRKAA2 PRX PTPN14 PFAS PID1 PLCL2 POLA2 PPIL4 PRKACA PSAT1 PTPN18 PFDN2 PIDD PLCXD2 POLB PPIL6 PRKACB PSD PTPN23 PFDN5 PIGH PLCXD3 POLD1 PPIP5K2 PRKACG PSEN1 PTPN5 PFKFB1 PIGK PLCZ1 POLE PPL PRKAG2 PSKH1 PTPN6 PFKFB2 PIGM PLD3 POLE3 PPM1A PRKAR1A PSMA1 PTPN9 PTPRD RAB40C RAPH1 RBMS1 RGS12 RNF150 RPLP2 RUSC1 PTPRE RAB41 RARA RBMS2 RGS13 RNF152 RPN2 RUSC2 PTPRF RAB4A RARB RBMS3 RGS17 RNF165 RPP25L RUVBL1 PTPRG RAB4B RARG RBP2 RGS19 RNF166 RPRD1A RWDD1 
PTPRK RAB5A RARRES3 RBP4 RGS2 RNF167 RPRD1B RWDD2A PTPRM RAB5B RARS RBPJ RGS20 RNF169 RPRD2 RXFP1 PTPRN RAB7A RARS2 RC3H1 RGS3 RNF182 RPS10 RXFP2 PTPRO RAB8A RASA1 RC3H2 RGS4 RNF19A RPS11 RXFP3 PTPRS RAB8B RASA2 RCAN1 RGS6 RNF19B RPS14 RXRA PTPRU RAB9B RASA3 RCAN3 RGS7 RNF2 RPS16 RXRB PTPRZ1 RABAC1 RASAL2 RCBTB1 RHBDD2 RNF20 RPS19 RXRG PTRHD1 RABEP1 RASAL3 RCBTB2 RHBDD3 RNF208 RPS2 RYR1 PTS RABEP2 RASD1 RCC1 RHBDF1 RNF214 RPS26 RYR2 PTTG1 RABGAP1 RASD2 RCC2 RHBDF2 RNF216 RPS5 RYR3 PUF60 RABGEF1 RASGEF1B RCE1 RHBDL1 RNF219 RPS6 S100A1 PUM2 RABGGTA RASGEF1C RCHY1 RHBDL3 RNF220 RPS6KA4 S100A14

PURA RABGGTB RASGRF1 RCN2 RHCG RNF24 RPS6KA5 S100A16 PURG RABL3 RASGRF2 RCOR1 RHO RNF26 RPS6KA6 S100A4 PUS1 RABL5 RASGRP1 RCOR2 RHOBTB1 RNF31 RPS6KB1 S100P PUS10 RABL6 RASGRP2 RCOR3 RHOBTB3 RNF34 RPS6KB2 S100PBP PUS7 RAC1 RASL11A RDH12 RHOC RNF38 RPS7 S1PR2 PVRL1 RAC2 RASL11B RDH16 RHOH RNF40 RPS8 S1PR4 PVRL4 RAC3 RASL12 RDX RHOJ RNF41 RPS9 SACM1L PWP2 RACGAP1 RASSF2 REC8 RHOQ RNF44 RPTOR SACS PXDN RAD17 RASSF8 REEP2 RHOT1 RNF5 RPUSD1 SAE1 PXMP2 RAD21 RAVER1 REEP5 RHOU RNF8 RPUSD2 SAFB PXN RAD23B RAVER2 REEP6 RHPN1 RNFT2 RQCD1 SALL1 PYCR1 RAD50 RB1 REG1A RIBC1 RNGTT RRAD SALL4 PYCR2 RAD51 RB1CC1 REG3A RIC8A RNH1 RRAGA SAMD13 PYGB RAD54L RBBP4 REL RIC8B RNPEP RRAGB SAMD14 PYGM RAD54L2 RBBP5 RELA RICTOR RNPS1 RRAGC SAMD3 PYGO2 RAD9A RBBP6 RELN RIF1 ROBO1 RRAGD SAMD4A PYROXD1 RAE1 RBBP7 REM1 RILPL2 ROBO2 RREB1 SAMD8 QARS RAF1 RBBP8 REM2 RIMBP2 ROCK1 RRM1 SAMHD1 QDPR RAG1 RBBP9 REN RIMKLA ROGDI RRM2 SAMM50 QKI RAI1 RBFOX1 REPS1 RIMS1 ROM1 RRM2B SAP130 QRICH1 RAI14 RBFOX2 REPS2 RIMS2 ROR1 RRNAD1 SAP18 QSER1 RAI2 RBFOX3 RER1 RIMS4 RORB RRP1B SAP30BP QSOX1 RALB RBL1 RERE RING1 RPAP2 RRP9 SAR1B QSOX2 RALBP1 RBL2 RERG RINT1 RPAP3 RSBN1 SARNP QTRT1 RALGAPA1 RBM10 RET RIOK3 RPE RSBN1L SARS R3HDM1 RALGAPA2 RBM12B REV3L RIPK1 RPE65 RSG1 SARS2 R3HDM2 RALGDS RBM14 REXO1 RIPK4 RPH3A RSL1D1 SASH1 RAB11A RALGPS1 RBM14-RBM4 REXO2 RIT1 RPH3AL RSL24D1 SASH3 RAB11B RALGPS2 RBM15B RFC2 RLIM RPIA RSPH9 SASS6 RAB11FIP4 RALY RBM17 RFC3 RLN1 RPL10A RSPO3 SATB1 RAB11FIP5 RALYL RBM18 RFC4 RMND5B RPL11 RSPRY1 SATB2 
RAB12 RAMP1 RBM22 RFC5 RNASE12 RPL13A RSRC1 SAV1 RAB19 RAMP2 RBM23 RFFL RNASEH1 RPL15 RSRC2 SBF1 RAB1B RAN RBM25 RFK RNASEH2C RPL17 RSU1 SBF2 RAB20 RANBP1 RBM26 RFTN1 RNASEL RPL18 RTEL1 SBNO1 RAB22A RANBP10 RBM27 RFWD2 RND2 RPL18A RTF1 SBNO2 RAB24 RANBP2 RBM3 RFWD3 RND3 RPL23A RTKN SC5DL RAB26 RANBP3 RBM34 RFX1 RNF10 RPL24 RTKN2 SCAF11 RAB27A RANBP9 RBM39 RFX2 RNF103 RPL26L1 RTN2 SCAF4 RAB28 RANGAP1 RBM4 RFX3 RNF103-CHMP3 RPL27 RTN3 SCAI RAB30 RANGRF RBM41 RFX4 RNF115 RPL29 RTN4IP1 SCAMP2 RAB31 RAP1A RBM42 RGL2 RNF121 RPL3 RUFY1 SCAMP5 RAB32 RAP1B RBM45 RGL3 RNF126 RPL34 RUFY2 SCAP RAB34 RAP1GAP RBM46 RGMA RNF130 RPL6 RUFY3 SCARA3 RAB35 RAPGEF1 RBM47 RGMB RNF139 RPL7 RUNDC1 SCARB1 RAB3A RAPGEF2 RBM48 RGNEF RNF14 RPL7A RUNDC3A SCD RAB3C RAPGEF3 RBM4B RGP1 RNF144A RPL7L1 RUNDC3B SCFD1 RAB3IL1 RAPGEF4 RBM6 RGR RNF145 RPL8 RUNX1 SCG2 RAB3IP RAPGEF6 RBM7 RGS1 RNF148 RPL9 RUNX2 SCG3 RAB40B RAPGEFL1 RBM8A RGS10 RNF149 RPLP1 RUNX3 SCG5 SCGB2A1 SEPT10 SGPP1 SLC12A7 SLC26A6 SLC4A3 SMAP2 SNX14 SCGB2A2 SEPT11 SGPP2 SLC13A2 SLC26A9 SLC4A4 SMARCA1 SNX18 SCHIP1 SEPT12 SGSM1 SLC13A3 SLC27A1 SLC4A5 SMARCA2 SNX2 SCIMP SEPT3 SGSM2 SLC13A4 SLC27A2 SLC4A8 SMARCA4 SNX24 SCLT1 SEPT4 SGSM3 SLC13A5 SLC27A3 SLC4A9 SMARCAL1 SNX25 SCMH1 SEPT5 SGTA SLC15A2 SLC27A6 SLC50A1 SMARCB1 SNX29 SCML1 SEPT6 SH2B1 SLC16A1 SLC29A2 SLC5A10 SMARCC1 SNX30 SCML2 SEPT8 SH2D3C SLC16A12 SLC2A13 SLC5A12 SMARCC2 SNX32 SCN10A SEPT9 SH2D5 SLC16A13 SLC2A14 SLC5A2 SMARCD1 SNX33 SCN11A SERBP1 SH3BP2 SLC16A14 SLC2A2 SLC5A3 SMARCD2 SNX4 SCN1A SERINC3 SH3BP4 SLC16A3 SLC2A4 SLC5A5 SMARCD3 SNX5 SCN2A SERINC5 SH3BP5L SLC16A5 SLC2A5 SLC5A6 SMC1A SNX6 SCN2B SERPINA3 SH3D19 SLC16A6 SLC2A6 SLC6A1 SMC2 SNX9 SCN3A SERPINA4 SH3GL2 SLC17A6 SLC30A1 SLC6A11 SMC3 SOAT1 SCN3B SERPINA6 SH3GLB1 SLC17A7 SLC30A10 SLC6A12 SMC5 SOCS4 SCN4A SERPINB13 SH3KBP1 SLC18A2 SLC30A3 SLC6A13 SMC6 SOD1 SCN4B SERPINB4 SH3PXD2A SLC18A3 SLC30A4 SLC6A14 SMCHD1 SOGA2

SCN5A SERPINB6 SH3PXD2B SLC18B1 SLC30A5 SLC6A16 SMCR7 SOGA3

SCN8A SERPINB7 SH3RF1 SLC19A1 SLC30A7 SLC6A17 SMCR8 SON SCO1 SERPINB9 SH3RF2 SLC19A2 SLC31A2 SLC6A19 SMEK1 SORBS2 SCO2 SERPINE1 SHC1 SLC19A3 SLC34A1 SLC6A2 SMEK2 SORCS2 SCP2 SERPINE2 SHC4 SLC1A1 SLC35A2 SLC6A20 SMG1 SORL1 SCPEP1 SERPINF1 SHCBP1 SLC1A2 SLC35B1 SLC6A3 SMG6 SOWAHB 
SCRIB SERPINF2 SHE SLC1A3 SLC35B2 SLC6A4 SMG7 SOX10 SCUBE1 SERPING1 SHISA3 SLC1A4 SLC35B3 SLC6A6 SMG8 SOX14 SCUBE2 SERPINH1 SHISA5 SLC1A6 SLC35B4 SLC6A7 SMG9 SOX2 SCUBE3 SERTAD2 SHMT2 SLC1A7 SLC35C2 SLC6A8 SMO SOX7 SDC4 SERTAD3 SHOC2 SLC20A1 SLC35D1 SLC6A9 SMOC2 SOX9 SDCBP SESN1 SHOX2 SLC20A2 SLC35D2 SLC7A1 SMPD2 SP2 SDCCAG8 SESN2 SHPK SLC22A13 SLC35E1 SLC7A10 SMPD3 SP3 SDF4 SESN3 SHROOM1 SLC22A17 SLC35E2B SLC7A3 SMPD4 SP4 SDHA SESTD1 SIAH2 SLC22A2 SLC35E3 SLC7A7 SMS SP7 SDK1 SETD1A SIAH3 SLC22A23 SLC35E4 SLC7A8 SMU1 SPACA1 SDK2 SETD4 SIGLEC1 SLC22A3 SLC35F3 SLC7A9 SMURF1 SPAG5 SDS SETD5 SIGLEC10 SLC22A4 SLC35G1 SLC9A1 SMURF2 SPAST SEC13 SETD9 SIGLEC7 SLC22A6 SLC35G2 SLC9A2 SMYD1 SPATA12 SEC14L1 SETDB1 SIK1 SLC22A8 SLC35G3 SLC9A3 SMYD3 SPATA17 SEC14L5 SETDB2 SIK2 SLC23A3 SLC36A1 SLC9A5 SNAI2 SPATA19 SEC16A SETX SIKE1 SLC24A1 SLC37A1 SLC9A6 SNAP23 SPATA2 SEC22A SEZ6L SIM1 SLC24A2 SLC37A3 SLC9A7 SNAP91 SPATA20 SEC22C SF1 SIM2 SLC24A5 SLC38A1 SLC9A8 SNAPC3 SPATA5 SEC23A SF3A1 SIN3A SLC25A1 SLC38A5 SLC9A9 SNAPC5 SPATA5L1 SEC24B SF3A3 SIN3B SLC25A10 SLC38A6 SLCO1A2 SNCA SPATA6 SEC61A1 SF3B4 SIPA1 SLC25A11 SLC38A7 SLCO2B1 SNCB SPATS2 SEC61A2 SFMBT1 SIPA1L1 SLC25A12 SLC39A1 SLCO3A1 SNED1 SPDYC SEC61B SFMBT2 SIPA1L2 SLC25A14 SLC39A10 SLCO4A1 SNF8 SPECC1 SEC63 SFN SIPA1L3 SLC25A16 SLC39A14 SLCO4C1 SNN SPECC1L SECISBP2 SFR1 SIRPA SLC25A18 SLC39A5 SLIT1 SNPH SPEG SEH1L SFRP1 SIRT4 SLC25A19 SLC39A6 SLIT2 SNRK SPEN SEL1L3 SFRP2 SIRT7 SLC25A20 SLC3A1 SLIT3 SNRNP200 SPG11 SELENBP1 SFRP4 SIX1 SLC25A24 SLC3A2 SLITRK1 SNRNP25 SPG20 SELM SFT2D2 SKIL SLC25A25 SLC41A2 SLITRK2 SNRNP35 SPG21 SELO SFXN2 SKP2 SLC25A26 SLC43A1 SLITRK3 SNRNP40 SPHK1 SELT SFXN4 SLA SLC25A28 SLC43A3 SLITRK4 SNRNP48 SPHKAP SEMA3C SFXN5 SLAIN1 SLC25A3 SLC44A1 SLITRK5 SNRPA SPI1 SEMA3D SGCA SLAIN2 SLC25A30 SLC44A5 SLITRK6 SNRPB SPIB SEMA3E SGCB SLAMF6 SLC25A31 SLC45A1 SLK SNRPB2 SPIC SEMA3F SGCD SLC10A1 SLC25A33 SLC45A3 SLMO1 SNRPD2 SPIN3 SEMA3G SGCE SLC10A2 SLC25A34 SLC45A4 SLPI SNRPN SPIRE2 SEMA4C SGCG SLC10A3 SLC25A35 SLC47A1 SLU7 SNTA1 SPN SEMA5A SGCZ SLC11A1 SLC25A36 SLC47A2 SMAD1 SNTB1 SPNS1 SEMA5B SGK1 SLC12A1 SLC25A42 SLC4A1 SMAD2 SNW1 SPNS3 SEMA6A SGK2 SLC12A2 SLC25A44 SLC4A10 SMAD3 SNX1 SPOCK1 SEMA6D SGK3 SLC12A3 SLC25A51 SLC4A11 SMAD4 SNX10 SPOCK2 SENP2 SGMS1 SLC12A4 SLC25A6 SLC4A1AP SMAD9 SNX11 SPOP SEPHS1 SGPL1 SLC12A5 SLC26A5 SLC4A2 SMAP1 SNX13 SPP2 SPPL3 SSU72 STRADB SYNJ2 TBC1D15 TESC TIE1 TMEM136 
SPRED1 SSX2IP STRAP SYNPO TBC1D16 TESK1 TIFAB TMEM138 SPRED2 ST14 STRBP SYNPO2 TBC1D17 TET3 TIGD2 TMEM143 SPRTN ST18 STRN SYNPO2L TBC1D19 TEX10 TIGD3 TMEM145 SPRY1 ST3GAL2 STRN3 SYNPR TBC1D20 TEX11 TIGD7 TMEM147 SPRY3 ST3GAL3 STRN4 SYNRG TBC1D21 TEX14 TIGIT TMEM14B SPRYD3 ST3GAL5 STS SYP TBC1D22B TEX261 TIMM17B TMEM150A SPRYD7 ST3GAL6 STT3A SYPL1 TBC1D23 TEX29 TIMM21 TMEM150B SPSB1 ST5 STT3B SYT1 TBC1D7 TEX30 TIMM8B TMEM161A SPSB2 ST6GAL1 STUB1 SYT10 TBC1D9B TEX34 TIMP2 TMEM163 SPSB3 ST6GALNAC3 STX10 SYT11 TBCB TEX9 TIMP3 TMEM164 SPTAN1 ST6GALNAC4 STX12 SYT12 TBCD TF TIMP4 TMEM165 SPTB ST6GALNAC5 STX16 SYT13 TBCEL TFAP2A TINAGL1 TMEM168 SPTBN1 ST7 STX1A SYT14 TBK1 TFAP2B TIPARP TMEM169 SPTBN2 ST8SIA2 STX1B SYT17 TBL1X TFAP2C TJP1 TMEM170B SPTBN4 ST8SIA3 STX4 SYT2 TBL1XR1 TFB2M TK1 TMEM179 SPTLC1 ST8SIA4 STX5 SYT3 TBL3 TFCP2 TK2 TMEM179B SPTLC2 ST8SIA5 STX6 SYT6 TBP TFCP2L1 TKT TMEM180 SPTLC3 STAB1 STX7 SYT7 TBR1 TFDP2 TKTL1 TMEM182 SPTSSA STAB2 STXBP1 SYT9 TBRG1 TFEB TKTL2 TMEM184A SQLE STAC STXBP3 SYTL1 TBRG4 TFG TLE1 TMEM184B SQSTM1 STAC3 STXBP5 SYVN1 TBX10 TFIP11 TLE4 TMEM184C SRC STAG2 STXBP5L SZRD1 TBX18 TFPT TLE6 TMEM186 SRCAP STAM STXBP6 TAAR1 TBX20 TFR2 TLK1 TMEM189 SRD5A1 STAM2 STYX TAB1 TBX21 TFRC TLK2 TMEM189-UBE2V1 SREBF1 STAP1 SUCLA2 TAB3 TBX22 TGDS TLN1 TMEM190 SREBF2 STAP2 SUCLG1 TACC1 TBX3 TGFA TLN2 TMEM192 SREK1 STAR SUCNR1 TACO1 TBX4 TGFB1 TLR2 TMEM2 SRF STARD10 SUCO TACR1 TBX5 TGFB1I1 TLR3 TMEM200A SRGAP1 STARD13 SUDS3 TACR3 TBXA2R TGFB3 TLR7 TMEM203 SRGAP3 STARD3 SUFU TADA1 TC2N TGFBI TLR8 TMEM204 SRM STARD3NL SUGT1 TADA2B TCEA2 TGFBR1 TLR9 TMEM206 SRP19 STARD4 SULF1 TADA3 TCEA3 TGIF2 TLX3 TMEM208 SRP54 STAT1 SULF2 TAF1 TCEAL6 TGM1 TM4SF1 TMEM214 SRP72 STAT2 SULT1A1 TAF13 TCERG1 TGM2 TM4SF18 TMEM219 SRP9 STAT3 SULT1E1 TAF15 TCF12 TGM3 TM4SF4 TMEM222 SRPK1 STAT4 SULT4A1 TAF1L TCF20 TH1L TM4SF5 TMEM245 SRPK2 STAT5A SUMF1 TAF5 TCF3 THAP1 TM6SF1 TMEM246 SRPK3 STAT5B SUN5 TAF6 TCF4 THAP10 TM7SF2 TMEM248 SRPR STAT6 SUPT16H TAF6L TCF7L2 THAP11 TM7SF3 TMEM26 SRR STAU1 SUPT5H TAF7 TCHP THAP4 TM9SF2 TMEM27 SRRD STC1 SUPT6H TAF8 TCP1 THAP5 TM9SF3 TMEM30A SRRM2 STC2 SUPT7L TAF9 TCTN2 THBS1 TM9SF4 TMEM33 SRRT STEAP1 SURF1 TAF9B TDP1 THBS2 TMBIM6 TMEM38A SRSF11 STEAP3 SURF4 TAGLN2 TDRD1 THBS3 TMC1 TMEM39A 
SRSF2 STIM1 SUSD1 TALDO1 TDRD3 THBS4 TMCC1 TMEM39B SRSF5 STIM2 SUSD4 TAMM41 TDRD6 THEG TMCO3 TMEM40 SRSF6 STIP1 SUV39H1 TANC1 TDRD7 THEMIS TMED1 TMEM45A SRSF7 STK11 SUV39H2 TANC2 TDRD9 THEMIS2 TMED2 TMEM48 SRSF9 STK11IP SUZ12 TAOK1 TEAD1 THG1L TMED4 TMEM51 SS18 STK16 SV2A TAOK2 TEAD3 THOC1 TMED8 TMEM55B SS18L1 STK24 SV2B TAOK3 TEC THOC2 TMEFF1 TMEM56 SSB STK3 SV2C TARBP1 TECPR1 THOC7 TMEFF2 TMEM57 SSBP1 STK32A SVIL TARBP2 TECPR2 THOP1 TMEM100 TMEM59 SSBP3 STK32B SVOPL TARDBP TECR THPO TMEM101 TMEM62 SSBP4 STK32C SWAP70 TARS2 TECTA THRA TMEM108 TMEM63C SSH1 STK33 SYAP1 TARSL2 TECTB THRAP3 TMEM109 TMEM64 SSH2 STK38 SYBU TAS1R3 TEF THRB TMEM11 TMEM66 SSH3 STK38L SYCP3 TAS2R10 TEFM THSD7A TMEM115 TMEM72 SSR2 STK39 SYK TASP1 TEK THTPA TMEM117 TMEM74 SSR3 STK4 SYMPK TAT TEKT2 THUMPD1 TMEM120B TMEM74B SSRP1 STMN3 SYN3 TAX1BP1 TENC1 THUMPD2 TMEM127 TMEM87A SSTR1 STOML1 SYNCRIP TBC1D10A TEPP THYN1 TMEM129 TMEM87B SSTR2 STOML2 SYNE1 TBC1D10C TERF1 TIAL1 TMEM132A TMEM8B SSTR4 STON2 SYNGR1 TBC1D13 TERF2IP TIAM2 TMEM132B TMEM8C SSTR5 STOX2 SYNGR2 TBC1D14 TES TICAM1 TMEM132D TMEM9 TMEM91 TOP2B TRIM17 TSN TUFM UBXN4 USP31 VPS37B TMEM95 TOP3B TRIM21 TSNAXIP1 TULP4 UBXN6 USP32 VPS39 TMEM97 TOPBP1 TRIM23 TSPAN11 TUSC3 UBXN7 USP33 VPS41 TMEM9B TOR1A TRIM24 TSPAN12 TUT1 UCHL1 USP34 VPS45 TMF1 TOR1AIP2 TRIM25 TSPAN13 TWF2 UCHL3 USP38 VPS4A TMIE TOR1B TRIM26 TSPAN14 TWISTNB UCK2 USP39 VPS51 TMIGD1 TOR3A TRIM27 TSPAN17 TWSG1 UCKL1 USP4 VPS52 TMLHE TOX TRIM29 TSPAN3 TXK UCN2 USP43 VPS53 TMOD1 TOX2 TRIM3 TSPAN33 TXLNA UEVLD USP44 VPS54 TMOD2 TOX4 TRIM32 TSPAN5 TXN2 UFC1 USP46 VPS8 TMOD3 TP53 TRIM33 TSPAN6 TXNDC15 UFL1 USP47 VRK1 TMPPE TP53BP1 TRIM35 TSPAN7 TXNL4A UGCG USP48 VRTN TMPRSS11B TP53I11 TRIM37 TSPAN9 TXNRD1 UGDH USP5 VSIG1 TMPRSS11D TP53I13 TRIM41 TSPYL2 TYK2 UGT1A1 USP51 VSTM2A TMPRSS3 TP53INP1 TRIM44 TSSK1B TYMS UGT1A10 USP6NL VSTM4 TMTC1 TP73 TRIM46 TSSK2 TYRO3 UGT1A6 USP7 VSX2 TMTC4 TPBG TRIM50 TSSK3 TYRP1 UGT1A7 USP9X VTI1A TMUB2 TPCN2 TRIM52 TSSK6 TYW1 UGT1A8 UST VTI1B TMX1 TPH1 TRIM54 TTBK1 TYW5 UGT1A9 UTRN VTN TMX2 TPH2 TRIM55 TTBK2 U2AF1 UGT2B7 UVRAG VWCE TNC TPI1 TRIM62 TTC12 U2AF1L4 UHMK1 UXS1 WAC TNFAIP3 TPM1 TRIM63 TTC13 UACA UHRF2 UXT WAPAL TNFAIP8 TPM2 TRIM8 TTC17 UAP1L1 ULK1 VAMP2 WARS 
TNFAIP8L2 TPM4 TRIM9 TTC23 UBA1 ULK3 VAMP3 WASL TNFRSF11B TPO TRIO TTC26 UBA2 UMOD VAMP5 WBP1 TNFRSF12A TPPP TRIP10 TTC27 UBA3 UMPS VANGL1 WBP11 TNFRSF6B TPPP3 TRIP12 TTC32 UBAC2 UNC119 VARS WBP4 TNFSF11 TPR TRIP13 TTC39A UBAP1 UNC13A VASH1 WBSCR17 TNFSF12 TPRG1L TRMT1 TTC39B UBASH3A UNC13B VASP WBSCR22 TNFSF12-TNFSF13 TPRKB TRMT11 TTC39C UBASH3B UNC13C VAT1 WDFY1

TNFSF14 TPRX1 TRMT112 TTC7B UBB UNC5A VAT1L WDFY3 TNFSF18 TPST1 TRMT1L TTC9C UBC UNC5B VAV1 WDR1 TNFSF4 TPST2 TRMT2B TTI1 UBE2C UNC5C VAV2 WDR12 TNFSF8 TPT1 TRMT6 TTK UBE2E2 UNC5D VAV3 WDR13 TNIK TPTE2 TRMT61A TTL UBE2F UNG VAX1 WDR16 TNIP2 TPX2 TRMU TTLL1 UBE2G2 UPB1 VCAN WDR20 TNK2 TRA2A TRNAU1AP TTLL11 UBE2I UPF1 VCL WDR24 TNKS TRA2B TRNT1 TTLL12 UBE2J1 UPF2 VCP WDR26 TNKS2 TRABD TROVE2 TTLL13 UBE2J2 UPP1 VCPIP1 WDR3 TNMD TRAF2 TRPC1 TTLL3 UBE2L6 UPP2 VDAC1 WDR33 TNNC1 TRAF3 TRPC3 TTLL6 UBE2O UPRT VDAC2 WDR34 TNNI2 TRAF4 TRPC4 TTLL7 UBE2Q1 UQCRC1 VDR WDR43 TNNI3K TRAF6 TRPC4AP TTPA UBE2R2 UQCRFS1 VEZF1 WDR44 TNNT1 TRAF7 TRPC5 TTYH3 UBE2S URB2 VGLL1 WDR45 TNNT2 TRAFD1 TRPC6 TUB UBE2T URGCP VILL WDR45L TNNT3 TRAIP TRPC7 TUBA1A UBE2Z URI1 VIM WDR46 TNPO1 TRAK1 TRPM5 TUBA1B UBE3A URM1 VIMP WDR47 TNPO3 TRAK2 TRPM6 TUBA1C UBE3B UROS VIP WDR48 TNR TRAM1 TRPM7 TUBA3C UBE4B USE1 VIPAS39 WDR5 TNRC6B TRAM1L1 TRPM8 TUBA3D UBIAD1 USF1 VIPR1 WDR53 TNRC6C TRAM2 TRPS1 TUBA4A UBL4A USH1C VIPR2 WDR59 TNS4 TRANK1 TRPV2 TUBA8 UBL7 USHBP1 VIT WDR6 TOB1 TRAP1 TRPV4 TUBAL3 UBLCP1 USP1 VMP1 WDR61 TOB2 TRAPPC1 TRPV6 TUBB UBN1 USP10 VPS13A WDR63 TOE1 TRAPPC10 TRRAP TUBB2A UBN2 USP12 VPS13B WDR7 TOLLIP TRAPPC12 TSC2 TUBB3 UBOX5 USP14 VPS13C WDR70 TOM1 TRAPPC2L TSC22D1 TUBB4A UBP1 USP16 VPS13D WDR74 TOM1L1 TRAPPC4 TSC22D3 TUBB6 UBQLN1 USP19 VPS16 WDR75 TOMM22 TRAPPC6B TSEN2 TUBD1 UBQLN2 USP20 VPS18 WDR77 TOMM34 TRAPPC9 TSG101 TUBG1 UBR1 USP21 VPS26A WDR81 TOMM40 TREX1 TSGA10 TUBG2 UBR2 USP22 VPS26B WDR83 TOMM40L TRHR TSGA13 TUBGCP2 UBR4 USP24 VPS28 WDR89 TOMM70A TRIB2 TSHZ1 TUBGCP3 UBR5 USP25 VPS33B WDR91 TONSL TRIM11 TSHZ3 TUBGCP4 UBTD1 USP28 VPS35 WDR96 TOP1 TRIM13 TSKS TUBGCP5 UBXN10 USP3 VPS36 WDTC1 TOP1MT TRIM16L TSLP TUBGCP6 UBXN2B USP30 VPS37A WEE2 
WFDC2 XPO5 ZBTB39 ZFP1 ZNF19 ZNF35 ZNF555 ZNF74 WFDC6 XPO6 ZBTB4 ZFP106 ZNF2 ZNF354A ZNF557 ZNF746 WFIKKN2 XPOT ZBTB40 ZFP161 ZNF202 ZNF383 ZNF560 ZNF75A WFS1 XPR1 ZBTB41 ZFP3 ZNF205 ZNF384 ZNF563 ZNF75D WHSC1 XRCC5 ZBTB43 ZFP36 ZNF207 ZNF385A ZNF564 ZNF766 WHSC1L1 XRCC6 ZBTB44 ZFP36L1 ZNF213 ZNF391 ZNF567 ZNF768 WIBG XRN1 ZBTB45 ZFP82 ZNF217 ZNF394 ZNF569 ZNF770 WIF1 XRN2 ZBTB46 ZFP90 ZNF22 ZNF395 ZNF570 ZNF773 WIPF1 XYLT2 ZBTB5 ZFR ZNF226 ZNF397 ZNF574 ZNF776 WIZ YAP1 ZBTB6 ZFX ZNF23 ZNF398 ZNF575 ZNF786 WLS YARS ZBTB7B ZFYVE1 ZNF232 ZNF410 ZNF581 ZNF791 WNK3 YARS2 ZBTB7C ZFYVE20 ZNF236 ZNF416 ZNF582 ZNF8 WNT10B YBX2 ZBTB8A ZFYVE26 ZNF238 ZNF423 ZNF583 ZNF814 WNT11 YEATS4 ZC3H10 ZFYVE28 ZNF239 ZNF425 ZNF584 ZNF816ZNF321P

WNT2 YES1 ZC3H11A ZGPAT ZNF248 ZNF426 ZNF589 ZNF821 WNT2B YIF1A ZC3H12B ZHX1 ZNF25 ZNF432 ZNF592 ZNF827 WNT3 YIF1B ZC3H12C ZHX1-C8ORF76 ZNF250 ZNF433 ZNF606 ZNF829 WNT3A YIPF1 ZC3H13 ZHX3 ZNF251 ZNF434 ZNF607 ZNF836 WNT4 YIPF2 ZC3H14 ZIC1 ZNF253 ZNF436 ZNF608 ZNF862 WNT5A YIPF3 ZC3H15 ZIK1 ZNF260 ZNF445 ZNF609 ZNFX1 WNT5B YIPF6 ZC3H18 ZKSCAN1 ZNF263 ZNF449 ZNF618 ZNHIT1 WNT7A YIPF7 ZC3H4 ZKSCAN4 ZNF266 ZNF451 ZNF619 ZNHIT2 WNT8A YJEFN3 ZC3H6 ZKSCAN5 ZNF276 ZNF460 ZNF621 ZNHIT3 WNT8B YKT6 ZC3H7A ZMAT3 ZNF280C ZNF461 ZNF622 ZNHIT6 WNT9A YLPM1 ZC3H7B ZMIZ1 ZNF280D ZNF462 ZNF624 ZNRF3 WRAP53 YME1L1 ZCCHC11 ZMIZ2 ZNF281 ZNF48 ZNF627 ZP2 WRAP73 YOD1 ZCCHC12 ZMPSTE24 ZNF282 ZNF480 ZNF638 ZP4 WSB1 YTHDC1 ZCCHC14 ZMYM2 ZNF287 ZNF483 ZNF644 ZPBP WSCD1 YTHDC2 ZCCHC6 ZMYM3 ZNF295 ZNF485 ZNF652 ZPLD1 WSCD2 YTHDF1 ZCCHC7 ZMYM6 ZNF296 ZNF490 ZNF653 ZRANB1 WT1 YWHAE ZDHHC1 ZMYND11 ZNF3 ZNF493 ZNF668 ZRANB2 WTAP YWHAG ZDHHC15 ZMYND19 ZNF300 ZNF496 ZNF670 ZSCAN10

WWC1 YWHAH ZDHHC17 ZMYND8 ZNF318 ZNF498 ZNF671 ZSCAN2 WWP1 YWHAQ ZDHHC18 ZNF10 ZNF319 ZNF500 ZNF677 ZSCAN20 WWP2 YY2 ZDHHC21 ZNF114 ZNF32 ZNF511 ZNF687 ZSCAN21 XAB2 ZADH2 ZDHHC22 ZNF12 ZNF320 ZNF512 ZNF688 ZSCAN22 XBP1 ZBED1 ZDHHC23 ZNF121 ZNF324 ZNF512B ZNF689 ZSCAN29 XK ZBED4 ZDHHC5 ZNF124 ZNF324B ZNF513 ZNF691 ZSCAN4 XKR4 ZBTB1 ZDHHC7 ZNF132 ZNF326 ZNF514 ZNF692 ZSCAN5A XKR6 ZBTB10 ZDHHC9 ZNF134 ZNF329 ZNF521 ZNF699 ZSWIM3 XKR7 ZBTB11 ZEB2 ZNF136 ZNF331 ZNF526 ZNF70 ZSWIM4 XKR8 ZBTB16 ZFAND1 ZNF142 ZNF33B ZNF527 ZNF704 ZSWIM5 
XPNPEP1 ZBTB17 ZFAND5 ZNF143 ZNF34 ZNF529 ZNF709 ZSWIM7 XPNPEP2 ZBTB24 ZFAT ZNF148 ZNF345 ZNF532 ZNF710 ZXDC XPNPEP3 ZBTB25 ZFC3H1 ZNF17 ZNF346 ZNF536 ZNF711 ZZEF1 XPO1 ZBTB26 ZFHX3 ZNF174 ZNF347 ZNF547 ZNF713 ZZZ3 XPO4 ZBTB34 ZFHX4 ZNF18

\subsection{Attachment 11}

ABCC8 CARF EPS8L1 IGLON5 MIR124-2HG PLCB4 SASH1 SYT13 ABCD2 CARMIL2 ERC1 INPP5F MIR22HG PLEKHA1 SCAMP5 SYT16 ABCG4 CARMIL3 ERC2 IQSEC1 MIR3936HG PLK5 SCARA3 SYT3 ABLIM2 CASKIN1 ETNK2 IRAK2 MMD PLPPR2 SCML1 SYT5 ACKR1 CCDC149 FADS3 IRX4 MMP24 PLPPR4 SCN2B SYT7 ACKR3 CCDC184 FAHD2B JAG2 MOAP1 PLXDC1 SCN3B TACR3 ACOT7 CCDC186 FAM102B JAK1 MPP1 PLXNC1 SCN8A TATDN2P2 ACTL6B CDH10 FAM131C JAKMIP1 MPP3 PNCK SCN9A TBC1D24 ACYP1 CDH12 FAM172A JAKMIP3 MTMR6 PNMA2 SELENBP1 TBC1D9 ADAM11 CDH9 FAM196A JPH3 MTND6P21 PNMA8B SEMA4F TCAF1 ADCY5 CDK5R2 FAM43B KCNA2 MTURN PPP1R13B SEMA5A TCAF1P1 ADD2 CDKN2D FAM57B KCNA3 MYT1L PPP1R16B SEMA7A TDRD7 ADD3 CELF5 FAXDC2 KCNA4 N4BP3 PPP1R1A SESTD1 TFPI ADGRB1 CEP126 FBXL16 KCNAB2 NANOS1 PPP1R3B SEZ6L2 TFR2 ADGRL1 CEP170B FBXL2 KCNB1 NBEAL2 PRKACA SH3GL3 TIAF1 ADGRV1 CGREF1 FBXL4 KCNC1 NCAM2 PRKCE SH3KBP1 TMCC2 ADRA1B CHD5 FBXO2 KCNC3 NCOA3 PRKCZ SH3PXD2A TMEM107 ADRA2A CHGA FBXO27 KCNC4 NCS1 PRKN SH3RF1 TMEM130 AGAP1 CHRNA4 FBXO41 KCNH7 NDRG4 PRNP SHANK1 TMEM143 AGAP2 CKMT1A FBXO44 KCNJ11 NECAB2 PRRT2 SHANK3 TMEM145 AHNAK2 CKMT1B FGF12 KCNK3 NECTIN1 PRRT3 SIPA1 TMEM151A ALDOC CLSTN3 FGF19 KCNN1 NEDD4L PRRT4 SLC12A5 TMEM169 AMER1 CNTN1 FHOD3 KCNQ3 NEK7 PSD SLC23A2 TMEM229B AMER3 CORO6 FLRT3 KHK NENF PSD2 SLC24A3 TMEM246 ANK2 CPEB4 FMNL1 KIAA0513 NEURL1 PSD3 SLC25A27 TMEM44 ANKRD24 CPLX1 FNBP1P1 KIAA1024 NFASC PTGDS SLC29A1 TMEM54

AP3B2 CPNE5 FNDC10 KIAA1549L NHS PTGR2 SLC2A10 TMEM74B AQP11 CPNE7 FOXC2 KIF16B NIPAL3 PTPN5 SLC2A12 TMOD2 ARHGAP20 CRB1 FRS3 KIF21B NMNAT3 PTPRN SLC36A1 TPPP ARHGAP33 CREG2 FRY KLF3-AS1 NOVA1 PTPRN2 SLC38A3 TRIM3 ARHGDIG CTSH FSTL5 KLF9 NPC1 RAB11FIP2 SLC46A3 TRUB1 ARL2 CYGB FXYD7 KLHL15 NPIPA5 RAB11FIP4 SLC4A10 TSPOAP1 ATG16L2 DAB1 GABBR1 KLHL32 NPM2 RAB11FIP5 SLC6A17 TTBK1 
ATP1A3 DACH2 GABRG2 KNDC1 NRG2 RAB26 SLC8A3 TTC14 ATP2B2 DACT3 GALNT14 L1CAM NRSN1 RAB39A SLC9A3R2 TTC9B ATP2B3 DFNB59 GALNT9 L3MBTL1 NRXN2 RAB3A SLC9A5 TUBB2A ATRNL1 DHRS13 GDAP1L1 LDB2 NTNG1 RAB3B SLC9A9 TUBB4A B4GALNT1 DISP2 GDPD5 LGI3 NUTM2D RAB3IL1 SLITRK6 UBAP1L BCO2 DLGAP3 GLS2 LHFPL4 NYAP2 RAB6B SMAP1 UBE2Q2P1 BEX5 DMTN GNAL LIN7B OGDHL RABGAP1L SMIM10L2A UNC13A BICD1 DNAJC12 GNB5 LINC00265 OLFM1 RALGPS1 SMIM10L2B UNC5D

BICDL1 DNAJC6 GNG4 LINC02035 OPRL1 RAP1GAP SMKR1 UTRN BMP2K DNM1 GNPTAB LINC02298 OR1F1 RAP1GAP2 SMPD3 VAMP1 BNIP3 DOC2A GOLGA7B LINGO1 PACS1 RAPH1 SNCA VEGFA BOLA3-AS1 DTX1 GPRIN1 LMNTD2 PACSIN1 RASGRP2 SNCB VEGFB C15orf59 DUSP23 GPRIN2 LMTK2 PAG1 RASL11A SNCG VSTM2B C19orf66 DUSP5P1 GPRIN3 LRFN1 PAIP2B RASSF10 SNORA47 VWA5B2 C1QTNF4 ECE1 GREB1 LRRC3 PALM3 RASSF7 SNORD115-20 WASF3 C2orf27A EEF1A2 GRIK3 LRRTM3 PANX2 RBFOX3 SNPH WIPF3 C2orf72 EEF1AKMT2 GRIK5 LYNX1 PCDH1 RBM11 SNX10 WIPI1 C7orf31 EGFL7 H2AFJ LYSMD4 PCDHB13 RCAN2 SPACA9 WSCD2 C9orf172 EHHADH HCN2 MAGEE1 PCDHB15 REXO5 SPAG4 YBX2 CABLES2 EIF5A2 HCN3 MAOA PDE10A RGS2 SPHKAP ZBTB38 CABP1 ELAVL3 HIVEP2 MAP3K13 PDE1B RGS7BP SPRN ZBTB43 CACNA1B ELAVL4 HOXC5 MAP4K2 PDE2A RIC3 SPTBN4 ZCCHC12 CACNA1E ELFN2 HPCAL4 MAPK8IP2 PDIA2 RIMS4 SSPO ZDHHC4 CACNA2D3 ELMOD1 HPX MAPK9 PDXK RN7SL268P STAC2 ZFP28 CACNG2 EMID1 HRASLS MAPRE3 PDZD7 RNF180 STX1A ZFR2 CADM3 ENDOD1 HRH1 MAST1 PEBP1 RNF208 STX1B ZHX3 CADM3-AS1 ENO2 HRH3 MAST3 PEG10 RNU4-39P STX3 ZNF114 CALY ENPP5 HS6ST3 MAST4 PFKP RNY3P15 SWAP70 ZNF221 CAMK2B EPB41L2 HSPA12A MCF2L PID1 RP9P SYN1 ZNF382 CAMK2N1 EPB41L4B HTRA3 MCF2L-AS1 PIGZ RPL12P47 SYN2 ZNF467 CAMK2N2 EPHA10 IDS METTL7A PIK3CD RRN3P1 SYNGR1 ZNF540 CAMTA1 EPHA5 IFI44 MGAT3 PINK1 RSRC1 SYNGR3 ZNF547 CAPS2 EPHB6 IFNGR2 MGAT4A PIP5KL1 RUNDC3A-AS1 SYP ZNF699 ZNF774

\subsection{Attachment 12}

AAK1 CFH FAXC IDI1 MIR1273F PIK3CB RUSC1 SYT1 ABLIM3 CHRM2 FBN3 INA MMAB PJA2 SAMD12 SYT14 ACAP2-IT1 CISD1 FBXO25 ITGB1BP1 MRAS PKIA SCAI TAP2 ACSL3 CIT FCHSD2 JAZF1 MTMR7 PLCXD2 SCD TAS2R4 
ACSL4 CLASP2 FDFT1 KANTR MTSS1 PLPPR5 SCN1A TBC1D30 ACSL6 CLCN4 FDPS KBTBD11 MVD POLE SCN2A TBC1D8 ADAM22 CLVS2 FGD4 KCNAB1 MVK POMK SCN3A TCP11L1 ADAM23 CMBL FGF9 KCNB2 MYCBP2 POU2F1 SCUBE1 TENM1 ADCY1 CNNM1 FHDC1 KCND2 MYO5A POU2F2 SEMA4A TF ADGRL3 CNRIP1 FRRS1L KCNK1 MYO9A PPFIA2 SENP7 TLN2 AK5 CNTNAP2 FUT11 KCNK10 NAALAD2 PPP1R12B SGIP1 TM6SF1 AKAP9 COX6A1 GABBR2 KCNS2 NALCN PPP1R9A SGK1 TMEM135 ALDH5A1 CPB2-AS1 GABRA3 KIAA1107 NAP1L2 PPP2R2B SH3BP5 TMEM14A

AMPH CSMD3 GABRB2 KIAA1841 NAP1L3 PPP2R2C SHANK2 TMEM178B

ANK1 CSRNP3 GABRB3 KIF3A NAPB PPP2R3A SHISA7 TMEM196 ANK3 CTNNA2 GABRG3 KIFAP3 NAV3 PREPL SHROOM2 TMEM35A ANKRD12 CTSC GALNT13 KLHL1 NBEA PRKAA2 SKIL TMEM55A ANKRD18EP CTSO GFOD1 KSR2 NBEAL1 PRKAR1B SLC16A14 TMEM65

ANKRD36C CTTNBP2 GFRA2 LETMD1 NCOA2 PRKAR2B SLC1A1 TRAM1L1

ANKRD46 CYCSP34 GLRA1 LGI2 NCR3LG1 PRKCB SLC1A6 TRIB2

ANKRD55 CYP27A1 GLT1D1 LHFPL2 NELL1 PRUNE1 SLC25A12 TRIM67

APBA1 CYP46A1 GNAO1 LHFPL3 NETO1 PSPC1-AS2 SLC25A40 TRIQK APC DCX GNG2 LIN28B NEXMIF PTGR1 SLC2A13 TRMO

APH1B DDHD1 GNG3 LIN7C NFS1 PTPRR SLC2A6 TRMT2B

ARG2 DDX25 GNG8 LINC00599 NGLY1 PUS7L SLC30A4 TRPM3

ARMCX5-GPRASP2 DGKE GPC5 LINC00632 NLGN1 PWAR6 SLC43A2 TSPAN13

ARRB1 DHCR24 GPHN LINC00652 NMNAT2 PYGO1 SLC6A11 TSPAN7 ASTN1 DHCR7 GPI LINC01128 NRG3 RAB27B SLC6A1-AS1 TTC9 ASXL3 DNER GPLD1 LINC01184 NRG4 RAB3C SLC7A11 TTLL7 ATL1 DNM3 GPR12 LINC02293 NRXN1 RAB9B SLC8A1 UBASH3B ATP2B1-AS1 DOCK3 GPR21 LINC02486 NSDHL RALYL SLC9A6 UCHL5 ATP6V1G2 DPY19L2P2 GPR22 LINGO2 NUDT13 RAMP2-AS1 SLITRK1 UNC80

B4GALT6 DSCAM GPR85 LPGAT1 NXT2 RANBP17 SLITRK3 VEPH1 BNIP3L DSTNP1 GPRASP1 LPIN2 ODF2L RANBP3L SLITRK4 VN1R1 BRI3BP DYNC1I1 GPRASP2 LRBA OLFM3 RASA2 SMTNL2 VSNL1 C11orf87 EEF1AKMT1 GRB14 LRFN5 ONECUT2 RASAL2 SNAI3 WDR17 C14orf28 EFNA5 GRIA2 LRP2BP OXCT1 RBFA SNAI3-AS1 WDR7 C1R ELOVL4 GRIA3 LRRC28 P4HA1 REEP1 SNAP25 WEE2-AS1 C1S ELOVL6 GRIA4 LRRC3B PAK3 RFK SNAP91 XPR1 C9orf40 ENHO GRIN1 LRRC4C PAK5 RGS17 SOS1-IT1 ZBTB25 CA10 ENTPD1 GRIN2B LSAMP PALM2 RIMKLA SRD5A1 ZC2HC1A 
CACNA1A EPB41L1 GRM5 LSM11 PANK3 RIMKLB SREBF2 ZFHX4 CACNA1D EXOC6B GRM8 LSS PARP8 RIMS1 SRGAP2B ZNF117 CADM2 EXTL1 HCG18 LYST PCDH11X RIMS2 STARD4 ZNF157 CALN1 FAAH HCN4 MAGI2 PCDH17 RNF144A STAU2 ZNF204P CAMK2G FAIM2 HGNC:18790 MAGI3 PCDH7 RNF152 STK39 ZNF215 CAMK4 FAM110B HGNC:24955 MANEAL PCDHB3 RNF207 STMN2 ZNF334

CCDC71L FAM126B HK2 MAP1A PCLO RNU2-38P STOML1 ZNF441 CCDC88A FAM133A HMGCLL1 MAP3K21 PCYT2 RNU6-1016P STOX2 ZNF443

CD109 FAM155A HMGCR MAP3K9 PDE1A RNU6-130P STRIP2 ZNF514 CD8A FAM169A HMGCS1 MAP9 PDP1 RNU6-137P STXBP1 ZNF555 CDH8 FAM171B HMGXB4 MAPRE2 PGAM1 RNU6-30P SV2A ZNF571 CDKL2 FAM19A2 HOMER2 MAPT PGK1 RPARP-AS1 SV2C ZNF577 CDKL5 FAM216A HOOK1 MBLAC2 PGM2L1 RTN1 SVOP ZNF793 CDR1 FAM228B HPRT1 MDGA2 PGRMC1 RUFY2 SYNE1 ZNF804A CEND1 FAM66C IAH1 MGST2 PI4K2B RUNDC3B SYNPR ZSWIM5 CENPVL1 FASN

\subsection{Attachment 13}

ACTR8 CPNE8 HNRNPC NT5C3A PSMC1 RPL23 RPS25 ST13 AK2 CS HNRNPL NUDC PSMC6 RPL24 RPS6 STK3 ANAPC15 DNAJB6 HSP90AB1 OGFRL1 PSME2 RPL26L1 RPS8 STRADB ANP32B DNAJC8 HSPE1 OLA1 PTGES3 RPL3 RTN4 STX12 ARPC3 DYNC1I2 ISCA1 PABPC1 PXN RPL31 RWDD1 SUMO1 ATP1B3 EEF1B2 KCTD9 PDAP1 RAC1 RPL34 SDHC TANK ATP5E EIF2S2 LPCAT2 PDCL3 RAN RPL35 SET TCP1 ATP5F1 EIF2S3 LYPLA2 PDE8A RAP1B RPL5 SH3BP4 TECR ATP5G2 EIF4H MAP1LC3B PHC1 RBMX2 RPL6 SKP1 TPI1 BAK1 ELL2 MARK1 PIGH RCN1 RPLP0 SMS TTC27 BZW1 FAM45A MBD2 PIK3CB REEP5 RPLP1 SMU1 TUBG1 CACYBP FBXL3 MED26 PIN4 RHEB RPS12 SNRPA1 UBE2M CD58 GALNT18 MFSD9 PKIB RPL13A RPS13 SNRPB2 UBE2S CDC20 GAPDH MRPS10 POLR2J RPL18A RPS15 SNRPF UQCR11 CDC42 GCSH MRPS36 POLRMT RPL19 RPS15A SNX5 WWP1 CDK7 GLRX3 MTRF1L PPP1R15A RPL21 RPS16 SPCS2 YBX1 COX17 GNB4 NDUFA2 PRKAG2 RPL22 RPS2 SSB ZNF706 COX7C HNRNPA1 


\subsection{Attachment 14}

A2M C1orf198 CNN1 FBLN5 LRRC59 PARVA RAB23 STT3A ACOT2 C20orf27 CNN2 FITM2 MATN2 PDLIM7 RASSF2 SULT1E1 ACTA2 C6orf106 COL11A1 GDI2 MGAT1 PFN1 RASSF3 SURF4 ACTG2 CALD1 COL14A1 GFPT2 MID1 PKP2 RCAN1 TAGLN ACTN1 CAV1 COL4A1 GLIPR1 MSRB3 PLD3 RPL22L1 TES ADAM19 CAVIN1 COL4A2 GNPNAT1 MYH9 PLEKHG3 RPS4X TFRC ADPGK CCDC117 COTL1 GOLPH3L MYL12B PLK2 RSU1 TGFB1I1 AHCY CCND3 CPD IER3 MYL9 PMP22 SCRT2 TLN1 AMOTL2 CCZ1 CSRP1 IFITM3 MYOF PODXL SEC31A TMED3 ANXA2 CD24P4 DCBLD1 IL13RA1 NEXN POLR3D SFT2D2 TPM2 APEX2 CDKN2B DDX50 ITGB1 NFKB1 POU4F1 SIX4 TPM4 ARF4 CDR2 DEPDC7 KDELC1 NOMO2 PPM1F SLC38A2 TRAK2 ARID5B CDR2L DHX37 KIRREL1 NOP14 PRPS2 SLC52A2 TRAM2 ARSB CHPF2 DISP1 LATS2 NRTN PTDSS1 SNTB2 TTC9C B3GALNT2 CHRAC1 EIF4EBP1 LIMS1 NXPH4 PUM3 SRM UGDH BCAT1 CKAP4 EIF5A LMAN1 OGFOD1 PXDN SSR3 VASP C12orf45 CLIC4 FAM46B LPP OPA3 PYCR1

\subsection{Attachment 15}

ADO CCT4 FLNA LARS MT-CYB PROX2 SAP30 TOMM34 AEN CHIC2 GAR1 LEO1 MTFR1 PRR7-AS1 SARS TOP3B AGO1 COQ8B GIPC1 LIAS MTHFD1L PTOV1-AS1 SEC14L2 TRMT61A AIMP2 CTDP1 GTF2F2 LIMA1 MYBBP1A PTTG1IP SETD7 TUBD1 AJUBA CTNNAL1 HAX1 LINC00461 NACC2 PUS3 SH3GL1 TWNK ARHGEF2 CTPS2 HDGF LINC00969 NFATC3 PWP2 SHMT2 UBTD1 ARMCX6 DAPK3 HEG1 LINC02060 NFKBIL1 QARS SHROOM3 UBXN2A ATAD3A DDX31 HOXA2 LRWD1 NOB1 RAB9A SMARCC1 UCK2 ATP5G2 DHX34 HS1BP3 M6PR NOL6 RANBP10 SMIM26 UMPS AUTS2 DHX40 HSPBAP1 MAT2B NPM1 RASA4 SPSB4 UROD BOD1 DKC1 HSPH1 MCM7 NUP188 RASL11B SS18 USP25 BVES DPF2 IER5 MED22 ORC5 RBM42 SSNA1 VARS BYSL DPH5 IFRD1 MED27 PDCL3 RCC1 STARD7-AS1 VCL C12orf65 EED IFRD2 METTL12 PHC2 RCC2 SZRD1 VEZF1 C17orf97 EFL1 IGF2BP1 METTL13 PHF23 RGS16 TAF12 WDR46 C19orf53 EIF2S2 ILDR2 MEX3A PIP5K1A RNF114 TCF12 WWC1 C6orf48 EIF3E INTS4 MFSD2A PLEKHA3 RNF182 TMEM104 WWC2 CA14 EIF3L INTS7 MIR181A2HG POC1B RNF41 TMEM161B-AS1 XPO5 CA2 ENAH IPO8 MKRN2 POLRMT RPA2 TMEM187 XRCC1 
CASC10 EPB41L4A-AS1 ITGA4 MOCS3 POP1 RPL7 TMEM200C ZBTB12 CBFB FAM50A JADE3 MRI1 PPM1D RPP25L TMEM206 ZCCHC2 CCDC77 FBL KATNA1 MRPS6 PRELID1 RPS6KB1 TMEM243 ZNF664 CCDC85C FERMT2 KLHL25 MTA2 PROSER1 SAMD4B TNFRSF12A ZNF79

CCNJL

\subsection{Attachment 16}

ACTB CUL3 FGF6 IL3 MAPKAPK5 PRKACA PSMD3 RASGRP4 ACTG1 DAB2IP FGF7 IL3RA MARK3 PRKACB PSMD4 RBX1 ACTN2 DLG4 FGF8 IL5 MOV10 PRKACG PSMD5 RET AGO1 DNAJB1 FGF9 IL5RA MYC PSMA1 PSMD6 RPS27A AGO2 DUSP1 FGFR1 IL6 NCAM1 PSMA2 PSMD7 SEPT7 AGO3 DUSP10 FGFR2 IL6R NCOA3 PSMA3 PSMD8 SHC1 AGO4 DUSP16 FGFR3 IL6ST NEFL PSMA4 PSMD9 SHC2 AKAP9 DUSP2 FGFR4 IQGAP1 NF1 PSMA5 PSME1 SHC3 ANGPT1 DUSP4 FGG IRS1 NRAS PSMA6 PSME2 SOS1 APBB1IP DUSP5 FN1 IRS2 NRG1 PSMA7 PSME3 SPRED1 ARAF DUSP6 FOXO1 ITGA2B NRG2 PSMA8 PSME4 SPRED2 ARRB1 DUSP7 FOXO3 ITGB3 NRG3 PSMB1 PSMF1 SPRED3 ARRB2 DUSP8 FRS2 JAK1 NRG4 PSMB10 PSPN SPTA1 ARTN DUSP9 FRS3 JAK2 NRTN PSMB11 PTK2 SPTAN1 BRAF EGF FYN JAK3 PAK1 PSMB2 PTPN11 SPTB BRAP EGFR GDNF JUN PAK2 PSMB3 PTPRA SPTBN1 BTC ERBB2 GFRA1 KALRN PAK3 PSMB4 RAC1 SPTBN2 CALM1 ERBB3 GFRA2 KBTBD7 PAQR3 PSMB5 RAF1 SPTBN4 CAMK2A ERBB4 GFRA3 KIT PDGFA PSMB6 RAG1 SPTBN5 CAMK2B EREG GFRA4 KITLG PDGFB PSMB7 RAG2 SRC CAMK2D ETV4 GRB2 KL PDGFRA PSMB8 RAP1A SYNGAP1 CAMK2G FGA GRIN1 KLB PDGFRB PSMB9 RAP1B TEK CCND3 FGB GRIN2A KRAS PEA15 PSMC1 RAPGEF2 TLN1 CDC14A FGF1 GRIN2B KSR1 PEBP1 PSMC2 RASA1 TNRC6A CDC14B FGF10 GRIN2C KSR2 PHB PSMC3 RASA2 TNRC6B CDC42 FGF16 GRIN2D LAMTOR2 PPP2CA PSMC4 RASA3 TNRC6C CDC42EP2 FGF17 HBEGF LAMTOR3 PPP2CB PSMC5 RASA4 TYK2 CDC42EP3 FGF18 HRAS LAT PPP2R1A PSMC6 RASAL1 UBA52 CDC42EP5 FGF19 HSPB1 MAP2K1 PPP2R1B PSMD1 RASAL2 UBB CDK1 FGF2 IGF2BP1 MAP2K2 PPP2R5A PSMD10 RASAL3 UBC CNKSR1 FGF20 IL17RD MAP3K11 PPP2R5B PSMD11 RASGEF1A VCL CNKSR2 FGF22 IL2 MAPK1 PPP2R5C PSMD12 RASGRF1 VWF CSF2 FGF23 IL2RA MAPK3 PPP2R5D PSMD13 RASGRF2 WDR83 
CSF2RA FGF3 IL2RB MAPK4 PPP2R5E PSMD14 RASGRP1 XPO1 CSF2RB FGF4 IL2RG MAPK6 PPP5C PSMD2 RASGRP3 YWHAB CSK FGF5

\subsection{Attachment 17}

ADNP ASH1L CUL3 GRIN2B KMT5B POGZ SCN2A SYNGAP1 ANK2 ASXL3 DSCAM KATNAL2 MYT1L PTEN SETD5 TBR1 ARID1B CHD8 DYRK1A KMT2A NAA15 RELN SHANK3 TRIP12

\subsection{Attachment 18}

ANKRD11 CIC ERBIN IRF2BPL MECP2 NRXN1 SLC6A1 TNRC6B BCKDK CNTN4 FOXP1 KAT2B MED13 PHF3 SMARCC2 UBN2 BCL11A CNTNAP2 GABRB3 KDM5B MED13L PTCHD1 SPAST UPF3B CACNA1D CTNND2 GIGYF2 KDM6A MET RANBP17 SRCAP USP7 CACNA1H DDX3X GRIP1 KMT2C MSNP1AS RIMS1 SRSF11 WAC CACNA2D3 DEAF1 ILF2 MAGEL2 NCKAP1 SCN9A TBL1XR1 WDFY3 CHD2 DIP2C INTS6 MBOAT7 NLGN3 SHANK2 TCF20

\subsection{Attachment 19}

ACHE CC2D1A DAPP1 GPHN LMX1B PAH RAB2A STXBP5 ADA CCT4 DENR GRIK2 LZTR1 PARD3B RBFOX1 TAF6 ADCY3 CDC42BPB DIP2A GRIK5 MACROD2 PARK2 ROBO2 TCF7L2 AGAP2 CEP41 DISC1 GRIN1 MBD5 PAX5 SBF1 TERF2 AHDC1 CGNL1 DNMT3A HECW2 MFRP PER2 SCN1A TMLHE AKAP9 CHRNA7 DOCK8 HIVEP3 MTOR PHB SEMA5A TRIO AMPD1 CIB2 DPP10 HMGN1 MYO5A PHF2 SETBP1 TRPC6 AMT CLASP1 DYNC1H1 ITGB3 MYO9B PHRF1 SETD2 TRPM1 ANXA1 CNKSR2 EFR3A KAT6A NAV2 PLCB1 SHANK1 UBE3A APH1A CNOT3 EHMT1 KCNJ10 NINL PLXNA4 SHOX UBE3C ASTN2 CNR1 ELAVL3 KCNQ2 NLGN4X PLXNB1 SLC12A5 UBR5 ATP10A CNTN5 ELP4 KCNQ3 NR3C2 PON1 SLC35B1 USP15 ATP2B2 CNTN6 EP400 KDM5C NRXN3 PREX1 SLC38A10 USP45 AUTS2 CNTNAP4 ETFB KDM6B NUAK1 PRICKLE1 SLC6A3 VIL1 AVPR1A CTCF FAM92B KIAA1586 OPHN1 PRICKLE2 SLC7A3 WWOX 
C11orf30 CTNNB1 FBN1 KIAA2022 OR52M1 PRKCB SLC7A5 ZBTB20 CACNA1E CTTNBP2 FOXP2 KIRREL3 OXTR PRODH SPARCL1 ZC3H4 CACNB2 CUL7 GIGYF1 KMT2E P2RX5 PTK7 STXBP1 ZMYND11 CAPRIN1 CUX1 GPC4 LAMB1 P4HA2 PYHIN1

\subsection{Attachment 20}

ADNP C12orf57 DHCR7 IQSEC2 NSD1 PRODH SIN3A TCF4 ADSL CACNA1C DMD KAT6A NTNG1 PSMD12 SLC1A2 TRIP12 AHDC1 CDKL5 DMPK KMT2A OCRL PTEN SLC45A1 TSC1 AHI1 CHD2 DYRK1A MAGEL2 PACS1 PTPN11 SLC9A6 TSC2 ALDH5A1 CHD7 EHMT1 MBD5 PAX6 RAI1 SMARCA2 UBE3A ALG6 CHD8 EP300 MBOAT7 PCCA RERE SNX14 UNC13A ANKRD11 CHKB FMR1 MECP2 PCCB RNF135 SOX5 UPF3B ARID1B CIC GATM MEF2C PCDH19 RPS6KA3 STAG1 VPS13B ARX CNKSR2 HCN1 NACC1 PIK3R2 SATB2 STXBP1 WAC ASXL3 CNOT3 HDAC4 NF1 POGZ SCN1A SYNE1 YY1 ATP1A3 CNTNAP2 HEPACAM NFIX PPM1D SETD5 SYNGAP1 ZBTB20 BCL11A DDX3X HERC2 NIPBL PPP2R5D SGSH TAF1 ZNF462 BRAF DEAF1 HOXA1 NR2F1 PRKD1 SHANK3

\subsection{Attachment 21}

ACHE CACNA1H DEAF1 GRIN2B MAGEL2 PARD3B RIMS1 STXBP5 ADA CACNA2D3 DENR GRIP1 MBD5 PARK2 ROBO2 SYNGAP1 ADCY3 CACNB2 DHCR7 HCN1 MBOAT7 PAX5 SBF1 TAF1 ADNP CAPRIN1 DIP2A HDAC4 MECP2 PAX6 SCN1A TAF6 ADSL CC2D1A DIP2C HECW2 MED13 PCCA SCN2A TBL1XR1 AGAP2 CCT4 DISC1 HEPACAM MED13L PCCB SCN9A TBR1 AHDC1 CDC42BPB DMD HERC2 MET PCDH19 SEMA5A TCF20 AHI1 CDKL5 DMPK HIVEP3 MFRP PER2 SETBP1 TCF4 AKAP9 CEP41 DNMT3A HMGN1 MSNP1AS PHB SETD2 TCF7L2 ALDH5A1 CGNL1 DOCK8 HOXA1 MTOR PHF2 SETD5 TERF2 ALG6 CHD2 DPP10 ILF2 MYO5A PHF3 SGSH TMLHE AMPD1 CHD7 DSCAM INTS6 MYO9B PHRF1 SHANK1 TNRC6B AMT CHD8 DYNC1H1 IRF2BPL MYT1L PIK3R2 SHANK2 TRIO ANK2 CHKB DYRK1A ITGB3 NAA15 PLCB1 SHANK3 TRIP12 ANKRD11 CHRNA7 EFR3A KAT2B NAV2 PLXNA4 SHOX TRPC6 ANXA1 CIB2 EHMT1 KAT6A NCKAP1 PLXNB1 SLC12A5 TRPM1 APH1A CIC ELAVL3 KATNAL2 NF1 POGZ SLC1A2 TSC1 
ARID1B CLASP1 ELP4 KCNJ10 NFIX PON1 SLC35B1 TSC2

ARX CNKSR2 EP400 KCNQ2 NINL PPM1D SLC38A10 UBE3A ASH1L CNOT3 ERBIN KCNQ3 NIPBL PREX1 SLC45A1 UBE3C ASTN2 CNR1 ETFB KDM5B NLGN3 PRICKLE1 SLC6A1 UBN2 ASXL3 CNTN4 FAM92B KDM5C NLGN4X PRICKLE2 SLC6A3 UBR5 ATP10A CNTN5 FBN1 KDM6A NR3C2 PRKCB SLC7A3 UPF3B ATP1A3 CNTN6 FMR1 KDM6B NRXN1 PRKD1 SLC7A5 USP15 ATP2B2 CNTNAP2 FOXP1 KIAA1586 NRXN3 PRODH SLC9A6 USP45 AUTS2 CNTNAP4 FOXP2 KIAA2022 NSD1 PSMD12 SMARCA2 USP7 AVPR1A CTCF GABRB3 KIRREL3 NUAK1 PTCHD1 SMARCC2 VIL1 BCKDK CTNNB1 GATM KMT2A OCRL PTEN SNX14 VPS13B BCL11A CTNND2 GIGYF1 KMT2C OPHN1 PTK7 SOX5 WAC BRAF CTTNBP2 GIGYF2 KMT2E OR52M1 PYHIN1 SPARCL1 WDFY3 C11orf30 CUL3 GPC4 KMT5B OXTR RAB2A SPAST WWOX C12orf57 CUL7 GPHN LAMB1 P2RX5 RAI1 SRCAP YY1 CACNA1C CUX1 GRIK2 LMX1B P4HA2 RANBP17 SRSF11 ZBTB20 CACNA1D DAPP1 GRIK5 LZTR1 PACS1 RBFOX1 STAG1 ZC3H4 CACNA1E DDX3X GRIN1 MACROD2 PAH RELN STXBP1 ZMYND11

\subsection{Attachment 22}

"zen_code" "ID_pac" "iniciais" "nascimento" "diagnostico" "sexo" "status" "alvo" "exoma" "fra_fr1" "fra_fr2" "fra_fr3" "fra_fr4" "spl_fr1" "spl_fr2" "spl_fr3" "spl_fr4" "sto_fr1" "sto_fr2" "sto_fr3" "sto_fr4" "nfr_fr1" "nfr_fr2" "nfr_fr3" "nfr_fr4" "nsy_fr1" "nsy_fr2" "nsy_fr3" "nsy_fr4" "syn_fr1" "syn_fr2" "syn_fr3" "syn_fr4" "utr5_fr1" "utr5_fr2" "utr5_fr3" "utr5_fr4" "utr3_fr1" "utr3_fr2" "utr3_fr3" "utr3_fr4" "ncRNA_fr1" "ncRNA_fr2" "ncRNA_fr3" "ncRNA_fr4" "miRNA_fr1" "miRNA_fr2" "miRNA_fr3" "miRNA_fr4" "bnd_fr1" "bnd_fr2" "bnd_fr3" "bnd_fr4" "reg_fr1" "reg_fr2" "reg_fr3" "reg_fr4" "lof_fr3" "lof_fr4"

"1" "C11045" 156 "MVMB" 16807 "ELA" "F" "probando" 0208112403783 16480516318317440988144147977116204313214016816457182495 9710515184784113951111407162778682324151327210

"2" "C22147" 157 "JCAO" 21915 "ELA" "M" "probando" 02089536473310 0533121145134029013449495731091739529635139859564195889 10381444718911300011683552748662921561585

"3" "C25973" 158 "GJO" 23485 "ELA" "M" "probando" 020811025361451 653143161840189284985117310915494320271466777110188511288 1115667011373000119736447416431416111447

"4" "C26551" 159 "AMS" 25732 "ELA" "F" "probando" 02076723066340 663162144944487665285477310629457404521343837881833859810 14958112974000114866777658026513101432

"5" "CC1139" 1 "MJR" NA "cfc" "F" "probando" 0688433310037171124 
11741141389565995269510865601321243514235168272139010981301 15543481982253123130205571305134016552649316445

"6" "CC1155" 2 "NSR" NA "noonan" "M" "probando" 068842749855370 48120010351921041644079110773972543839021491382022177733 10741554343126215262021022850927125715855820190408

"7" "CC1158" 3 "NAO" NA "noonan" "M" "probando" 0678424495461 6179018911352880650346086109784943114937882051722421353 1080117815343721712162819300212251211131815752224305395

"8" "CC1162" 4 "LBL" NA "noonan" "M" "probando" 0677103041019115 65813318013442965664655899117536893823943272782472622567 13171262159471424725724240102415216511659175583472325412

"9" "CC1169" 5 "LBS" NA "noonan" "F" "probando" 0690113121008121 70710118310404907668249260110606423534137902551612622064 1309128913841642512262223200218071516138815154435210534

"10" "CC1216" 6 "LAS" NA "noonan" "M" "probando" 0465612596281 5855115183508387509448521015353634246351921117025185411025 105115739631392132715200190471164124813648430293257

"11" "CC1264" 7 "JMLS" NA "noonan" "M" "probando" 04881043293672 66105115613362889858151569108786013624236032321972720612 1290119316443112072453222130203491333135813855839202555

"12" "CC1371" 8 "RHGP" NA "noonan" "F" "probando" 0677820197552 6227417213392889346942194107634202744436341621733121349866 10981594240167210232122021395989117115852835271327

"13" "CC1413" 9 "GFL" NA "noonan" "M" "probando" 069111222106211 2825821927332968451945374116345143534541962131912823064 1092130114946941922662218000240581200155616461037262416

"14" "CC1471" 10 "MCS" NA "noonan" "M" "probando" 06911026410799 06298118910343929668660580115427154433842073482604322275 1473140217045592942562216121242161760178915957053435435

"15" "CC1586" 11 "MAOF" NA "noonan" "M" "probando" 046782338676 06481031331428078206184557793316623564625431911653120546 1197123416139602442472015111147901160106814139129140396

"16" "CC1599" 12 "PVIS" NA "noonan" "M" "probando" 05668324831011 67858215612291834474562785102477994413830372711973221458 15851424180389525925919250101760616311339148443463245112

"17" "CC1980" 13 "DB" NA "noonan" "M" "probando" 066972351201015 376421617812482935966853980112986563794639282472384222315 13171424187462428024923181202274015561641181538362645914

"18" "CC2016" 14 "GPZ" NA "noonan" "M" "probando" 0684428510129 26759116682908872389433102106133382824238471511462120710 73010331744270168220242121021237828117715655627305468

"19" "CC245" 15 "MSGJ" NA "noonan" "M" "probando" 0476422273782 6071031409370789561753989954966037839262623814826205771412 130916439522392512114130147361207108612335333233407 
"20" "CC2492" 16 "WSS" NA "cfc" "M" "probando" 0469733381390757 51154183328347624480741020163538348335722319529186431215 109112839442312232918100186701350126116646545284474

"21" "CC3533" 17 "LFG" NA "noonan" "M" "probando" 06959295121392 7748119611472965943640170117194302605443351782003622730803 116917645241792182529041248901003135816159727311468

"22" "CC3546" 18 "ASF" NA "noonan" "M" "probando" 0663322175663 529101134837176585283816093785112983724751281352919648982 108114336241821831826130135428358379732417154385

"23" "CC3680" 19 "PVACM" NA "noonan" "M" "probando" 06945174107 69261510218353539203498477841145548233134402521417129 2167290212361594343174178222322122556113013601575112727836 8

"24" "CC3682" 20 "LMS" NA "noonan" "F" "probando" 04914161118712 36831541846441931453347292114385333104040232261993521323 994113015444062072122723520227411236139815355437265438

"25" "CC3766" 21 "A" NA "noonan" "F" "probando" 0278621391490607 122146153228535565454911028555534049303019615027203021074 118116939351842032622110165561017107814444825143425

"26" "CC3770" 22 "DFSD" NA "noonan" "F" "probando" 048210282983 111695811678240854954648667105415723322634532241611920189 1066108111441612152131618120193351220119410351238221474

"27" "CC4388" 23 "LVA" NA "noonan" "M" "probando" 0697926210938 161810217811402950974954683116937334574941442972563922576 1497146716346292932532519240241731780168219058047292445

"28" "CC4565" 24 "DNAC" NA "noonan" "M" "probando" 069111276105 970654102186645091006945691181111777137337378225622232 21259145913242024124272280331922021680168315561675364645644 8

"29" "CC4602" 25 "SCSS" NA "cfc" "F" "probando" 06797328121771675 113156164329119676583761112979338737399927823238211241501 1415179428129322628215202244516881578184528342045012

"30" "CC4640" 26 "RPS" NA "noonan" "M" "probando" 04754173111310 16661321728331933859246077115985433344539251991613521423 1076115515844101642242914340225821234135617357838191406

"31" "CC4653" 27 "KSG" NA "noonan" "M" "probando" 04707304104412 57438116212383862250744685104975563385436242221853119533 9321050150399617121427181201983311251289167482342425010

"32" "CC4902" 28 "JMMS" NA "noonan" "M" "probando" 057391901027 716776115814361843271652959105177224183240603072584717463 1292114715340462611962917200209721580146317452838445322

"33" "CC4965" 29 "MMEF" NA "noonan" "M" "probando" 05735264948 121697101144103018676581554901040267340541329522816525 20957121113281884188225231331610019082141113121274733430648 
6

"34" "CC4996" 30 "ASP" NA "noonan" "M" "probando" 06876234111511 163118117012372917368161491113337174195540202642883421659 1405139316944143212793318120225441713163716952738291426

"35" "CC5035" 31 "BMB" NA "noonan" "M" "probando" 0692626510565 06859216374248949424429110108883712383937911591733521276 72610381594342114173321832021091889117918951922186407

"36" "CC5054" 32 "GHOS" NA "noonan" "M" "probando" 0675123741049 706191321561844190169227708411003104661039366841227534 21619192418082144350357272312610020982225519871794815836457 6

"37" "CC5059" 33 "OAFE" NA "cfc" "F" "probando" 0565422292052546 1011589345851939240296100933222526031201271193820436709945 176419312515021233311779969498616046723245375

"38" "CC5064" 34 "SWSS" NA "noonan" "M" "probando" 0557627591107 1721412514811313824679166678100247864783730792752021720359 16911563160401928028830200001762118191558140413532734611

"39" "CC5071" 35 "VSS" NA "noonan" "M" "probando" 06721229310911 103658112182164429024802588831086381041147374630723539 21950153114581584360304278263030021157182315691555065734450 8

"40" "CC5072" 36 "DJOS" NA "noonan" "M" "probando" 0685532510779 2509621685424837358354887104486453754534712801932720508 1217121515341292021992527041196831388137615847523154479

"41" "CC5088" 37 "RSAM" NA "nf1" "M" "probando" 067412266108310 350515018013383884363550291107355773624736012181952821365 1182118615443771581891917521204941236136415851726203519

"42" "CC5097" 38 "RLA" NA "noonan" "M" "probando" 0579727290650 47310115416392896074361681108238454604334192491922521523 1548145918441943112552217410197301682151215352044425423

"43" "CC5098" 39 "ALFC" NA "cfc" "M" "probando" 0594104211161715 3721110115319452921810598156511214112564846362041629140 22219223820052104358437357371631020967247219831975506335367 5

"44" "CC5099" 40 "MVS" NA "noonan" "M" "probando" 04851638511297 16928217517351923558550073114106413794938272511813121551 1212123516544392172112431110209751370140416756133307538

"45" "CC5105" 41 "KMV" NA "nf1" "M" "probando" 0467527398410163 78314715292867457243383105085313294935591561502320034989 113217042191902072722210196531094114213850535259457

"46" "CC5114" 42 "HBF" NA "noonan" "M" "probando" 0576725589462 5710811487352868058853761103065873594231051871592321154 1191127717040632062712717240178671165126014244530193398 "47" "CC5119" 43 "JPMS" NA "noonan" "M" "probando" 0580525595310 
16846117211392885549847683108714883164134021741682822375 1008129517442201722524116230205361066126516356433292417

"48" "CC5120" 44 "ALN" NA "noonan" "M" "probando" 0674528499513 3614721579441884162451673107976523813134732671862521864 1379135614544282242362615120203961456144614251036234489

"49" "CC5133" 45 "APS" NA "noonan" "M" "probando" 0669630395552 7279116612380903358045973109495403633035211721692521882 1014127916543651762422224100202891137141215347638275446

"50" "CC5188" 46 "BHMS" NA "noonan" "M" "probando" 06707315987 112648831717443906452147088108755053443237311982163921795 10441195169440017824123140202142611481388169521182755010

"51" "CC5198" 47 "JLC" NA "noonan" "F" "probando" 0572131293171 61272163153828999536451102107604742854331621631352622002 892115517442761972173224110197861034113616047927203455

"52" "CC5200" 48 "JCS" NA "noonan" "M" "probando" 04681231410114 123561517316230433907211669087411154136974146369245837639 21354256620622054454464409312322020897271422582165427859760 10

"53" "CC5217" 50 "MPS" NA "noonan" "M" "probando" 06761131489411 17221331784413899845640279109154632854435731631211521567 913112616141661602142518110209371021123017652616203558

"54" "CC5219" 51 "AAD" NA "noonan" "M" "probando" 0480919288291 59211217111270867541239183107093882834534731571743520491 80410411814013165191281833119417881112515245323205395

"55" "CC5284" 52 "LFSS" NA "noonan" "M" "probando" 0571425810319 355916616011401872258649887105625853374232981841782521658 11631364158422120622823211301975412541285163481363045017

"56" "CC5299" 54 "ERBS" NA "noonan" "M" "probando" 0575526298751 70417416514380873359449281105165933573632852241943021948 1294131015243372282342622000192871230130414149035304487

"57" "CC5305" 56 "AHS" NA "cfc" "M" "probando" 06621328398710272 8121156194909004802657931068484752643347232123427222171687 156718642042722773420210201641934171215752956316506

"58" "CC5308" 57 "WSS" NA "cfc" "M" "probando" 0683929489971737 71131103748479715558741021570646836325922321735207111447 129515841112532381915120187721526140114547539173436

"59" "CC5399" 58 "MBLF" NA "noonan" "M" "probando" 047462549979 26061531476293858863056188104576714163935112641931919105 1255127416341502782182615210193761427141413547746275499

"60" "CC5407" 59 "GAC" NA "noonan" "M" "probando" 0670325499661 56111316673268765358452101104013302925233491161483520122 6259981774108118193232403019271715111715249218214428

"61" "CC722" 61 "MAM" NA "noonan" "M" "probando" 0479112839235 26471321609320886340538693107003823155335201461473019633 
7889541633975150184231400019342845108416346023221467

"62" "CC726" 62 "MCS" NA "noonan" "F" "probando" 04769312981092 551215516014283817473257681101568004385634872882193118956 1397130718437762152553619210188721567141915643846291559

"63" "CC736" 63 "SMFC" NA "noonan" "F" "probando" 0482827097580 7749016112381920359645739110135202991632061931549232501093 1266644525191219142334019686115412195949834342440

"64" "CC804" 64 "JFCS" NA "noonan" "M" "probando" 0656416065484 51640135523171584104198187843492614128171281403015838685 871137340815414024162101463774294514835212203284

"65" "CC815" 65 "VGS" NA "noonan" "M" "probando" 0662716150582 44392120923166024694157080544922973725971791372814227831 84714733311931602493211313588285910631334189335

"66" "CC883" 67 "VCAB" NA "cfc" "M" "probando" 0470625287341655 631718311836841137889101093512423234961231242218965681930 1903979132192222031019122838105513844122253356

"67" "CC884" 68 "AGN" NA "noonan" "F" "probando" 0670815262231 43693131730166853803607781433482383924611161132713959683 761142293511315529100001299565071212433415153276

"68" "CC893" 69 "AGVC" NA "cfc" "F" "probando" 05831019270463734 9316162818450462386711022945028944306012111126207658231039 1563940140190222102018283911102912344925195348

"69" "CC941" 70 "VMS" NA "noonan" "F" "probando" 0479928481662 6041351708351864366453272107427364463536022532193719758 13461296167424126524920171302025814451465161498322824711

"70" "CC962" 71 "LTSN" NA "noonan" "F" "probando" 05731634597470 54610313517313850279664387103819015214332002652252821361 1724158420740863222802124121189921821156216449552341518

"71" "CC963" 72 "KFFV" NA "cfc" NA "probando" 05851423510246463 1113615983309001529476881080648633445338420616328218761017 1148168430317123231240001999311651248165493182114215

"72" "F-3078-1" 177 "HF" 36741 "TEA" "M" "probando" 11841230337295 492103932252598531750680756331129241701444459776054121041 531028300068803585425520712614911

"73" "F010644-1" 149 "MRC" 40964 "TEA" "M" "probando" 121971028281 61336511122199176079560786731831162381356743214717212622 2873260188191861145160105000172061483151313841943212537

"74" "F010992-1" 150 "ALVT" 40267 "TEA" "M" "probando" 1218713504 965110747205229144509827752653791204573745355208415814022 313722318223194211013576000179711244122414439634163819

"75" "F10291-1" 151 "LIG" 40057 "TEA" "M" "probando" 121835422891 1448342071981049210223407555111121814113344920419189183210 12815929213369177126000177267901192127444132437613

"76" "F10658-1-II" 168 "JPPA" 42573 "zika" "M" "probando" 019621339359 
29155512213493328118570591639825572401381143655871768129 1011314589613182000104267268315927825263606

"77" "F10661-1-I" 160 "LGS" 42234 "zika" "M" "probando" 020719393802 100631226216310351941664469289115316504894012949466152460 1591491816871041291070001265896111049736625226755

"78" "F10691-1" 178 "BSS" 42360 "zika" "M" "probando" 0185611250434 1134210112118283036705874763798011951536437931006241154 16311511105310812010100074889878596821030151475

"79" "F10692-1" 179 "MAC" 42163 "zika" "F" "probando" 0184810354452 705151929413272652372270272800473552536792927891127139104 1011301011117210074808567666721527150616

"80" "F10693-1-II" 161 "MAC" 42302 "zika" "F" "probando" 020861240278 770657821539465953368472195113976695053112451065342617189 156231793113159790001277596210658738227164554

"81" "F10701-1-I" 162 "MOSL" 42373 "zika" "F" "probando" 02079925284 31655381441311336289626496678810733654463431071845752450 186133131530108123950001169583090877338232015511

"82" "F10741-1-I" 163 "PGS" 42330 "zika" "M" "probando" 0208712364684 10372112031488352930855257983109456064322612167282102427 1461341516169612312500012282858100788315181526610

"83" "F10747-1-I" 164 "RNLCCB" 42371 "zika" "F" "probando" 020748485 945100634120163936193896467368611231674493491178867612 26142021711818151301466511012767101610968637325151705

"84" "F10760-1" 152 "SEO" 41269 "TEA" "M" "probando" 121807374917 1647271442131943298557587439311710813549332018190127192907 2672122419301371621250001694614431451113420342416712

"85" "F10763-1-I" 169 "ITE" 42007 "zika" "F" "probando" 01910033937857 078715421214387102165966501071208857845857185911494103346 19418322213714418498010174391066128412545135253617

"86" "F10777-1" 170 "GSI" 41519 "TEA" "M" "probando" 1198785129068 265623320714473101416566439412240678429491888130100102862 20815019195012915267000163901129119810541733271827

"87" "F10788-1-I" 171 "JLSA" 42236 "zika" "M" "probando" 019766363714 10066121731581329486796467039710554639492411294847691881 14713522156910413846000113908669628931226253636

"88" "F10789-1-I" 172 "HBG" 42474 "zika" "F" "probando" 019657355603 1035981311351632181356586488599747344845510839562121594 1419912133611714954000100727868537926519185589

"89" "F10798-1" 173 "NPC" 41657 "TEA" "F" "probando" 1198844517217 180512418612464997338754593118633683274017526174172725107 116111871731251120001515058891410239212221646

"90" "F10865-1" 165 "IVSN" 39455 "desconhecido" "F" "probando" 020899 41362313363518315614377903758963384107095633884311818160 10219712111515155010610363021115797578988730018223729 
"91" "F10885-1" 154 "GBI" 38803 "TEA" "M" "probando" 121819406782 100638154211636394854165178411573397324431921856615269387 148211825571036900016361699971111389221556510

"92" "F10980-1" 155 "DMAS" 40402 "TEA" "M" "probando" 12173538381 810177517120018384925058357068112415634374618079282132546 14715623169597125830001520581499912135627142655

"93" "F1385-1" 73 "MBA" NA "TEA" "M" "probando" 178353139479375 612716394218879598588771078556038843358119718023203921072 1139162434624029631193202030112021360129550372025213

"94" "F1389-1" 74 "MBG" NA "TEA" "F" "probando" 177713341080122 63313516712311847937042280101963732224034421371603819415 61591812541861242682324120190137641098148447222165811

"95" "F1665-1" 76 "IRS" 34761 "TEA" "M" "probando" 179033331282111 7897119912452976160751169118755573664244572362664622086 1229140815744902283022826230225791272148115452621272515

"96" "F1697-1" 77 "LMF" 36390 "TEA" "M" "probando" 17705343917136 6211121584303863939139176104223282474434941311233119699647 853127417211525531241201994080396714951292345811

"97" "F1722-1" 78 "AR" 32549 "TEA" "M" "probando" 179333429868381 61251747251907842342381109893482713936901501574220968722 101416443201362532827120214798711175183537142235410

"98" "F1729-1" 79 "GSS" 36989 "TEA" "F" "probando" 176510252106295 57511215911333861974962895104827834994435122892202719993 1569141016146133343332624210202101769163015146148246459

"99" "F1903-1" 80 "LMC" 38023 "craniossinostose" "F" "probando" 0660621 3666834561021207332734939938384882736927848274614013323 16455813865142353113114618140001498173993513638618234398

"100" "F2452-1" 81 "IM" NA "TEA" "F" "probando" 1776931572412666 71131531140381064454426897944242913233331741432718498792 92812539691982851921020177398941017129475241425414

"101" "F2570-1" 82 "MMCS" 27933 "CLP" "F" "probando" 037352011097 174665631851248394237186041021157273943139410931825733 22597154214891944608262248252420024376172717351885804928643 8

"102" "F2613-1" 83 "IPCC" 36240 "TEA" "M" "probando" 1374740610155 37231211458381866440645064104814112783534141581542719602 74691714542911582621524122191988311083120474172065710

"103" "F2685-1" 84 "EHK" 37519 "TEA" "M" "probando" 1118141561063 1026947416182639034339542108109753833546240071552045018830 66696515739751572172520021160846431015157364151913212

"104" "F2688-1" 85 "CAMC" 37482 "TEA" "M" "probando" 176792327164 2487411598381789852143177962048727638272518614424199181099 112617738721831912316120159201061105814941220212315

"105" "F2735-1" 86 "LTC" 35529 "TEA" "M" "probando" 1772425482462 
60971155634478464144069694143812614726791291122419770769 988192365114613224141201531176796715637424195387

"106" "F2848-1" 87 "HEN" 39284 "CLP" "M" "probando" 0115983106934 158691121925171954674787888555152983729801741482414306907 8701193253146220229100118177657729625819122442

"107" "F3104-1" 88 "LPM" 38964 "FG" "M" "probando" 01485637581663 5581621557280876954363073106135504164841941952043817064914 90715036201812502316011153558411107139322231725910

"108" "F3196-1" 89 "JCS" NA "CLP" "M" "probando" 047210284934102 603142165123808300564454108102445803064335232101943419002 1032113816840462262422214110196811248134716153123202528

"109" "F4442-1" 90 "JVM" 39209 "CLPSy" "F" "probando" 016513459446 16258115513293868955051478105645643383435152001713019800 1105117614440541901801920100179851133120115844926265487

"110" "F4482-1" 91 "VSS" 38249 "TEA" "M" "probando" 13838375103541 6778217114492903763460677109586344475137572201813121003 1312114312644932383482621220207051422142016051644356498

"111" "F4483-1" 92 "BOJ" 39807 "Saethre-Chotzen" "F" "probando" 0272421 2795805691201357294741462349087909166637736270922315226 17835130712971393670243225181406314643124810811284063722241 2

"112" "F4485-1" 93 "DNS" 31131 "acrofrontofacionasal" "F" "probando" 01 607220514615237281102625740471431576948470332301754148 1211112703931938142291418916313151109429720748722762322335 3

"113" "F5389-1" 94 "KDS" 36860 "frontonasal" "F" "probando" 066816280 10397262460170144339027745619751097577846741384431222232 20967157913891784256272250291211021128172215691725114330741 2

"114" "F5414-1" 95 "VEFA" 39792 "OAV" "M" "probando" 066872438479 44061321351448370004914187089694512942939962352363413262 7927591173693181241248000223451291155716059736240469

"115" "F5502-1" 96 "JPP" 38373 "TEA" "M" "probando" 11182629697240 6468114182929054600562891107056536541398324320940194921069 115114539621872161718242165091027102813838822151417

"116" "F5547-1" 97 "BCP" 39389 "TEA" "F" "probando" 11184629298511 46161871389332902242143985109624482543240341551563619276 6949021513909151184281813016688720880151377141535813

"117" "F5576-1" 98 "LCSS" 38549 "TEA" "F" "probando" 1379726199610 164717418116384919849752788111394473534937231671682221084 914101614743912113211820130206481109124713249533164536

"118" "F5577-3" 99 "MSP" 25809 "auriculo_condilar" "M" "probando" 01365 73236915149711410941817438453417679292461335283290173143 22138017146871062992162169181222011237600649862111381488 
"119" "F5616-1" 100 "VCB" 38964 "TEA" "M" "probando" 1784528590511 16227015911321852844848077103233813063635691851702418999 7559841484063159297262013118522879113813444828182466

"120" "F5674-1" 101 "VBG" 36352 "TEA" "M" "probando" 1776524690414 167511116793338803404455108106593342554636331411674320229 7209581634320133243212323019471827110415347417183498

"121" "F5979-1" 102 "LDO" 38552 "TEA" "M" "probando" 1777825399211 16281441869455873749053793109014583504136652082144120351 952112313442791872601822220202421065132717249917292508

"122" "F5980-1" 103 "JNS" 38137 "TEA" "M" "probando" 1965122311095 153525111169143548347691536641018174542242448435228337 19971134614481604041264301361501023095182717961875275623249 5

"123" "F5996-1" 104 "TSS" 38480 "TEA" "F" "probando" 1771542572781 65101521539382827254354694100565513744232682081954218789 1093115116140461952583018220179121175126614942131244658

"124" "F6021-1" 105 "CBA" 37713 "TEA" "M" "probando" 195762847447 14131021321132262655064198278894642803434482311721813371 83185213028671612031514120165861080113413237222142457

"125" "F6025-1" 106 "JGNG" 27939 "DE" "M" "probando" 0773928784138 359714516564938903531416104109224843086238902091744220550 9351056175424415218922143302085211331251224481172875015

"126" "F6034-1" 107 "BMK" 37859 "TEA" "M" "probando" 196281527034 3343621341627260773793684175583622553133831961543212769757 811106286213118121911016509985106612737623173257

"127" "F6083-1" 108 "ASO" 38495 "oculoauriculofrontonasal" "F" "probando" 066423558967172370159929184885105158710344507313423483 205191382006410171011135409918922741201201984112011268153 49531265496

"128" "F6123-1" 109 "VCB" 38857 "TEA" "F" "probando" 1959616767761 45262129725161984133795876424102582534421931884313155777 85413329261351951916200164449521129137346161612810

"129" "F6131-1" 110 "GSC" 38501 "TEA" "M" "probando" 195832147246 1326113131731062395544737478285903493533952682343613474 109493214130001922372921200161171270125116036529206388

"131" "F6163-1" 112 "GTSM" 39185 "TEA" "F" "probando" 19503172683 152436113131926261214775148976195023773733832202163913133 950103316631001622712914020155831029134818433919285437

"132" "F6167-1" 113 "IAN" 39353 "TEA" "F" "probando" 1963102127139 3323121123731660884544576476144883184432272271874412908902 89712329291632122113200154661034111812833921153426

"133" "F617-19" 114 NA NA "CLP" NA "probando" 0257617176741543 8211212311757351841173904142729449266615313829178129251016 16835301931252216142136348498271043172580294 
"134" "F6281-1" 115 "RGM" 36802 "TEA" "M" "probando" 178252489529 0694611719311896654647579107155273344138362201532420838990 114514943481842062322220213551184135714750333275399

"135" "F6325-1" 116 "MRNS" 39099 "TEA" "M" "probando" 1119211180 105691719165148143649311667589741131174642047419425721327 19767125210821264043214254242152017247118811061244152925343 6

"136" "F6331-1" 117 "LAF" 38395 "TEA" "F" "probando" 1484433484692 5178417192938831641502711078766036236388125119336205671195 1270185443224525530190202131714741455173531443775010

"137" "F6366-1" 118 "PCO" 39333 "TEA" "M" "probando" 196062228549 2459601281123164504444665979304673293534362182083514033855 90312330481542262211140168371052121314835724276374

"138" "F6594-1" 119 "IBB" 35182 "quebra_Ina" "F" "probando" 0387530385 106055491157103738320636540791019470636230344926118528 20180141512271274196272259231631019115142913851284504330345 4

"139" "F6642-1" 120 "PRV" 39545 "TEA" "M" "probando" 196052037075 047440114729361873913865475603832444432671911643613037755 8081262850124226271413116171854104014136118243293

"140" "F6645-1" 121 "VMA" 37145 "TEA" "M" "probando" 187310210913 115516811655373838148050189101584073233234131581892019752 84410451484116184284202321019733965120514047224275406

"141" "F7511-1" 122 "VHFPS" 38343 "TEA" "M" "probando" 17591135289 36154312214315363775254556187932056934343308519818028 18766105111161573605217319321502016866117512601634063621553 5

"142" "F7614-1" 123 "AOLB" 40471 "CLP" "M" "probando" 03727154963 915751631618322833350442891101314853004034841791772719073 100496815340262362232118110186031119101014943930231408

"143" "F7935-1" 124 "JGCE" 40060 "TEA" "F" "probando" 137710347109 121746011184155243238931102079479109241062613463835383294 36213812040169516943994163903620020210882290193218155372413 6915

"144" "F7936-1" 125 "CESS" 37677 "TEA" "M" "probando" 147982231037 1016677117313375885380761672108017734634936523222373620528 1570143418642772582211914130212071775154716352146261395

"145" "F8069-1" 126 "DHF" 34976 "SMD Cone Rod Dystrophy" "M" "probando" 01771022495960694112158835690357036018210962744 47940323228821244218541637150417141142912332422210177741508 135215543239303396

"146" "F8118-1" 127 "HOGBS" 34173 "TEA" "M" "probando" 1477725688 250603811604311828142637271102273752413035211481482919678 7269601644140152190291911020322875104714150317233387 
"147" "F8123-1" 128 "LSS" NA "SMD Cone Rod Dystrophy" "F" "probando" 01889292111860665711751827194397135806411295767450323618 274225392165114881442154448929422918130101900315341428158 47540204423

"148" "F8124-1" 129 "TN" 37765 "SMD Cone Rod Dystrophy" "F" "probando" 01791022511296175871153183809342753604651134079147431 3722301218242237315521553137444228326014224201971815891554 13548433322357

"149" "F8128-1" 130 "IRS" 41141 "condrodisplasia_punctata" "F" "probando" 0769103359887570415116494138412547480789970575342403302 209169331994911301172160415924421122184201781911541159146 411272055511

"150" "F8368-1" 133 "MHR" 41014 "craniossinostose" "M" "probando" 01282 62427958365816420314334932250848110011616489327541736102 7411286013515733138380901260001467573888811236117192489

"151" "F8382-1" 134 "AJA" 41102 "craniossinostose" "F" "probando" 012101 15302853927531521911844397066475657111779681383482048151 121133458220187101606103101128010169181169121511241828202 546

"152" "F8385-1" 135 "TGS" 36189 "Sener" "F" "probando" 067992339456 16369115812383842187965379103889135453836413552363219809 1831153217341713112761919230201502008179215649859392385

"153" "F8402-1" 136 "IZSS" 39910 "TEA" "M" "probando" 1373925310989 06911032039427948251359596114045503584040551792023522176 1042120418248972013773420220231431308135919154645302446

"154" "F8442-1" 137 "VFP" 33870 "TEA" "M" "probando" 1484426499189 0609143153153548619957757102104589725964936613662823320173 1916158816841513482712821230206502159183116750661462497

"155" "F8474-1" 138 "GAO" 34001 "DE" "M" "probando" 0660430591512 36471021415382840246838968101004423094832811481493519267 81399915240401772003018031186788741144164531241635210

"156" "F8552-1" 139 "TPSP" 41096 "craniossinostose" "M" "probando" 01464 5335823935231761391237384255186369910067517383453923197 2053516942858956146338019626834192211425380410671223301523 25914

"157" "F8613-1" 140 "MBG" 31811 "OFD" "F" "probando" 0973132841033 527141651869331824548946372101184482924444362332434519813 8911138140390418827432140102288611901523183506262414911

"158" "F8807-1" 142 "MAUN" 40471 "Rolland-Desbuquois" "F" "probando" 0 96572551005737181431731030485434884858710105459371534482 17424447193639691228178380616424534182212224410601630219479 273844611

"159" "F8823-1" 175 "COPJ" 39564 "TEA" "M" "probando" 119742355601 91583153142931281704465076810164404301341154756219174896 
641313415610494000104135546949727117107599

"160" "F8864-1" 180 "RN2SVA" NA "espondilocostal" "M" "probando" 018 53132854621045711111091816397549548667991500435349086061 911898469121098841272200080886587355720414103406

"161" "F8959-1" 143 "KMTF" 39712 "noonan" "F" "probando" 098511223 10699049710117916534814072066354971677648119433133830933 19372150614061054169308329202201023355197219951485125344541 4

"162" "F8978-1" 144 "STFS" 40514 "displasia espondilo-epifisária" "F" "probando" 015715372763915110192119113747971613671609803 5224774635831982043915275101610101383416184327261502012831 888116010625920181655

"163" "F8982-1" 145 "DMFC" 36259 "noonan" "M" "probando" 099015253 100510251210016112360811447944234992244228514434120024219 19180858114958381916124172633022466112515509245030180455

"164" "F9187-1" 166 "AYHC" 40982 "craniossinostose" "M" "probando" 020 91105298561517151931788453981349465812111584461449591385 63691126741171462318887914497000135986519981233561715186 13

"165" "F9320-1" 146 "DACL" 35042 "craniossinostose" "F" "probando" 014 56621675511247415410182427550385532799561344304333685161 167341411257464111031571342071715010125725897659926111134 4712

"166" "F9470-1" 147 "MNS" 39025 "craniossinostose" "F" "probando" 01577 9575915131642222149935189766677628410913604486363910236 21028182341111122716838942023612520130158581070146815734828 130928

"167" "F9485-4" 176 "JFS" 24898 "mao_torta" "M" "probando" 0191016292 93512177914023818443101278787498412215815567381826178112 19307027519814216014519876000170831470141210645229233553

"168" "F9893-1" 148 "CECS" 41879 "DE" "M" "probando" 0157574738069 2521023311814324807760073962100205764823236221971972916293 99710321213468263354321631013072898119710727324151798

\subsubsection{Prefilter}

\#!bin/bash

\# este script irá filtrar o vcf a partir de um arquivo bed e irá preparar o arquivo para ir no $\mathrm{R}$

\# Versão 1: esta versão é útil apenas para 179 indivíduos, fora isso tem que 
mudar o script

\section{MENSAGEM_HELP="}

Uso: \$(basename "\$0") [OPÇÕES]

OPÇÕES:

-a vcf original (obrigatório)

- $b$ arquivo bed dos genes

-h Mostra esta tela de ajuda e sai

-V Mostra a versão do programa e sai

"

while test -n "\$1"

do

case "\$1" in

-a)

shift

$\mathrm{Vcf}=" \$ 1 "$

i;

$-b)$

shift

bed $=" \$ 1 "$

;i

$-h)$

echo "\$MENSAGEM_HELP"

exit 0

;i

$-\mathrm{V})$

echo $\$$ (basename "\$0")

grep "^\# Versão" "\$0" | tail -1 | cut -d : -f1 | tr -d \\# exit 0

;i

esac

shift

done

bgzip $\$ \mathrm{Vcf}$

tabix - p vcf $\$$ Vcf.gz

vcftools --gzvcf \$Vcf.gz --bed \$bed --out \$bed.output --recode --keepINFO-all

perl / usr / local/annovar/ convert2annovar.pl -format vcf4old \$bed.output.recode.vcf -includeinfo -outfile \$bed.avinput 
perl / usr/local/annovar/table_annovar.pl \$bed.avinput / usr/local/annovar/humandb/ -protocol refGene,exac03,kaviar_20150923,hrcr1,esp6500siv2_all,1000g2015aug_all,gno mad_exome,60mais609_AC,60mais609_AN,snp135,avsnp147,clinvar_2016030 2,dbnsfp30a,dbnsfp31a_interpro,dbscsnv11 -operation g,f,f,f,f,f,f,f,f,f,f,f,f,f,f,f,f,f -

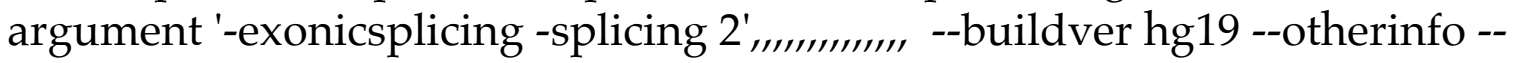
remove -nastring NA -outfile \$bed.2017

perl / usr/local/bin/IncluiColunasInfo2017mar.pl \$bed.2017

bash / home/saturno/ngs/alunos/tfalmeida/scripts / prep.sh -a \$bed.2017.hg19_multiannoFINALREG2017.txt -b 60mais609_AC -c 60mais609_AN

zgrep $\wedge$ \#CHROM \$Vcf.gz > headpac.txt

head -1 \$bed.2017.hg19_multiannoFINALREG2017.txt.filtro.txt > headann.txt

cut -f1-99 headann.txt > header1.txt

cut $-\mathrm{f} 100-102$ headann.txt $>$ header2.txt

cut -f1-7 headpac.txt $>$ header3.txt

cut -f8-188 headpac.txt > header4.txt

paste header1.txt header3.txt header2.txt header4.txt $>$ header5.txt

sed -e 's / ACMG /Exclusion/XACMG/g' header5.txt I sed -e 's / esp6500siv2_all/Freq1/g' I sed -e 's/1000g2015aug_all/Freq2/g' I sed -e 's/60mais609_AF/Freq3/g' > headp.txt

cat headp.txt \$bed.2017.hg19_multiannoFINALREG2017.txt.filtro.txt I sed '2d' > \$bed.anotado.txt

cut -f1-7,9,19,42,43,55,107-110,112-290 \$bed.anotado.txt > \$bed.anotado.cut.txt

rm \$bed.avinput \$bed.2017. ${ }^{\text {head }}{ }^{*}$

\subsubsection{Spring}




\section{Classificação das variantes}

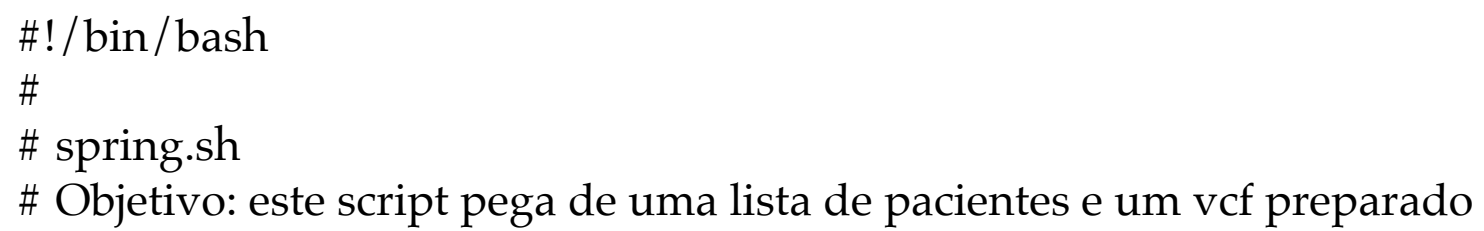
as variantes não-sinônimas para colocar no SPRING

\#

$\#$

\# Autor: Tatiana Almeida

$\#$

\# Versão 1: a partir de um vcf preparado este script filtra as variantes não sinônimas de qualidade e frequencia descritas para utilizar no SPRING para cada paciente

\section{MENSAGEM_HELP="}

Uso: \$(basename "\$0") [OPÇÕES]

\section{OPÇÕES:}

-a vcf anotado (obrigatório)

-b lista das identificações dos pacientes em uma coluna, sem header

-c última coluna antes de começar os pacientes (obrigatório, se colocado os pacientes)

-d dá um valor para filtrar a probabilidade genotípica, se não quiser filtrar nenhum coloque 0 (obrigatório)

-e filtro do $\mathrm{VAF}$, se quiser manter as variantes acima de $30 \%$ de proporção o VAF a ser colocado é 0,6 . Se não quiser filtrar colocar 0.

-f Nome da coluna com 6500exomas

-g Nome da coluna com 1000Genomas

-i Nome da coluna de frequencia alélica da amostras (^AF)

- j Nome da coluna com frequencia alélica dos velhinhos

$-\mathrm{k}$ frequencia de variante benigna

-1 frequencia de polimorfismo

-m Nome da coluna com contagem alélica da amostra (^AC)

-n Número de variantes privativas para o modelo (indivíduo $=1$ )

-o escolher a frequencia em que vai calcular as variantes (benigna, poli, rara, privativa)

-p arquivo ou OMIM para o seed do SPRING

-h Mostra esta tela de ajuda e sai

-V Mostra a versão do programa e sai 
while test $-n$ "\$1"

do case " $\$ 1 "$ in

-a)

shift

$\operatorname{anotVcf="\$ 1"~}$

if test -z "\$anotVcf"

then

echo "Faltou vcf anotado"

exit 1

fi

;i

$-b)$

shift

listapac $=" \$ 1 "$

if test -z "\$listapac"

then

echo "Faltou coluna do paciente" exit 1

fi

i”

-c)

shift

ultimo="\$1"

;i

$-d)$

shift

filter="\$1"

i;

-e)

shift

vaf $="$ " $1 "$

i;

-f)

shift

evs6500="\$1"

;i

$-g)$

shift

MilG="\$1"

;i

-i) 


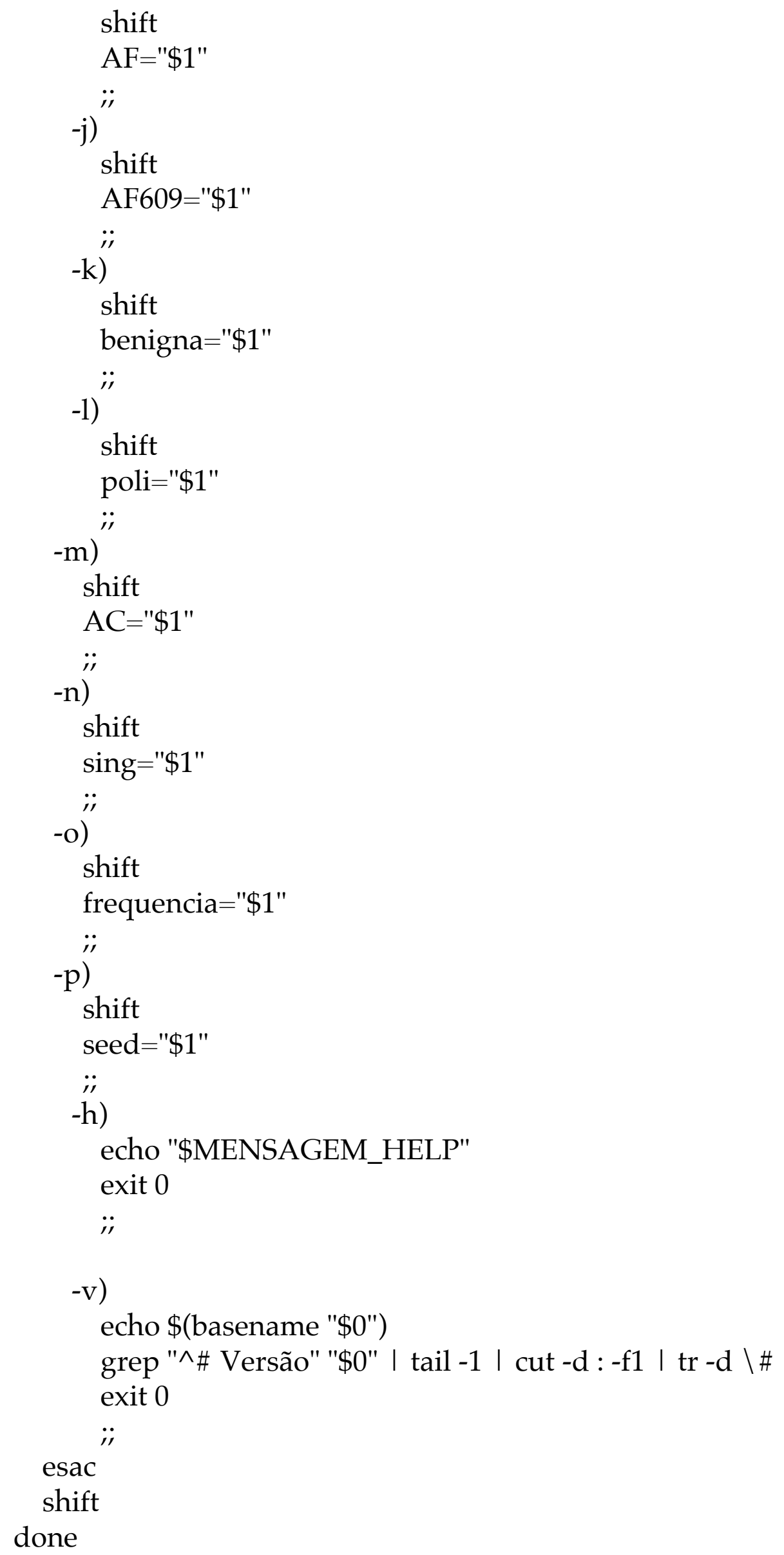


head -1 "\$anotVcf" I sed 's/ \./_/g' > header

numultimo $=\$($ awk $-\mathrm{F} \backslash \quad$ ' $\{$ for $(\mathrm{i}=1 ; \mathrm{i}<=\mathrm{NF} ; \mathrm{i}++)\{\mathrm{if}(\$ \mathrm{i} \sim /$ '\$ultimo' $/)\{$ print

i $\}\}$ ' header)

num6500 $=\$($ awk $-\mathrm{F} \backslash \quad$ ' for $(\mathrm{i}=1 ; \mathrm{i}<=\mathrm{NF} ; \mathrm{i}++)\{\mathrm{if}(\$ \mathrm{i} \sim /$ '\$evs6500' $/)$ \{print i $\}\}\} '$

header)

numMilG=\$(awk $-\mathrm{F} \backslash$ ' $\left\{\right.$ for $(\mathrm{i}=1 ; \mathrm{i}<=\mathrm{NF} ; \mathrm{i}++)\left\{\mathrm{if}\left(\$ \mathrm{i} \sim / \mathrm{IMilG}^{\prime} /\right)\right.$ \{print $\left.\left.\left.\mathrm{i}\right\}\right\}\right\}^{\prime}$ header)

numAF $=\$\left(\right.$ awk $-\mathrm{F} \backslash \quad$ ' $\left\{\right.$ for $(\mathrm{i}=1 ; \mathrm{i}<=\mathrm{NF} ; \mathrm{i}++)\left\{\mathrm{if}\left(\$ \mathrm{i} \sim / \mathrm{AFF}^{\prime} /\right)\{\right.$ print $\left.\left.\mathrm{i}\}\right\}\right\} '$

header)

num609=\$(awk -F $\backslash \quad$ ' for $(\mathrm{i}=1 ; \mathrm{i}<=\mathrm{NF} ; \mathrm{i}++)\{\mathrm{if}(\$ \mathrm{i} \sim /$ / $\$ \mathrm{AF} 609$ ' / ) \{print $\mathrm{i}\}\}\} '$

header)

num $A C=\$\left(\right.$ awk $-\mathrm{F} \backslash \quad$ ' for $(\mathrm{i}=1 ; \mathrm{i}<=\mathrm{NF} ; \mathrm{i}++)\left\{\mathrm{if}\left(\$ \mathrm{i} \sim / \mathrm{IAC}^{\prime} /\right)\right.$ \{print $\left.\left.\left.\mathrm{i}\right\}\right\}\right\}$ ' header)

echo "O núemro da última coluna antes dos pacientes é: \$numultimo"

echo "O número da coluna do 6500 é: \$num6500"

echo "O número da coluna do 1000Genomas é: \$numMilG"

echo "O número da coluna do AF é: \$numAF"

echo "O número da coluna do AF609 é: \$num609"

echo "O número da coluna do AC é: \$numAC"

echo "O valor do benigno do benigno é: \$benigna"

echo "O valor do benigno do polimorfismo é: \$poli"

echo "O valor da variante privativa é: \$sing"

echo "O valor do filtro de GL é: \$filter"

echo "O valor do filtro de VAF é: \$vaf"

echo "As frequencias feitas são: \$frequencia \# ainda não funciona"

\# Retirando apenas as variantes não-sinônimas

grep -w nonsynonymous \$anotVcf $>$ \$anotVcf.nsy

\# Retirando apenas as variantes benignas

awk -F \'\$num6500' >= '\$benigna' | | \$'\$numMilG' >= '\$benigna' I | \$'\$num609' >= '\$benigna' \{print \$0\}' \$anotVcf.nsy > \$anotVcf.nsy.benigna

\# Retirando as variantes entre benigna e polimorfismo

awk -F \BEGIN\{OFS=" ";\}\$'\$num6500' < '\$benigna' \&\& \$'\$numMilG' < '\$benigna' \&\& \$'\$num609' < '\$benigna' \{print \$0\}' \$anotVcf.nsy | awk -F '\$'\$num6500' >= '\$poli' | | \$'\$numMilG' >= '\$poli' I | \$'\$num609' >= '\$poli' \{print \$0\}' > \$anotVcf.nsy.poli 
\# Retirando as variantes raras

awk -F \ '\$'\$num6500' < '\$poli' \&\& \$'\$numMilG' < '\$poli' \& \& \$'\$num609' $<$ '\$poli' \{print \$0\}' \$anotVcf.nsy > \$anotVcf.nsy.rara

\# Retirando as variantes privativas

\# preciso melhorar essa conta porque pode ter algumas frequencias que não trazem valor, ou é NA. Checar isso.

awk -F \'\$'\$numAC'== '\$sing' \{print \$0\}' \$anotVcf.nsy > \$anotVcf.nsy.privativa

\# Criando arquivos SPRING por indivíduo e frequencia

for colpac in $\$$ (cat \$listapac)

do echo \$colpac header) numpac $=\$($ awk $-\mathrm{F} \backslash$ ' $\{$ for $(\mathrm{i}=1 ; \mathrm{i}<=\mathrm{NF} ; \mathrm{i}++)\{\mathrm{if}(\$ \mathrm{i} \sim /$ ' $\$$ colpac' $/)$ \{print $\mathrm{i}\}\}\}$ echo "\$numpac" awk -F\ '\$'\$numpac' ! "0/0। \./\\." \{print \$0\}' \$anotVcf.nsy.\$frequencia I sort I uniq > \$colpac.tmp.txt awk -F $\backslash$ 'BEGIN\{OFS=" "; \}\{split(\$'\$numpac',a,":"); if (a[4] > '\$filter') print \$0\}' \$colpac.tmp.txt > \$colpac.filter.txt

rm \$colpac.tmp.txt awk -F $\backslash$ 'BEGIN\{OFS="";\}\{split(\$'\$numpac',a,":"); split(a[2],b,","); gsub("\\.","0",b[1]); if ( b[1] != 0 ) n = b[2]/b[1]; else $n=0$; if (n > '\$vaf') print $\$ 0\} '$ \$colpac.filter.txt > \$colpac.nsy.\$frequencia.txt rm \$colpac.filter.txt done

\# Cortando o arquivo para ser compatível com o SPRING

\# Cromossomo Posição Ref Alt

for colpac in \$(cat \$listapac)

do cut -f1,2,4,5 \$colpac.nsy.\$frequencia.txt I sed -e 's $/ \wedge \mathrm{chr} / / \mathrm{g}$ ' > \$colpac.\$frequencia.spring.txt done \# Rodando o SPRING 
for colpac in $\$$ (cat \$listapac)

do

echo \$colpac

/ home/ saturno/ngs/alunos/tfalmeida/scripts/spring -p -o

\$colpac.result -s \$seed \$colpac.\$frequencia.spring.txt

done

\title{
Escolha do rank 1
}

lista $=" \$ 1 "$

for pac in $\$$ (cat \$lista); do cut -f5-9 \$pac.result | awk -F $\backslash$ 'BEGIN $\{\mathrm{OFS}="$ ";\} \$3 == 1 \{print "'\$pac"',\$0\}' >> rank1_\$lista; done

\subsubsection{Counting}

\author{
library(dplyr) \\ library(magrittr) \\ library(tidyr)
}

\#\#\# funções

count $<-$ function $(\mathrm{df}$,fr1,fr2,pac $)\{$

soma $<-\operatorname{matrix}($ ncol $=5$, nrow $=\operatorname{nrow}($ pac $))$

for $(\mathrm{j}$ in 1:nrow(pac) $)\{$

as.character $(\operatorname{pac}[\mathrm{j}, 1])->\mathrm{n}$

df[is.na(df)] <- 0

tidyr::separate_(df, n, into = c("GT", "AD","DP","GQ","PL"), sep = ":") -> a a <- dplyr::filter(a, (GT =="0/1" | GT =="1/1") \& as.numeric(DP) > 10 \& as.numeric $(\mathrm{GQ})==99)$

tidyr::separate $(\mathrm{a}, \mathrm{AD}$, into = c("AD1", "AD2"), sep = ",") $->$ a

a $<$ - dplyr::filter(a, as.numeric(AD2)/ as.numeric(DP) >0.3)

$\mathrm{i}=2$

frq1 <- dplyr::filter(a, as.numeric(Freq3) $>=$ fr1 $\mid$ as.numeric(Freq1) $>=$

fr1 $\mid$ as.numeric(Freq2) $>=$ fr1)

frq2 <- dplyr::filter(a, (as.numeric(Freq3) < fr1 \& as.numeric(Freq1) $<$ fr1 $\&$ as.numeric $($ Freq2) $<$ fr1 $) \&$ (as.numeric(Freq3) $>=$ fr2 $\mid$ as.numeric(Freq1) $>=\mathrm{fr} 2 \mid$ as.numeric(Freq2) $>=\mathrm{fr} 2)$ )

frq3 $<-$ dplyr::filter(a, as.numeric(Freq3) $<$ fr2 \& as.numeric(Freq1) $<$ fr2 $\&$ as.numeric $($ Freq2 $)<$ fr2)

frq4 $<-$ dplyr::filter(a, grepl("^AC=1;", INFO) \& as.numeric(Freq3) $<=0$ $\&$ as.numeric $($ Freq2) $<=0 \&$ as.numeric $($ Freq1 $)<=0$ ) 


$$
\begin{aligned}
& \mathrm{n}[\mathrm{i}]<- \text { dplyr::filter(frq1, GT == "0/1") } \%>\% \operatorname{nrow}() \\
& \mathrm{i}=\mathrm{i}+1 \\
& \text { n[i] <- dplyr::filter(frq1, GT == "1/1") } \%>\% \operatorname{nrow}()^{\star} 2 \\
& \mathrm{i}=\mathrm{i}+1 \\
& \text { n[i] <- dplyr::filter(frq2, GT == "0/1") \%> } \% \text { nrow }() \\
& \mathrm{i}=\mathrm{i}+1 \\
& \text { n[i] <- dplyr::filter(frq2, GT =="1/1") \%> } \% \text { nrow }()^{*} 2 \\
& \mathrm{i}=\mathrm{i}+1 \\
& \text { n[i] <- dplyr::filter(frq3, GT == "0/1") \%> } \operatorname{nrow}() \\
& \mathrm{i}=\mathrm{i}+1 \\
& \text { n[i] <- dplyr::filter(frq3, GT =="1/1") \%> } \% \text { nrow }()^{\star} 2 \\
& \mathrm{i}=\mathrm{i}+1 \\
& \text { n[i] <- dplyr::filter(frq4, GT =="0/1") \%> } \% \operatorname{nrow}() \\
& \mathrm{i}=\mathrm{i}+1 \\
& \text { soma[j, }]<-\operatorname{cbind}(\mathrm{n}[1],(\text { as.numeric }(\mathrm{n}[2])+ \\
& \text { as.numeric }(\mathrm{n}[3])),(\text { as.numeric }(\mathrm{n}[4])+\text { as.numeric }(\mathrm{n}[5])),(\text { as.numeric }(\mathrm{n}[6])+
\end{aligned}
$$$$
\text { as.numeric(n[7])), as.numeric(n[8])) }
$$

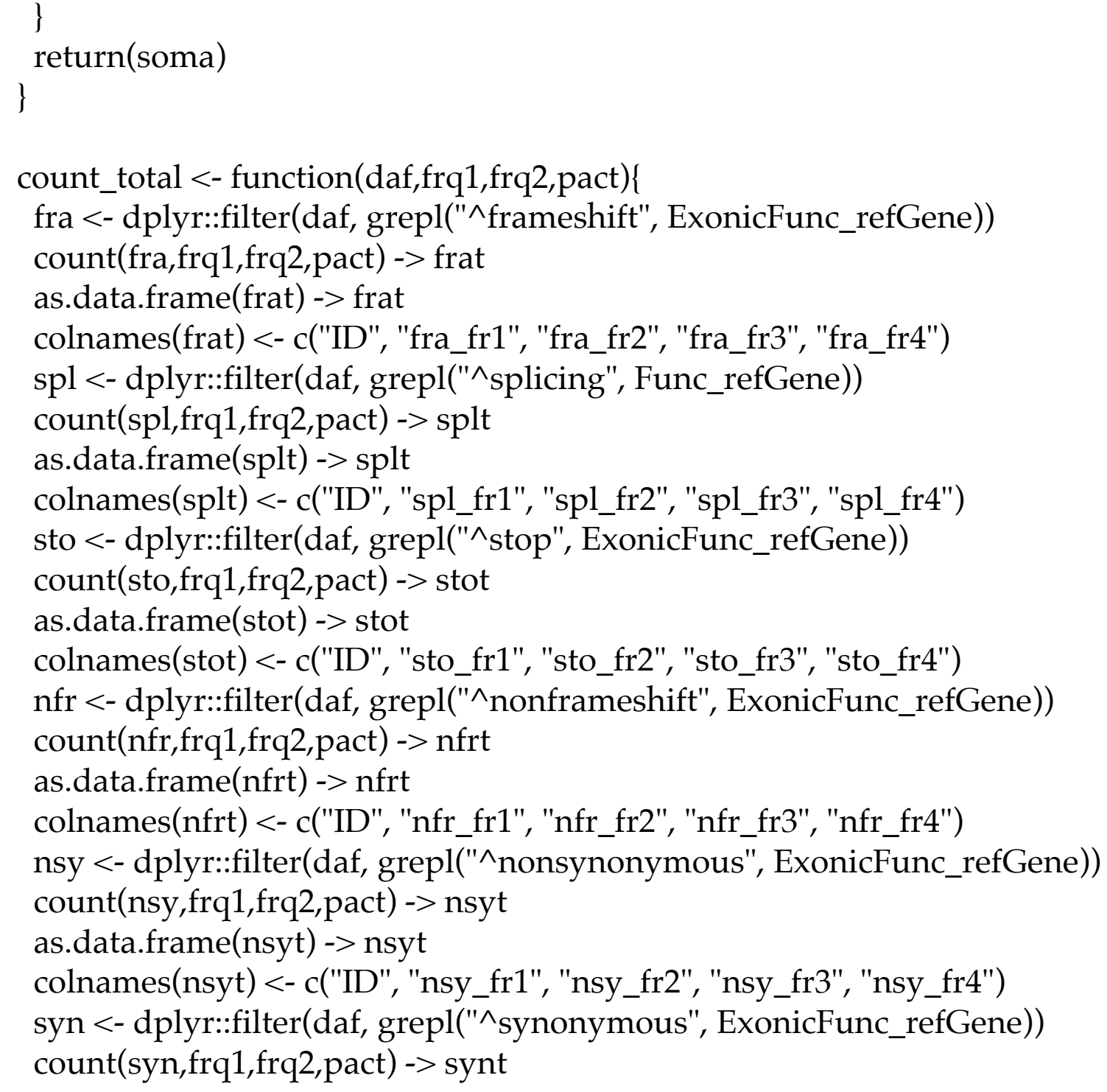




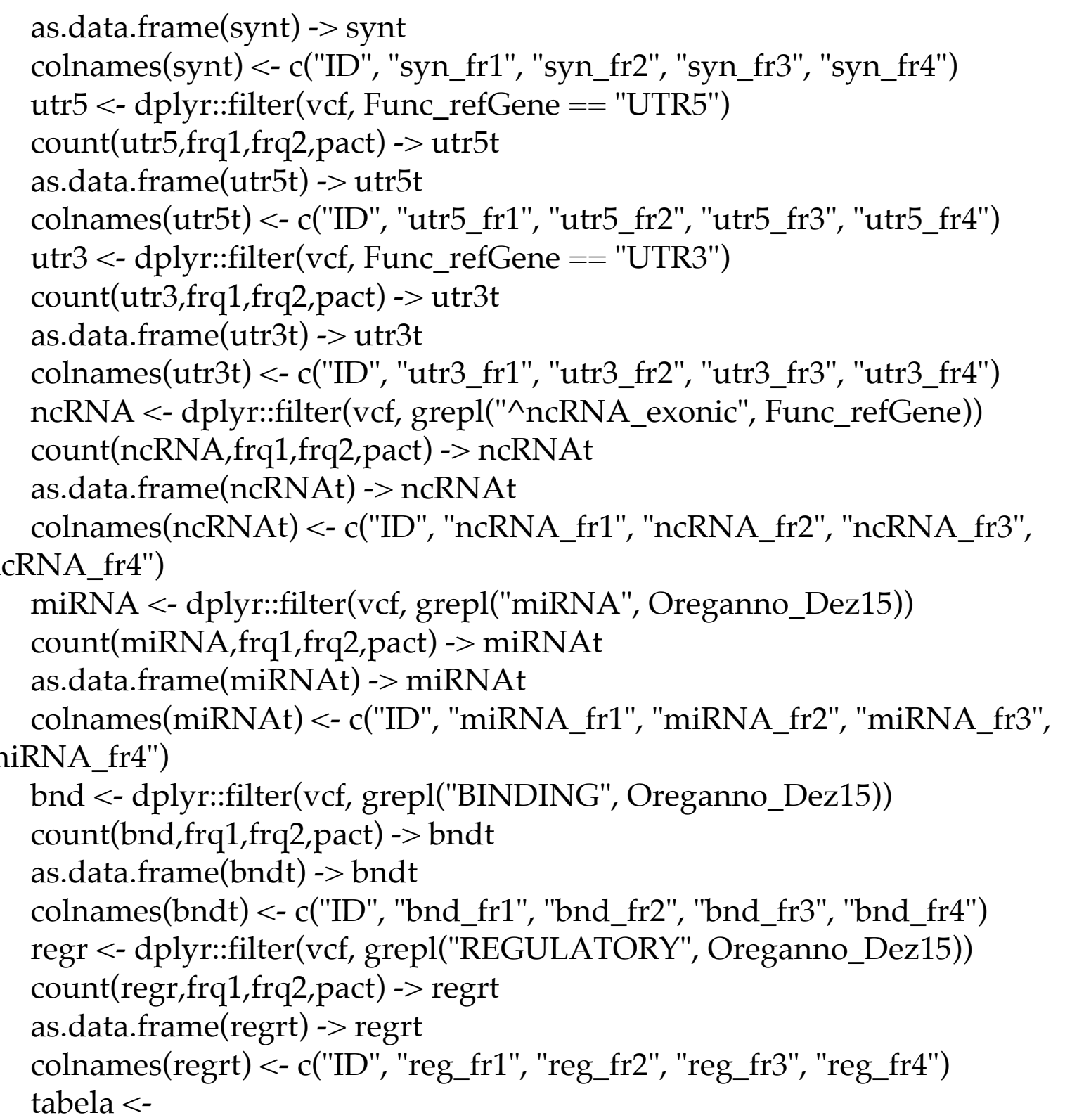

plyr::join_all(list(frat,splt,stot,nfrt,nsyt,synt,utr5t,utr3t,ncRNAt,miRNAt,bndt, regrt))

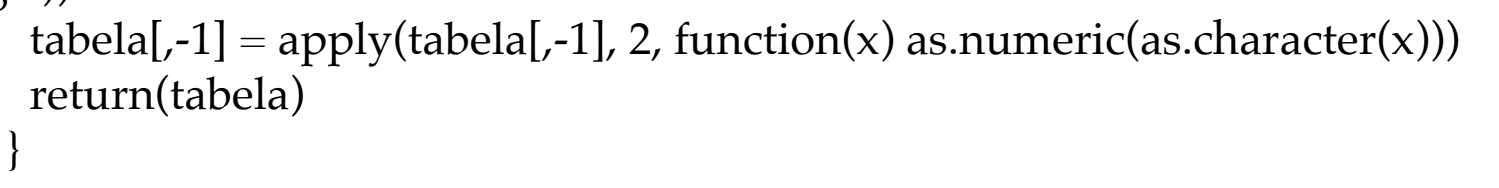

classvcf $<-$

c("character","integer","integer","character","character","factor","factor","factor" ,"factor","numeric","numeric","numeric","character","character","integer","char acter","character","character","character","character","character","character","ch aracter","character","character","character","character","character","character"," character","character","character","character","character","character","character ","character","character","character","character","character","character","charact er","character","character","character","character","character","character","chara cter","character","character","character","character","character","character","cha 
racter","character","character","character","character","character","character","c haracter","character","character","character","character","character","character", "character","character","character","character","character","character","characte r","character","character","character","character","character","character","charac ter","character","character","character","character","character","character","char acter","character","character","character","character","character","character","ch aracter","character","character","character","character","character","character"," character","character","character","character","character","character","character ","character","character","character","character","character","character","charact er","character","character","character","character","character","character","chara cter","character","character","character","character","character","character","cha racter","character","character","character","character","character","character","c haracter","character","character","character","character","character","character", "character","character","character","character","character","character","characte r","character","character","character","character","character","character","charac ter","character","character","character","character","character","character","char acter","character","character","character","character","character","character","ch aracter","character","character","character","character","character","character"," character","character","character","character","character","character","character ","character","character","character","character","character","character","charact er","character","character")

read.delim("bedcromatinaall.txt.anotado.cut.txt", sep = "\t", dec = ".", header $=\mathrm{T}$, stringsAsFactors $=\mathrm{F}$, check.names $=\mathrm{F}$, colClasses $=$ classvcf) $->$ vcf

read.table("dados_2016_06_15.txt") -> dados

dplyr::filter(dados, zen_code \%in\% names(vcf)) -> dadosval dplyr::filter(dadosval, diagnostico != "NA") -> dadosval

dadosval\$zen_code -> paciente

as.data.frame(paciente, col.names = "paciente") $->$ paciente

paciente $->$ pact

count_total(vcf, $0.05,0.01$,pact) -> cont merge(dadosval,cont, by.x = "zen_code", by.y = "ID") -> final final\$lof_fr3 <- final\$fra_fr3+final\$sto_fr3+final\$spl_fr3 final\$lof_fr4 <- final\$fra_fr4+final\$sto_fr4+final\$spl_fr4

write.table(final, "final.txt") 


\subsection{Attachment 24}

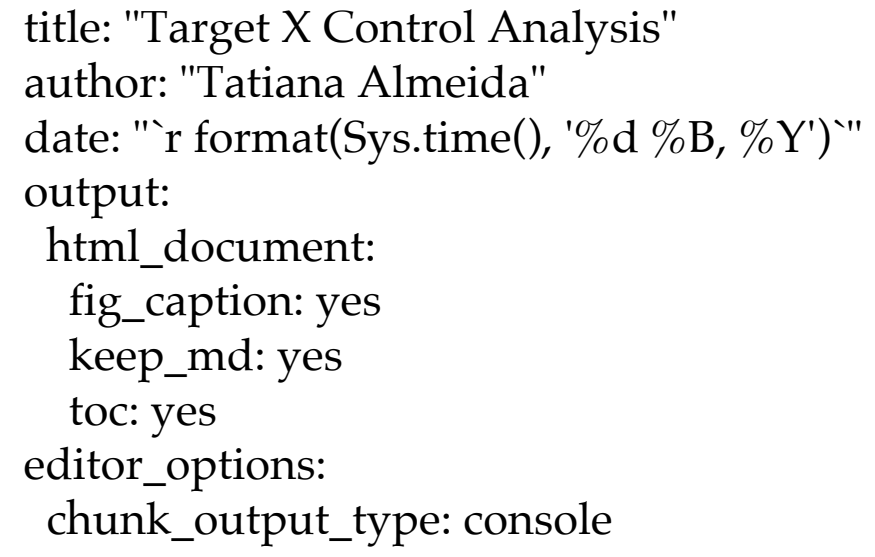

' $\{\mathrm{r}$ bibliotecas, echo $=$ FALSE, message $=$ FALSE, warning $=F A L S E ~\}$ \#\#\# library

library(dplyr)

library(magrittr)

library(ggplot2)

library(tidyr)

library(lazyeval)

library(openxlsx)

library(SNPRelate)

library(gridExtra)

library(devtools)

library(ggbiplot)

library(knitr)

library(caret)

library(rpart)

library(rpart.plot)

library(gmodels)

library(arules)

library(hmeasure)

library(pROC)

library(neuralnet)

library(GGally)

library(corrplot)

library(broom)

library(psych)

library(ggpubr)

library(e1071)

library(kableExtra) 
vcf.fn $<-"($ raw VCF file)"

“' $\{r$ função all_num, echo=FALSE,message=FALSE, warning=FALSE $\}$

all_num $<$-function(df,status,id) \{

nums <- sapply(df, is.numeric)

df_nu $<-$ df[,nums]

df_nu[,sapply(df_nu, function(v) var(v, na.rm=TRUE)!=0)] -> df_nu

cbind(df[,id],df_nu) -> df_num

colnames(df_num)[1]<- "paciente"

\#df_num[,sapply(df_num[-1], function(v) var(v, na.rm=TRUE)!=0)]

return(df_num)

\}

' $\{$ r função multiplot, echo=FALSE,message $=$ FALSE, warning=FALSE $\}$

multiplot $<-$ function (..., plotlist=NULL, file, cols $=1$, layout=NULL $)\{$

library(grid)

\# Make a list from the ... arguments and plotlist

plots $<-$ c(list $(. .$.$) , plotlist )$

numPlots $=$ length(plots)

\# If layout is NULL, then use 'cols' to determine layout

if (is.null(layout)) \{

\# Make the panel

\# ncol: Number of columns of plots

\# nrow: Number of rows needed, calculated from \# of cols

layout <- matrix $(\operatorname{seq}(1$, cols * ceiling(numPlots / cols $))$, ncol $=$ cols, nrow $=$ ceiling $($ numPlots $/$ cols $))$

\}

if (numPlots $==1)\{$

print(plots[[1]])

\} else \{

\# Set up the page

grid.newpage()

pushViewport(viewport(layout = grid.layout(nrow(layout),

ncol(layout))))

\# Make each plot, in the correct location

for (i in 1:numPlots) \{ 
\# Get the i,j matrix positions of the regions that contain this subplot matchidx $<-$ as.data.frame $($ which (layout $==\mathrm{i}$, arr.ind $=$ TRUE) $)$

$\operatorname{print}($ plots$[[i]], v p=\operatorname{viewport}($ layout.pos.row $=$ matchid $x \$$ row, layout.pos.col $=$ matchid $x \$$ col $)$ )

"' $\{r$ função aed, fig.height=10, fig.width=15,echo=FALSE,message=FALSE, warning=FALSE

aed $<$ - function $(\mathrm{df}, \mathrm{nc})\{$

all_num(df,alvo,"zen_code") -> df_num

df_num[,nc] $<-$ as.factor(df_num[,nc])

dplyr::rename_(df_num, target $=\mathrm{nc})->\mathrm{rdf}$

ggpairs(rdf[, which(names(rdf) \%in \%

c("target","fra_fr1","spl_fr1","sto_fr1","nfr_fr1","nsy_fr1","syn_fr1","utr5_fr1","u tr3_fr1","ncRNA_fr1","miRNA_fr1","bnd_fr1","reg_fr1"))],

mapping=ggplot2::aes(colour $=$ target, alpha $=.25)$ ) $->$ p1

ggpairs(rdf[, which(names(rdf) \% in\%

c("target","fra_fr2","spl_fr2","sto_fr2","nfr_fr2","nsy_fr2","syn_fr2","utr5_fr2","u tr3_fr2","ncRNA_fr2","miRNA_fr2","bnd_fr2","reg_fr2"))],

mapping=ggplot2::aes $($ colour $=$ target, alpha $=.25)) ~->$ p2

ggpairs(rdf[, which(names(rdf) \% in \%

c("target","fra_fr3","spl_fr3","sto_fr3","nfr_fr3","nsy_fr3","syn_fr3","utr5_fr3","u tr3_fr3","ncRNA_fr3","miRNA_fr3","bnd_fr3","reg_fr3"))],

mapping=ggplot2::aes $($ colour $=$ target, alpha $=.25))->$ p3

ggpairs(rdf[, which(names(rdf) \%in\%

c("target","fra_fr4","spl_fr4","sto_fr4","nfr_fr4","nsy_fr4","syn_fr4","utr5_fr4","u tr3_fr4","ncRNA_fr4","miRNA_fr4","bnd_fr4","reg_fr4"))],

mapping=ggplot2::aes $($ colour $=$ target, alpha=.25)) $->$ p4

ggpairs(rdf[, which(names(rdf) \% in\%

c("target","fra_fr1","fra_fr2","fra_fr3","fra_fr4","lof_fr3","lof_fr4"))],

mapping=ggplot2::aes(colour $=$ target, alpha $=.25))->$ p5

ggpairs(rdf[, which(names(rdf) \%in\%

c("target","sto_fr1","sto_fr2","sto_fr3","sto_fr4","lof_fr3","lof_fr4"))],

mapping=ggplot2::aes(colour $=$ target, alpha $=.25))->$ p6

ggpairs(rdf[, which(names(rdf) \% in\%

c("target","spl_fr1","spl_fr2","spl_fr3","spl_fr4","lof_fr3","lof_fr4"))],

mapping=ggplot2::aes(colour $=$ target, alpha=.25)) $->$ p7

ggpairs(rdf[, which(names(rdf) \% in \%

c("target","nfr_fr1","nfr_fr2","nfr_fr3","nfr_fr4"))],

mapping=ggplot2::aes (colour $=$ target, alpha $=.25))$-> p8 
ggpairs(rdf[, which(names(rdf) \%in\%

c("target","nsy_fr1","nsy_fr2","nsy_fr3","nsy_fr4"))],

mapping=ggplot2::aes $($ colour $=$ target, alpha $=.25))->$ p9

ggpairs(rdf[, which(names(rdf) \%in \%

c("target","syn_fr1","syn_fr2","syn_fr3","syn_fr4"))],

mapping=ggplot2::aes $($ colour $=$ target, alpha $=.25))->$ p10

ggpairs(rdf[, which(names(rdf) \%in\%

c("target","utr5_fr1","utr5_fr2","utr5_fr3","utr5_fr4"))],

mapping=ggplot2::aes $($ colour $=$ target, alpha $=.25))->$ p11

ggpairs(rdf[, which(names(rdf) \% in\%

c("target","utr3_fr1","utr3_fr2","utr3_fr3","utr3_fr4"))],

mapping=ggplot2::aes $($ colour $=$ target, alpha $=.25))->$ p12

ggpairs(rdf[, which(names(rdf) \%in\%

c("target","ncRNA_fr1","ncRNA_fr2","ncRNA_fr3","ncRNA_fr4"))],

mapping=ggplot2::aes $($ colour $=$ target, alpha $=.25))$-> p13

ggpairs(rdf[, which(names(rdf) \% in\%

c("target","miRNA_fr1","miRNA_fr2","miRNA_fr3","miRNA_fr4"))],

mapping=ggplot2::aes $($ colour $=$ target, alpha $=.25))->$ p14

ggpairs(rdf[, which(names(rdf) \%in\%

c("target","bnd_fr1","bnd_fr2","bnd_fr3","bnd_fr4"))],

mapping=ggplot2::aes $($ colour $=$ target, alpha $=.25))->$ p15

ggpairs(rdf[, which(names(rdf) \%in\%

c("target","reg_fr1","reg_fr2","reg_fr3","reg_fr4"))],

mapping=ggplot2::aes $($ colour $=$ target, alpha $=.25))->$ p16

return(list(p1,p2,p3,p4,p5,p6,p7,p8,p9,p10,p11,p12,p13,p14,p15,p16))

\}

plotpc $<-$ function (df) \{

all_num(df,alvo,"zen_code") -> df_num

select(df_num, -exoma, -paciente, -ID_pac, -nascimento) -> df_num2

corr.test(df_num2) $->\mathrm{c} 1$

plot $1<-$ function ()\{

corrplot(c1\$r, method = "circle", order = "original", type = "upper",

sig.level $=0.05$, tl.col $=$ "black", tl.srt $=45$, tl.cex $=0.7$ )

\}

plot $2<$ - function ()\{

corrplot (c1\$p, method = "circle", order = "original", type = "full", sig.level

$=0.05$, tl.col $=$ "black", tl.srt $=45$, tl.cex $=0.7, \mathrm{cl} \cdot \lim =\mathrm{c}(0,1))$

\}

return(list $($ plot $1=$ plot 1 , plot $2=$ plot 2$))$

\}

aed $2<$ - function(df) \{

all_num(df,alvo,"zen_code") -> df_num 


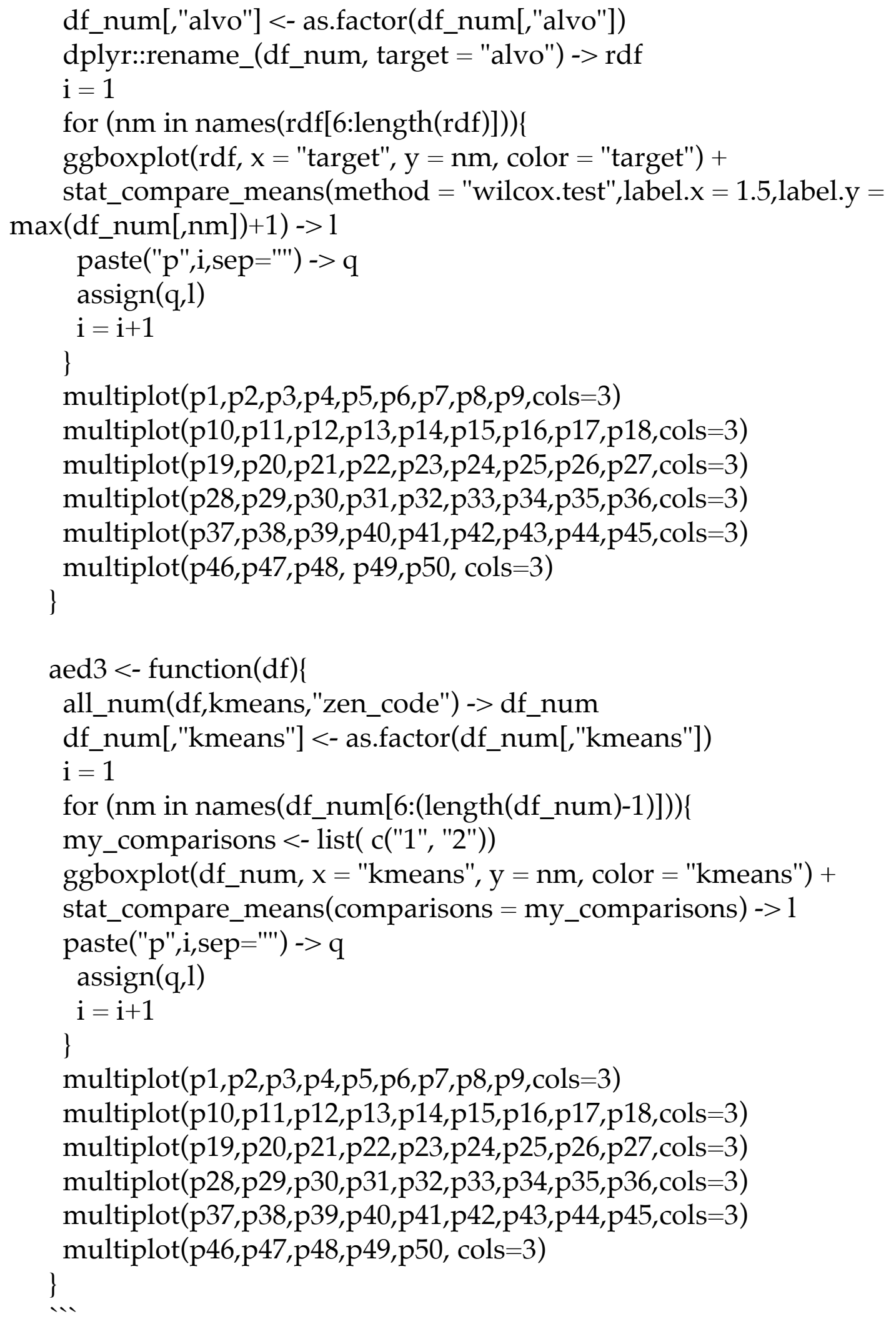

"' $\{r$ função hp_lof, echo=FALSE,message=FALSE, warning=FALSE $\}$ hp_lof $<-$ function $(\mathrm{df}, \mathrm{ct})\{$

ifelse(df\$alvo==1,"affected","control") -> df\$alvobi

if (var(df\$lof_fr3) != 0 \& var(df\$lof_fr4) !=0)\{ 
df\$lof_fr3d <- discretize(df\$lof_fr3, method = "frequency", categories = ct) table(df\$lof_fr3d,df $\$$ alvobi) $->\mathrm{m} 3$

prop.table $(\mathrm{m} 3,2)->\mathrm{m} 3 \mathrm{p}$

round $(m 3 p$, digits $=3) \rightarrow m 3 p$

df $\$$ lof_fr4d <- discretize $($ df $\$$ lof_fr4, method = "frequency", categories = ct) table(df $\$$ lof_fr4d,df $\$$ alvobi) $->\mathrm{m} 4$

prop.table $(\mathrm{m} 4,2) \rightarrow \mathrm{m} 4 \mathrm{p}$

round $(\mathrm{m} 4 \mathrm{p}$, digits $=3) \rightarrow \mathrm{m} 4 \mathrm{p}$

ifelse $(\operatorname{dim}(\mathrm{m} 3)[1]+\operatorname{dim}(\mathrm{m} 3)[2]>3$, chisq.test $(\mathrm{m} 3)[[3]]$,"sem cáclulo de Chisquare") -> f1

round(as.numeric(f1), digits $=3)->\mathrm{f} 1$

ifelse $(\operatorname{dim}(\mathrm{m} 4)[1]+\operatorname{dim}(\mathrm{m} 4)[2]>3$, chisq.test $(\mathrm{m} 4)[[3]]$,"sem cáclulo de Chisquare") -> f2

round (as.numeric $(\mathrm{f} 2)$, digits $=3)->$ f2

df\$lof_fr3d <- discretize (df\$lof_fr3, method = "fixed", categories = $\left.c\left(0,1, \max \left(d f \$ l o f \_f r 3\right)\right)\right)$

table(df\$lof_fr3d,df\$alvobi) -> m5

prop.table $(\mathrm{m} 5,2)->\mathrm{m} 5 \mathrm{p}$

round $(m 5 p$, digits $=3) \rightarrow m 5 p$

df\$lof_fr4d <- discretize (df\$lof_fr4, method = "fixed", categories = $\mathrm{c}(0,1, \max (\mathrm{df} \$$ lof_fr3) $))$

table(df\$lof_fr4d,df\$alvobi) -> m6

prop.table $(\mathrm{m} 6,2)->\mathrm{m} 6 \mathrm{p}$

round $(\mathrm{m} 6 \mathrm{p}$, digits $=3) \rightarrow \mathrm{m} 6 \mathrm{p}$

ifelse $(\operatorname{dim}(\mathrm{m} 5)[1]+\operatorname{dim}(\mathrm{m} 5)[2]>3$, chisq.test $(\mathrm{m} 5)[[3]]$,"sem cáclulo de Chisquare") -> f3

round(as.numeric(f3), digits $=3) \rightarrow \mathrm{f} 3$

ifelse $(\operatorname{dim}(\mathrm{m} 6)[1]+\operatorname{dim}(\mathrm{m} 6)[2]>3$, chisq.test $(\mathrm{m} 6)[[3]]$,"sem cáclulo de Chisquare") -> f4

round(as.numeric(f4), digits $=3)->f 4$

df\$lof_fr3d <- discretize (df\$lof_fr3, method = "cluster", categories = ct)

table(df $\$$ lof_fr3d,df\$alvobi) $\rightarrow>\mathrm{m} 7$

prop.table $(\mathrm{m} 7,2)->\mathrm{m} 7 \mathrm{p}$

round $(m 7 p$, digits $=3) \rightarrow m 7 p$

df $\$$ lof_fr4d <- discretize $($ df\$lof_fr4, method = "cluster", categories = ct)

table(df\$lof_fr4d,df $\$$ alvobi) $->\mathrm{m} 8$

prop.table $(\mathrm{m} 8,2)->\mathrm{m} 8 \mathrm{p}$

round $(m 8 p$, digits $=3) \rightarrow m 8 p$

ifelse $(\operatorname{dim}(\mathrm{m} 7)[1]+\operatorname{dim}(\mathrm{m} 7)[2]>3$, chisq.test $(\mathrm{m} 7)[[3]]$,"sem cáclulo de Chisquare") -> f5

round(as.numeric(f5), digits $=3)->\mathrm{f} 5$

ifelse( $\operatorname{dim}(\mathrm{m} 8)[1]+\operatorname{dim}(\mathrm{m} 8)[2]>3$, chisq.test(m8)[[3]],"sem cáclulo de Chi- 
square") -> f6

round(as.numeric(f6), digits $=3$ ) $->\mathrm{f} 6$

tabela <- rbind(m3,m3p,m4,m4p,m5,m5p,m6,m6p,m7,m7p,m8,m8p)

tabela $<-$ cbind (tabela, ChiSquare $=$

c(f1,f1,f1,f1,f2,f2, f2,f2,f3,f3,f3,f3,f4,f4,f4,f4,f5,f5,f5,f5,f6, f6, f6, f6 ))

as.data.frame(tabela) $->$ tabela

names $<$ - rownames(tabela)

rownames(tabela) $<-$ NULL

tabela $<$ - cbind(names, tabela)

ifelse(tabela\$ChiSquare == "NaN",99, tabela\$ChiSquare) $->$

tabela\$ChiSquare

colnames(tabela) <- c("Number of LoF","Affected","Control","ChiSquare")

options (knitr.table.format $=$ "html")

tabela $\%>\%$

mutate(

ChiSquare $=$ cell_spec $($ ChiSquare, color $=$ ifelse $($ ChiSquare $<.05$, "red", "black"))) \%>\%

select('Number of LoF',Affected,Control,ChiSquare) \%>\%

kable $($ escape $=\mathrm{F}) \%>\%$

kable_styling(bootstrap_options = c("striped", "hover", "condensed",

"responsive", position = "left")) $\%>\%$

collapse_rows (columns $=4) \%>\%$

group_rows("Grouping Method: Frequency",1,8) \%>\%

group_rows("Rare variants",1,4) \%>\%

group_rows("Singletons",5,8) \%>\%

group_rows("Grouping Method: Presence versus Absence",9,16) \%>\%

group_rows("Rare variants",9,12) \%>\%

group_rows("Singletons",13,16) \%>\%

group_rows("Grouping Method: Kmeans",17,24) \%>\%

group_rows("Rare variants",17,20) \%>\%

group_rows("Singletons",21,24)

\}

\}

lof_roc $<$ - function $(\mathrm{df})\{$

cbind(df\$zen_code,df\$alvo,df\$lof_fr3,df\$lof_fr4) $\%>\%$ as.data.frame() -> pac_lof

colnames(pac_lof) <- c("zen_code", "alvo", "lof_fr3","lof_fr4")

$\min ($ df $\$$ lof_fr3) $->\mathrm{mn}$

$\max ($ df $\$$ lof_fr3) $->\mathrm{mx}$

$\operatorname{matrix}($ data $=\mathrm{NA}$, nrow $=(\mathrm{mx}-\mathrm{mn})+1, \mathrm{ncol}=2)->$ roc $\_1$

colnames(roc_1) <- c("TVP","TFP") 
rownames(roc_1) $<-\mathrm{mn}: \mathrm{mx}$

$\mathrm{j}<-1$

for (i in $m n: m x)\{$

filter(pac_lof, alvo == 1 \& lof_fr3 $>=$ i) $\%>\%$ nrow ()$/$ nrow $($ filter(pac_lof, alvo $==1))->$ roc_1 $[\mathrm{j}, 1]$

filter(pac_lof, alvo $==0 \&$ lof_fr3 $>=\mathrm{i}) \%>\% \operatorname{nrow}() / \operatorname{nrow}\left(\right.$ filter$\left(p a c \_l o f\right.$, alvo $==0))->$ roc_1 $1 \mathrm{j}, 2]$

$\mathrm{j}<-\mathrm{j}+1$

$\min (\mathrm{df} \$$ lof_fr 4$)->\mathrm{mn}$

$\max ($ df $\$$ lof_fr4) $->\mathrm{mx}$

$\operatorname{matrix}($ data $=\mathrm{NA}, \mathrm{nrow}=(\mathrm{mx}-\mathrm{mn})+1, \mathrm{ncol}=2)->$ roc_2

colnames(roc_2) <- c("TVP","TFP")

rownames(roc_2) $<-\mathrm{mn}: \mathrm{mx}$

$\mathrm{j}<-1$

for (i in $m n: m x)\{$

filter(pac_lof, alvo $==1 \&$ lof_fr $4>=\mathrm{i}) \%>\% \operatorname{nrow}() / \operatorname{nrow}($ filter(pac_lof, alvo $==1))->$ roc_2[j,1]

filter(pac_lof, alvo $==0 \&$ lof_fr $4>=\mathrm{i}) \%>\% \operatorname{nrow}() / \operatorname{nrow}($ filter(pac_lof, alvo $==0))->$ roc_2 $[\mathrm{j}, 2]$

$\mathrm{j}<-\mathrm{j}+1$

plot(roc_1[,2],roc_1[,1], ylab = "True Positive Rate", xlab = "False Positive Rate",main = "Rare variants", abline $(\mathrm{a}=0, \mathrm{~b}=1))->$ pl1

plot(roc_2[,2],roc_2[,1], ylab = "True Positive Rate", xlab = "False Positive Rate", main = "Singleton variants", abline $(\mathrm{a}=0, \mathrm{~b}=1))->$ pl2

\# return(list(pl1,pl2))

\}...

' $\{r$ função spring2, echo=FALSE,message=FALSE, warning=FALSE $\}$ spring2 <- function(df,dados) \{

colnames(df)<- c("paciente","gene","line","rank","pvalue","qvalue")

merge(dados,df, by.x = "zen_code", by.y = "paciente") -> spr

spr\$logqv <- round(-log(as.numeric(spr\$qvalue)), digits $=0$ )

ggplot(spr) +

geom_violin(aes(x=as.factor(alvo), $\mathrm{y}=\operatorname{logqv}))+$

labs(x="'", y="log(q-value)", title="Q-value distribution") -> g1

table(spr\$zen_code, spr\$logqv) $\%>\%$ as.data.frame()->a

colnames(a)<- c("zen_code","logqv","cont")

merge(dados, a, by $=$ "zen_code") $->$ b

filter $(b$, alvo $==0)->$ ntea

filter $(\mathrm{b}$, alvo $==1)->$ tea

aggregate $($ data $=$ ntea, cont $\sim \operatorname{logqv}$, mean $)->c$ 


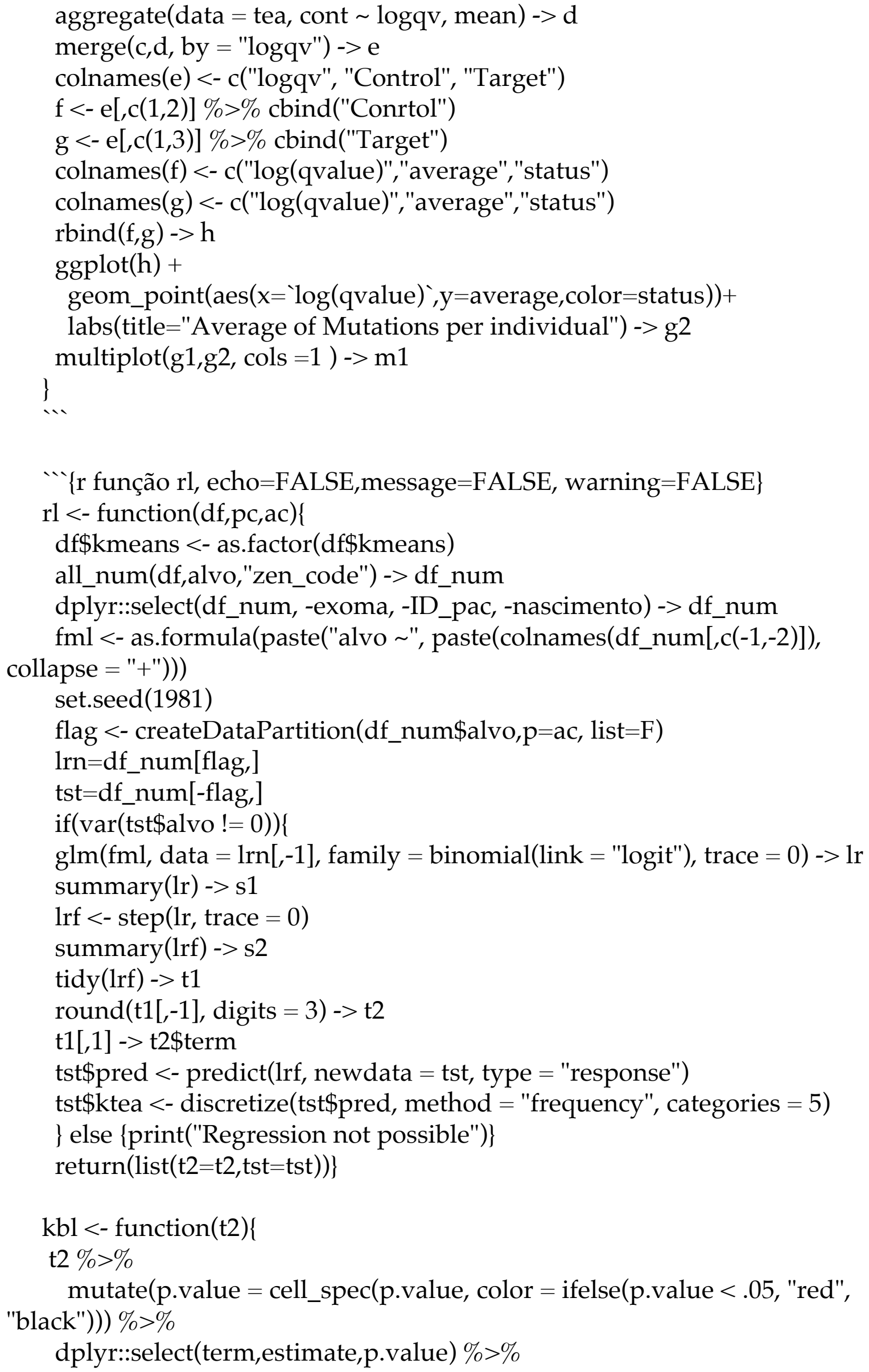


kable $($ escape $=\mathrm{F}) \%>\%$

kable_styling(bootstrap_options = c("striped", "hover", "condensed", font_size =6))\}

"' $\{r$ função tree, echo=FALSE, message=FALSE, warning=FALSE $\}$

tree $<-$ function $(\mathrm{df}, \mathrm{pc})\{$

df\$kmeans $<-$ as.factor(df\$kmeans)

all_num(df,alvo,"zen_code") -> df_num

dplyr::select(df_num, -exoma, -ID_pac, -nascimento) -> df_num

ifelse(df_num\$alvo ==1,"Target","Control") -> df_num\$tea_bi

$\mathrm{fml}<-$ as.formula(paste("tea_bi ", paste(colnames(df_num[,-

$\mathrm{c}(1,2$,length(df_num) $)])$, collapse $="+")))$

set.seed(1981)

flag $<-$ createDataPartition $\left(\mathrm{df} \_\right.$num\$alvo, $\mathrm{p}=\mathrm{pc}$, list $=\mathrm{F}$ )

lrn=df_num[flag,]

if (var(lrn\$alvo) !=0)\{

ad $1<-\operatorname{rpart}($ data $=1 \mathrm{rn}, \mathrm{fml})$

$\operatorname{prp}(\operatorname{ad} 1$, type $=2$, extra $=100, \mathrm{nn}=\mathrm{T}$, fallen.leaves $=\mathrm{T}$, branch.col $=$ "red",

branch.lty $=5$, box.col = c("white","green")) -> a1

printcp $(\operatorname{ad} 1)->$ e1

\} else \{

a1 $<-$ NULL

e1 $<-$ NULL

ad $1<-$ NULL

\}

return(list(a1,e1,ad1))

\}

prune $<-$ function $($ ad1, cut $)\{$

ad $2<$-prune $($ ad $1, \mathrm{cp}=$ cut $)$

$\operatorname{prp}(\operatorname{ad} 2$, type $=2$, extra $=104, \mathrm{nn}=\mathrm{T}$, fallen.leaves $=\mathrm{T}$, branch.col

$="$ red",branch.lty $=5$, box.col $=c($ "white",'green')) $->\mathrm{a} 2$

return $(\operatorname{list}(\mathrm{ad} 2, \mathrm{a} 2))$

\}

treetest $<$ - function $(\mathrm{df}, \mathrm{ad}, \mathrm{pc})\{$

df $\$$ kmeans $<-$ as.factor(df $\$$ kmeans)

all_num(df,alvo,"zen_code") -> df_num

dplyr::select(df_num, -exoma, -ID_pac, -paciente, -nascimento) ->df_num

ifelse(df_num\$alvo ==1,"Target","Control") -> df_num\$tea_bi

set.seed(1981)

flag $<-$ createDataPartition $\left(\mathrm{df} \_\right.$num $\$$ alvo, $\mathrm{p}=\mathrm{pc}$, list $=\mathrm{F}$ )

tst=df_num[-flag,] 


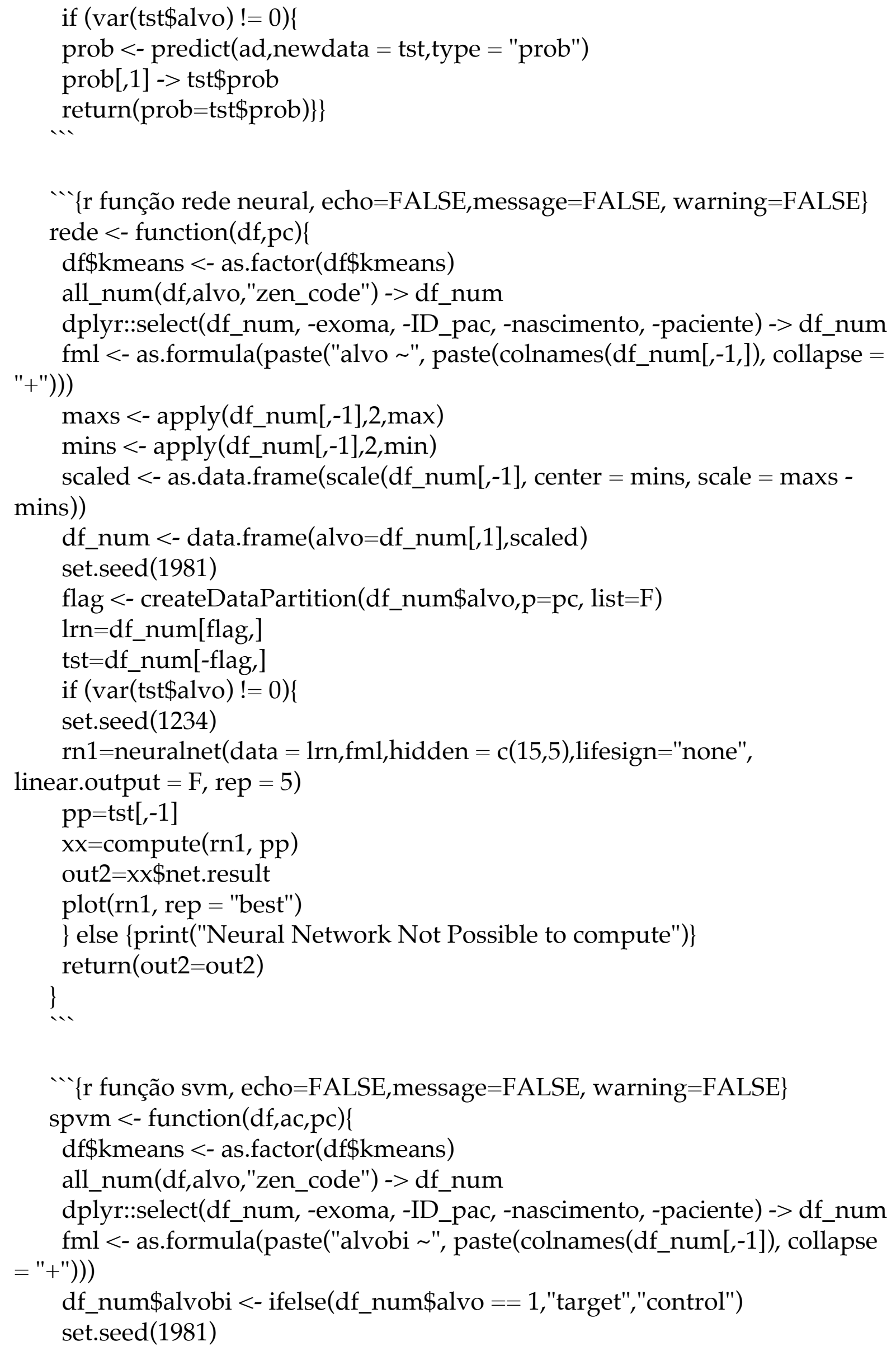

' " $\{r$ função rede neural, echo=FALSE, message=FALSE, warning=FALSE $\}$ 
flag $<-$ createDataPartition $\left(\mathrm{df}_{-}\right.$num $\$$ alvo, $\mathrm{p}=\mathrm{ac}$, list=F)

lrn=df_num[flag, ]

tst=df_num[-flag,

if ( $\operatorname{var}($ tst\$alvo) $!=0)\{$

sv $<-\operatorname{svm}($ fml,data $=$ lrn, kernel = "linear", type = "C-classification", probability $=\mathrm{T}$ )

$\mathrm{pp}=\mathrm{tst}[,-1]$

pred $<-$ predict $(\mathrm{sv}, \mathrm{pp}$, probability $=\mathrm{T})$

attr(pred, 'probabilities')[,2] -> tst\$pred

p1 <- ggplot(tst) +

geom_point(aes( $\mathrm{x}=\mathrm{as}$. factor(alvo), $\mathrm{y}=$ pred, $\operatorname{color}=\mathrm{as}$. factor(alvo $)$ ), position $=$ "jitter") +

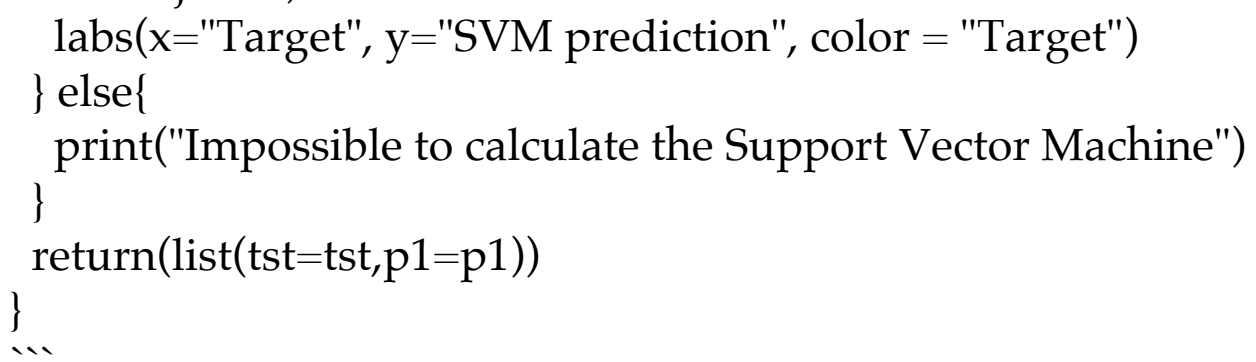

"' $\{r$ função hms, echo=FALSE, message=FALSE, warning=FALSE $\}$ hms $<$ - function(tst,hdf)\{

HMeasure(tst, hdf, severity.ratio $=0.75) \$$ metrics $->$ met round $($ met, digits $=3)->$ met

met $\%>\%$ mutate

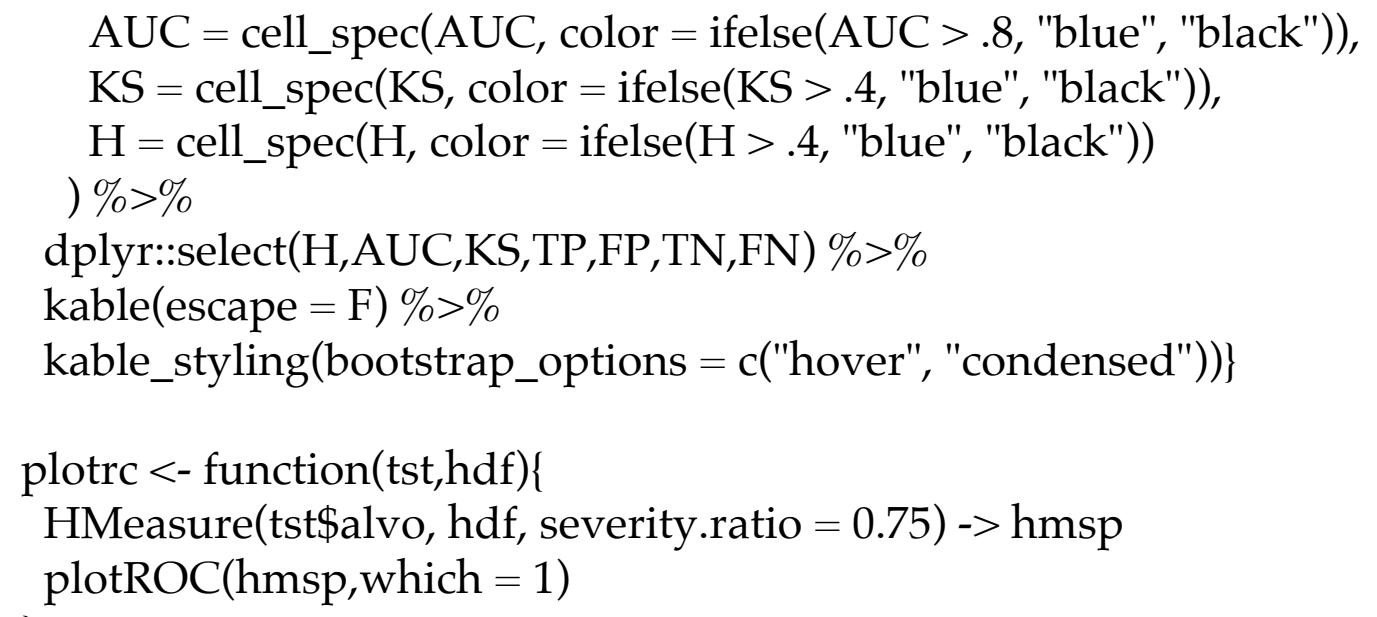

"' $\{r$ função kmeans, echo=FALSE, message=FALSE, warning=FALSE $\}$ $\mathrm{km}<-$ function $(\mathrm{df})\{$ all_num(df,alvo,"zen_code") -> df_num 
dplyr::select(df_num, -exoma, -ID_pac, -nascimento) -> df_num set.seed(1981)

k.max $<-15$

wss $<-\operatorname{sapply}(1: k \cdot m a x$, function $(k)\{k$ means (df_num[,-1], k, nstart=50,iter.max $=15$

)\$tot.withinss\})

plot(1:k.max, wss,

type $=" b "$, pch $=19$, frame $=$ FALSE,

xlab="Number of clusters K",

ylab="Total within-clusters sum of squares")

\}

$\mathrm{kb}<-$ function $(\mathrm{df}, \mathrm{kn})\{$

all_num(df,alvo,"zen_code") -> df_num

dplyr::select(df_num, -exoma, -ID_pac, -nascimento) -> df_num set.seed(1981)

wss $<-$ kmeans(df_num[,-1], kn, nstart=50,iter.max = 15 )\$cluster return(wss)

\}

' '\{r função pcaf, echo=FALSE, message=FALSE, warning=FALSE $\}$

pcaf $<$ - function(df,nome) \{

df\$kmeans $<-$ as.factor (df $\$$ kmeans)

all_num(df,alvo,"zen_code") -> df_num

select(df_num, -exoma, -ID_pac, -nascimento, -paciente) -> df_num

prcomp (df_num[,-1], center $=$ TRUE, scale $=$ TRUE) $->$ pca_df

$\mathrm{k}<-$ ggbiplot(pca_df, obs.scale $=1$, var.scale $=1$, groups $=$

as.factor(df\$alvo), ellipse $=$ TRUE, circle $=$ TRUE)

$\mathrm{k}<-\mathrm{k}+$ scale_color_discrete(name $="$ ")

$\mathrm{k}<-\mathrm{k}+$ theme(legend.direction $=$ 'horizontal', legend.position $=$ 'top')

$1<-$ ggbiplot(pca_df, obs.scale $=1$, var.scale $=1$, groups $=$

as.factor (df\$alvo), ellipse $=$ TRUE, circle $=$ TRUE, choices $=2: 3$ )

$1<-1+$ scale_color_discrete(name $="$ )

$1<-1+$ theme (legend.direction $=$ 'horizontal', legend.position = 'top')

grid.arrange $(\mathrm{k}, \mathrm{l}, \mathrm{ncol}=2)\}$

“' $\{r$ função pca_vcf, echo=FALSE,message=FALSE, warning=FALSE $\}$

pca_vcf $<$ - function(genofile, out, dados) \{

tea_pca<-snpgdsPCA(genofile)

as.data.frame(cbind(tea_pca\$sample.id,tea_pca\$eigenvect[,1],tea_pca\$eige nvect[,2],tea_pca\$eigenvect[,3])) -> a

left_join(a,dados, by =c("V1" = "zen_code")) -> data_pca 
dplyr::filter(data_pca, !V1\%in\% out) -> data2_pca

$\mathrm{k}<-$ ggplot $($ data $=$ data2_pca,

$\operatorname{aes}(\mathrm{x}=$ as.numeric $(\operatorname{as} . \operatorname{character}(\mathrm{V} 2)), \mathrm{y}=\operatorname{as}$.numeric $(\operatorname{as}$.character $(\mathrm{V} 3))))+$ geom_point ()$+$

geom_text(aes $($ label $=$ data2_pca\$V1) $)+$

geom_point $($ data $=$ data2_pca[data2_pca $\$ a l v o==0],, c o l=3)+\#$ verde

geom_point $($ data $=$ data2_pca[data2_pca\$alvo $==1],, c o l=2)+\#$

vermelho

labs (x="PC1", y="PC2")

$1<-$ ggplot(data $=$ data2_pca,

aes $(\mathrm{x}=$ as.numeric $($ as.character $(\mathrm{V} 3)), \mathrm{y}=\operatorname{as}$.numeric $($ as.character $(\mathrm{V} 4))))+$

geom_point ()$+$

geom_text $($ aes $($ label $=$ data2_pca\$V1) $)+$

geom_point $($ data $=$ data2_pca[data2_pca $\$$ alvo $==0],, c o l=3)+$ \# verde

geom_point $($ data $=$ data2_pca[data2_pca\$alvo $==1],, c o l=2)+\#$

vermelho

$\operatorname{labs}(\mathrm{x}=$ ="PC2", $\mathrm{y}=$ ="PC3")

grid.arrange $(\mathrm{k}, \mathrm{l}, \mathrm{ncol}=2)$

\}

' $\{r$ função $k$ comp, echo=FALSE,message $=$ FALSE, warning=FALSE $\}$

kmcomp $<-$ function $(\mathrm{df}$, nome,up,pc,ct $)\{$

data1 $<-$ NULL

data $2<-$ NULL

data $3<-$ NULL

data $4<-$ NULL

data $5<-$ NULL

data6 $<-$ NULL

set.seed(1981)

for (i in 1:up)

filter(df, kmeans $==\mathrm{i})->$ df1

if ( $\operatorname{var}($ df1\$alvo $) !=0)\{$

if (var(df1\$lof_fr3) != 0 \& var(df1\$lof_fr4) $!=0)\{$

hp_lof(df1,ct) $\rightarrow$ lofr

lof_roc(df1) -> lofroc

\} else \{print("No loss-of-fcuntion mutation") -> lofr; lofroc $<-$ NULL\}

$\mathrm{rl}(\mathrm{df} 1, .5, \mathrm{pc})->\mathrm{rlr}$ 


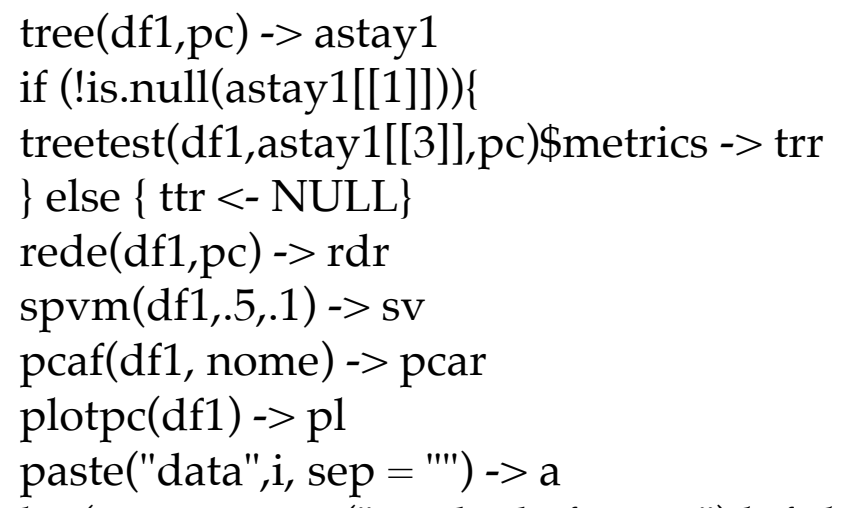

list(nome,i,print("Perda de função"),lofr,lofroc, print("regressão logística"), rlr, print("Árvore de decisão"), trr,print("rede neural"), rdr,print("Support Vector Machine"),sv,print("Componente principal"), pcar, pl\$plot1()) -> b

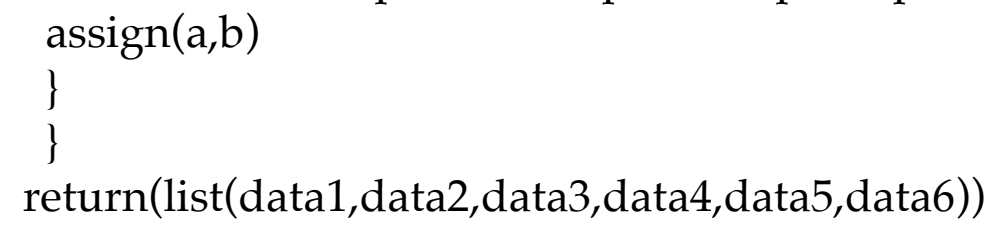

$\mathrm{nfr}<-$ aggregate$\left(\mathrm{nfr} \_f r 1+\mathrm{nfr} \_f r 2+n f r \_f r 3+n f r \_f r 4 \sim\right.$ zen_code, data $=$ final,

utr5 <- aggregate(utr5_fr1+utr5_fr2+utr5_fr3+utr5_fr4 $\sim$ zen_code, data $=$ final, sum) 
utr3 <- aggregate(utr3_fr1+utr3_fr2+utr3_fr3+utr3_fr4 zen_code, data = final, sum)

ncRNA <- aggregate(ncRNA_fr1+ncRNA_fr2+ncRNA_fr3+ncRNA_fr4 zen_code, data = final, sum)

miRNA <- aggregate(miRNA_fr1+miRNA_fr2+miRNA_fr3+miRNA_fr4 zen_code, data $=$ final, sum)

bnd $<-$ aggregate(bnd_fr1+bnd_fr2+bnd_fr $3+$ bnd_fr $4 \sim$ zen_code, data $=$ final, sum)

reg <- aggregate(reg_fr1+reg_fr2+reg_fr3+reg_fr4 $\sim$ zen_code, data $=$ final, sum)

$\operatorname{expl}<-$

join_all(list(syn,fra,sto,spl,nsy,nfr,utr5,utr3,ncRNA,miRNA,bnd,reg))

colnames $(\operatorname{expl})<-$

c("zen_code","Synonymous","Frameshift","Stopgainloss","Splicing","Nonsynon ymous",,"Nonframeshift","UTR5","UTR3","ncRNA","miRNA","Binding","Regul atory")

p1 <- ggplot $($ data $=\operatorname{expl}$, aes $(x=$ Frameshift $))+$

geom_histogram()

p2 <- ggplot $($ data $=\operatorname{expl}$, aes $(\mathrm{x}=$ Stopgainloss $))+$

geom_histogram()

p3 $<-$ ggplot $($ data $=$ expl, aes $(x=$ Splicing $))+$

geom_histogram()

p4 <- ggplot $($ data $=\operatorname{expl}$, aes $(x=$ Nonframeshift $))+$

geom_histogram()

p5 <- ggplot $($ data $=\operatorname{expl}$, aes $(x=$ Nonsynonymous $))+$

geom_histogram()

p6 <- ggplot(data $=\operatorname{expl}$, aes $(\mathrm{x}=$ Synonymous $))+$

geom_histogram()

p7 <- ggplot $($ data $=$ expl, aes $(x=$ UTR5 $))+$

geom_histogram()

p8 <- ggplot $($ data $=\operatorname{expl}$, aes $(x=$ UTR3 $))+$

geom_histogram()

p9 <- ggplot $($ data $=\operatorname{expl}$, aes $(x=$ ncRNA $))+$

geom_histogram()

p10 <- ggplot $($ data $=$ expl, aes $(x=$ miRNA $))+$

geom_histogram()

p11 <- ggplot $($ data $=\operatorname{expl}$, aes $(x=$ Binding $))+$

geom_histogram()

p12<- ggplot $($ data $=\operatorname{expl}$, aes $(x=$ Regulatory $))+$

geom_histogram()

multiplot(p1,p2,p3,p4,p5,p6, cols =3)

multiplot(p7,p8,p9,p10,p11,p12,cols=3) 
\# \# Kmeans

' $\{\mathrm{r}$ kmeans, echo=FALSE,message $=$ FALSE, warning $=$ FALSE $\}$ $\mathrm{km}$ (final)

$\mathrm{kb}($ final,2) -> final\$kmeans

\# read.table("../ all/kmeans.txt") -> a

\# colnames(a) <- "kmeans"

\# cbind(final,a) -> final

\# \# Correlation matrices

\#\#\# Correlation matrix for all individuals

' $\{$ r cma, echo=FALSE, message $=$ FALSE, warning $=$ FALSE $\}$

plotpc(final) $->$ pl

pl\$plot1()

\#\#\# P-value matrix for all individuals

' $\{\mathrm{r}$ pma, echo=FALSE, message $=$ FALSE, warning $=$ FALSE $\}$

pl\$plot2()

\#\# \# Correlation matrix for target individuals

' ' $\{\mathrm{r} \mathrm{cmt}$, echo=FALSE,message $=$ FALSE, warning $=$ FALSE $\}$

plotpc(final[final\$alvo $==1]),->$ pla

pla\$plot1()

\#\#\# Correlation matrix for control individuals

' $\{$ r cmc, echo=FALSE, message $=$ FALSE, warning $=$ FALSE $\}$

plotpc(final[final $\$$ alvo $==0]),->$ plc

plc\$plot1()

\# \# Comparison for each variant between affected and control

' $\{\mathrm{r}$ ea, echo $=$ FALSE, message $=$ FALSE, warning $=$ FALSE, fig.height $=10$, fig.width $=15$, \}

aed2(final) 
\#\# Comparison for each variant for each kmeans cluster

' $\{$ r eac, echo=FALSE, message=FALSE, warning=FALSE, fig.height=10, fig.width $=15$,

aed3(final)

…

\# Comparison analysis

\#\# Loss-of-function grouping

"' $\{r$ hp_lof, echo=FALSE, message=FALSE, warning=FALSE $\}$

hp_lof(final,2)

\#\# Loss-of-function ROC curve

' $\{r$ lof_roc, echo=FALSE,message=FALSE, warning=FALSE $\}$

lof_roc(final)

\#\# Comparison of Missense Mutations

\#\#\# Rare mutations

"' $\{$ r spring rara, echo=FALSE, message $=$ FALSE, warning=FALSE $\}$ read.table("rank1_rara.txt", sep = "\t", dec = ".",fill = T) -> springr spring2(springr,dados)

\#\#\# Singleton mutations

"' $\{r$ spring privativa, echo=FALSE, message=FALSE, warning=FALSE $\}$ read.table("rank1_privativa.txt", sep = "\t", dec = ".",fill = T) -> springp spring2(springp,dados)

\#\# Logistic Regression

' " $\{$ r regressão, echo $=$ FALSE, message $=$ FALSE, warning $=$ FALSE $\}$

rl(final, .5,.5) $->$ rl1

$\mathrm{kbl}(\mathrm{r} 11 \$ \mathrm{t} 2)$ 
\#\# Decicion Tree

' $\{r$ arvore, echo=FALSE, message $=$ FALSE, warning $=$ FALSE $\}$ tree(final,.5) -> afinal

treetest(final, afinal[[3]],.5) -> tr

$\cdots$

\#\# Neural Network

"' $\{\mathrm{r}$ rede neural, echo=FALSE, message=FALSE, warning=FALSE $\}$

rede(final,.5) $->\mathrm{nn}$

\#\# Support Vector Machine

' $\{$ r svm, echo=FALSE, message $=$ FALSE, warning=FALSE $\}$

spvm(final,.5,.1) -> sp1

$\mathrm{sp} 1 \$ \mathrm{p} 1$

\#\# Classification Performance

"' $\{\mathrm{r}$ clp, echo=FALSE, message=FALSE, warning=FALSE $\}$

hdf $<-$ data.frame(LR=rl1\$tst['pred'],DT=tr,NN=nn,SVM=sp1\$tst['pred'])

colnames(hdf) <- c("LR","DT","NN","SVM")

rl1\$tst $\rightarrow$ tst

hms(tst\$alvo,hdf)

plotrc(rl1\$tst,hdf)

$\cdots$

\#\# PCA with counting variants

' $\{r$ pca tabela, echo=FALSE, message=FALSE, warning=FALSE $\}$

pcaf(final, "Principal Component Analysis")

\#\# PCA with raw vcf

' $\{$ r pca dados brutos, echo=FALSE, message=FALSE, warning=FALSE $\}$ snpgdsVCF2GDS(vcf.fn, "final.gds", method="biallelic.only")

genofile $<-$ openfn.gds("final.gds") 
out <- NULL

pca_vcf(genofile,out,dados)

' $\{$ r cluster, echo=FALSE, message $=$ FALSE, warning $=$ FALSE $\}$

filter(final, kmeans $==1$ ) $->$ final1

filter(final, kmeans $==2$ ) $->$ final2

‥

\# Cluster 1

\# \# LoF

\#\# Loss-of-function grouping

' $\{\mathrm{r}$, echo=FALSE, message $=$ FALSE, warning $=$ FALSE $\}$

hp_lof(final1,2)

\#\# \# Loss-of-function ROC curve

' $\{\mathrm{r}$, echo=FALSE, message $=$ FALSE, warning $=$ FALSE $\}$

lof_roc(final1)

\#\# Logistic Regression

' $\{r$, echo=FALSE, message $=$ FALSE, warning $=$ FALSE $\}$

$\mathrm{rl}($ final1, .5,.5) $->\mathrm{rl} 11$

$\mathrm{kbl}(\mathrm{rl11} \$ \mathrm{t} 2)$

\# \# Decicion Tree

' $\{\mathrm{r}$, echo=FALSE, message $=$ FALSE, warning=FALSE $\}$ tree(final1,.5) -> afinal1

treetest(final1, afinal1[[3]],.5) -> tr1

...

\#\# Neural Network

' $\{\mathrm{r}$, echo $=$ FALSE, message $=$ FALSE, warning $=$ FALSE $\}$ rede(final1,.5) -> nn1 
\#\# Support Vector Machine

' $\{r$, echo=FALSE, message $=$ FALSE, warning=FALSE $\}$

spvm(final1,.5,.1) -> sp11

sp11\$p1

\#\# Classification Performance

' $\{r$, echo=FALSE, message $=$ FALSE, warning=FALSE $\}$

hdf $<-$

data.frame $(\mathrm{LR}=\mathrm{rl11} \$ \mathrm{tst}[$ 'pred'],DT=tr1, $\mathrm{NN}=\mathrm{nn} 1, \mathrm{SVM}=\mathrm{sp} 11 \$ \mathrm{tst}[$ 'pred'])

colnames(hdf) <- c("LR","DT","NN","SVM")

rl11\$tst $->$ tst

hms(tst\$alvo,hdf)

plotrc(rl11\$tst,hdf)

\#\# Principal Component Analysis

' $\{r$, echo=FALSE, message $=$ FALSE, warning=FALSE $\}$

pcaf(final1, "Principal Component Analysis")

\# Cluster 2

\#\# LoF

\#\#\# Loss-of-function grouping

' $\{r$ r, echo=FALSE, message $=$ FALSE, warning=FALSE $\}$

hp_lof(final2,2)

\#\#\# Loss-of-function ROC curve

' $\{r$, echo=FALSE, message $=$ FALSE, warning=FALSE $\}$

lof_roc(final2)

\#\# Logistic Regression 
' $\{r$, echo=FALSE, message $=$ FALSE, warning $=$ FALSE $\}$

rl(final2,.5,.5) -> rl12

$\mathrm{kbl}(\mathrm{rl} 12 \$ \mathrm{t} 2)$

\#\# Decicion Tree

' $\{r$, echo=FALSE, message=FALSE, warning=FALSE $\}$ tree(final2,.5) -> afinal2

treetest(final2,afinal2[[3]],.5) -> tr2

‥

\#\# Neural Network

' $\{r$ r, echo=FALSE, message $=$ FALSE, warning=FALSE $\}$

rede(final2,.5) $\rightarrow>$ nn2

‥

\#\# Support Vector Machine

' $\{r$, echo=FALSE, message $=$ FALSE, warning=FALSE $\}$

spvm(final2,.5,.1) -> sp12

sp12\$p1

\#\# Classification Performance

' $\{r$, echo=FALSE, message $=$ FALSE, warning=FALSE $\}$

hdf $<-$

data.frame (LR=rl12\$tst['pred'],DT=tr2,NN=nn2,SVM=sp12\$tst['pred'])

colnames(hdf) <- c("LR","DT","NN","SVM")

rl12\$tst $\rightarrow$ tst

hms(tst\$alvo,hdf)

plotrc(rl12\$tst,hdf)

\#\# Principal Componente Analysis

' $\{r$, echo=FALSE, message $=$ FALSE, warning=FALSE $\}$

pcaf(final2, "Principal Component Analysis")

$\cdots$

\# Only Exonic Regions 


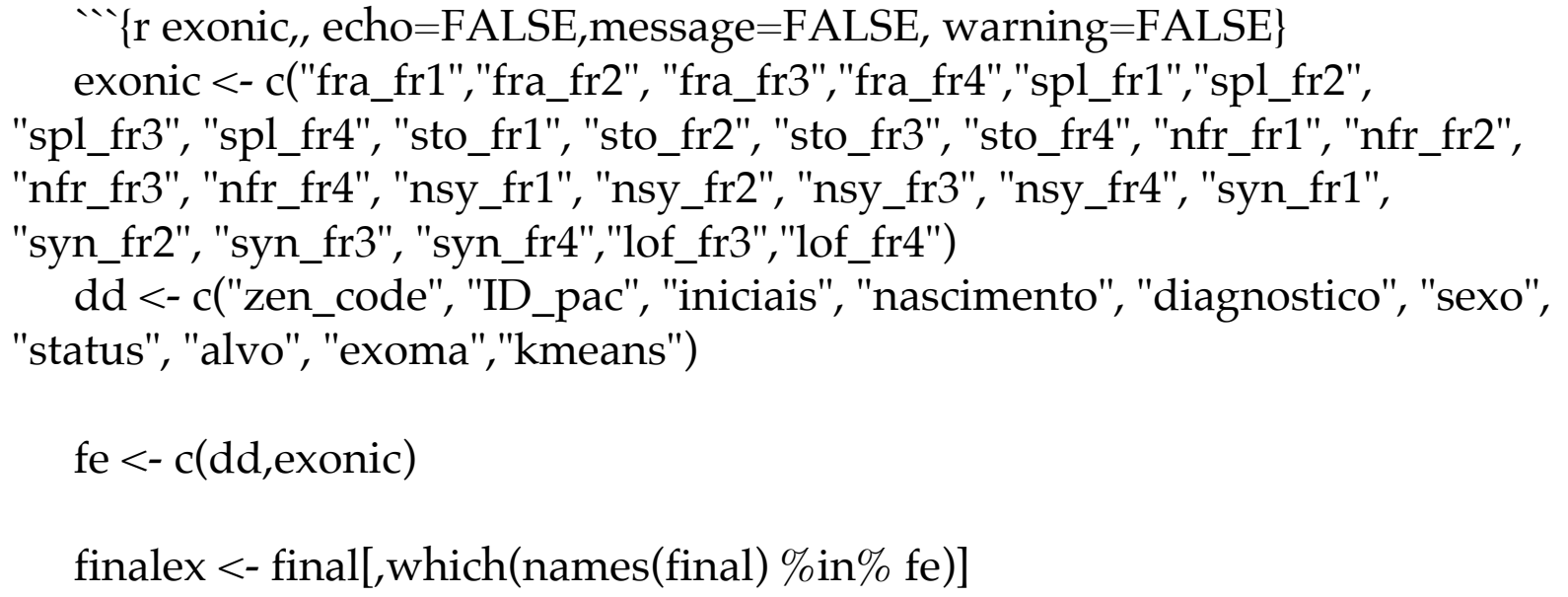


' $\{r$, echo=FALSE, message $=$ FALSE, warning $=$ FALSE $\}$

rede(finalex,.5) $\rightarrow>$ nnex

‥

\#\# Support Vector Machine

' $\{r$, echo=FALSE, message $=$ FALSE, warning=FALSE $\}$

$\operatorname{spvm}($ finalex, $.5, .1)->$ spex

spex $\$ p 1$

\#\# Classification Performance

$\cdots\{r$, echo=FALSE, message $=$ FALSE, warning=FALSE $\}$

hdf $<-$

data.frame $(\mathrm{LR}=$ rlex $\$$ tst['pred'],DT=trex, $\mathrm{NN}=$ nnex,SVM=spex $\$$ tst['pred'])

colnames(hdf) <- c("LR","DT","NN","SVM")

rlex\$tst $\rightarrow$ tst

hms(tst\$alvo,hdf)

plotrc(rlex\$tst,hdf)

\#\# PCA with exonic variants

' $\{r$, echo=FALSE, message $=$ FALSE, warning=FALSE $\}$

pcaf(finalex, "Principal Component Analysis")

\# Only Regulatory Regions

'“ $\{r$ regulatory, echo=FALSE, message $=$ FALSE, warning=FALSE $\}$

finalrg <- final[,-which(names(final) \%in\% exonic)]

finalrg\$lof_fr3 $<-$

finalrg\$ncRNA_fr3+finalrg\$miRNA_fr3+finalrg\$bnd_fr3+finalrg\$reg_fr3 finalrg\$lof_fr4 <-

finalrg\$ncRNA_fr4+finalrg\$miRNA_fr4+finalrg\$bnd_fr4+finalrg\$reg_fr4

\#\# All regulatory Regions

\#\#\# All regulatory grouping

$\cdots\{r$, echo=FALSE, message $=$ FALSE, warning=FALSE $\}$ 
hp_lof(finalrg,2)

\#\#\# All regulatory ROC curve

' $\{r$, echo=FALSE, message $=$ FALSE, warning=FALSE $\}$

lof_roc(finalrg)

\#\# Logistic Regression

' $\{r$, echo=FALSE, message $=$ FALSE, warning=FALSE $\}$

rl(finalrg, .5,.5) -> rlrg

kbl(rlrg\$t2)

\#\# Decicion Tree

' $\{r$, echo=FALSE, message $=$ FALSE, warning $=$ FALSE $\}$

tree(finalrg,.5) -> afinalrg

treetest(finalrg, afinalrg[[3]],.5) -> trrg

\#\# Neural Network

' $\{r$, echo=FALSE, message $=$ FALSE, warning=FALSE $\}$

rede(finalrg,.5) -> nnrg

‥

\#\# Support Vector Machine

' $\{r$, echo=FALSE, message $=$ FALSE, warning=FALSE $\}$

spvm(finalrg,.5,.1) -> sprg

$\operatorname{sprg} \$ \mathrm{p} 1$

\#\# Classification Performance

' $\{r$, echo=FALSE, message $=$ FALSE, warning=FALSE $\}$

hdf $<-$

data.frame(LR=rlrg\$tst['pred'],DT=trrg,NN=nnrg,SVM=sprg\$tst['pred']) colnames(hdf) <- c("LR","DT","NN","SVM")

rlrg\$tst $\rightarrow$ tst

hms(tst\$alvo,hdf) 
plotrc(rlrg\$tst,hdf)

\#\# PCA with regulatory variants

' $\{r$, echo=FALSE, message=FALSE, warning=FALSE $\}$

pcaf(finalrg, "Principal Component Analysis")

\subsection{Attachment 25}

Table for the variants abbreviations

\begin{tabular}{|l|l|}
\hline Type of variants & \\
\hline fra & Frameshift \\
\hline sto & Stopgain/loss \\
\hline spl & Canonical splicing $(+1,+2,-1,-2)$ \\
\hline nfr & Nonframeshift \\
\hline nsy & Nonsynonymous \\
\hline syn & Synonymous \\
\hline utr3 & $3^{\prime}$-UTR \\
\hline utr5 & $5^{\prime}$-UTR \\
\hline ncRNA & Noncoding RNA \\
\hline miRNA & Micro RNA \\
\hline bnd & Transcription binding sites \\
\hline reg & Regulatory sites \\
\hline lof & Frameshift+Splicing+Stopgain/1 \\
\hline Frequencies & oss \\
\hline fr1 & \\
\hline fr2 & Above 0.05 \\
\hline fr3 & Between 0.05 and 0.01 \\
\hline fr4 & Below 0.01 \\
\hline & Singletons \\
\hline
\end{tabular}


